Supplement of Drink. Water Eng. Sci., 14, 53-71, 2021

https://doi.org/10.5194/dwes-14-53-2021-supplement

(C) Author(s) 2021. This work is distributed under

the Creative Commons Attribution 4.0 License.

(c) (i)

Supplement of

\title{
Can terminal settling velocity and drag of natural particles in water ever be predicted accurately?
}

Onno J. I. Kramer et al.

Correspondence to: Onno J. I. Kramer (onno.kramer@waternet.nl)

The copyright of individual parts of the supplement might differ from the CC BY 4.0 License. 


\section{Contents}

S1 Materials and methods

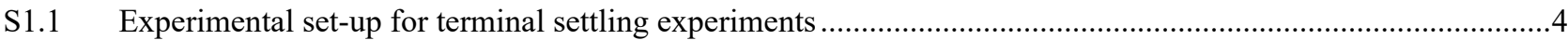

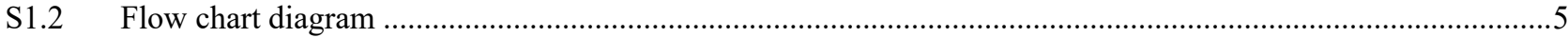

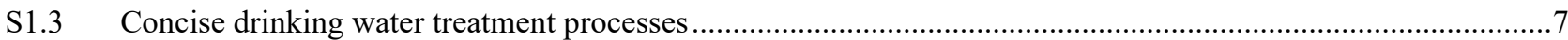

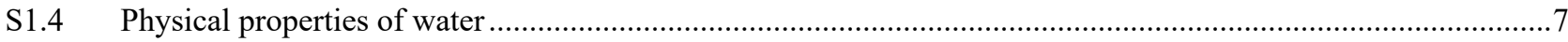

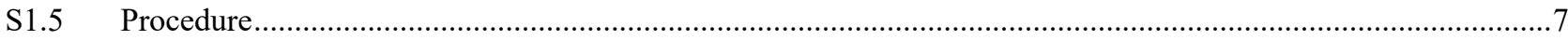

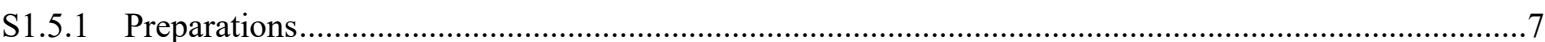

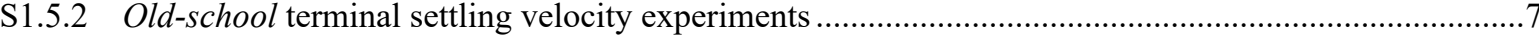

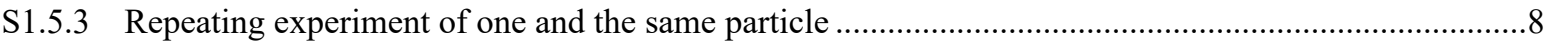

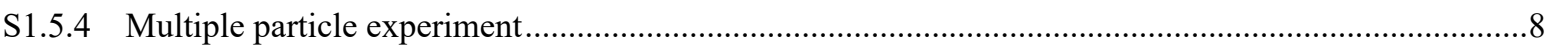

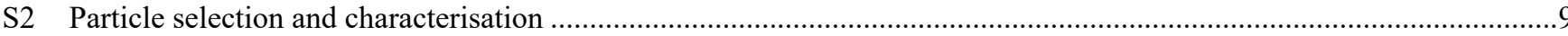

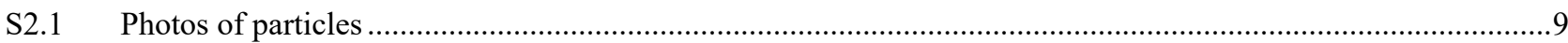

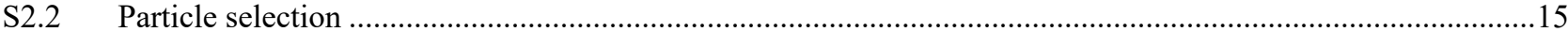

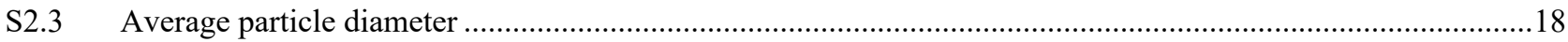

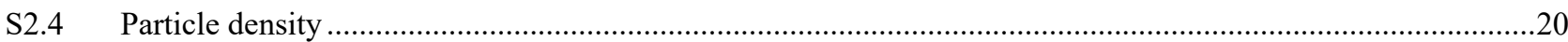

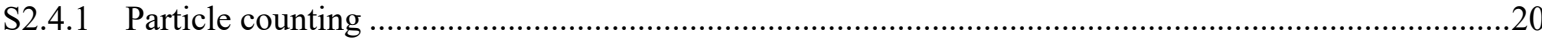

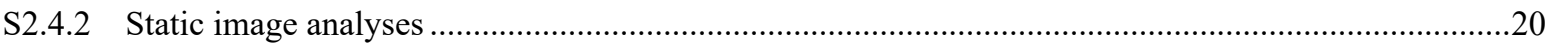

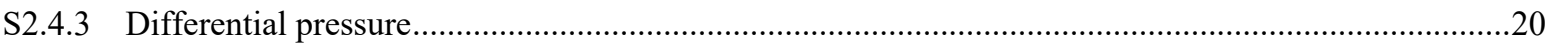

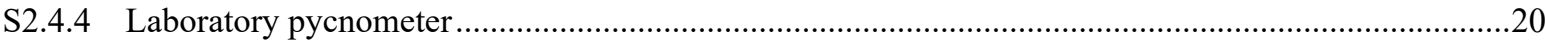

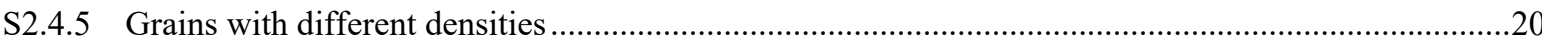

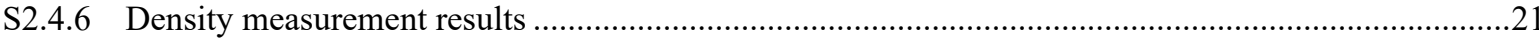

S2.5 Particle characterisation and morphological properties ....................................................................21

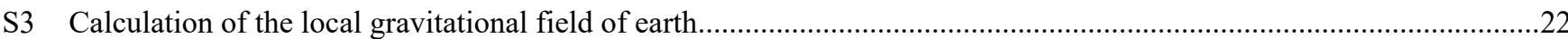

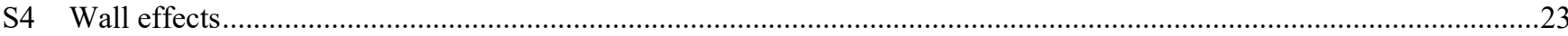

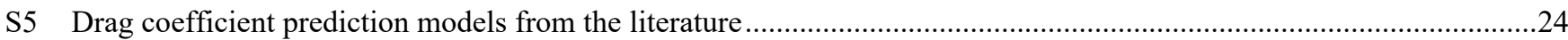

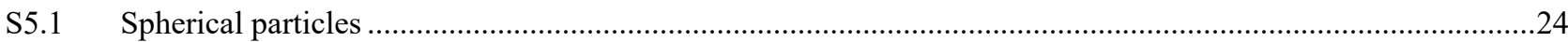

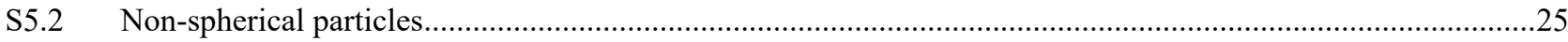

S5.3 More complex prediction models using more morphological properties ....................................................26

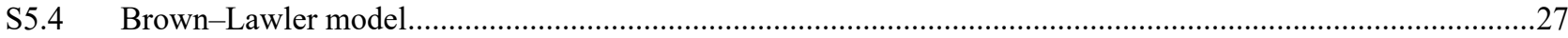

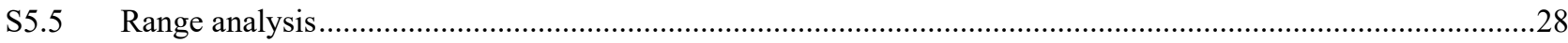

S5.6 Standard drag curve with average values and prediction models ............................................................29

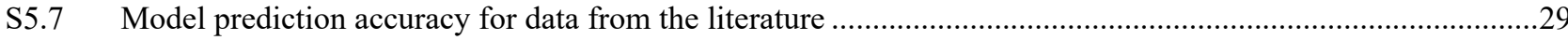


S6 Uncertainty analysis

S6.1 Basic equations

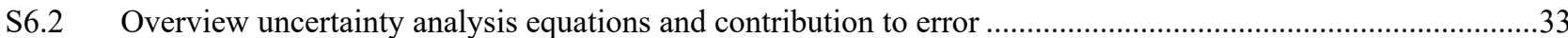

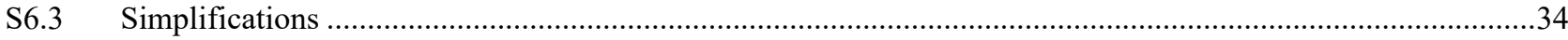

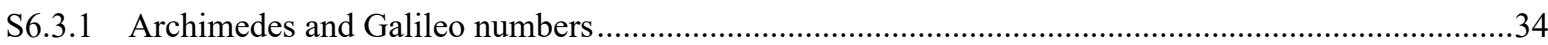

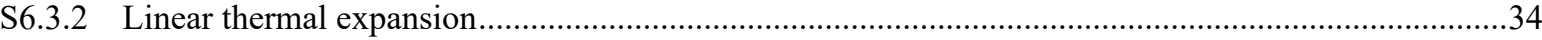

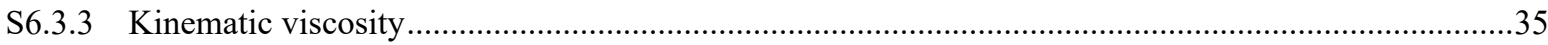

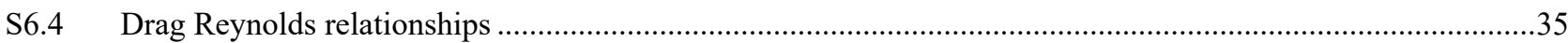

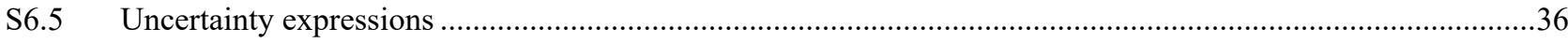

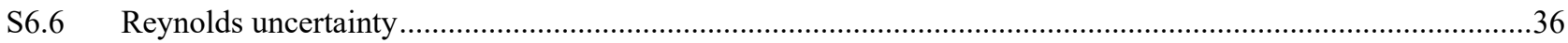

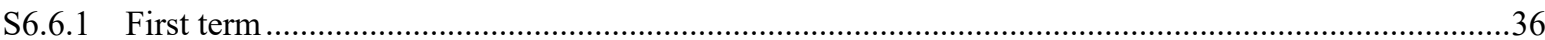

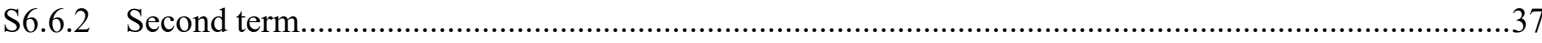

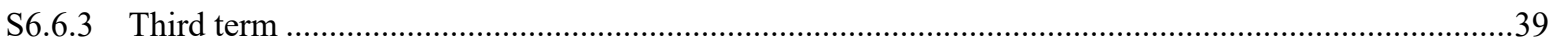

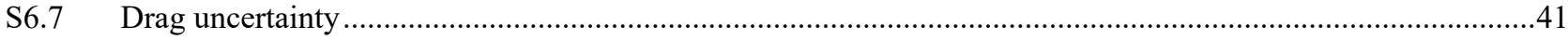

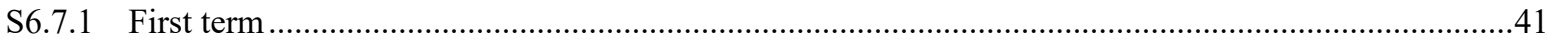

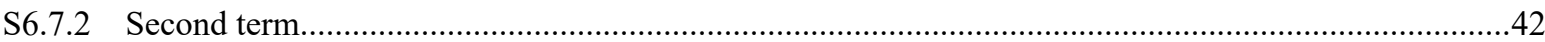

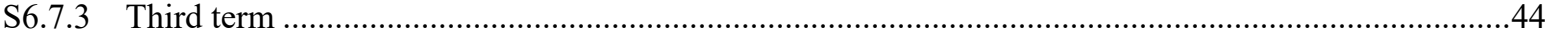

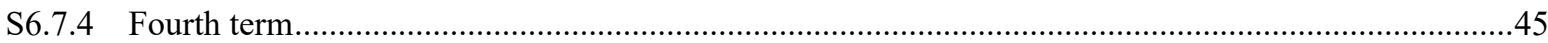

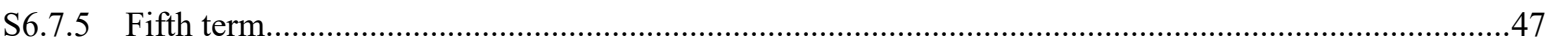

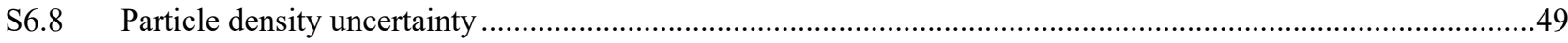

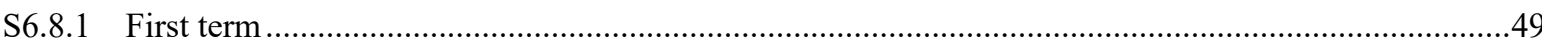

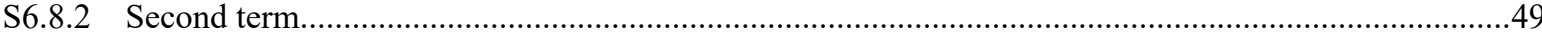

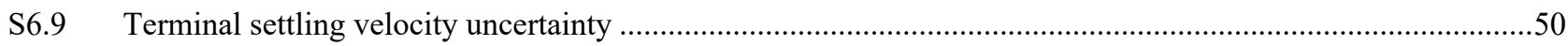

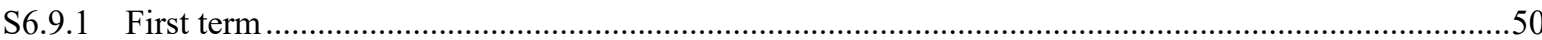

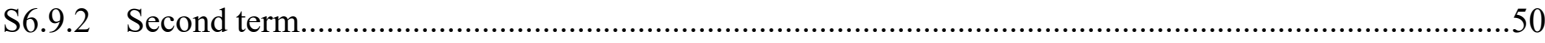

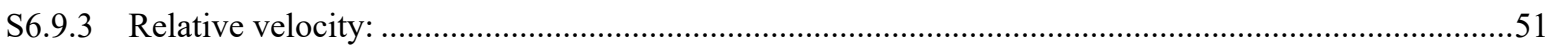

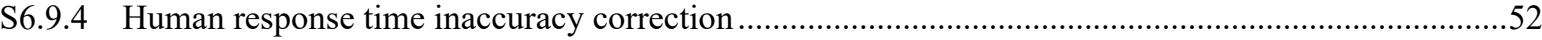

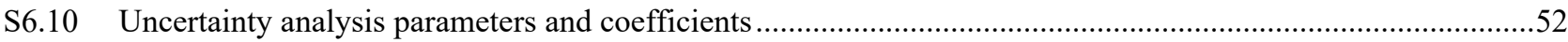

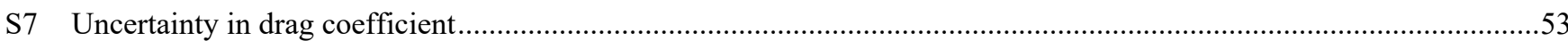

S7.1 Natural and processed highly non-spherical polydisperse particles ......................................................53

S7.2 Medium non-spherical polydisperse particles applied in water softening ...............................................53

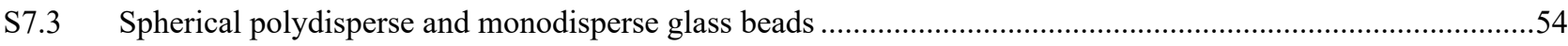

S7.4 Repetitive experiment with highly spherical monodisperse single glass beads .........................................55 
S7.5 Highly spherical monodisperse glass beads and wall effects .....................................................................56

S7.6 Highly spherical monodisperse glass beads in different columns ...................................................................56

S7.7 Highly spherical monodisperse glass beads with different fall lengths .............................................................57

S7.8 Highly spherical polydisperse synthetic particles ..................................................................................57

S7.9 Highly spherical monodisperse metal balls ………................................................................................5

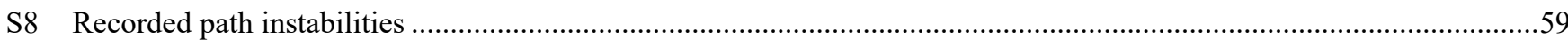

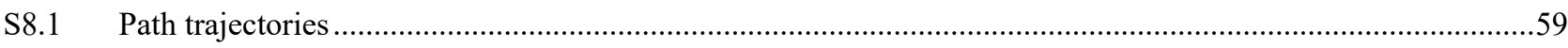

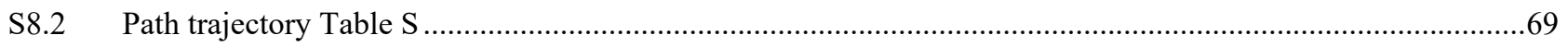

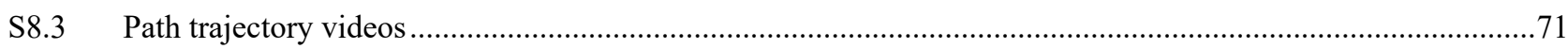

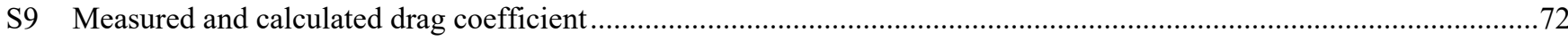

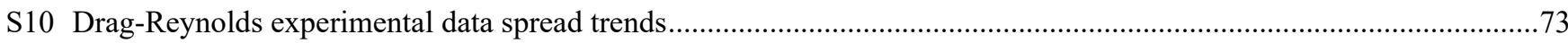

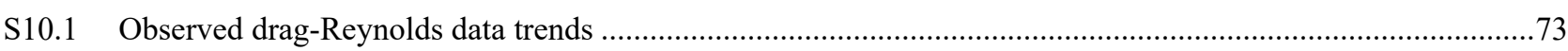

S10.2 Error made by the laboratory researcher.....................................................................................................

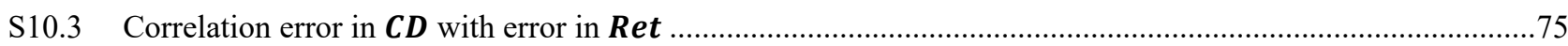

S10.4 Direct simplified dependency of the deviation to the variation in particle diameter.........................................76

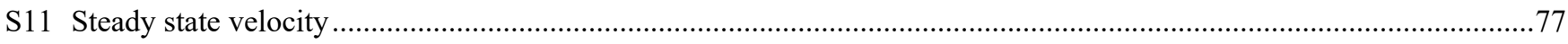

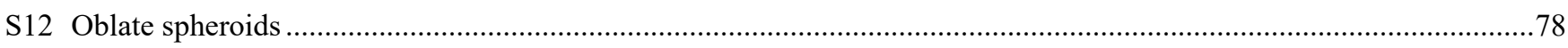

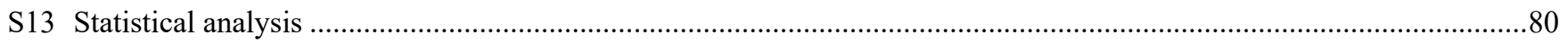

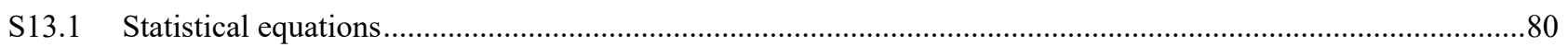

S13.2 Propagated effect of parameter uncertainties on the drag coefficient and terminal Reynolds number...............81

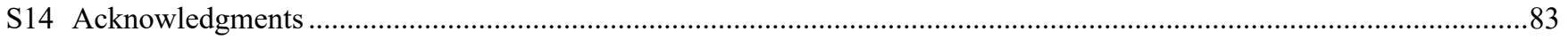

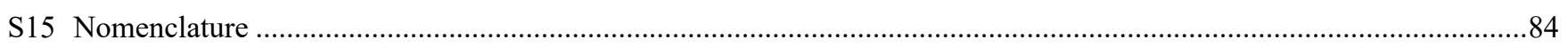

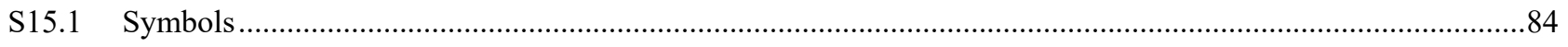

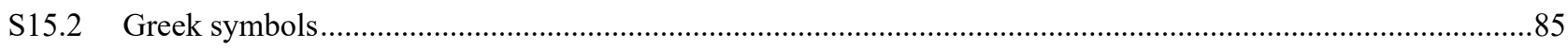

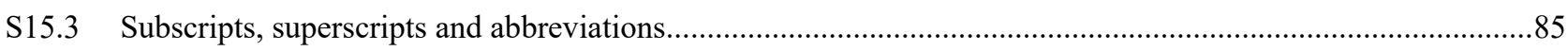

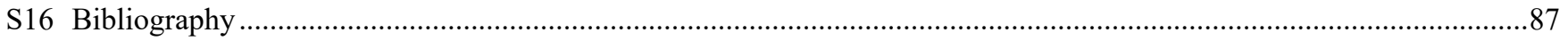

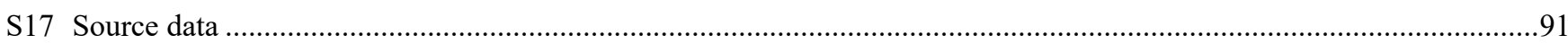

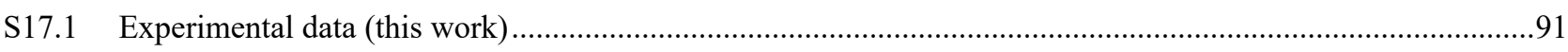

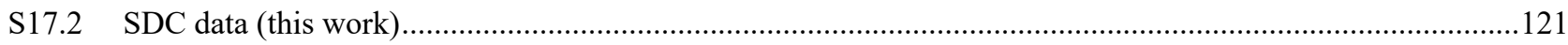

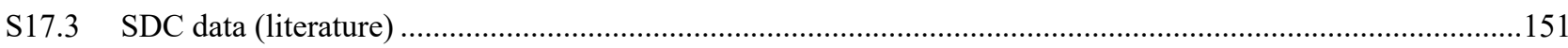




\section{S1 Materials and methods}

\section{S1.1 Experimental set-up for terminal settling experiments}

Experimental columns $(D=57 \mathrm{~mm})$ were designed at Waternet for liquid-solid fluidisation (Kramer et al., 2020b) terminal settling experiments and installed at three locations: in Waternet's Weesperkarspel drinking water pilot plant located in Amsterdam, the Netherlands, at the University of Applied Sciences Utrecht, the Netherlands, and at Queen Mary University of London, United Kingdom (Figure S1 and Figure S2). A schematic overview can be found in Figure S4. Extra experimental columns $(\mathrm{D}=125 \mathrm{~mm})$ were installed at Waternet and at the University of Applied Sciences Utrecht (Figure $\mathrm{S} 3$ ). In addition, an advanced experimental pilot set-up at TU Delft was used to determine particle path trajectories $3 \mathrm{D}$ in a quiescent fluid.

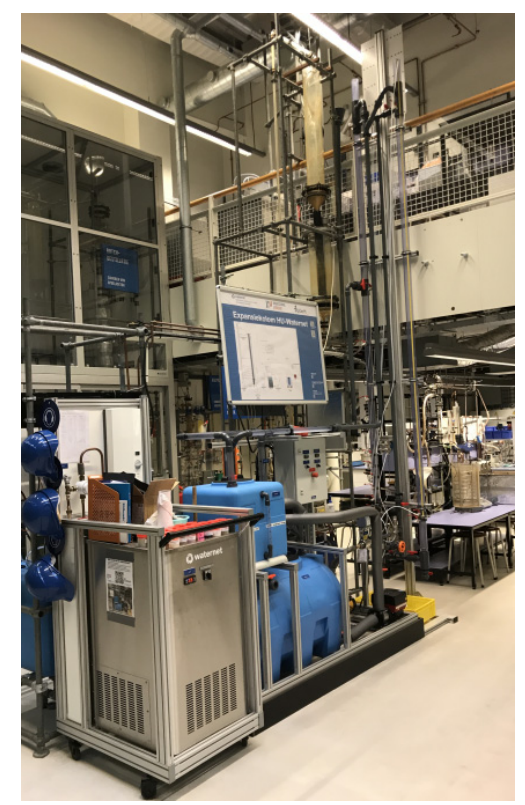

Figure S1 Experimental pilot set-up in Amsterdam, Utrecht and London with temperature control

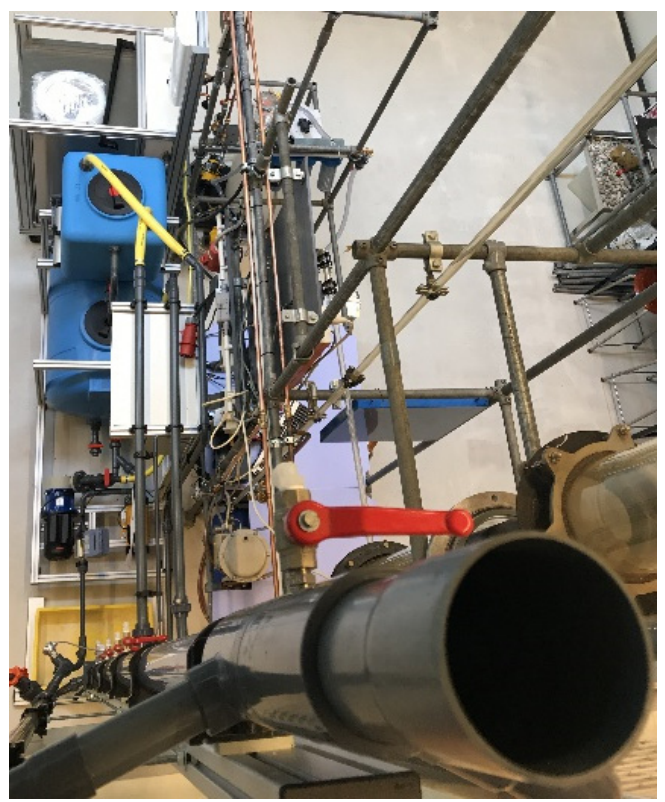

Figure S2 Column top view $D=57 \mathrm{~mm}$

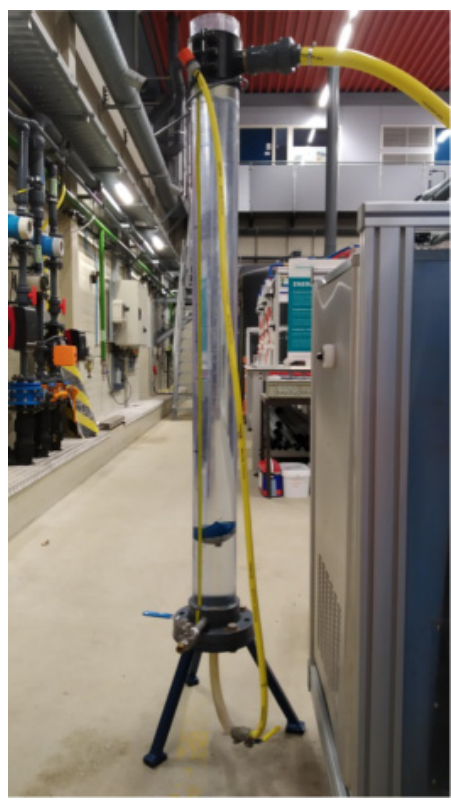

Figure S3 Terminal settling column $D=125$ $\mathrm{mm}$ 
S1.2 Flow chart diagram

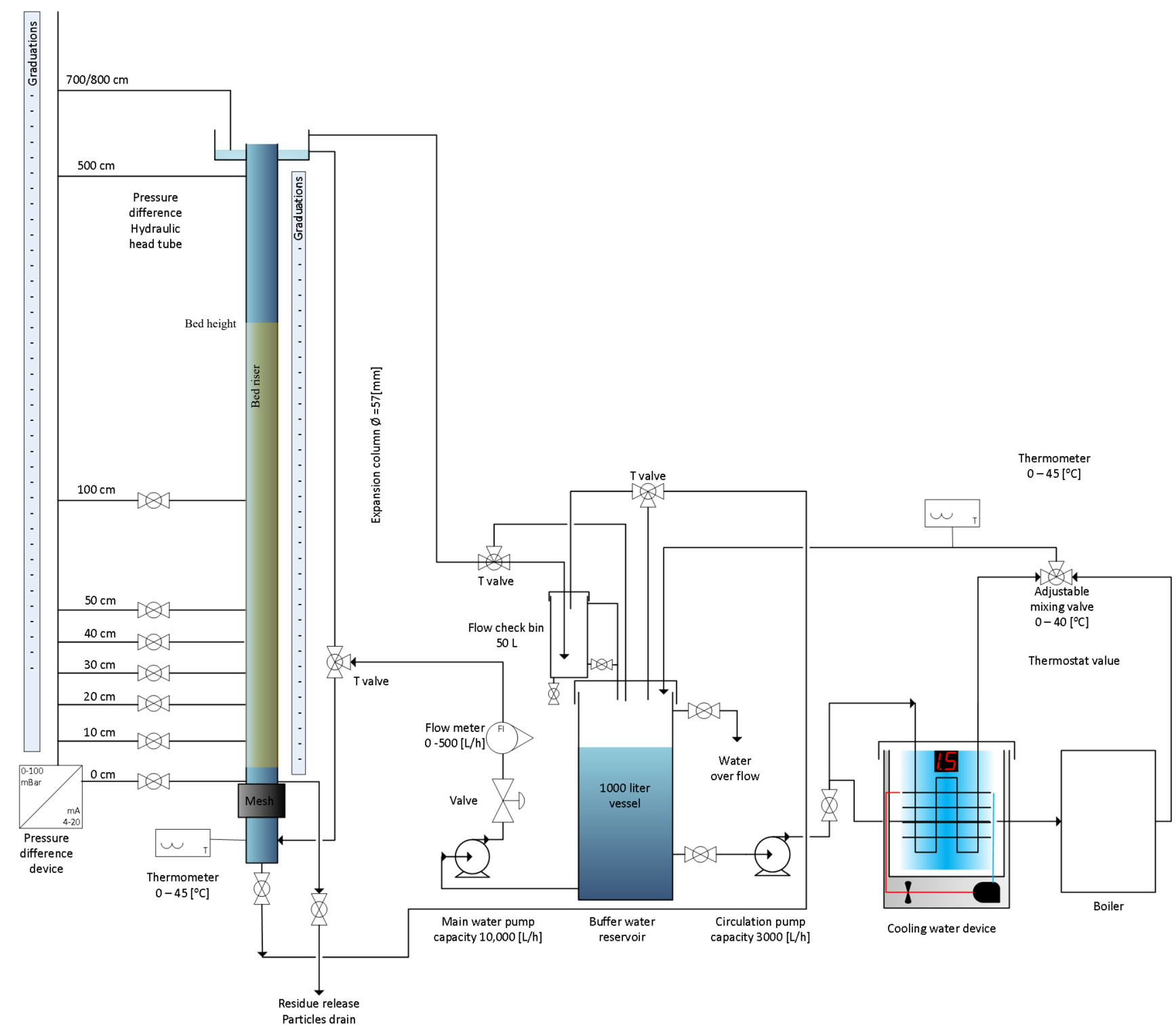

Figure S4 Experimental set-up 


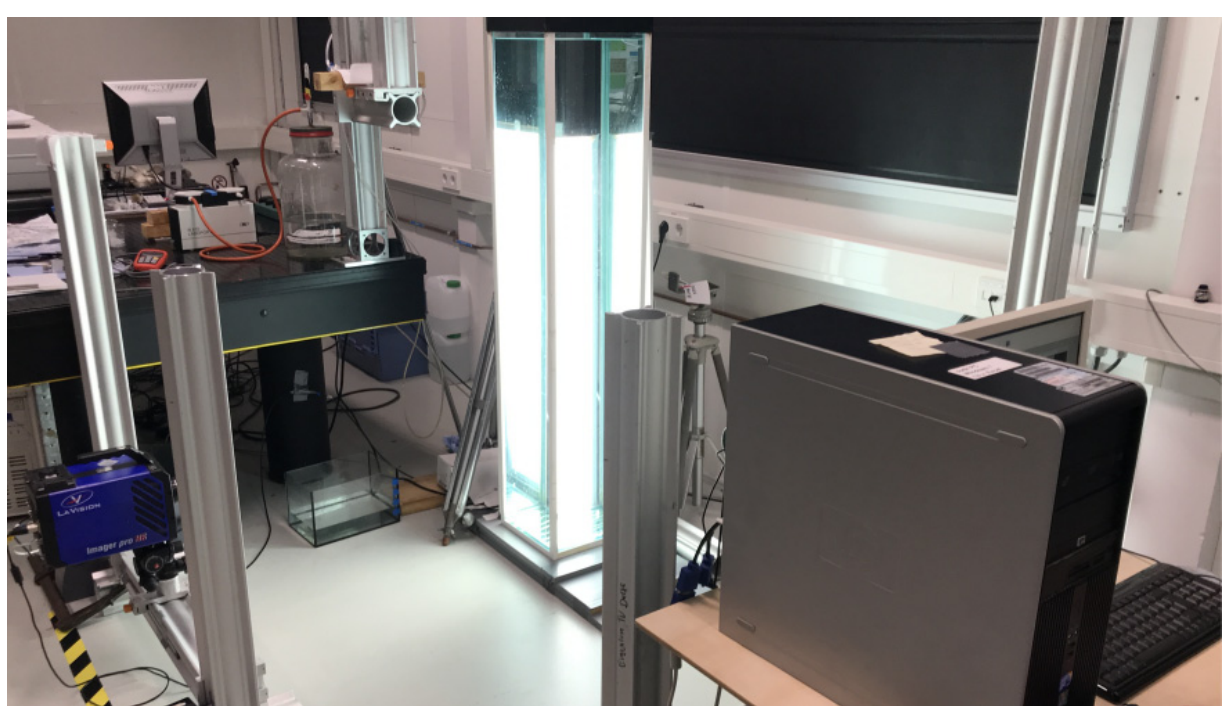

Figure S5 Experimental pilot set-up at TU Delft D $=300 \mathrm{~mm}$ to determine particle path trajectories $3 D$ in a quiescent fluid

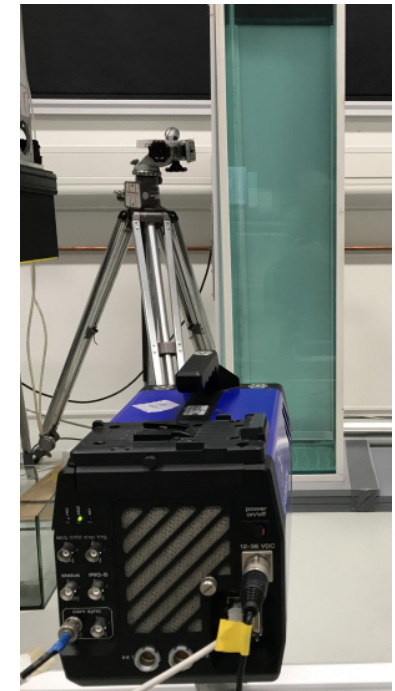

Figure S6 High-speed camera

The design of the experiment involved tracking the 3D position of a sphere falling in a tank containing still water, using 3D particle tracking velocimetry, to accurately capture the path instabilities. Installed components included a temperature conditioned tank, controlled high-speed cameras, data acquisition, particle release mechanism, LEDs and a water bath for pre-wetting purposes.

The sphere is able to travel several hundred sphere diameters (Jenny et al., 2004) before it reaches the field of view of the cameras. It is possible to track the sphere for sufficiently large distance $\left(70 d_{p}\right.$ for the largest sphere in the current study and $375 d_{p}$ for the smallest one).The advanced experimental set-up is thoroughly explained and discussed in (Raaghav, 2019). 


\section{S1.3 Concise drinking water treatment processes}

The raw water originates from seepage water from the Bethune polder. This water is pre-treated by coagulation and sedimentation, followed by approximately 90 days' retention in a lake reservoir. Subsequently, it is filtered through rapid sand filters. The treatment plant that follows contains ozonation, pellet-softening, biological activated carbon filtration and slow sand filtration. Chlorination is not needed.

\section{S1.4 Physical properties of water}

The density of the water was retrieved from the Handbook of Chemistry and Physics by (Rumble, 2019) and Perry's Chemical Engineers' Handbook (Perry and Green, 2007). The dynamic viscosity is given by the Vogel-Fulcher-Tammann equation (Vogel, 1921) Equation (S1), and improved by (Civan, 2007) as follows:

$$
\eta=\frac{1}{c_{5}} e^{\left(c_{1}+\frac{c_{2}}{c_{3}+\left(T+c_{4}\right)}\right)}
$$

\begin{tabular}{ll} 
Table S1 & $\begin{array}{l}\text { Vogel-Fulcher-Tammann equation parameters } \\
273<T[K]<373\end{array}$ \\
\cline { 1 - 2 } Parameter & Value \\
\hline C $1_{1}$ & -3.7188 \\
C $_{2}$ & 578.919 \\
C $_{3}$ & -137.546 \\
C $_{4}$ & 273 \\
C $_{5}$ & 1000 \\
\hline
\end{tabular}

\section{S1.5 Procedure}

\section{S1.5.1 Preparations}

In the experiments, the produced drinking water was used (§S1.4). The set-up (Figure S4) consisted of a 4-meter transparent PVC pipe with an inner diameter of $57 \mathrm{~mm}$. Water temperature was regulated with a boiler, a cooler and a thermostat by recirculating water through a buffer vessel connected to a water reservoir. The effect of gradient in temperature in the vertical direction was negligible. An overflow at the top of the reactor returned water to the buffer vessel. Water from the buffer vessel was pumped through the reservoir connected to the thermostat which was set to a programmed water temperature. During the terminal settling experiments, the water pump was turned off. To avoid a large amount of disturbances in the fluid, a gap was taken of at least 15 minutes to obtain a stagnant system. To determine the terminal settling velocity in quiescent water, grains were pre-wetted to avoid air bubbles. A tweezer was used to drop individual grains at the centre of the cylindrical tubes. For the traditional experiments, the fall time was measured with a stopwatch and a measuring tape assembled to the tubes.

\section{S1.5.2 Old-school terminal settling velocity experiments}

The settling behaviour of single particles was determined for various materials and for different grain sizes. The temperature was carefully controlled by flowing water of the exact temperature through the column before each experiment and by regularly repeating this process throughout the experiment. Individual particles were dropped at the top of the column. Steady state velocities (Equations given in Chapter S11) were reached during one second and before a distance of $L=0.1 \mathrm{~m}$. 
A condition for steady state velocity is that the particle travels a distance of at least $O\left(100 \cdot d_{p}\right)$ or greater before the stop clock is switched on. After steady state velocity had been reached, the required time to elapse a defined distance $(L=0.50$ $3.75 \mathrm{~m}$ ) was measured visually by the laboratory researcher and assistant.

A log was used to register the particle and fluid properties, the dimensions of the particular experiment set-up, the recorded fall length and time as well as observations such as the fall trajectory and occurring path instabilities.

A wide range of grains was selected (Table S2, Table S3, Table S4, Table S5 and Table S6) and tested for various temperatures between 3 and $37^{\circ} \mathrm{C}$. A powerful flashlight at the top of the column supported the visual determination.

\section{S1.5.3 Repeating experiment of one and the same particle}

For one specific sequence, the terminal settling velocity of one individual $3 \mathrm{~mm}$ spherical monodisperse glass bead (type B162 surface polished dark blue SiLibeads, see Table S3) was repeated 30 times. Two iPhones were used to film the fall trajectory (first iPhone X plus) to determine the moments when the glass bead passed the start and finish marker and the time (stopwatch on second iPhone 8 plus).

\section{S1.5.4 Multiple particle experiment}

It is difficult to determine the terminal settling velocity of very small particles $\left(d_{p}<0.3 \mathrm{~mm}\right)$ such as garnet sand, owing to size and visibility issues when tracking it manually. Hindered settling is a completely different phenomenon (Richardson and Zaki, 1954); (di Felice, 1995); (Baldock et al., 2004); (Kramer et al., 2020b). Nevertheless, several attempts were made to estimate the settling velocity of small particles using the lowest possible amount multiple grains without adversely affecting the visual observation. Hence, a pinch of particles was dropped to increase the visibility of passing grains. Statistical outliers beyond $3.5 \sigma$ (or $99.95 \%$ confidence) for garnet grains were excluded from the statistical results. 


\section{S2 Particle selection and characterisation}

\section{S2.1 Photos of particles}

Photos of calcite grains were taken with a Nikon D500 camera using a Sigma $150 \mathrm{~mm} \mathrm{f/2.8} \mathrm{EX} \mathrm{DG} \mathrm{OS} \mathrm{HSM} \mathrm{lens.} \mathrm{Other}$ photos were taken with an I-phone 8 plus and a Nikon SMZ800 laboratory Stereo Microscope (480x) (Figure S28).

Morphological properties, such as the sphericity, were determined with a Retsch Camsizer XT (Retsch-Technology, 2007) (Figure S26). Accordingly, ImageJ software (Figure S27) was used for image analysis purposes (Ferreira and Rasband, 2012).

Central softening (Graveland et al., 1983) has been applied in the Netherlands since the late 1970s (Hofman et al., 2007) with garnet sand (Figure S30) being the most frequently used seeding material (van Dijk and Wilms, 1991); (van Schagen et al., 2008); (Tang et al., 2019). Based on sustainability reasons, garnet sand as a seeding material is replaced by crushed calcite (Figure S31) (Schetters et al., 2015).

Calcite pellet photos are taken from the Supplementary Material section in (Kramer et al., 2020a). Calcite pellets were extracted from full-scale pellet-softening reactors located at the Waternet facility Weesperkarspel in Amsterdam. Accordingly, the pellets were fractionated (Figure S24) using a Retsch AS 200-control sieve device (Retsch-Technology, 2007) (Figure S23) and calibrated US mesh sieves.

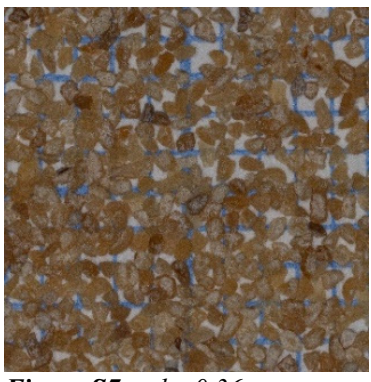

Figure $\mathbf{S 7} \quad d_{p}<0.36 \mathrm{~mm}$

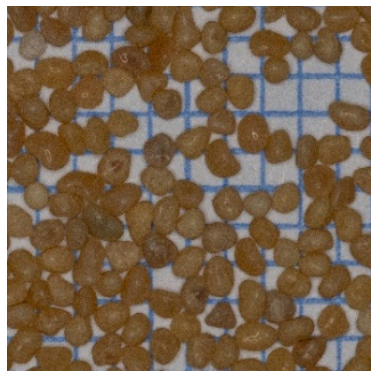

Figure $S 11 \quad 0.63<d_{p}<0.71 \mathrm{~mm}$

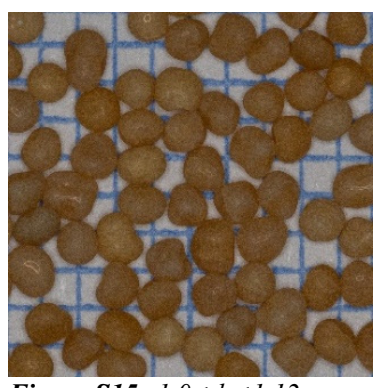

Figure $\mathbf{S 1 5} 1.0<d_{p}<1.12 \mathrm{~mm}$

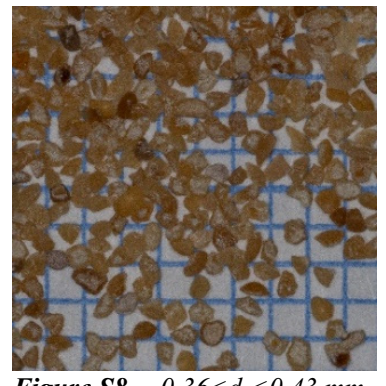

Figure S8 $\quad 0.36<d_{p}<0.43 \mathrm{~mm}$

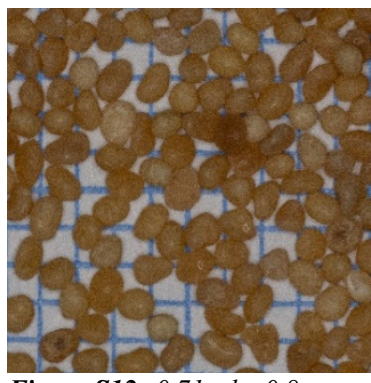

Figure S12 $0.71<d_{p}<0.8 \mathrm{~mm}$

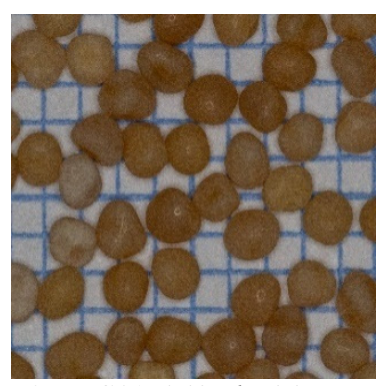

Figure S16 $1.12<d_{p}<1.25 \mathrm{~mm}$

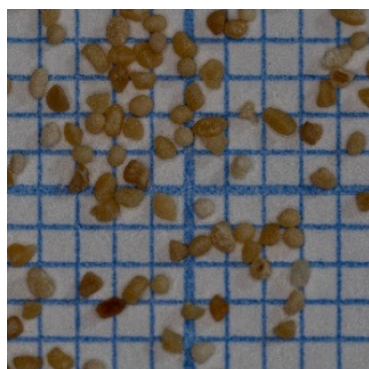

Figure S9 $0.43<d_{p}<0.5 \mathrm{~mm}$

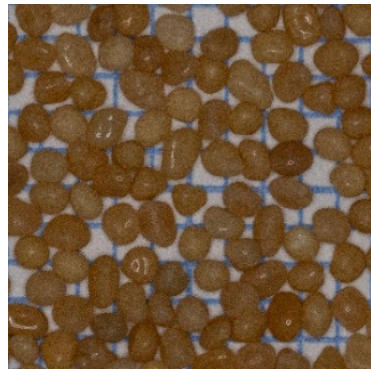

Figure S13 $0.8<d_{p}<0.9 \mathrm{~mm}$

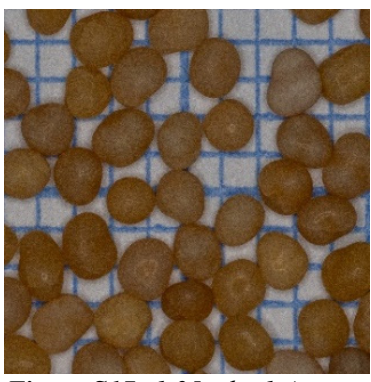

Figure $\mathbf{S 1 7} 1.25<d_{p}<1.4 \mathrm{~mm}$

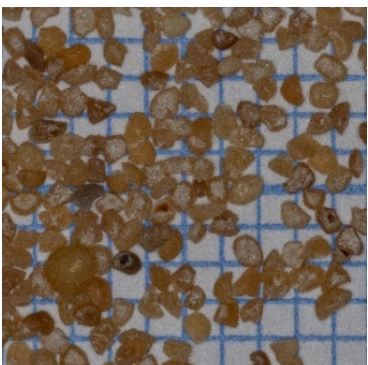

Figure S10 $0.5<d_{p}<0.63 \mathrm{~mm}$

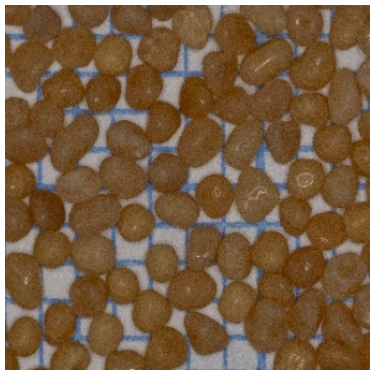

Figure S14 $0.9<d_{p}<1.0 \mathrm{~mm}$

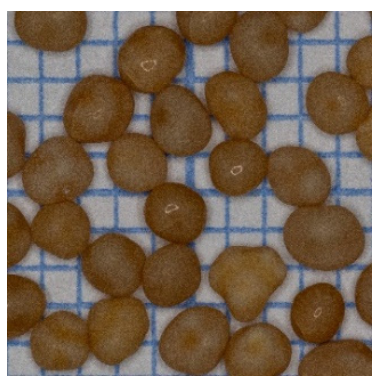

Figure $\mathbf{S 1 8} 1.4<d_{p}<1.7 \mathrm{~mm}$ 


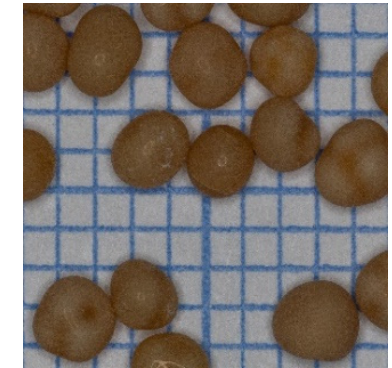

Figure S19 $1.7<d_{p}<2.0 \mathrm{~mm}$

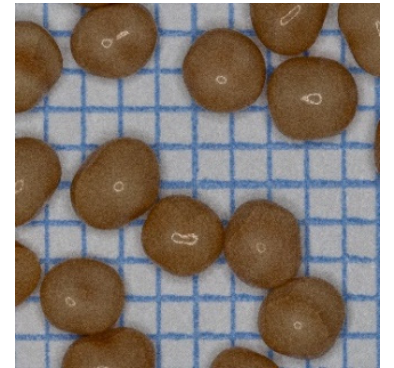

Figure S20 $2.0<d_{p}<2.4 \mathrm{~mm}$

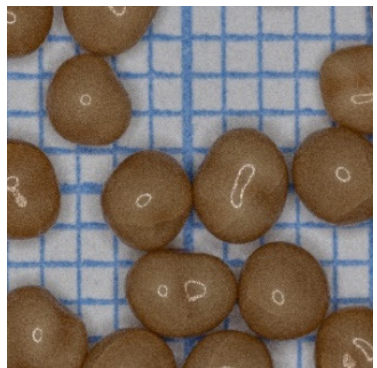

Figure S21 $2.4<d_{p}<2.8 \mathrm{~mm}$

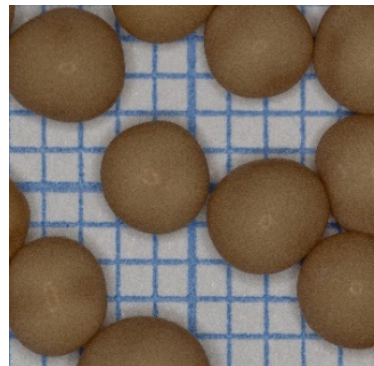

Figure $\mathbf{S 2 2} d_{p}>2.8 \mathrm{~mm}$

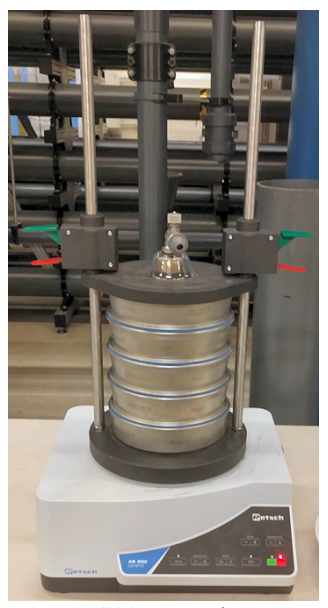

Figure S23 Retsch sieve shaker

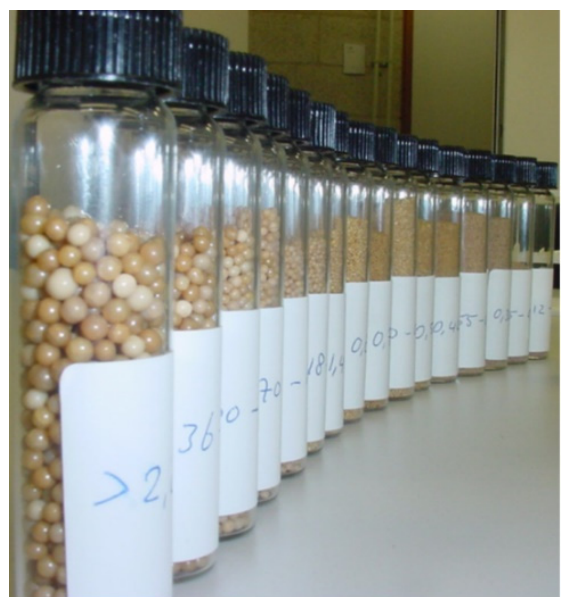

Figure S24 Calcite pellet samples in different sieve sizes

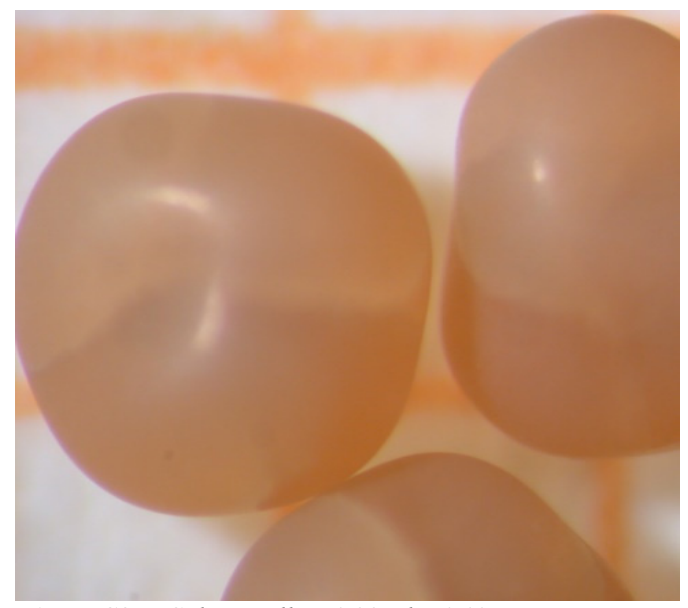

Figure S25 Calcite pellets $1.00<d_{p}<1.12 \mathrm{~mm}$

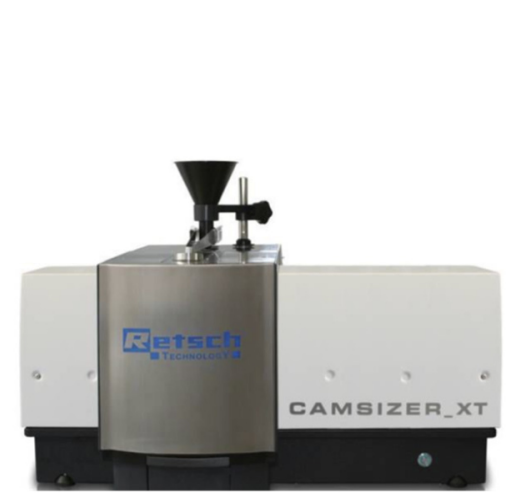

Figure S26 Retsch Camsizer XT

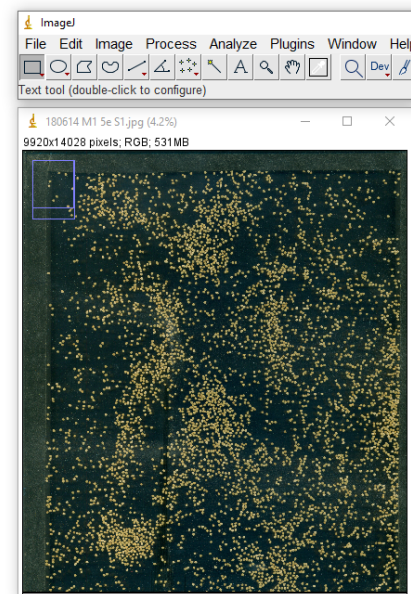

Figure S27 ImageJ version $1.52 u$

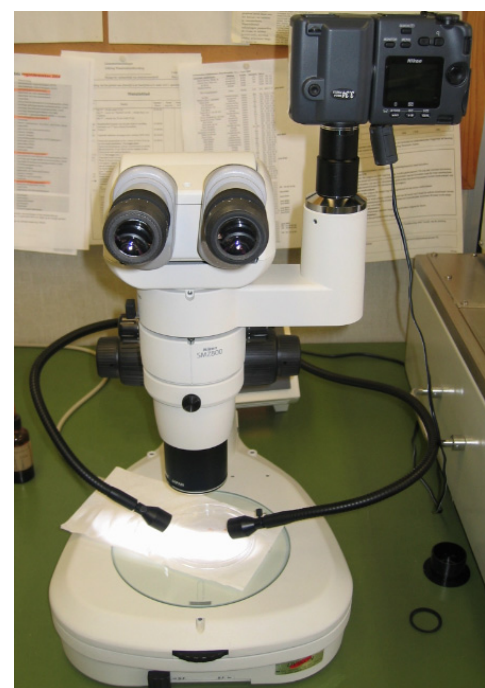

Figure S28 Stereo microscope 


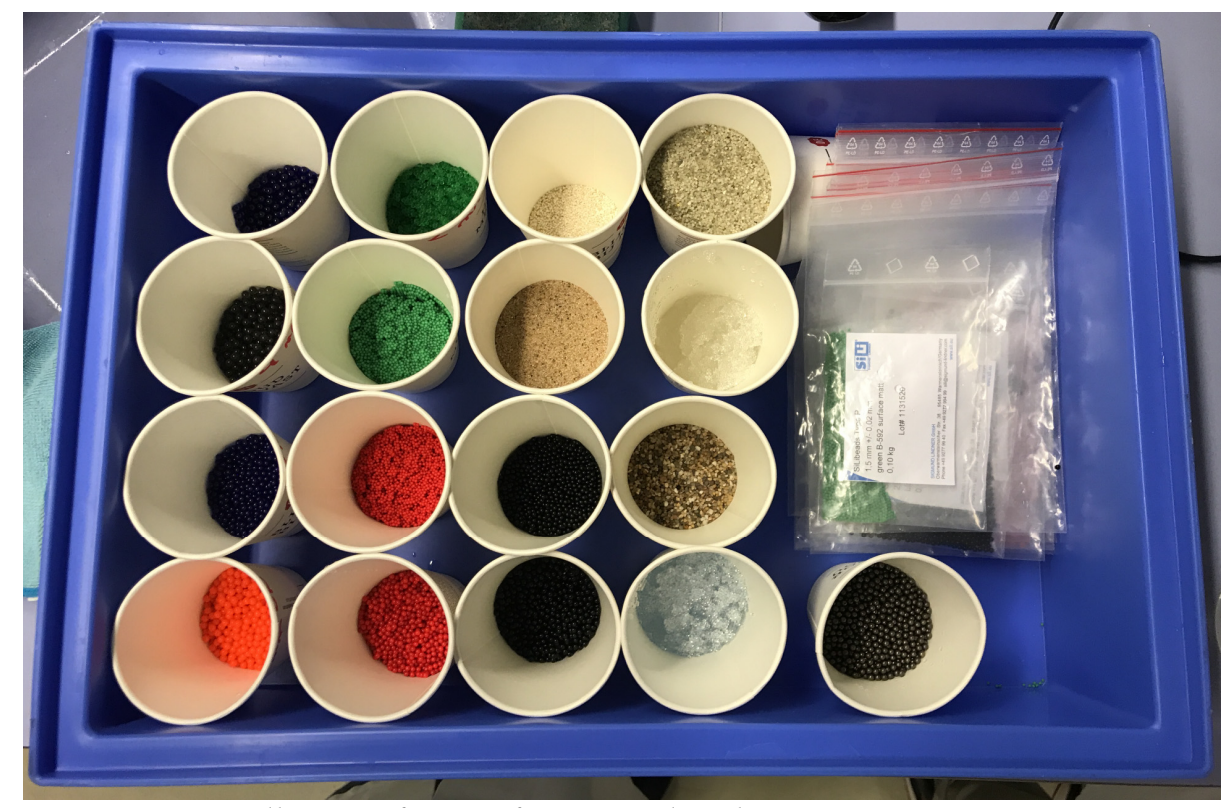

Figure S29 Collection of grains for terminal settling experiments 


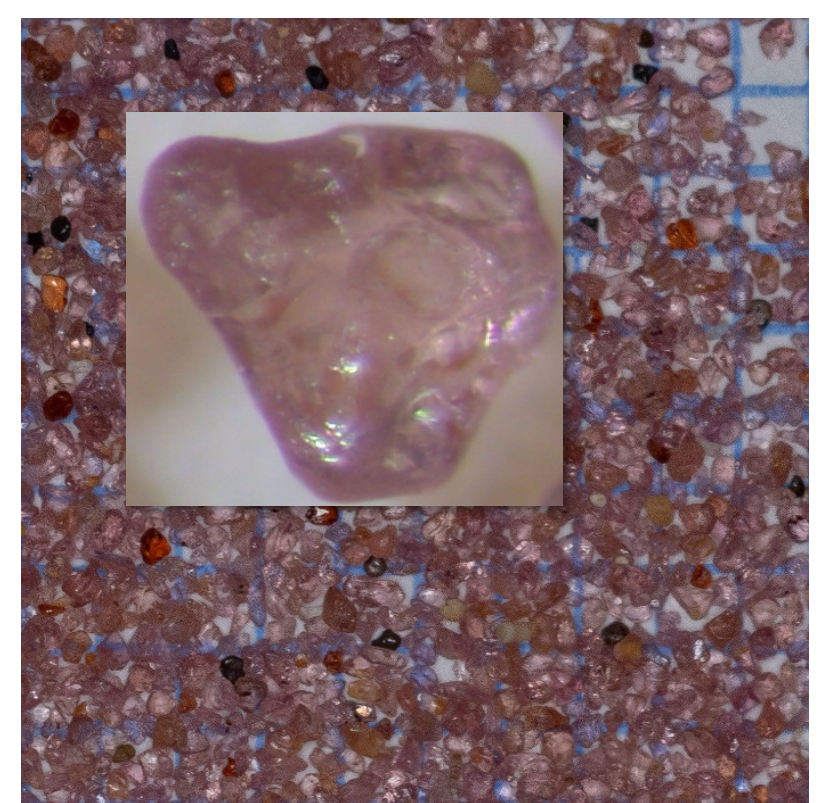

Figure S30
Garnet sand (mesh 80) $0.2<d_{p}<0.3 \mathrm{~mm}$

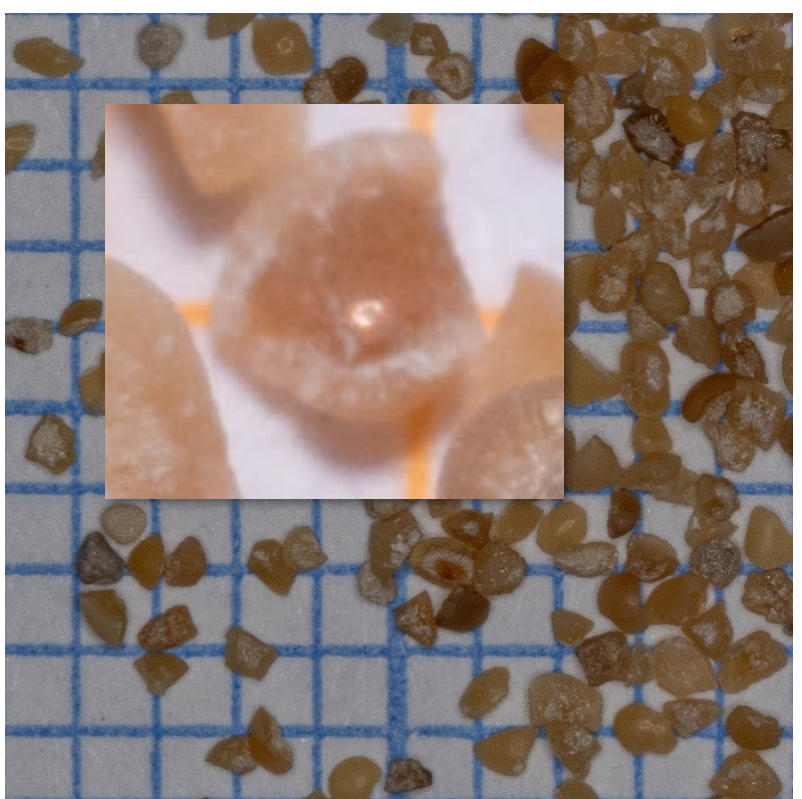

Figure S31 Crushed calcite $0.4<d_{p}<0.5 \mathrm{~mm}$ 


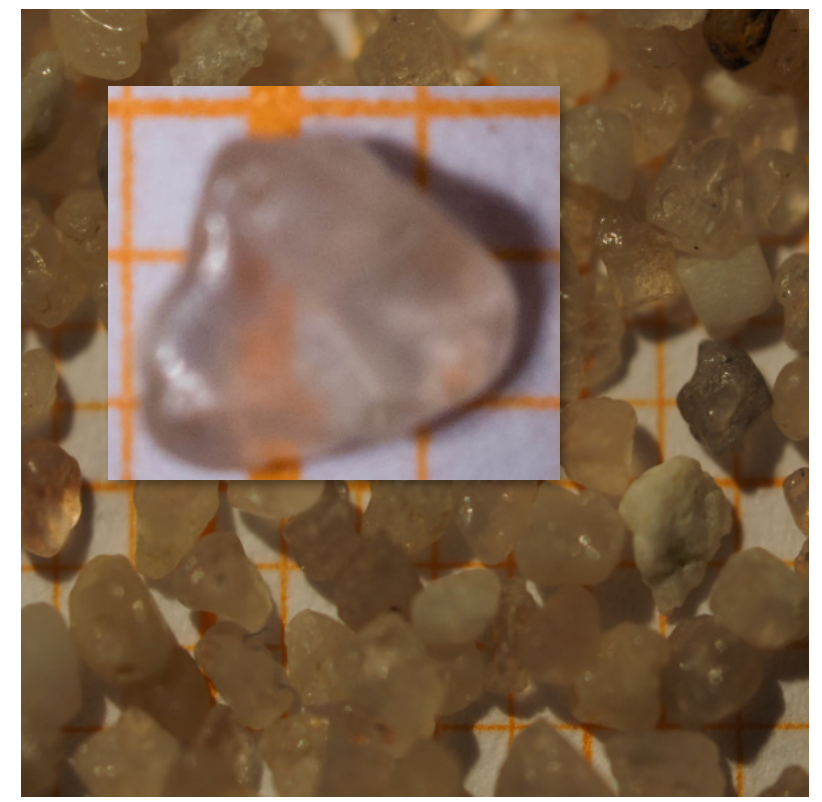

Figure S32
Rapid filter sand $0.8<d_{p}<1 \mathrm{~mm}$

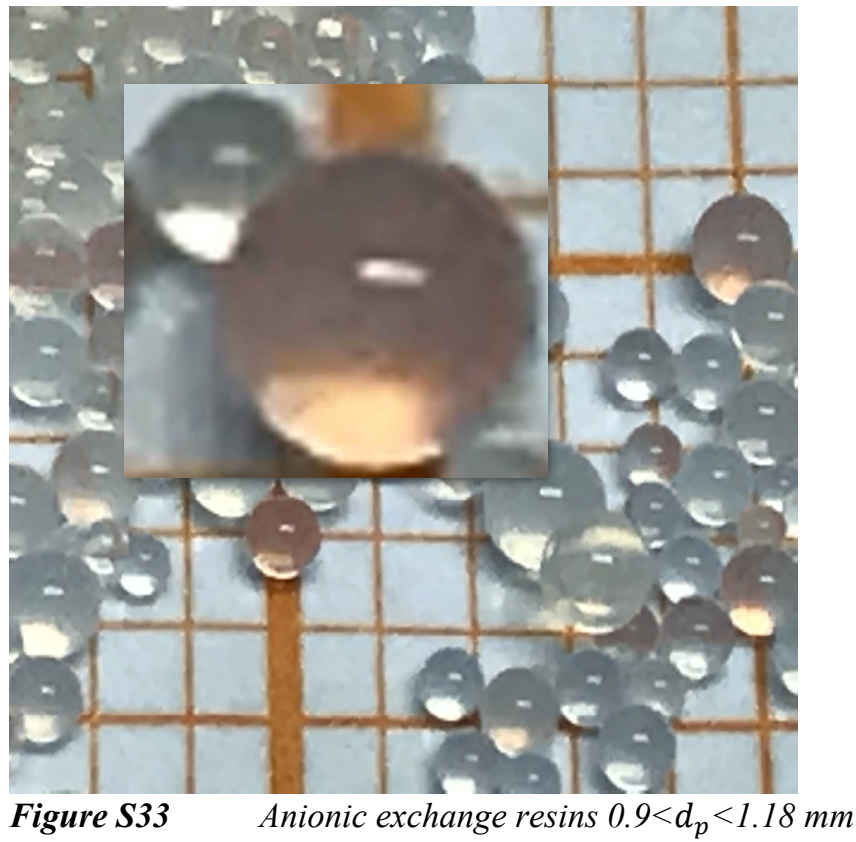




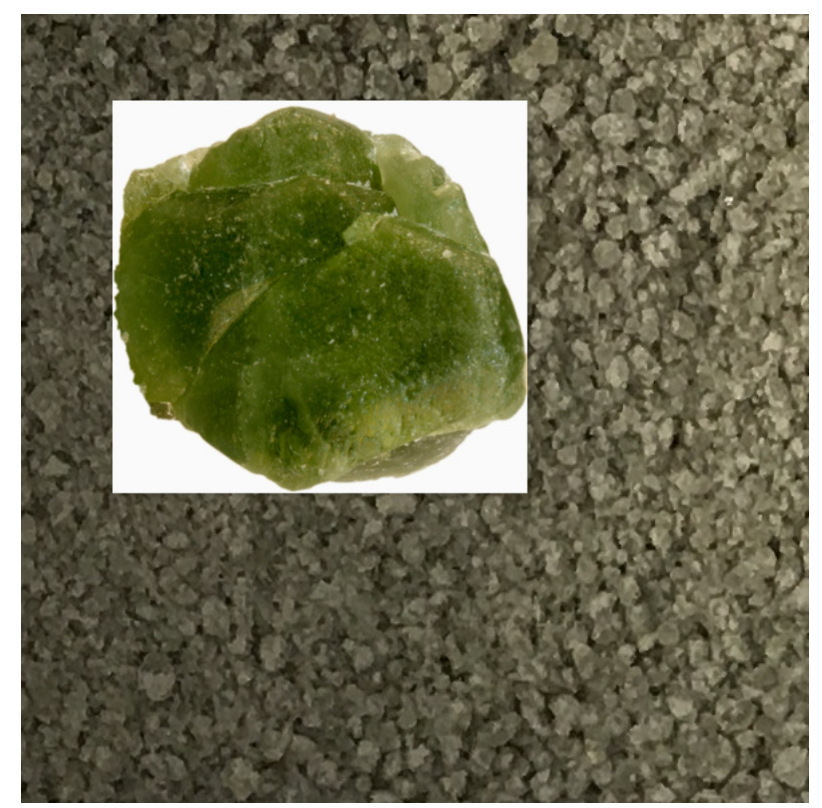

Figure S34 Olivin grains $0.5<d_{p}<0.9 \mathrm{~mm}$

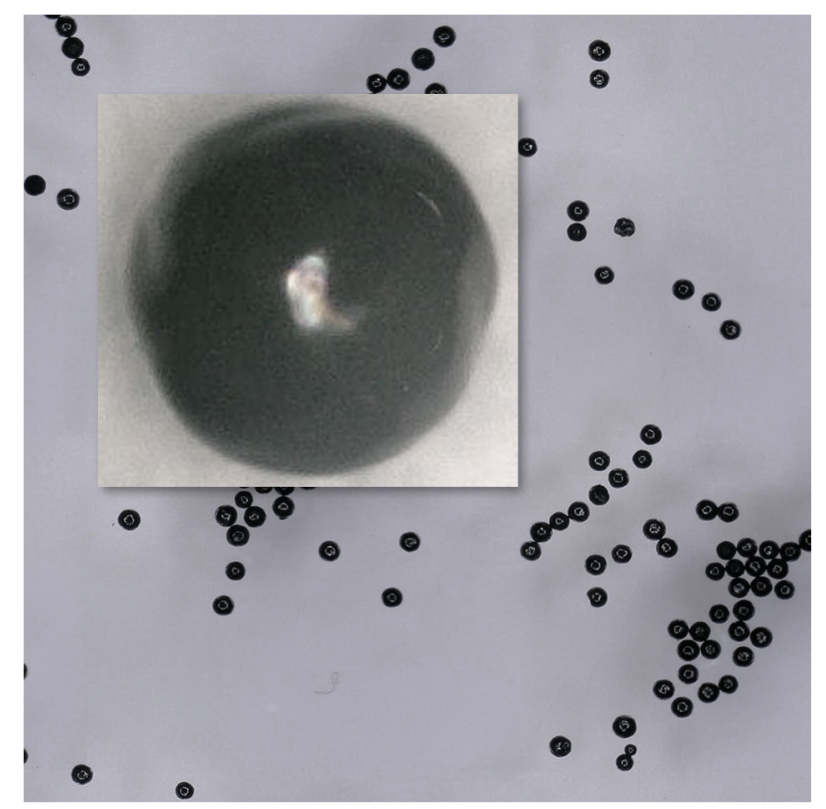

Figure S36 GAC grains (Saratech) $0.44<d_{p}<0.51 \mathrm{~mm}$

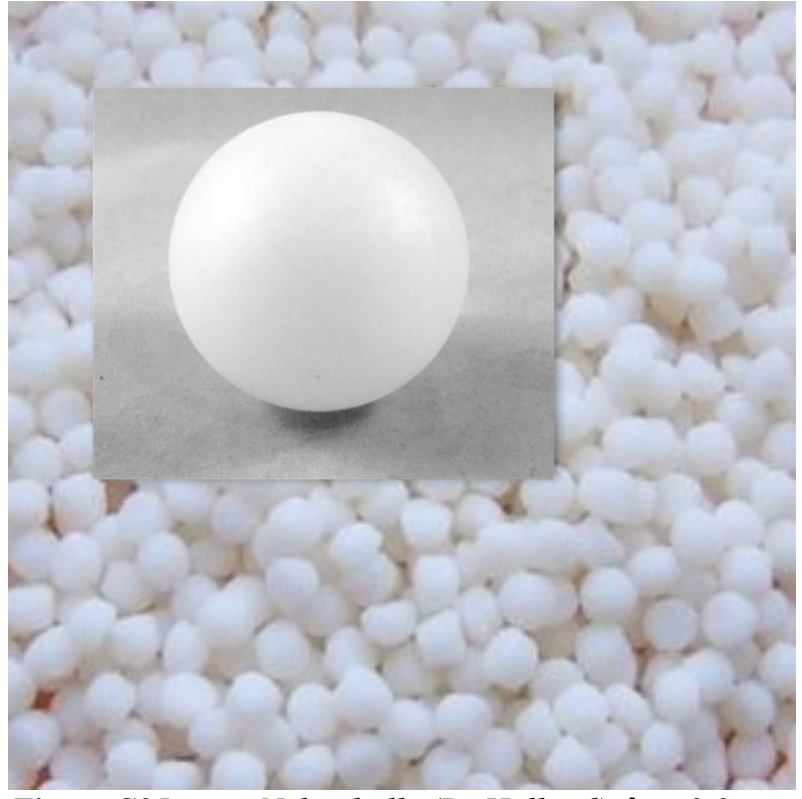

Figure S35 Nylon balls (DitHolland) $d_{p}=3.0 \mathrm{~mm}$

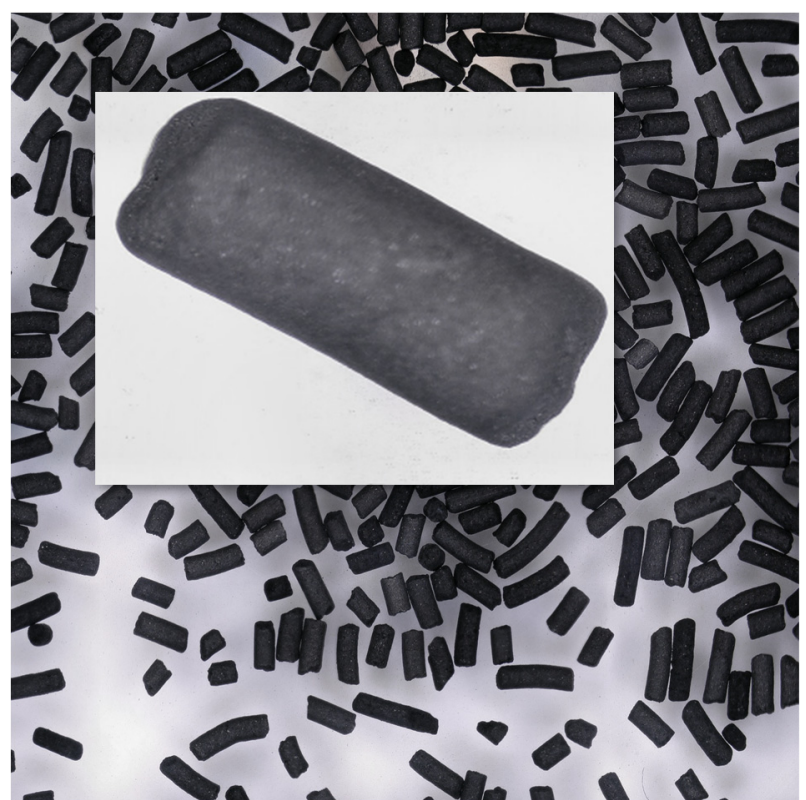

Figure S37 GAC (Norit ROW 0.8 Supra) $0.89<d_{p}<3.15 \mathrm{~mm}$ 


\section{S2.2 Particle selection}

For the terminal settling experiments opaque and transparent spherical polydisperse and monodisperse glass beads were selected (Table S2 and Table S3).In this study, we also examined drinking water related particles, like calcite pellets (100\% $\mathrm{CaCO}_{3}$ ), and crushed calcite seeding material grains (Table $\mathrm{S} 4$ and Table S5), both applied in drinking water softening (Graveland et al., 1983); (Schetters et al., 2015). Polydisperse calcite pellets were sieved and separated to acquire more uniformly dispersed samples. The morphological particle properties obtained with a Camsizer (Retsch-Technology, 2007) show that crushed calcite and the smallest fractionated calcite pellets are relatively more irregularly shaped. The larger the grains become in the softening process due to crystallisation, the more spherically shaped they appear to be.In addition, a wide variety of grains (exotics) with different sizes, shapes and densities were investigated for their terminal settling velocity and behaviour (Table S6).

Table S2 Polydisperse glass pearls ${ }^{1)}$

\begin{tabular}{lll}
\hline Glass colour & $\begin{array}{l}\text { Particle diameter } \\
{[\mathbf{m m}]}\end{array}$ & $\begin{array}{l}\text { Particle density } \\
{\left[\mathbf{k g} / \mathbf{m}^{\mathbf{3}}\right]}\end{array}$ \\
\hline Transparent & $0.25-0.50$ & 2,600 \\
Transparent & $0.50-0.75$ & 2,412 \\
Transparent & $1.18-1.30$ & 2,430 \\
Transparent & $\left.1.25-1.40^{2}\right)$ & 2,550 \\
Transparent & $1.70-2.352)$ & 2,600 \\
Transparent & $\left.2.85-3.15^{2}\right)$ & 2,600 \\
1 B) & \\
2 Boom laboratories BV
\end{tabular}

Table S3 Monodisperse (SiLibeads) glass beads ${ }^{\text {l) }}$

\begin{tabular}{llll}
\hline Glass colour & Type ${ }^{2)}$ & $\begin{array}{l}\text { Particle diameter } \\
{[\mathbf{m m}]}\end{array}$ & $\begin{array}{l}\text { Particle density } \\
{\left[\mathbf{k g} / \mathbf{m}^{\mathbf{3}}\right]}\end{array}$ \\
\hline Transparent & Type P soda lime & 0.7 & 2,655 \\
Transparent & Type P soda lime & 0.8 & 2,588 \\
Blue & Type P matted opaque & 1.0 & 2,550 \\
Green & Type P matted opaque & 1.5 & 2,525 \\
Red & B-707 surface matt & 1.5 & 2,600 \\
Green & B-592 surface matt & 1.5 & 2,600 \\
Green & B-260 surface polished & 2.0 & 2,600 \\
Red & Type P matted opaque & 2.5 & 2,540 \\
Red & B-707 surface polished & 2.5 & 2,600 \\
Black & B-850 surface polished 2.5 & 2,600 \\
Dark blue & B-162 surface polished 3.0 & 2,600 \\
Black & B-850 surface polished 3.0 & 2,600 \\
Green & Type P matted opaque & 3.5 & 2,525 \\
Coral red & B-702 surface polished 3.5 & 2,600 \\
Blue & B-162 surface polished 4.0 & 2,600 \\
Grey & B-386 surface polished 4.0 & 2,600 \\
Transparent & Type G soda lime & 10.0 & 2,500 \\
1) & SiLi Sigmun Linder Gmb &
\end{tabular}

1) SiLi Sigmund Linder $\mathrm{GmbH}$
Deviation given by manufacturer $\pm 20[\mu \mathrm{m}]$ 
Table S4 Fractionated calcite and garnet pellets

\begin{tabular}{llll}
\hline Nr. & Pellet diameter range & $\begin{array}{l}\text { Particle density } \\
\text { calcite pellets } \\
{\left[\mathbf{k g} / \mathbf{m}^{3}\right]}\end{array}$ & $\begin{array}{l}\text { Particle density } \\
\text { garnet pellets } \\
{\left[\mathbf{k g} / \mathbf{m}^{3}\right]}\end{array}$ \\
\hline 1 & $d_{p}<0.355 \mathrm{~mm}$ & 2,670 & 3,807 \\
2 & $0.355<d_{p}<0.425 \mathrm{~mm}$ & 2,670 & 3,069 \\
3 & $0.425<d_{p}<0.5 \mathrm{~mm}$ & 2,658 & 2,908 \\
4 & $0.5<d_{p}<0.63 \mathrm{~mm}$ & 2,670 & 2,802 \\
5 & $0.63<d_{p}<0.71 \mathrm{~mm}$ & 2,650 & 2,748 \\
6 & $0.71<d_{p}<0.8 \mathrm{~mm}$ & 2,650 & 2,721 \\
7 & $0.8<d_{p}<0.9 \mathrm{~mm}$ & 2,663 & 2,708 \\
8 & $0.9<d_{p}<1.0 \mathrm{~mm}$ & 2,649 & 2,697 \\
9 & $1.0<d_{p}<1.12 \mathrm{~mm}$ & 2,634 & 2,690 \\
10 & $1.12<d_{p}<1.25 \mathrm{~mm}$ & 2,664 & 2,685 \\
11 & $1.25<d_{p}<1.4 \mathrm{~mm}$ & 2,666 & 2,680 \\
12 & $1.4<d_{p}<1.7 \mathrm{~mm}$ & 2,657 & 2,676 \\
13 & $1.7<d_{p}<2.0 \mathrm{~mm}$ & 2,657 & 2,674 \\
14 & $2.0<d_{p}<2.36 \mathrm{~mm}$ & 2,655 & 2,672 \\
15 & $2.36<d_{p}<2.8 \mathrm{~mm}$ & 2,657 & 2,671 \\
16 & $d_{p} 2.8<d_{p}<3.35 \mathrm{~mm}$ & 2,657 & 2,671 \\
17 & $d_{p}>3.35 \mathrm{~mm}$ & 2,655 & 2,670 \\
\hline
\end{tabular}

Table S5 Fractionated crushed calcite

Nr. Crushed calcite diameter range Particle density

$\left[\mathrm{kg} / \mathrm{m}^{3}\right]$

\begin{tabular}{lll}
\hline 1 & $0.425<d_{p}<0.5 \mathrm{~mm}$ & 2,582 \\
2 & $0.5<d_{p}<0.61 \mathrm{~mm}$ & 2,579 \\
\hline
\end{tabular}

Table S6 Particle properties exotic grains

\begin{tabular}{|c|c|c|c|c|}
\hline Grain type & $\begin{array}{l}\text { Particle diameter } \\
{[\mathrm{mm}]}\end{array}$ & $\begin{array}{l}\text { Particle density } \\
{\left[\mathrm{kg} / \mathrm{m}^{3}\right]}\end{array}$ & $\begin{array}{l}\text { Sphericity } \\
\text { [-] }\end{array}$ & $\begin{array}{l}\text { Geldarts } \\
\text { type }^{2)}\end{array}$ \\
\hline Crystal sand (AcquaSilica, Kremer Zand \& Grind BV) & $0.40-0.63$ & 2,636 & 0.88 & $\mathrm{~B}$ \\
\hline Olivine (Greensand BV) & $0.50-0.90$ & 3,400 & 0.85 & $\mathrm{~B}$ \\
\hline Mined Italian Calcite & $0.43-0.56$ & 2,575 & 0.84 & $\mathrm{~B}$ \\
\hline Garnet sand sieved fraction & $0.13-0.15$ & 4,376 & 0.84 & $\mathrm{~B}$ \\
\hline '” & $0.15-0.18$ & 4,370 & 0.84 & $\mathrm{~B}$ \\
\hline '” & $0.18-0.21$ & 4,179 & 0.85 & $\mathrm{~B}$ \\
\hline Garnet sand (mesh 80) & $0.21-0.30$ & 4,141 & 0.85 & $\mathrm{~B}$ \\
\hline Garnet sand (softening distortion layer particles) ${ }^{3)}$ & $0.30-0.36$ & 3,026 & 0.85 & $\mathrm{~B}$ \\
\hline Rapid sand filter & $0.80-1.25$ & 2,576 & 0.89 & $\mathrm{D}$ \\
\hline GAC Norit ROW 0.8 Supra (rod) & $0.89-3.15$ & $1,2001)$ & 0.74 & $\mathrm{~B}$ \\
\hline GAC Saratech (spherical) & $0.44-0.51$ & $1,4201)$ & 0.96 & A \\
\hline IEX anionic exchange resins ${ }^{4)}$ & $0.90-1.18$ & 1,090 & 0.95 & A \\
\hline Synthetic material nylon resin (DIT POM balls) & 3.00 & 1,415 & 0.99 & $\mathrm{D}$ \\
\hline \multirow[t]{3}{*}{ Zirconium balls (Baan Machines BV) } & 0.10 & 5,990 & 0.95 & $\mathrm{~B}$ \\
\hline & 1.00 & 5,981 & 0.95 & $\mathrm{D}$ \\
\hline & 2.00 & 5,975 & 0.95 & $\mathrm{D}$ \\
\hline Steel shots $(\mathrm{TU} / \mathrm{e})$ & 3.00 & 7,965 & 0.99 & $\mathrm{D}$ \\
\hline
\end{tabular}

Steel shots (TU/e)

2) Wet density 1 Geldart's particle classification (Geldart, 1973). Type A: aeraable S. B: sand-like. D: spoutable particles

3) Fluffy garnet pellets resulting from deteriorated softening

4) Lewatit VP OC 1071 
Table S7 Data spread determined particle density (NEN-EN 933-2, n.d.)

\begin{tabular}{|c|c|c|}
\hline Grain type & Error & Method \\
\hline Glass pearls & $3.5 \%$ & $1,2,3,4,5$ \\
\hline SiLibeads & $1.5 \%$ & $1,2,3,4,5$ \\
\hline Crushed calcite & $3.8 \%$ & 1,4 \\
\hline Synthetic material & $5.0 \%$ & $1,4,5$ \\
\hline Calcite pellets & $2.2 \%$ & 1,4 \\
\hline Garnet pellets & $6.8 \%$ & 1,4 \\
\hline Crystal sand (AcquaSilica, Kremer Zand \& Grind BV) & $6.0 \%$ & $1,4,5$ \\
\hline Olivine (Greensand BV) & $7.5 \%$ & 5 \\
\hline Mined Italian Calcite & $3.8 \%$ & 1,5 \\
\hline Garnet sand (mesh 80) & $4.5 \%$ & 1,5 \\
\hline Rapid sand filter & $4.3 \%$ & 1,4 \\
\hline Granular Activated Carbon & $7.8 \%$ & 1,5 \\
\hline IEX anionic exchange resins (Lewatit VP OC 1071) & $3.0 \%$ & $1,2,5$ \\
\hline Synthetic material nylon resin (DIT POM balls) & $3.0 \%$ & $1,2,4,5$ \\
\hline Zirconium balls (Baan Machines BV) & $1.7 \%$ & 4,5 \\
\hline Steel shots (TU/e) & $1.5 \%$ & $1,2,4$ \\
\hline $1 \quad$ Pycnometer & & \\
\hline Particle count & & \\
\hline ImageJ & & \\
\hline Differential pressure & & \\
\hline Supplier & & \\
\hline
\end{tabular}




\section{S2.3 Average particle diameter}

The effective or average hydraulic equivalent particle diameter $d_{p}$ of calcite pellets was based on the applied sieve method in which particles were divided over the slides of sieves and calculated according to the appropriate geometric mean for two sieves. The distance between successive sieve openings varied between $2, \sqrt{ } 2,1.20$ and 1.12. Available sieve sizes are usually regulated by standards such as ISO 3310-1, ISO 565, EN 933-2 and ASTM E11 (NEN-EN 933-2, n.d.).In this research, the ratios between two successive sieve openings were 1.12 and 1.20. The hydraulic equivalent particle diameter, based on the geometric mean (Davis, 2010) was calculated using Equation (S2):

$$
d_{p}=\sqrt{d_{s, 1} d_{s, 2}}
$$

The particle size of glass beads (SiLibeads) was determined through static image analysis. Based on a scan of a sample using specialised software such as ImageJ that analyses pixels, it is possible to compute the size and different morph parameters of each particle in the picture. Particle size determination using ImageJ is occasionally more complex, caused by Image properties aspects like threshold and pixels.

$$
d_{p}=2 \sqrt{\frac{\sum_{i}^{N} A_{i}}{N \pi}}
$$

Table S8 shows particle sizes and the number of individual fluidisation experiments for perfectly round spheres and natural gratins applied in water treatment processes. 
Table S8 Determined particle sizes and types

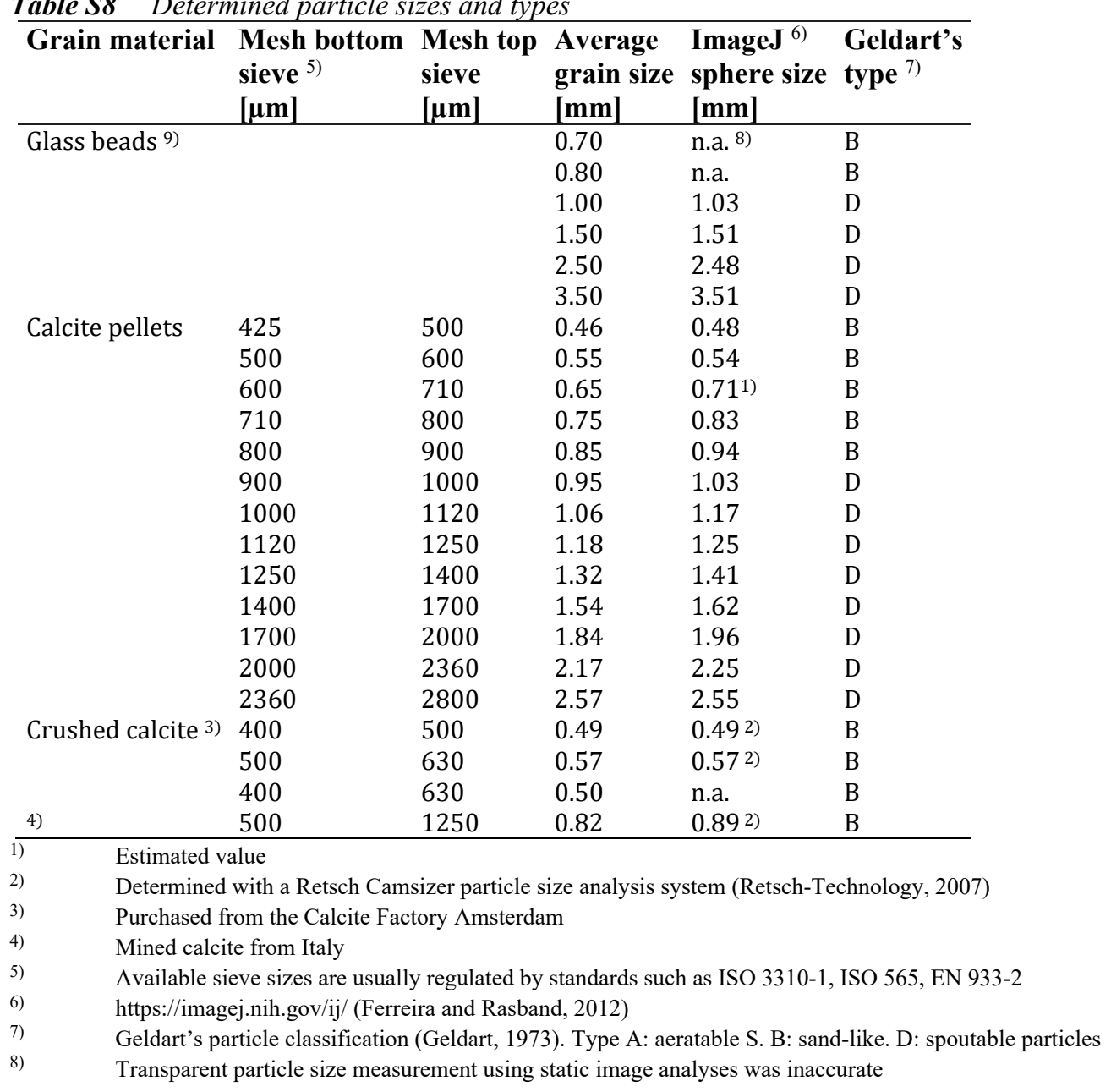

The particle size of calcite pellets and crushed calcite determined with ImageJ is slightly higher (Table S8) compared to the average sieve diameter: $\left(R^{2}=0.994\right)$. This can be explained by the fact that pellets and calcite are irregularly shaped due to the grinding process and therefore have a tendency to pass sieves more vertically oriented, while they are more horizontally oriented during static image analysis where particles are lying on a scanner. In case of calcite pellets, for modelling purposes, the average hydraulic equivalent particle diameter $d_{p}$ is used (Equation (S2)). 


\section{S2.4 Particle density}

The particle density was determined with the help of several methods, based on particle counting, static image analysis, differential pressure and/or pycnometer measurements.

\section{S2.4.1 Particle counting}

A straightforward way to calculate the particle density is particle counting with Equation (S4):

$$
\rho_{p}=\frac{\sum_{i}^{N} m_{i}}{N \frac{\pi}{6} d_{p}{ }^{3}}
$$

\section{S2.4.2 Static image analyses}

Using ImageJ software (Ferreira and Rasband, 2012), the particle density can also be calculated when combined with a mass measurement.

\section{S2.4.3 Differential pressure}

The particle density was also obtained through fluidisation experiments (Kramer et al., 2020a); (Kramer et al., 2020b). Based on the total particle mass and differential pressure sensor value of the experimental set-up, where the pressure drop in a homogenous fluidisation state is independent of the prevalent superficial fluid velocity, the particle density can be determined using Equation (S5).

$$
\begin{aligned}
& \Delta P=\frac{m g}{\frac{\pi}{4} D^{2}} \frac{\left(\rho_{p}-\rho_{f}\right)}{\rho_{p}} \\
& \Delta P=\rho_{f} g \Delta H
\end{aligned}
$$

\section{S2.4.4 Laboratory pycnometer}

A traditional laboratory tool to measure particle density is the pycnometer. The particle density of grain material was measured with a $50 \mathrm{~mL}$ pycnometer.

\section{S2.4.5 Grains with different densities}

Due to crystallisation of $\mathrm{CaCO}_{3}$ at the particle surface (van der Veen and Graveland, 1988); (Kramer et al., 2019), the particle size increases in time. Since the density of the seeding material, for instance garnet grains, is different from the density of calcium carbonate, the average density changes during the softening process. Equation 7 was used to estimate average particle density for garnet pellets based on the assumption that round particles contain an equally distributed layer of pure chalk with a density of $2,711 \mathrm{~kg} / \mathrm{m}^{3}$, as postulated by Anthony (Anthony et al., 2003).

$$
d_{p}{ }^{3} \rho_{p}=d_{g}{ }^{3} \rho_{g}+\left(d_{p}{ }^{3}-d_{g}{ }^{3}\right) \rho_{c}
$$




\section{S2.4.6 Density measurement results}

The particle densities were determined through different methods and are given in Table S3 and Figure S38. For calcite pellets, the pycnometer density is the most accurate (average relative error $=0.8 \%$ ) compared to the derived density based on the differential pressure sensor value, using Equation (S5) (average relative error $=4.5 \%$ ). For modelling purposes, the determined mean value was used.

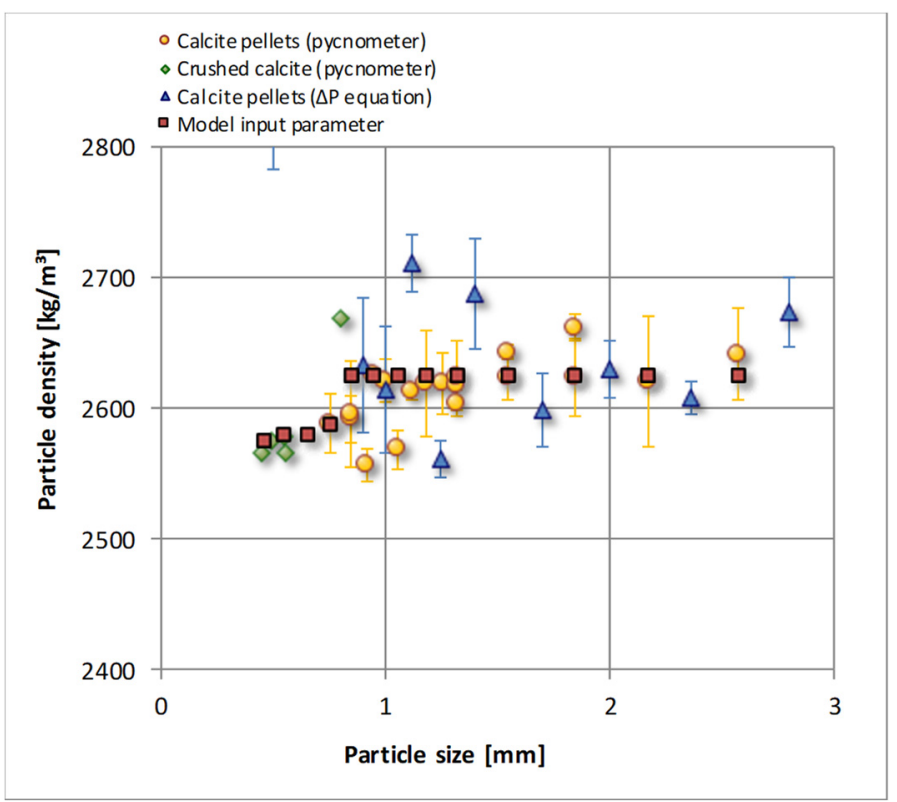

Figure S38 Particle densities of drinking water pellet-softening grains

\section{S2.5 Particle characterisation and morphological properties}

Particle characterisation is taken from (Kramer et al., 2020a) (Kramer et al., 2020b).

The following morphological properties are defined:

$d_{i} \quad$ Effective size of a sample where $i$ percentage of particles is smaller than the particular size [m]

$E_{H, 50}$ Ellipsoid height (cumulative 50\% point) [m]

$E_{L, 50} \quad$ Ellipsoid length (cumulative 50\% point) [m]

$E_{W, 50} \quad$ Ellipsoid width (cumulative 50\% point) [m]

UC Non-uniformity coefficient $d_{60} / d_{10}$

$\Phi \quad$ Sphericity: ratio between surface area of the volume equivalent sphere and considered particle $\frac{\pi^{\frac{1}{3}}\left(6 V_{p}\right)^{\frac{2}{3}}}{A_{S}} \quad[-]$

$\Xi \quad$ Circularity calculated from the perimeter $\mathrm{P}$ and area $\mathrm{A}$ of the particle projection $\sqrt{\frac{4 \pi A_{p}}{P^{2}}} \quad[-]$

Symm Symmetry, the distances between the centre of area to the particle projection borders [-] 


\begin{tabular}{|c|c|c|c|c|c|c|c|c|c|c|c|c|c|c|}
\hline Grain type & Diameter & $\begin{array}{l}d_{s, \min } \\
{[\mathrm{mm}]}\end{array}$ & $\begin{array}{l}d_{s, \max } \\
{[\mathrm{mm}]}\end{array}$ & $\begin{array}{l}d_{p} \\
{[\mathrm{~mm}]}\end{array}$ & $\begin{array}{l}d_{10} \\
{[\mathrm{~mm}]}\end{array}$ & $\begin{array}{l}d_{50} \\
{[\mathrm{~mm}]}\end{array}$ & $\begin{array}{l}d_{90} \\
{[\mathrm{~mm}]}\end{array}$ & $\begin{array}{l}U C \\
{[-]}\end{array}$ & $\begin{array}{l}\Phi \\
{[-]}\end{array}$ & $\begin{array}{l}\Xi \\
{[-]}\end{array}$ & $\begin{array}{l}\text { Symm } \\
{[-]}\end{array}$ & $\begin{array}{l}E_{L, 50} \\
{[\mathrm{~mm}]}\end{array}$ & $\begin{array}{l}E_{W, 50} \\
{[\mathrm{~mm}]}\end{array}$ & $\begin{array}{c}E_{H, 50} \\
{[\mathrm{~mm}]}\end{array}$ \\
\hline Calcite pellets & $0.50-0.63$ & 0.50 & 0.63 & 0.56 & 0.54 & 0.64 & 0.74 & 1.21 & 0.90 & 0.86 & 0.92 & 0.80 & 0.59 & 0.33 \\
\hline “” & $0.63-0.71$ & 0.63 & 0.71 & 0.67 & 0.64 & 0.73 & 0.83 & 1.17 & 0.91 & 0.86 & 0.92 & 0.95 & 0.70 & 0.50 \\
\hline “” & $0.80-0.90$ & 0.80 & 0.90 & 0.85 & 0.79 & 0.90 & 1.00 & 1.17 & 0.94 & 0.86 & 0.93 & 1.05 & 0.83 & 0.66 \\
\hline '” & $0.90-1.00$ & 0.90 & 1.00 & 0.95 & 0.88 & 0.99 & 1.10 & 1.15 & 0.95 & 0.87 & 0.93 & 1.13 & 0.93 & 0.78 \\
\hline “” & $1.00-1.12$ & 1.00 & 1.12 & 1.06 & 1.02 & 1.15 & 1.29 & 1.15 & 0.96 & 0.87 & 0.94 & 1.22 & 1.02 & 0.87 \\
\hline '” & $1.12-1.25$ & 1.12 & 1.25 & 1.18 & 1.11 & 1.23 & 1.35 & 1.14 & 0.96 & 0.87 & 0.94 & 1.25 & 1.04 & 0.90 \\
\hline “” & $1.25-1.40$ & 1.25 & 1.40 & 1.32 & 1.22 & 1.37 & 1.53 & 1.15 & 0.97 & 0.87 & 0.94 & 1.35 & 1.14 & 0.97 \\
\hline '” & $1.40-1.70$ & 1.40 & 1.70 & 1.54 & 1.41 & 1.58 & 1.76 & 1.15 & 0.98 & 0.86 & 0.94 & 1.38 & 1.16 & 0.99 \\
\hline "” & $1.70-2.00$ & 1.70 & 2.00 & 1.84 & 1.72 & 1.90 & 2.10 & 1.13 & 0.98 & 0.86 & 0.95 & 1.41 & 1.21 & 1.06 \\
\hline '” & $2.00-2.36$ & 2.00 & 2.36 & 2.17 & 2.04 & 2.22 & 2.42 & 1.11 & 0.98 & 0.86 & 0.96 & 1.43 & 1.23 & 1.12 \\
\hline '” & $2.36-2.80$ & 2.36 & 2.80 & 2.57 & 2.27 & 2.50 & 2.75 & 1.12 & 0.99 & 0.82 & 0.96 & 1.61 & 1.39 & 1.25 \\
\hline “" & $>2.80$ & 2.80 & 3.35 & 3.06 & 2.72 & 2.97 & 3.30 & 1.11 & 0.99 & 0.82 & 0.96 & 1.80 & 1.57 & 1.50 \\
\hline Crushed calcite & $0.40-0.50$ & 0.40 & 0.50 & 0.45 & 0.39 & 0.49 & 0.58 & 1.28 & 0.84 & 0.78 & 0.88 & 0.73 & 0.52 & 0.29 \\
\hline & $0.50-0.60$ & 0.50 & 0.60 & 0.55 & 0.45 & 0.57 & 0.68 & 1.31 & 0.84 & 0.78 & 0.89 & 0.73 & 0.52 & 0.29 \\
\hline
\end{tabular}

The sphericity for crushed calcite and calcite pellets can be interpolated using an empirical Morgan-Mercer-Flodin growth model with Equation (S8).

$$
\phi_{s}=\frac{c_{0} c_{1}+c_{2} d_{p}{ }^{c_{3}}[\mathrm{~mm}]}{c_{1}+d_{p}{ }^{c_{3}}[\mathrm{~mm}]}
$$

$$
\left(d_{p}[\mathrm{~mm}]>0.4\right)
$$

Table S10 Fitting parameters $M M F$

\begin{tabular}{lc}
\multicolumn{2}{c}{ model $R^{2}=0.98$} \\
\hline Coefficient & Value \\
& \\
\hline$c_{0}$ & 0.728 \\
$c_{1}$ & 0.171 \\
$c_{2}$ & 0.986 \\
$c_{3}$ & 3.000 \\
\hline
\end{tabular}

The sphericity of garnet pellets was not analysed. Nevertheless, the sphericity is assumed to be approximately the same as sphericity for calcite pellets. In fact, it is likely that the sphericity of garnet pellets is slightly higher compared to the sphericity of calcite pellets since its garnet core is smaller and therefore the $\mathrm{CaCO}_{3}$ layer can become more spherical in an early state.

\section{S3 Calculation of the local gravitational field of earth}

The local gravitational field of earth $g$ is dependent on the degree of latitude, as indicated by (Stevens et al., 2016) and can be calculated with Equation (S9):

$$
g=g_{45}-\frac{1}{2}\left(g_{\text {poles }}-g_{\text {equator }}\right)+\cos \left(2 \text { lat } \frac{\pi}{180}\right)
$$

where $g_{\text {poles }}=9.780 \mathrm{~m} / \mathrm{s}^{2}, g_{45}=9.806 \mathrm{~m} / \mathrm{s}^{2}, g_{\text {equator }}=9.832 \mathrm{~m} / \mathrm{s}^{2}$ and $l a t=$ latitude between $-90^{\circ}$ and $90^{\circ}$. 
Wall effects are taken from the Discussion section in the Supplementary Material section in (Kramer et al., 2020b).

In the literature, many wall effect correction formulas can be found. These wall effects refer to the retardation of the motion of individual particles settling in a cylinder due to the displacement and opposing motion of the surrounding fluid (Clift et al., 1978); (Yang, 2003). Wall effects depend on the ratio of the particle diameter to the cylinder column diameter $d_{p} / D$ and the sphere Reynolds number $R e_{t}$ for terminal settling conditions (di Felice, 1995); (di Felice and Gibilaro, 2004); (Chhabra et al., 2003).

In the literature, correction formulas are also given (Richardson and Zaki, 1954); (Loeffler, 1953), often developed on an empirical basis. The effect of container walls, however, can be neglected in most full-scale operations (Akgiray and Soyer, 2006). Regarding relatively small laboratory columns, wall effects might have to be considered because wall friction opposes the drag force acting on the particles during fluidisation. Numerous researchers, including (Loeffler, 1953), (Dharmarajah, 1982), (Khan and Richardson, 1989), (di Felice, 1995), (Rao et al., 2010), (Whiten and Özer, 2015) and (do Nascimento et al., 2016) have proposed empirical equations based on particle-to-column ratio, Reynolds number or velocity ratio, to apply corrections. According to (Fidleris and Whitmore, 2002) the retarding effect of the wall decreases with increasing terminal Reynolds number, so that for ratios of $\left(d_{p} / D=0.05-0.10\right)$ the wall correction becomes less than $1 \%$ for Reynolds numbers exceeding 5 and 30, respectively. However, there is no general agreement in the literature. A straightforward critical value for particle-to-column ratio varies between 0.06 and 0.2 . When we take $\left(d_{p} / D\right)_{c r i t}$, then for almost all experiments corrections are not needed. Wall effects are only significant for large glass beads $d_{p}=10$ [mm]. (Fidleris and Whitmore, 2002) proposed the improved Ladenburg wall effects correction Equation (SEquation (S10)):

$$
k=1-2.104 \frac{d_{p}}{D}+2.09\left(\frac{d_{p}}{D}\right)^{3}+0.95\left(\frac{d_{p}}{D}\right)^{5} \quad\left(d_{p} / D<2\right)
$$

where

$$
k=\frac{v_{t}}{v_{t, \infty}}
$$

(Arsenijević et al., 2010) and (Chhabra et al., 2003) suggested the Haberman equation (Haberman and Sayre, 1958) Equation (S12), which has gained wide acceptance in the literature:

$$
k=\frac{1-2.104 \frac{d_{p}}{D}+2.0865\left(\frac{d_{p}}{D}\right)^{3}-1.7068\left(\frac{d_{p}}{D}\right)^{5}+0.72603\left(\frac{d_{p}}{D}\right)^{6}}{1-0.75857\left(\frac{d_{p}}{D}\right)^{5}}
$$

which can be simplified for the uncertainty analysis:

$$
k=1-2.104 \frac{d_{p}}{D}
$$




\section{S5 Drag coefficient prediction models from the literature}

\section{S5.1 Spherical particles}

Table S11 Drag coefficient prediction models from the literature for spherical particles

\begin{tabular}{|c|c|c|c|}
\hline Reference & Equation & Boundary conditions & $\begin{array}{l}\text { Eq. } \\
\text { nr. }\end{array}$ \\
\hline (Stokes, 1850) & $C_{D}=\frac{24}{R e_{t}}$ & $R e_{t}<0.1$ & (S14) \\
\hline (Newton, 1726) & $C_{D}=0.44$ & $750<R e_{t}<350,000$ & $(\mathrm{~S} 15)$ \\
\hline (Schiller and Naumann, 1933) & $C_{D}=\frac{24}{R e_{t}}\left(1+0.15 R e_{t}^{0.681}\right)$ & $R e_{t}<800$ & $(\mathrm{~S} 16)$ \\
\hline (Dallavalle, 1948) & $C_{D}=\left(0.63+\frac{4.8}{\sqrt{R e_{t}}}\right)^{2}$ & $0.015<R e_{t}<3,000$ & $(\mathrm{~S} 17)$ \\
\hline (Fair et al., 1954) & $C_{D}=\frac{24}{R e_{t}}+\frac{4}{\sqrt{R e_{t}}}+0.34$ & $R e_{t}<100,000$ & $(\mathrm{~S} 18)$ \\
\hline (Clift and Gauvin, 1971) & $C_{D}=\frac{24}{R e_{t}}\left(1+0.15 R e_{t}^{0.687}\right)+\frac{0.42}{1+\frac{42500}{R e_{t}^{1.16}}}$ & $R e_{t}<300,000$ & $(\mathrm{~S} 19)$ \\
\hline \multirow[t]{7}{*}{ (Clift et al., 1978) } & $C_{D}=\frac{24}{R e_{t}}+\frac{3}{16}$ & $R e_{t}<0.1$ & $(\mathrm{~S} 20)$ \\
\hline & $C_{D}=\frac{24}{R e_{t}}\left(1+0.1315 R e_{t}^{\left(0.82-0.05 \log R e_{t}\right)}\right)$ & $0.01<R e_{t}<20$ & $(\mathrm{~S} 21)$ \\
\hline & $C_{D}=\frac{24}{R e_{t}}\left(1+0.1935 R e_{t}^{0.6305}\right)$ & $20<R e_{t}<260$ & $(\mathrm{~S} 22)$ \\
\hline & $\log C_{D}=1.6435-1.1242 \log R e_{t}+0.1558\left(R e_{t}\right)^{2}$ & $260<R e_{t}<1,500$ & $(\mathrm{~S} 23)$ \\
\hline & $\log C_{D}=-2.4571+2.5558 \log R e_{t}-0.9295\left(R e_{t}\right)^{2}+0.1049\left(R e_{t}\right)^{3}$ & $1,500<R e_{t}<12,000$ & $(\mathrm{~S} 24)$ \\
\hline & $\log C_{D}=-1.9181+0.6370 \log R e_{t}-0.0636\left(R e_{t}\right)^{2}$ & $12,000<R e_{t}<44,000$ & $(\mathrm{~S} 25)$ \\
\hline & $\log C_{D}=-4.3390+1.5809 \log R e_{t}-0.1546\left(R e_{t}\right)^{2}$ & $44,000<R e_{t}<338,000$ & $(\mathrm{~S} 26)$ \\
\hline (Graf, 1984) & $C_{D}=\frac{24}{R e_{t}}+\frac{7.3}{1+\sqrt{R e_{t}}}+0.25$ & $R e_{t}<200,000$ & $(\mathrm{~S} 27)$ \\
\hline (Turton and Levenspiel, 1986) & $C_{D}=\frac{24}{R e_{t}}\left(1+0.17 R e_{t}^{0.657}\right)+\frac{0.413}{1+\frac{16300}{R e_{t}^{1.09}}}$ & $R e_{t}<260,000$ & $(\mathrm{~S} 28)$ \\
\hline \multirow[t]{2}{*}{ (Flemmer and Banks, 1986) } & $C_{D}=\frac{24}{R e_{t}} 10^{E}$ & $R e_{t}<86,000$ & $(\mathrm{~S} 29)$ \\
\hline & $E=0.261 R e_{t}^{0.369}-0.105 R e_{t}^{0.431}-\frac{0.124}{1+\log \left(R e_{t}\right)^{2}}$ & & \\
\hline (Khan and Richardson, 1987) & $C_{D}=\left(2.25 R e_{t}^{-0.31}+0.36 R e_{t}^{0.06}\right)^{3.45}$ & $0.01<R e_{t}<300,000$ & $(\mathrm{~S} 30)$ \\
\hline (Haider and Levenspiel, 1989) & $C_{D}=\frac{24}{R e_{t}}\left(1+0.1806 R e_{t}^{0.6459}\right)+\frac{0.4251}{1+\frac{6880.95}{R e_{t}}}$ & $100<R e_{t}<260,000$ & (S31) \\
\hline (Brown and Lawler, 2003) & $C_{D}=\frac{24}{R e_{t}}\left(1+0.15 R e_{t}^{0.681}\right)+\frac{0.407}{1+\frac{8710}{R e_{t}}}$ & $R e_{t}<200,000$ & (S32) \\
\hline (van Schagen et al., 2008) & $C_{D}=\frac{24}{R e_{t}}\left(1+0.079 R e_{t}^{0.67}\right)$ & $R e_{t}<800$ & (S33) \\
\hline (Cheng, 2009) & $C_{D}=\frac{24}{R e_{t}}\left(1+0.27 R e_{t}\right)^{0.43}+0.47\left(1-e^{-0.04 R e_{t} e^{0.38}}\right)$ & $0.002<R e_{t}<200,000$ & (S34) \\
\hline$\left(\right.$ Morrison, 2013) ${ }^{1)}$ & $C_{D}=\frac{24}{R e_{t}}+\frac{2.6\left(\frac{R e_{t}}{5}\right)}{1+\left(\frac{R e_{t}}{5}\right)^{1.52}}+\frac{0.411\left(\frac{R e_{t}}{263,000}\right)^{-7.94}}{1+\left(\frac{R e_{t}}{263,000}\right)^{-8}}+\frac{0.25\left(\frac{R e_{t}}{10^{6}}\right)}{1+\left(\frac{R e_{t}}{10^{6}}\right)}$ & $R e_{t}<1,000,000$ & (S35) \\
\hline
\end{tabular}

Model improvements are proposed with fitted parameters by (Haider and Levenspiel, 1989) 


\section{S5.2 Non-spherical particles}

The sphericity $\Phi$ represents the ratio between the surface area of the volume equivalent sphere and that of the considered particle.

Table S12 Drag coefficient prediction models from the literature for non-spherical particles with sphericity

\begin{tabular}{|c|c|c|c|}
\hline Reference & Equation & Boundary conditions & $\begin{array}{l}\text { Eq. } \\
\text { nr. }\end{array}$ \\
\hline \multirow[t]{5}{*}{ (Haider and Levenspiel, 1989) } & $C_{D}=\frac{24}{R e_{t}}\left(1+c_{1} R e_{t}^{c_{2}}\right)+\frac{c_{3}}{1+\frac{C_{4}}{R e_{t}}}$ & $R e_{t}<300,000$ & (S36) \\
\hline & $c_{1}=\exp \left(2.3288-6.4581 \Phi+2.4486 \Phi^{2}\right)$ & & (S37) \\
\hline & $c_{2}=0.0964+0.5565 \Phi$ & & (S38) \\
\hline & $c_{3}=\exp \left(4.905-13.8944 \Phi+18.4222 \Phi^{2}-10.2599 \Phi^{3}\right)$ & & $(\mathrm{S} 39)$ \\
\hline & $\mathrm{c}_{4}=\exp \left(1.4681+12.2584 \Phi-20.7332 \Phi^{2}+15.8855 \Phi^{3}\right)$ & & $(\mathrm{S} 40)$ \\
\hline \multirow[t]{3}{*}{ (Ganser, 1993) } & $C_{D}=c_{2}\left(\frac{24}{c_{1} c_{2} R e_{t}}\left(1+0.118\left(c_{1} c_{2} R e_{t}\right)^{0.6567}\right)+\frac{0.4305}{1+\frac{3305}{c_{1} C_{2} R e_{t}}}\right)$ & & $(\mathrm{S} 41)$ \\
\hline & $c_{1}=\left(\frac{1}{3}+\frac{2}{3} \Phi^{-0.5}\right)^{-1}$ & & $(\mathrm{~S} 42)$ \\
\hline & $c_{2}=10^{1.8148\left(-^{10} \log (\Phi)\right)^{0.5743}}$ & & $(\mathrm{~S} 43)$ \\
\hline (Chien, 1994) & $C_{D}=\frac{30}{R e_{t}}+\frac{67.289}{e^{5.03 \Phi}}$ & & $(\mathrm{S} 44)$ \\
\hline
\end{tabular}




\section{S5.3 More complex prediction models using more morphological properties}

The crosswise sphericity $\Phi_{\perp}$ is defined as the ratio between the cross-sectional area of the volume equivalent sphere and the projected cross-sectional area of the considered particle perpendicular to the flow. The lengthwise sphericity $\Phi_{\|}$is the ratio between the cross-sectional area of the volume equivalent sphere and the difference between half the surface area and the mean projected longitudinal cross-sectional area of the considered particle (Leith, 1987); (Hölzer and Sommerfeld, 2008).

Table S13 Drag coefficient advanced prediction models from the literature for non-spherical particles

\begin{tabular}{ll}
\hline Reference & Equation \\
\hline (Hölzer and Sommerfeld, 2008) & $C_{D}=\frac{8}{R e_{t}} \frac{1}{\sqrt{\Phi_{\|}}}+\frac{16}{R e_{t}} \frac{1}{\sqrt{\Phi}}+\frac{3}{\sqrt{R e_{t}}} \frac{1}{\Phi_{4}^{3}}+\frac{0.42}{\Phi_{\perp}} 10^{0.4\left(-{ }^{-10} \log (\Phi)\right)^{0.2}}$ \\
$C_{D}$ & $=\frac{24 K_{S}}{R e_{t}}\left(1+0.125\left(R e_{t} \frac{K_{N}}{K_{S}}\right)^{\frac{2}{3}}\right)+\frac{0.46 K_{N}}{1+\frac{5330}{R e_{t}} \frac{K_{N}}{K_{S}}}$ \\
(Bagheri and Bonadonna, 2016) & $K_{S}=\frac{F_{S}^{\frac{1}{3}}+F_{S}^{-\frac{1}{3}}}{2}$ \\
$K_{N}$ & $=10^{\alpha_{2}\left(-{ }^{10} \log \left(F_{N}\right)\right)^{\beta_{2}}}$ \\
$F_{S}$ & $=f e^{1.3}\left(\frac{d_{p}{ }^{3}}{D_{L} D_{M} D_{S}}\right)$ \\
$F_{N}$ & $=f^{2} e\left(\frac{d_{p}{ }^{3}}{D_{L} D_{M} D_{S}}\right)$ \\
$\alpha_{2}$ & $=0.45+\frac{10}{e^{2.5 \log \left(\frac{\rho_{p}}{\rho_{f}}\right)+30}}$ \\
$\beta_{2}$ & $=1-\frac{37}{e^{3 \log \left(\frac{\rho_{p}}{\rho_{f}}\right)+100}}$ \\
$C_{D}$ & $=\frac{24}{R e_{t}}\left(\frac{1-\Psi}{R e_{t}}+1\right)^{\frac{1}{4}}+\frac{24}{R e_{t}}\left(0.1806 R e_{t}{ }^{0.6459}\right) \Psi^{-\left(R e_{t} .08\right)}$ \\
(Dioguardi et al., 2018) & $+\frac{0.4251}{1+\frac{6,880.95}{R e_{t}} \Psi 5.05}$
\end{tabular}

Parameters $f$ and e are the particle flatness and elongation defined by (Dioguardi and Mele, 2015). (Dioguardi et al., 2018) defined a particle shape descriptor as a function of sphericity and circularity as follows:

$$
\Psi=\frac{\Phi}{\Xi}
$$

According to (Dioguardi et al., 2018) and (Serway and Jewett, 2014) a less accurate approximation is the following:

$$
\Psi=0.83 \Phi
$$




\section{S5.4 Brown-Lawler model}

The Brown-Lawler equation is based on the implicit Schiller-Naumann equation (Schiller and Naumann, 1933) in which an extra term is appended to cover the whole range. The Brown-Lawler equation (Brown and Lawler, 2003) is the arbitrary composition of the individual terms for the manifold flow regimes, i.e. the laminar, transitional and turbulent flow regimes:

$C_{D}=t_{1}+t_{2}+t_{3}$

$t_{1}=\frac{24}{R e_{t}}$

laminar regime

$t_{2}=3.60 R e_{t}^{-0.319}$

transitional regime

$t_{3}=\frac{0.407}{1+\frac{8710}{R e_{t}}}$

turbulent regime

$C_{D}=\frac{24}{R e_{t}}\left(1+0.15 R e_{t}^{0.681}\right)+\frac{0.407}{1+\frac{8710}{R e_{t}}}$

$\left(R e_{t}<300,000\right)$

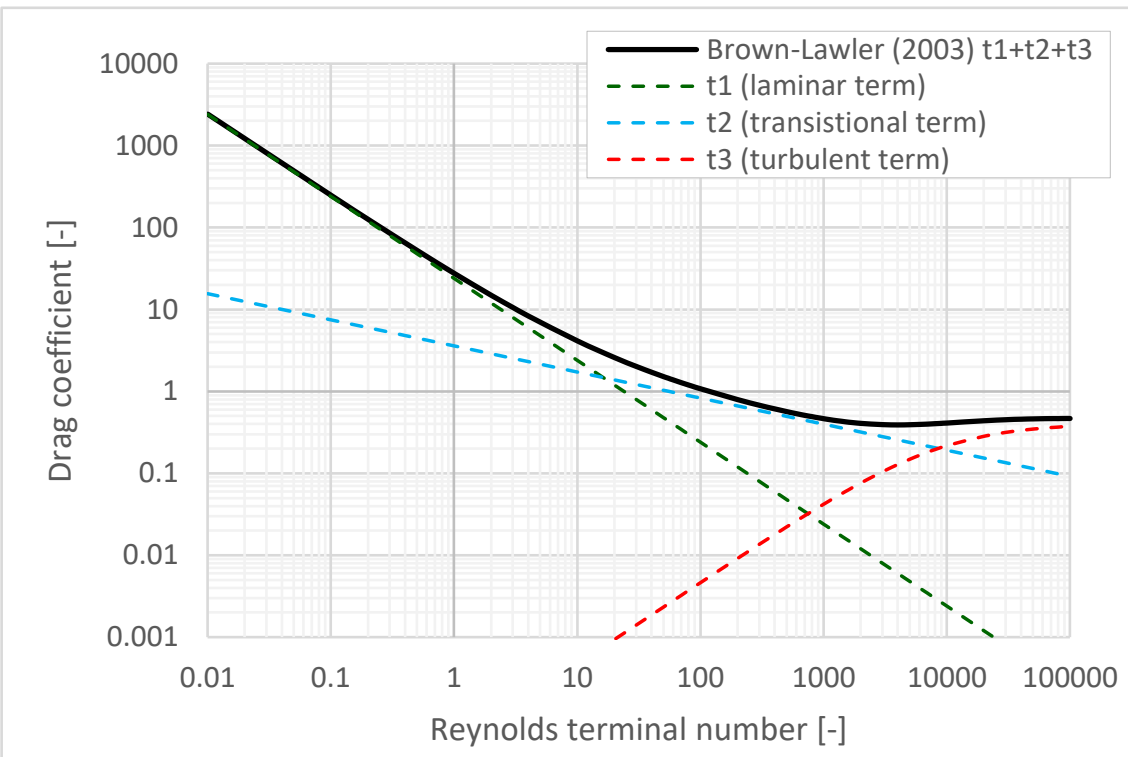

Figure S39 Brown-Lawler model 


\section{S5.5 Range analysis}

Brown and Lawler conducted an elaborate study on drag and settling velocity correlations, and they compared these with selected datasets from the literature. Their preliminary dataset $(N>606)$ was conscientiously filtered in view of a lack of precision, wall effects, non-spherical shapes and limiting Reynolds numbers, to mention but a few aspects. Subsequently, on a definite dataset containing 480 points, they presented the fit to corrected data for both drag and settling velocity correlations. They compared several customary statistical methods. Based on the range analysis method, i.e. the percentage of data points within a specified range of the correlation, they considered Equation S32 to be the most accurate. They presented their statistical results in a tabular form. In the current work, however, their results are plotted graphically, as shown in Figure S40. Brown and Lawler then discussed why the Fair-Geyer equation for drag should be abandoned in favour of the direct calculation available from new proposed models. In Figure S40, it is apparent that the accuracy of the Fair-Geyer equation is significantly lower compared with other models, except for the equation proposed by Brown and Lawler, which only has a slightly higher accuracy. The disadvantage of the latter, however, is the division of the terminal settling velocity into ten different segments, each valid for a specific Reynolds range. Figure S40 shows that the Fair-Geyer equation can be rejected in favour of the Brown-Lawler equation. However, the observed difference in accuracy between Brown-Lawler and Fair-Geyer presented in the statistical methods is marginal.

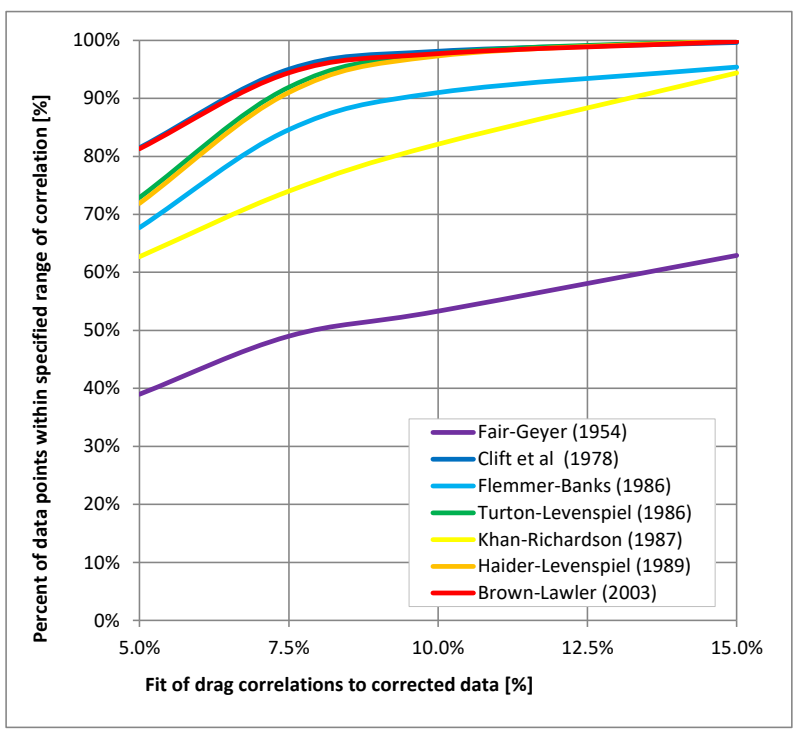

Figure S40 Range analysis method (Brown and Lawler, 2003) 


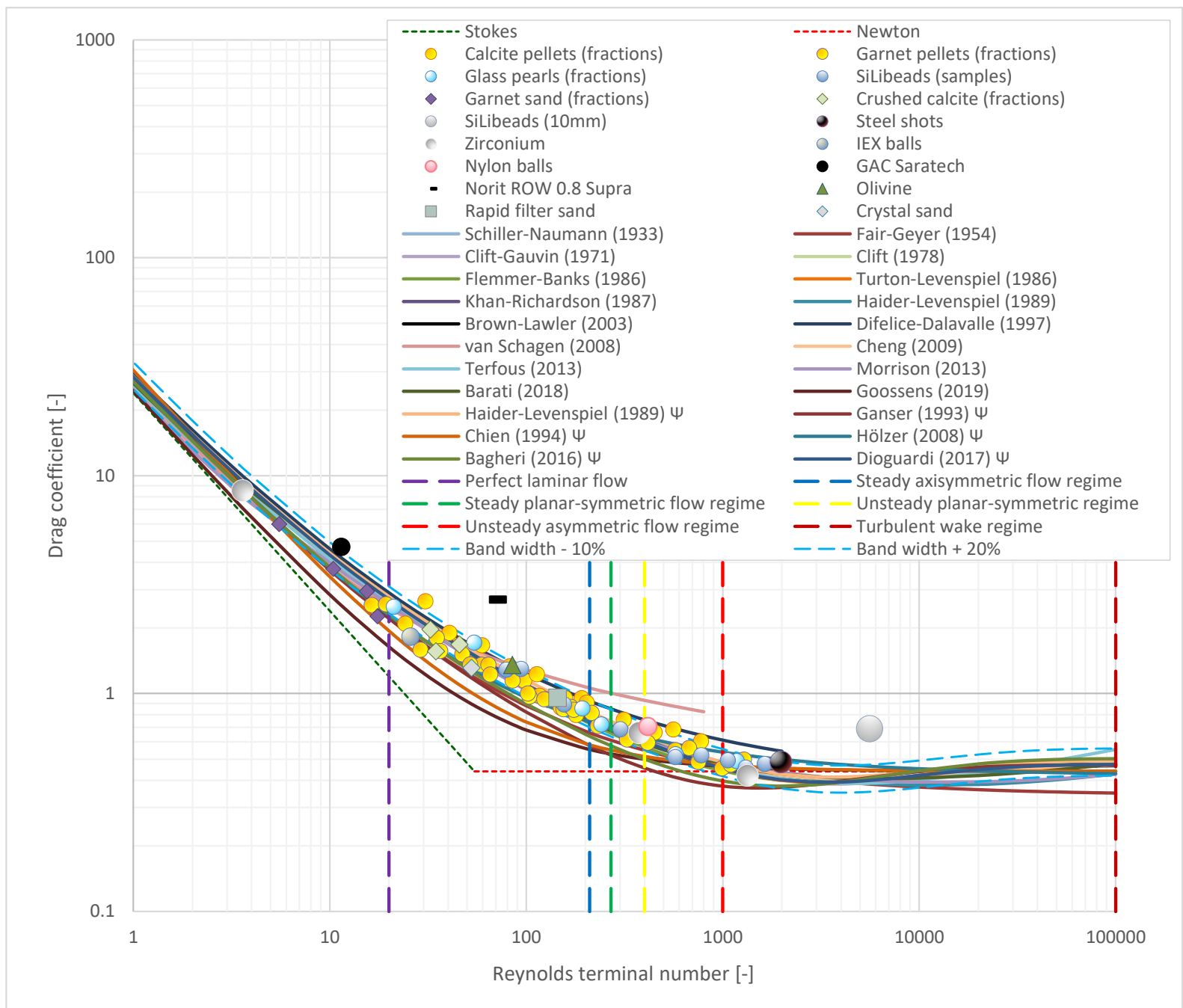

Figure S41 Standard drag curve (SDC) for 3,629 grains, using average values for 16 types of materials compared with popular prediction models for spherical and non-spherical particles. $\Phi$ indicates that the measured sphericity is included in the model.

\section{S5.7 Model prediction accuracy for data from the literature}

Data was obtained from the literature for the dimensionless drag coefficient $C_{D}$ versus the terminal Reynolds numbers $R e_{t}$

(Table S14).

Table S14 Experimental datasets from the literature

\begin{tabular}{lll}
\hline References & Shape & Data points \\
\hline (Brown and Lawler, 2003) & Spherical & 480 \\
(Wu et al., 2006) & (non)-spherical & 571 \\
(Almedeij, 2008) & Spherical & 64 \\
(Cheng, 2009) & Spherical & 43 \\
(Song et al., 2017) & (non)-spherical & 336 \\
(Breakey et al., 2018) & (non)-spherical & 2,361 \\
(Dioguardi et al., 2018) & (non)-spherical & 304 \\
\hline
\end{tabular}

Table $\mathrm{S} 13$ presents the prediction accuracy for $C_{D}$ as a function of $R e_{t}$ for exclusively spherical particles composed by (Brown and Lawler, 2003). 
Table S15 Drag coefficient prediction accuracy dataset generated by Brown and Lawler $(N=480)$. Average relative error (ARE), Normalized root mean square error (NRMSE)

\begin{tabular}{lll}
\hline Model & ARE $\left(C_{D}\right)$ & NRMSE $\left(C_{D}\right)$ \\
\hline Schiller-Naumann (1933) & $24.7 \%$ & $38.4 \%$ \\
Fair-Geyer (1954) & $10.5 \%$ & $13.0 \%$ \\
Clift-Gauvin (1971) & $4.7 \%$ & $5.9 \%$ \\
Clift (1978) & $4.3 \%$ & $5.7 \%$ \\
Turton-Levenspiel (1986) & $3.9 \%$ & $4.9 \%$ \\
Flemmer-Banks (1986) & $5.6 \%$ & $7.2 \%$ \\
Khan-Richardson (1987) & $6.2 \%$ & $7.7 \%$ \\
Difelice-Dalavalle (1997) & $12.4 \%$ & $16.5 \%$ \\
Haider-Levenspiel (1989) & $3.8 \%$ & $4.7 \%$ \\
Brown-Lawler (2003) & $3.9 \%$ & $5.1 \%$ \\
van Schagen (2008) & $17.0 \%$ & $24.7 \%$ \\
Cheng (2009) & $4.2 \%$ & $5.8 \%$ \\
Terfous (2013) & $7.2 \%$ & $15.8 \%$ \\
Morrison (2013) & $8.0 \%$ & $9.6 \%$ \\
Barati (2018) & $4.3 \%$ & $6.0 \%$ \\
Goossens (2019) & $14.1 \%$ & $18.5 \%$ \\
Haider-Levenspiel (1989) $\Phi=1$ & $5.6 \%$ & $6.6 \%$ \\
Ganser (1993) $\Phi=1$ & $10.1 \%$ & $13.2 \%$ \\
Chien (1994) $\Phi=1$ & $12.6 \%$ & $15.7 \%$ \\
\hline
\end{tabular}

Table S16 presents the prediction accuracy for $C_{D}$ as a function of $R e_{t}$ for spherical and non-spherical particles given by (Wu et al., 2006), (Almedeij, 2008), (Cheng, 2009), (Dioguardi et al., 2018), (Song et al., 2017), (Dioguardi and Mele, 2015) and (Breakey et al., 2018).

Table S16 Drag coefficient prediction accuracy for datasets from the literature $(N=3,655)$. Average relative error $(A R E)$, Normalized root mean square error (NRMSE)

\begin{tabular}{lll}
\hline Model & ARE $\left(C_{D}\right)$ & NRMSE $\left(C_{D}\right)$ \\
\hline Schiller-Naumann (1933) & $37.9 \%$ & $47.8 \%$ \\
Fair-Geyer (1954) & $37.8 \%$ & $46.0 \%$ \\
Clift-Gauvin (1971) & $32.8 \%$ & $42.4 \%$ \\
Clift (1978) & $37.1 \%$ & $47.2 \%$ \\
Turton-Levenspiel (1986) & $32.3 \%$ & $42.1 \%$ \\
Flemmer-Banks (1986) & $33.7 \%$ & $43.0 \%$ \\
Khan-Richardson (1987) & $35.1 \%$ & $43.8 \%$ \\
Difelice-Dalavalle (1997) & $28.3 \%$ & $36.5 \%$ \\
Haider-Levenspiel (1989) & $33.7 \%$ & $43.0 \%$ \\
Brown-Lawler (2003) & $33.1 \%$ & $42.6 \%$ \\
van Schagen (2008) & $27.1 \%$ & $35.3 \%$ \\
Cheng (2009) & $32.7 \%$ & $42.2 \%$ \\
Terfous (2013) 1$)$ & $34.2 \%$ & $43.1 \%$ \\
Morrison (2013) & $35.1 \%$ & $43.5 \%$ \\
Barati (2018) & $32.8 \%$ & $42.2 \%$ \\
Goossens (2019) & $43.1 \%$ & $50.8 \%$ \\
Haider-Levenspiel (1989) $\Psi$ & $30.7 \%$ & $60.5 \%$ \\
Ganser (1993) $\Psi$ & $30.4 \%$ & $58.7 \%$ \\
Chien (1994) $\Psi$ & $49.6 \%$ & $105.2 \%$ \\
1) The model by (Terfous et al., 2013) was restricted for $R e_{t}<0.01$ to avoid considerable outliers
\end{tabular}


S5.8 Comparison old-school and advanced terminal settling velocities

Table S17 Measured terminal settling velocities using old-school and advanced methods

\begin{tabular}{|c|c|c|c|c|c|c|c|c|c|}
\hline Grain types & $\begin{array}{l}d_{s, 2} \\
{[\mathrm{~mm}]}\end{array}$ & $\begin{array}{l}d_{s, 2} \\
{[\mathrm{~mm}]}\end{array}$ & $\begin{array}{l}\mathrm{T} \\
{\left[{ }^{\circ} \mathrm{C}\right]}\end{array}$ & $\begin{array}{c}v_{t, A V G} \\
{[\mathrm{~m} / \mathrm{s}]}\end{array}$ & stdev & $\begin{array}{l}\mathrm{T} \\
{\left[{ }^{\circ} \mathrm{C}\right]}\end{array}$ & $\begin{array}{l}v_{t, A V G} \\
{[\mathrm{~m} / \mathrm{s}]}\end{array}$ & stdev & $\begin{array}{l}\text { ARE } \\
{[\%]}\end{array}$ \\
\hline & \multicolumn{5}{|c|}{ Advanced } & \multicolumn{4}{|c|}{ Old-school } \\
\hline Calcite pellets & 1.00 & 1.12 & 21 & 0.15 & 0.01 & 20 & 0.14 & 0.01 & $-6 \%$ \\
\hline Calcite pellets & 1.12 & 1.25 & 21 & 0.16 & 0.01 & 20 & 0.15 & 0.01 & $-7 \%$ \\
\hline Calcite pellets & 1.25 & 1.40 & 21 & 0.18 & 0.00 & 20 & 0.17 & 0.01 & $-4 \%$ \\
\hline Calcite pellets & 1.40 & 1.70 & 21 & 0.22 & 0.01 & 23 & 0.23 & 0.01 & $4 \%$ \\
\hline Calcite pellets & 1.70 & 2.00 & 21 & 0.24 & 0.03 & 23 & 0.25 & 0.00 & $6 \%$ \\
\hline Calcite pellets & 2.00 & 2.36 & 21 & 0.27 & 0.03 & 23 & 0.28 & 0.00 & $4 \%$ \\
\hline Calcite pellets & 2.36 & 2.80 & 21 & 0.30 & 0.03 & 20 & 0.30 & 0.01 & $0 \%$ \\
\hline Calcite pellets & 2.80 & 3.35 & 21 & 0.35 & 0.01 & 19 & 0.39 & 0.03 & $12 \%$ \\
\hline Steel shots & 2.98 & 3.02 & 22 & 0.83 & n.a. ${ }^{1)}$ & $30^{2)}$ & 0.78 & 0.12 & $-6 \%$ \\
\hline Zirconium balls & 1.90 & 2.10 & 22 & 0.54 & n.a. ${ }^{1)}$ & 30 & 0.56 & 0.03 & $3 \%$ \\
\hline Glass Beads & 1.49 & 1.51 & 22 & 0.22 & n.a. ${ }^{1)}$ & 20 & 0.21 & 0.00 & $-3 \%$ \\
\hline Glass Beads & 1.98 & 2.02 & 22 & 0.28 & n.a. ${ }^{1)}$ & 20 & 0.28 & 0.28 & $1 \%$ \\
\hline Glass Beads & 2.48 & 2.52 & 22 & 0.33 & n.a. ${ }^{1)}$ & 20 & 0.32 & 0.01 & $-3 \%$ \\
\hline Glass Beads & 2.98 & 3.02 & 22 & 0.36 & n.a. ${ }^{1)}$ & 20 & 0.35 & 0.01 & $-2 \%$ \\
\hline Glass Beads & 3.47 & 3.53 & 22 & 0.41 & n.a. ${ }^{1)}$ & 20 & 0.39 & 0.02 & $-4 \%$ \\
\hline Glass Beads & 3.97 & 4.03 & 22 & 0.44 & n.a. ${ }^{1)}$ & 20 & 0.42 & 0.03 & $-5 \%$ \\
\hline
\end{tabular}

1) Only one measurement available

2) Measurements only available for $3^{\circ} \mathrm{C}$ and $32^{\circ} \mathrm{C}$ 


\section{S6 Uncertainty analysis}

A thorough uncertainty analysis (Raaghav, 2019) was performed to investigate the extent of deviation for the dimensionless drag coefficient $C_{D}$ and the terminal Reynolds number $R e_{t}$.

\section{S6.1 Basic equations}

The following basic equations were used:

The Archimedes number:

$$
\begin{aligned}
& A r=\frac{g d_{p}{ }^{3} \rho_{f}\left|\rho_{p}-\rho_{f}\right|}{\eta^{2}} \\
& A r=\frac{3}{4} C_{D} R e_{t}^{2}
\end{aligned}
$$

The Galileo number is given by:

$$
\begin{aligned}
& G a=\sqrt{A r} \\
& G a=\sqrt{\frac{g d_{p}{ }^{3} \rho_{f}\left|\rho_{p}-\rho_{f}\right|}{\eta^{2}}}
\end{aligned}
$$

The particle to fluid density ratio $\bar{\rho}$ :

$$
\bar{\rho}=\frac{\rho_{p}}{\rho_{f}}
$$

The estimate of uncertainty in $C_{D}$ and $R e_{t}$ as well as in $\rho_{p}$ and $v_{t}$ are given by the following expressions:

$$
\begin{aligned}
& C_{D}=\frac{4}{3} \frac{g d_{p}\left|\rho_{p}-\rho_{f}\right|}{v_{t}^{2} \rho_{f}} \\
& R e_{t}=\frac{\rho_{f} d_{p} v_{t}}{\eta}
\end{aligned}
$$

Rewriting:

$$
R e_{t}=\frac{d_{p} v_{t}}{v_{T}}
$$

Result: 
$C_{D}=\frac{\frac{4}{3} g d_{p} \rho_{p}}{v_{t}^{2} \rho_{f}}-\frac{\frac{4}{3} g d_{p}}{v_{t}^{2}}$

\section{S6.2 Overview uncertainty analysis equations and contribution to error}

Table S18 Uncertainty analysis equations

\begin{tabular}{|c|c|c|}
\hline Variable & Equation & Eq. nr. \\
\hline Terminal Reynolds number & $\delta R e_{t}=\sqrt{\left(\frac{\partial R e_{t}}{\partial d_{p}} \delta d_{p}\right)^{2}+\left(\frac{\partial R e_{t}}{\partial v_{T}} \delta v_{T}\right)^{2}+\left(\frac{\partial R e_{t}}{\partial v_{t}} \delta v_{t}\right)^{2}}$ & (69) \\
\hline Drag coefficient & $\delta C_{D}=\sqrt{\left(\frac{\partial C_{D}}{\partial g} \delta g\right)^{2}+\left(\frac{\partial C_{D}}{\partial d_{p}} \delta d_{p}\right)^{2}+\left(\frac{\partial C_{D}}{\partial \rho_{p}} \delta \rho_{p}\right)^{2}+\left(\frac{\partial C_{D}}{\partial \rho_{f}} \delta \rho_{f}\right)^{2}+\left(\frac{\partial C_{D}}{\partial v_{t}} \delta v_{t}\right)^{2}}$ & $(70)$ \\
\hline Particle density & $\delta \rho_{p}=\sqrt{\left(\frac{\partial \rho_{p}}{\partial m_{p}} \delta m_{p}\right)^{2}+\left(\frac{\partial \rho_{p}}{\partial d_{p}} \delta d_{p}\right)^{2}}$ & $(71)$ \\
\hline Terminal settling velocity ${ }^{1)}$ & $\delta v_{t}=\sqrt{\left(\frac{\partial v_{t}}{\partial L} \delta L\right)^{2}+\left(\frac{\partial v_{t}}{\partial t} \delta t\right)^{2}}$ & $(72)$ \\
\hline
\end{tabular}

1) Human response time inaccuracy correction is given in $\S \mathrm{S} 6.9 .4$

Table S19 Uncertainty analysis equations

\begin{tabular}{|c|c|c|c|}
\hline Variable & Term & Equation & $\begin{array}{l}\text { Eq. nr. } \\
\text { (contribution to error) }\end{array}$ \\
\hline \multirow[t]{3}{*}{ Terminal Reynolds number (Eq. 67) } & $1^{\text {st }}$ & $\frac{\partial R e_{t}}{\partial d_{p}}=\frac{v_{t}}{v_{T}}$ & (73) \\
\hline & $2^{\text {nd }}$ & $\frac{\partial R e_{t}}{\partial T}=\frac{d_{p} v_{t}}{c_{6}}\left(-\frac{c_{7} \ln 10(1+\alpha \Delta T)}{\left(T+c_{8}\right)^{2} 10^{c_{7} /\left(T+c_{8}\right)}}-\frac{\alpha}{10^{c_{7} /\left(T+c_{8}\right)}}\right)$ & $(74)$ \\
\hline & $3^{\text {rd }}$ & $\frac{\partial R e_{t}}{\partial v_{t}}=\frac{1}{v_{T}}\left(d_{p}-\frac{c_{2} d_{p}^{2}}{D}\right)$ & $(75)$ \\
\hline \multirow[t]{5}{*}{ Drag coefficient (Eq. 68) } & $1^{\text {st }}$ & $\frac{\partial C_{D}}{\partial g}=\frac{4}{3} \frac{d_{p}}{v_{t}^{2}}\left(\frac{\rho_{p}}{\rho_{f}}-1\right)$ & $(76)$ \\
\hline & $2^{\text {nd }}$ & $\frac{\partial C_{D}}{\partial d_{p}}=\frac{\frac{4}{3} \frac{g}{v_{t}^{2}}\left(\frac{\rho_{p}}{\rho_{f}}-1\right)}{\left(d_{n^{\frac{1}{2}}}\right)^{3}}\left(d_{p^{-\frac{3}{2}}}+\frac{c_{2}}{D} d_{p^{-\frac{1}{2}}}\right)$ & $(77)$ \\
\hline & $3^{\text {rd }}$ & $\frac{\partial C_{D}}{\partial \rho_{p}}=\frac{4}{3} \frac{g d_{p}}{v_{t}^{2} \rho_{f}}$ & (78) \\
\hline & $4^{\text {th }}$ & $\frac{\partial C_{D}}{\partial T}=\frac{4}{3} \frac{g d_{p}}{v_{t}^{2}}\left(\rho_{p}\left(\frac{c_{3} e^{c_{3} T}}{c_{4}-c_{5} T^{2}}+\frac{2 c_{5} T e^{c_{3} T}}{\left(c_{4}-c_{5} T^{2}\right)^{2}}\right)-c_{3} e^{c_{3} T}\right)$ & $(79)$ \\
\hline & $5^{\text {th }}$ & $\frac{\partial C_{D}}{\partial v_{t}}=-\frac{8}{3} \frac{g d_{p}}{v_{t}^{3}} \frac{\left(\frac{\rho_{p}}{\rho_{f}}-1\right)}{\left(1-c_{2} \frac{d_{p}}{D}\right)^{2}}$ & $(80)$ \\
\hline \multirow[t]{2}{*}{ Particle density (Eq. 71) } & $1^{\mathrm{st}}$ & $\frac{\partial \rho_{p}}{\partial m_{p}}=\frac{6}{\pi d_{p}^{3}}$ & $(81)$ \\
\hline & $2^{\text {nd }}$ & $\frac{\partial \rho_{p}}{\partial d_{p}}=-\frac{18 m_{p}}{\pi d_{p}^{4}}$ & $(82)$ \\
\hline \multirow[t]{2}{*}{ Terminal settling velocity (Eq. 72) } & $1^{\mathrm{st}}$ & $\frac{\partial v_{t}}{\partial L}=\frac{1}{t}$ & $(83)$ \\
\hline & $2^{\text {nd }}$ & $\frac{\partial v_{t}}{\partial t}=-\frac{L}{t^{2}}$ & $(84)$ \\
\hline
\end{tabular}




\section{S6.3 Simplifications}

\section{S6.3.1 Archimedes and Galileo numbers}

$$
\begin{aligned}
& A r=\frac{g d_{p}{ }^{3}|\bar{\rho}-1|}{v_{T}{ }^{2}} \\
& G a=\sqrt{\frac{|\bar{\rho}-1| g d_{p}{ }^{3}}{v_{T}{ }^{2}}}
\end{aligned}
$$

\section{S6.3.2 Linear thermal expansion}

To take temperature variations into account, a linear heat or thermal expansion equation is used (Perry and Green, 2007); (Rumble, 2019); (Serway and Jewett, 2014) where the particle diameter is proportional to the original length, the change in temperature and its linear heat expansion coefficient, expressed as:

$$
\begin{aligned}
& d_{p, 1}=d_{p, 0}(1+\alpha \Delta T) \\
& \Delta T=T_{0}-T \\
& d_{p, 1}=d_{p, 0}+\alpha T_{0} d_{p, 0}-\alpha T d_{p, 0} \\
& d_{p, 1}=d_{0}\left(1+\alpha T_{0}-\alpha T\right)
\end{aligned}
$$

Table S21 presents linear heat expansion coefficients.

To find analytical derivatives, a simplified empirical equation is proposed.

$$
\begin{aligned}
& \frac{d_{p, 1}}{d_{p, 0}}=c_{4} e^{c_{5} T} \\
& d_{p, 1}=d_{p, 0} c_{4} e^{c_{5} T}
\end{aligned}
$$

Fit parameters were obtained through non-linear fitting (Koza, 1992); (Nutonian, 2019). Table S20 includes fit parameters. 


\section{S6.3.3 Kinematic viscosity}

A direct temperature dependency can be expressed through the following empirical Equation (Sbased on the Vogel-FulcherTammann relation):

$$
v_{T}=c_{6} 10^{c_{7} /\left(T+c_{8}\right)}
$$

Table S20 includes parameters.

\section{S6.4 Drag Reynolds relationships}

The dimensionless drag coefficient $C_{D}$ and the terminal Reynolds number $R e_{t}$ are given by:

$$
\begin{aligned}
& C_{D}=\frac{4}{3} \frac{g d_{p}\left|\rho_{p}-\rho_{f}\right|}{v_{t}^{2} \rho_{f}} \\
& R e_{t}=\frac{\rho_{f} d_{p} v_{t}}{\eta}
\end{aligned}
$$

Where:

$C_{D}$ is a function of $g, d_{p}, \rho_{p}, \rho_{f}, v_{t}$.

$R e_{t}$ is a function of $d_{p}, \rho_{f}, v_{t}, \eta$.

Direct measurements: particle properties: $d_{p}, \rho_{p}, \psi$

$$
\begin{aligned}
& \text { fluid properties: } \rho_{f}, T \\
& \text { experimental: } g, D, L, t
\end{aligned}
$$

Rewriting:

$$
R e_{t}=\frac{d_{p} v_{t}}{v_{T}}
$$

Result:

$$
C_{D}=\frac{\frac{4}{3} g d_{p} \rho_{p}}{v_{t}^{2} \rho_{f}}-\frac{\frac{4}{3} g d_{p}}{v_{t}^{2}}
$$




\section{S6.5 Uncertainty expressions}

The estimate of uncertainty in $C_{D}$ and $R e_{t}$ as well as in $\rho_{p}$ and $v_{t}$ are given by the following expressions:

$$
\begin{aligned}
& \delta R e_{t}=\sqrt{\left(\frac{\partial R e_{t}}{\partial d_{p}} \delta d_{p}\right)^{2}+\left(\frac{\partial R e_{t}}{\partial v_{T}} \delta v_{T}\right)^{2}+\left(\frac{\partial R e_{t}}{\partial v_{t}} \delta v_{t}\right)^{2}} \\
& \delta C_{D}=\sqrt{\left(\frac{\partial C_{D}}{\partial g} \delta g\right)^{2}+\left(\frac{\partial C_{D}}{\partial d_{p}} \delta d_{p}\right)^{2}+\left(\frac{\partial C_{D}}{\partial \rho_{p}} \delta \rho_{p}\right)^{2}+\left(\frac{\partial C_{D}}{\partial \rho_{f}} \delta \rho_{f}\right)^{2}+\left(\frac{\partial C_{D}}{\partial v_{t}} \delta v_{t}\right)^{2}} \\
& \delta \rho_{p}=\sqrt{\left(\frac{\partial \rho_{p}}{\partial m_{p}} \delta m_{p}\right)^{2}+\left(\frac{\partial \rho_{p}}{\partial d_{p}} \delta d_{p}\right)^{2}} \\
& \delta v_{t}=\sqrt{\left(\frac{\partial v_{t}}{\partial L} \delta L\right)^{2}+\left(\frac{\partial v_{t}}{\partial t} \delta t\right)^{2}}
\end{aligned}
$$

\section{S6.6 Reynolds uncertainty}

Accordingly, the error (uncertainty $\delta$ ) concerning the terminal Reynolds number velocity $R e_{t}$ can be determined (Ku, 1966):

$$
\delta R e_{t}=\sqrt{\left(\frac{\partial R e_{t}}{\partial d_{p}} \delta d_{p}\right)^{2}+\left(\frac{\partial R e_{t}}{\partial v_{T}} \delta v_{T}\right)^{2}+\left(\frac{\partial R e_{t}}{\partial v_{t}} \delta v_{t}\right)^{2}}
$$

The kinematic viscosity converted into temperature dependency:

$$
\delta R e_{t}=\sqrt{\left(\frac{\partial R e_{t}}{\partial d_{p}} \delta d_{p}\right)^{2}+\left(\frac{\partial R e_{t}}{\partial v_{T}} \frac{\partial v_{T}}{\partial T} \delta T\right)^{2}+\left(\frac{\partial R e_{t}}{\partial v_{t}} \delta v_{t}\right)^{2}}
$$

\section{S6.6.1 First term}

$$
\begin{gathered}
\frac{\partial R e_{t}}{\partial d_{p}}=\frac{\partial\left(\frac{d_{p} v_{t}}{v_{T}}\right)}{\partial d_{p}} \\
\frac{\partial R e_{t}}{\partial d_{p}}=\frac{v_{t}}{v_{T}} \frac{\partial\left(d_{p}\right)}{\partial d_{p}}
\end{gathered}
$$

Result: 


$$
\frac{\partial R e_{t}}{\partial d_{p}}=\frac{v_{t}}{v_{T}}
$$

Also conserving the shape factor:

$$
d_{p}=d_{p, 0} \Phi
$$

$\frac{\partial R e_{t}}{\partial d_{p}}=\frac{v_{t}}{v_{T}} \Phi \frac{\partial\left(d_{p}\right)}{\partial d_{p}}$

Result:

$$
\frac{\partial R e_{t}}{\partial d_{p}}=\frac{v_{t}}{v_{T}} \Phi
$$

\section{S6.6.2 Second term}

\subsubsection{No heat expansion correction (viscosity dependency)}

$$
\begin{aligned}
& \frac{\partial R e_{t}}{\partial v_{T}}=\frac{\partial\left(\frac{d_{p} v_{t}}{v_{T}}\right)}{\partial v_{T}} \\
& \frac{\partial R e_{t}}{\partial v_{T}}=d_{p} v_{t} \frac{\partial\left(\frac{1}{v_{T}}\right)}{\partial v_{T}}
\end{aligned}
$$

Result:

$$
\frac{\partial R e_{t}}{\partial v_{T}}=-\frac{d_{p} v_{t}}{v_{T}^{2}}
$$

6.6.2.2 No heat expansion correction (temperature dependency)

$$
\frac{\partial R e_{t}}{\partial T}=\frac{\partial\left(\frac{d_{p} v_{t}}{v_{T}}\right)}{\partial T}
$$

A simplified empirical equation for the kinematic viscosity:

$$
v_{T}=c_{0} e^{c_{1} T}
$$


Table S20 includes parameters.

$\frac{\partial R e_{t}}{\partial T}=\frac{\partial\left(\frac{d_{p} v_{t}}{c_{0} e^{c_{1} T}}\right)}{\partial T}$
$\frac{\partial R e_{t}}{\partial T}=\frac{d_{p} v_{t}}{c_{0}} \frac{\partial\left(e^{-c_{1} T}\right)}{\partial T}$

Result:

$\frac{\partial R e_{t}}{\partial T}=-\frac{c_{1}}{c_{0}} d_{p} v_{t} e^{-c_{1} T}$

6.6.2.3 With heat expansion correction (alternative)

$$
\begin{aligned}
& \frac{\partial R e_{t}}{\partial T}=\frac{\partial\left(\frac{d_{p} v_{t}}{v_{T}}\right)}{\partial T} \\
& v_{T}=c_{0} e^{c_{1} T} \\
& d_{p, 1}=d_{p, 0} e^{c_{3} T}
\end{aligned}
$$

Table S20 includes parameters.

$$
\begin{aligned}
& \frac{\partial R e_{t}}{\partial T}=v_{t} \frac{\partial\left(\frac{d_{p, 0} e^{c_{3} T}}{c_{0} e^{c_{1} T}}\right)}{\partial T} \\
& \frac{\partial R e_{t}}{\partial T}=\frac{v_{t} d_{p}}{c_{0}} \frac{\partial\left(e^{\left(c_{3}-c_{1}\right) T}\right)}{\partial T}
\end{aligned}
$$

Result:

$$
\frac{\partial R e_{t}}{\partial T}=\frac{\left(c_{3}-c_{1}\right)}{c_{0}} v_{t} d_{p} e^{\left(c_{3}-c_{1}\right) T}
$$

Assumed variation in temperature $1{ }^{\circ} \mathrm{C}$. 
$c_{3}=0$

$\frac{\partial R e_{t}}{\partial T}=-\frac{c_{1}}{c_{0}} v_{t} d_{p} e^{-c_{1} T}$

Check: OK.

\subsubsection{With heat expansion correction (original)}

To take heat expansion into account, equation 87 is used; to take temperature dependency into account, equation 93 is used.

Table S20 includes parameters. Plugging in the equations leads to the following:

$\frac{\partial R e_{t}}{\partial T}=\frac{\partial\left(\frac{d_{p} v_{t}}{v_{T}}\right)}{\partial T}$

$\frac{\partial \operatorname{Re}_{t}}{\partial T}=\frac{\partial\left(\frac{\left(d_{0}\left(1+\alpha T_{0}-\alpha T\right)\right) v_{t}}{c_{6} 10^{c_{7} /\left(T+c_{8}\right)}}\right)}{\partial T}$

$\frac{\partial R e_{t}}{\partial T}=\frac{d_{0} v_{t}}{c_{6}} \frac{\partial\left(\frac{1+\alpha T_{0}-\alpha T}{10^{c_{7} /\left(T+c_{8}\right)}}\right)}{\partial T}$

Result:

$\frac{\partial R e_{t}}{\partial T}=\frac{d_{p} v_{t}}{c_{6}}\left(-\frac{c_{7} \ln 10(1+\alpha \Delta T)}{\left(T+c_{8}\right)^{2} 10^{c_{7} /\left(T+c_{8}\right)}}-\frac{\alpha}{10^{c_{7} /\left(T+c_{8}\right)}}\right)$

\section{S6.6.3 Third term}

\subsubsection{No wall effects correction}

$$
\begin{aligned}
& \frac{\partial R e_{t}}{\partial v_{t}}=\frac{\partial\left(\frac{d_{p} v_{t}}{v_{T}}\right)}{\partial v_{t}} \\
& \frac{\partial R e_{t}}{\partial v_{t}}=\frac{d_{p}}{v_{T}} \frac{\partial\left(v_{t}\right)}{\partial v_{t}}
\end{aligned}
$$

Result: 


$$
\frac{\partial R e_{t}}{\partial v_{t}}=\frac{d_{p}}{v_{T}}
$$

\subsubsection{Wall effects correction}

Based on the simplified Ladenburg wall effects correction Equation (SEquation (S13), the retardation of the motion of individual particles settling in a cylinder due to the displacement and opposing motion of the surrounding fluid states is expressed as follows:

$$
v_{t}=\left(1-c_{2} \lambda\right) v_{t, \infty}
$$

where

$$
\lambda=\frac{d_{p}}{D}
$$

Table S20 includes parameters.

$$
\begin{aligned}
& v_{t}=\left(1-c_{2}\left(\frac{d_{p}}{D}\right)\right) v_{t, \infty} \\
& v_{t}=\left(v_{t}-c_{2} \frac{d_{p} v_{t}}{D}\right) \\
& \frac{\partial e_{t}}{\partial v_{t}}=\frac{1}{v_{T}} \frac{\partial\left(v_{t} d_{p}\right)}{\partial v_{t}}
\end{aligned}
$$

Rewriting:

$$
\begin{aligned}
& \frac{\partial R e_{t}}{\partial v_{t}}=\frac{1}{v_{T}} \frac{\partial\left(\left(v_{t}-c_{2} \frac{d_{p} v_{t}}{D}\right) d_{p}\right)}{\partial v_{t}} \\
& \frac{\partial R e_{t}}{\partial v_{t}}=\frac{1}{v_{T}} \frac{\partial\left(d_{p} v_{t}-c_{2} \frac{d_{p}{ }^{2} v_{t}}{D}\right)}{\partial v_{t}} \\
& \frac{\partial R e_{t}}{\partial v_{t}}=\frac{1}{v_{T}}\left(\left(d_{p}-\frac{c_{2} d_{p}{ }^{2}}{D}\right) \frac{\partial\left(v_{t}\right)}{\partial v_{t}}\right)
\end{aligned}
$$

Result: 


$$
\frac{\partial R e_{t}}{\partial v_{t}}=\frac{1}{v_{T}}\left(d_{p}-\frac{c_{2} d_{p}^{2}}{D}\right)
$$

\subsubsection{Check: no wall effects}

$$
\begin{aligned}
& \frac{\partial R e_{t}}{\partial v_{t}}=\frac{1}{v_{T}}\left(d_{p}-\frac{c_{2} d_{p}{ }^{2}}{D}\right) \\
& \frac{\partial R e_{t}}{\partial v_{t}}=\frac{d_{p}}{v_{T}}\left(1-c_{2} \frac{d_{p}}{D}\right) \\
& \frac{v_{t}}{v_{t, \infty}}=\left(1-c_{2} \frac{d_{p}}{D}\right)=1 \\
& \frac{\partial R e_{t}}{\partial v_{t}}=\frac{d_{p}}{v_{T}}
\end{aligned}
$$

Check: OK.

\section{S6.7 Drag uncertainty}

Accordingly, the error (uncertainty $\delta$ ) considering the drag coefficient $C_{D}$ can be determined:

$$
\begin{aligned}
& \delta C_{D} \\
& =\sqrt{\left(\frac{\partial C_{D}}{\partial g} \delta g\right)^{2}+\left(\frac{\partial C_{D}}{\partial d_{p}} \delta d_{p}\right)^{2}+\left(\frac{\partial C_{D}}{\partial \rho_{p}} \delta \rho_{p}\right)^{2}+\left(\frac{\partial C_{D}}{\partial \rho_{f}} \delta \rho_{f}\right)^{2}+\left(\frac{\partial C_{D}}{\partial v_{t}} \delta v_{t}\right)^{2}}
\end{aligned}
$$

The fluid density dependency is adjusted for a direct temperature dependency according to the following expression:

$$
\delta C_{D}=\sqrt{\left(\frac{\partial C_{D}}{\partial g} \delta g\right)^{2}+\left(\frac{\partial C_{D}}{\partial d_{p}} \delta d_{p}\right)^{2}+\left(\frac{\partial C_{D}}{\partial \rho_{p}} \delta \rho_{p}\right)^{2}+\left(\frac{\partial C_{D}}{\partial \rho_{f}} \frac{\partial \rho_{f}}{\partial T} \delta T\right)^{2}+\left(\frac{\partial C_{D}}{\partial v_{t}} \delta v_{t}\right)}
$$

\section{S6.7.1 First term}

$$
\frac{\partial C_{D}}{\partial g}=\frac{\partial\left(\frac{\frac{4}{3} g d_{p} \rho_{p}}{v_{t}^{2} \rho_{f}}-\frac{\frac{4}{3} g d_{p}}{v_{t}^{2}}\right)}{\partial g}
$$




$$
\begin{aligned}
& \frac{\partial C_{D}}{\partial g}=\left(\frac{\frac{4}{3} d_{p} \rho_{p}}{v_{t}^{2} \rho_{f}}-\frac{\frac{4}{3} d_{p}}{v_{t}^{2}}\right) \frac{\partial(g)}{\partial g} \\
& \frac{\partial C_{D}}{\partial g}=\frac{4}{3} \frac{d_{p}}{v_{t}^{2}}\left(\frac{\rho_{p}}{\rho_{f}}-1\right) \frac{\partial(g)}{\partial g}
\end{aligned}
$$

Result:

$$
\frac{\partial C_{D}}{\partial g}=\frac{4}{3} \frac{d_{p}}{v_{t}^{2}}\left(\frac{\rho_{p}}{\rho_{f}}-1\right)
$$

\section{S6.7.2 Second term}

\subsubsection{No wall effects correction}

$$
\begin{aligned}
& \frac{\partial C_{D}}{\partial d_{p}}=\left(\frac{\frac{4}{3} g \rho_{p}}{v_{t}^{2} \rho_{f}}-\frac{\frac{4}{3} g}{v_{t}{ }^{2}}\right) \frac{\partial\left(d_{p}\right)}{\partial d_{p}} \\
& \frac{\partial C_{D}}{\partial d_{p}}=\left(\frac{\frac{4}{3} g \rho_{p}}{v_{t}^{2} \rho_{f}}-\frac{\frac{4}{3} g}{v_{t}^{2}}\right)
\end{aligned}
$$

Result:

$$
\frac{\partial C_{D}}{\partial d_{p}}=\frac{4}{3} \frac{g}{v_{t}^{2}}\left(\frac{\rho_{p}}{\rho_{f}}-1\right)
$$

\subsubsection{Wall effects correction}

$$
\begin{aligned}
& \frac{\partial C_{D}}{\partial d_{p}}=\frac{4}{3} g\left(\frac{\rho_{p}}{\rho_{f}}-1\right) \frac{\partial\left(\frac{d_{p}}{v_{t}^{2}}\right)}{\partial d_{p}} \\
& \frac{v_{t}}{v_{t, \infty}}=1-c_{2}\left(\frac{d_{p}}{D}\right) \\
& v_{t}=\left(v_{t}-c_{2} \frac{d_{p} v_{t}}{D}\right)
\end{aligned}
$$


$\frac{\partial C_{D}}{\partial d_{p}}=\frac{4}{3} g\left(\frac{\rho_{p}}{\rho_{f}}-1\right) \frac{\partial\left(\frac{d_{p}}{\left(v_{t}-c_{2} \frac{d_{p} v_{t}}{D}\right)^{2}}\right)}{\partial d_{p}}$

$\frac{\partial C_{D}}{\partial d_{p}}=\frac{4}{3} g\left(\frac{\rho_{p}}{\rho_{f}}-1\right) \frac{\partial\left(d_{p}\left(v_{t}-c_{2} \frac{d_{p} v_{t}}{D}\right)^{-2}\right)}{\partial d_{p}}$

$\frac{\partial C_{D}}{\partial d_{p}}=\frac{4}{3} g\left(\frac{\rho_{p}}{\rho_{f}}-1\right) \frac{\partial\left(\left(d_{p}^{-\frac{1}{2}} v_{t}-d_{p}^{-\frac{1}{2}} C_{2} \frac{d_{p} v_{t}}{D}\right)^{-2}\right)}{\partial d_{p}}$

$\frac{\partial C_{D}}{\partial d_{p}}=\frac{4}{3} g\left(\frac{\rho_{p}}{\rho_{f}}-1\right) \frac{\partial\left(\frac{d_{p}}{v_{t}^{2}}\right)}{\partial d_{p}}$

$v_{t}=\left(v_{t}-c_{2} \frac{d_{p} v_{t}}{D}\right)$

$\frac{\partial C_{D}}{\partial d_{p}}=\frac{4}{3} g\left(\frac{\rho_{p}}{\rho_{f}}-1\right) \frac{\partial\left(\frac{d_{p}}{\left(v_{t}-c_{2} \frac{d_{p} v_{t}}{D}\right)^{2}}\right)}{\partial d_{p}}$

$\frac{\partial C_{D}}{\partial d_{p}}=\frac{4}{3} g\left(\frac{\rho_{p}}{\rho_{f}}-1\right) \frac{\partial\left(d_{p}\left(v_{t}-c_{2} \frac{d_{p} v_{t}}{D}\right)^{-2}\right)}{\partial d_{p}}$

$\frac{\partial C_{D}}{\partial d_{p}}=\frac{4}{3} g\left(\frac{\rho_{p}}{\rho_{f}}-1\right) \frac{\partial\left(\left(d_{p}{ }^{-\frac{1}{2}} v_{t}-d_{p}{ }^{-\frac{1}{2}} c_{2} \frac{d_{p} v_{t}}{D}\right)^{-2}\right)}{\partial d_{p}}$

$\frac{\partial C_{D}}{\partial d_{p}}=\frac{4}{3} \frac{g}{v_{t}^{2}}\left(\frac{\rho_{p}}{\rho_{f}}-1\right) \frac{\partial\left(\left(d_{p}{ }^{-\frac{1}{2}}-c_{2} \frac{d_{p^{\frac{1}{2}}}}{D}\right)^{-2}\right)}{\partial d_{p}}$ 


$$
\begin{aligned}
& \frac{\partial C_{D}}{\partial d_{p}}=-2 \frac{\frac{4}{3} \frac{g}{v_{t}^{2}}\left(\frac{\rho_{p}}{\rho_{f}}-1\right)}{\left(d_{p}{ }^{-\frac{1}{2}}-c_{2} \frac{d_{p}^{\frac{1}{2}}}{D}\right)^{3}} \frac{\partial\left(d_{p}^{-\frac{1}{2}}-c_{2} \frac{d_{p^{\frac{1}{2}}}}{D}\right)}{\partial d_{p}} \\
& \frac{\partial C_{D}}{\partial d_{p}}=-2 \frac{\frac{4}{3} \frac{g}{v_{t}^{2}}\left(\frac{\rho_{p}}{\rho_{f}}-1\right)}{\left(d_{p}^{-\frac{1}{2}}-c_{2} \frac{d_{p}^{\frac{1}{2}}}{D}\right)^{3}}\left(\frac{\partial\left(d_{p}^{-\frac{1}{2}}\right)}{\partial d_{p}}-\frac{c_{2}}{D} \frac{\partial\left(d_{p} \frac{1}{2}\right)}{\partial d_{p}}\right)
\end{aligned}
$$

Result:

$$
\frac{\partial C_{D}}{\partial d_{p}}=\frac{\frac{4}{3} \frac{g}{v_{t}^{2}}\left(\frac{\rho_{p}}{\rho_{f}}-1\right)}{\left(d_{p}^{-\frac{1}{2}}-c_{2} \frac{d_{p^{2}}}{D}\right)^{3}}\left(d_{p}^{-\frac{3}{2}}+\frac{c_{2}}{D} d_{p}^{-\frac{1}{2}}\right)
$$

\subsubsection{Check: no wall effects}

$$
\begin{aligned}
& \frac{v_{t}}{v_{t, \infty}}=1-c_{2}\left(\frac{d_{p}}{D}\right) \\
& c_{2}=0
\end{aligned}
$$

$\frac{\partial C_{D}}{\partial d_{p}}=\frac{\frac{4}{3} \frac{g}{v_{t}^{2}}\left(\frac{\rho_{p}}{\rho_{f}}-1\right)}{\left(d_{p}^{-\frac{1}{2}}\right)^{3}}\left(d_{p}{ }^{-\frac{3}{2}}\right)$

$$
\frac{\partial C_{D}}{\partial d_{p}}=\frac{4}{3} \frac{g}{v_{t}^{2}}\left(\frac{\rho_{p}}{\rho_{f}}-1\right)
$$

Check: OK.

\section{S6.7.3 Third term}

$$
\frac{\partial C_{D}}{\partial \rho_{p}}=\frac{\partial\left(\frac{\frac{4}{3} g d_{p} \rho_{p}}{v_{t}^{2} \rho_{f}}-\frac{\frac{4}{3} g d_{p}}{v_{t}^{2}}\right)}{\partial \rho_{p}}
$$


$\frac{\partial C_{D}}{\partial \rho_{p}}=\frac{\partial\left(\frac{\frac{4}{3} g d_{p} \rho_{p}}{v_{t}^{2} \rho_{f}}\right)}{\partial \rho_{p}}$

$\frac{\partial C_{D}}{\partial \rho_{p}}=\frac{\frac{4}{3} g d_{p}}{v_{t}^{2} \rho_{f}} \frac{\partial\left(\rho_{p}\right)}{\partial \rho_{p}}$

Result:

$\frac{\partial C_{D}}{\partial \rho_{p}}=\frac{4}{3} \frac{g d_{p}}{v_{t}^{2} \rho_{f}}$

\section{S6.7.4 Fourth term}

\subsubsection{Density temperature correction}

$\frac{\partial C_{D}}{\partial \rho_{f}}=\frac{\partial\left(\frac{\frac{4}{3} g d_{p} \rho_{p}}{v_{t}^{2} \rho_{f}}-\frac{\frac{4}{3} g d_{p}}{v_{t}^{2}}\right)}{\partial \rho_{f}}$

$\frac{\partial C_{D}}{\partial \rho_{f}}=\frac{\frac{4}{3} g d_{p} \rho_{p}}{v_{t}^{2}} \frac{\partial\left(\frac{1}{\rho_{f}}\right)}{\partial \rho_{f}}$

$\frac{\partial C_{D}}{\partial \rho_{f}}=\frac{-\frac{4}{3} g d_{p} \rho_{p}}{v_{t}^{2}} \frac{1}{\rho_{f}^{2}}$

Result:

$\frac{\partial C_{D}}{\partial \rho_{f}}=-\frac{4}{3} \frac{g d_{p} \rho_{p}}{v_{t}^{2} \rho_{f}^{2}}$

6.7.4.2 With heat expansion correction

$$
\begin{gathered}
\frac{\partial C_{D}}{\partial \rho_{f}}=\frac{\partial\left(\frac{\frac{4}{3} g d_{p} \rho_{p}}{v_{t}^{2} \rho_{f}}-\frac{\frac{4}{3} g d_{p}}{v_{t}^{2}}\right)}{\partial \rho_{f}} \\
\frac{\partial C_{D}}{\partial T}=\frac{\partial\left(\frac{\frac{4}{3} g d_{p} \rho_{p}}{v_{t}^{2} \rho_{f}}-\frac{4}{\frac{3}{2} g d_{p}} v_{t}^{2}\right)}{\partial T}
\end{gathered}
$$


$\frac{\partial C_{D}}{\partial T}=\frac{4}{3} \frac{g}{v_{t}^{2}} \frac{\partial\left(\frac{d_{p} \rho_{p}}{\rho_{f}}-d_{p}\right)}{\partial T}$

$\frac{\partial C_{D}}{\partial T}=\frac{4}{3} \frac{g}{v_{t}^{2}}\left(\rho_{p} \frac{\partial\left(\frac{d_{p}}{\rho_{f}}\right)}{\partial T}-\frac{\partial\left(d_{p}\right)}{\partial T}\right)$

$\rho_{f}=c_{4}-c_{5} T^{2}$

$d_{p, 1}=d_{p, 0} e^{c_{3} T}$

$\frac{\partial C_{D}}{\partial T}=\frac{4}{3} \frac{g d_{p}}{v_{t}{ }^{2}}\left(\rho_{p} \frac{\partial\left(\frac{e^{C_{3} T}}{\rho_{f}}\right)}{\partial T}-\frac{\partial\left(e^{c_{3} T}\right)}{\partial T}\right)$

$\frac{\partial C_{D}}{\partial T}=\frac{4}{3} \frac{g d_{p}}{v_{t}^{2}}\left(\rho_{p} \frac{\partial\left(\frac{e^{c_{3} T}}{c_{4}-C_{5} T^{2}}\right)}{\partial T}-\frac{\partial\left(e^{c_{3} T}\right)}{\partial T}\right)$

Result:

$\frac{\partial C_{D}}{\partial T}=\frac{4}{3} \frac{g d_{p}}{v_{t}^{2}}\left(\rho_{p}\left(\frac{c_{3} e^{c_{3} T}}{c_{4}-c_{5} T^{2}}+\frac{2 c_{5} T e^{c_{3} T}}{\left(c_{4}-c_{5} T^{2}\right)^{2}}\right)-c_{3} e^{c_{3} T}\right)$

\subsubsection{Check: density constant}

$\frac{\partial C_{D}}{\partial T}=\frac{4}{3} \frac{g}{v_{t}^{2}} \frac{\partial\left(\frac{d_{p} \rho_{p}}{\rho_{f}}-d_{p}\right)}{\partial T}$

$\frac{\partial C_{D}}{\partial T}=\frac{4}{3} \frac{g}{v_{t}^{2}} \frac{\partial\left(\left(\frac{\rho_{p}}{\rho_{f}}-1\right) d_{p}\right)}{\partial T}$

$\frac{\partial C_{D}}{\partial T}=\frac{4}{3} \frac{g}{v_{t}^{2}}\left(\frac{\rho_{p}}{\rho_{f}}-1\right) \frac{\partial d_{p}}{\partial T}$

$\frac{\partial C_{D}}{\partial T}=\frac{4}{3} \frac{g d_{p}}{v_{t}^{2}}\left(\frac{\rho_{p}}{\rho_{f}}-1\right) \frac{\partial e^{c_{3} T}}{\partial T}$ 
$\frac{\partial C_{D}}{\partial T}=\frac{4}{3} \frac{g d_{p}}{v_{t}^{2}}\left(\frac{\rho_{p}}{\rho_{f}}-1\right) c_{3} e^{c_{3} T}$

$\rho_{f}=c_{4}-c_{5} T^{2}$

$\rho_{f}=c_{4}$

$\frac{\partial C_{D}}{\partial T}=\frac{4}{3} \frac{g d_{p}}{v_{t}^{2}}\left(\rho_{p}\left(\frac{c_{3} e^{c_{3} T}}{c_{4}-c_{5} T^{2}}+\frac{2 c_{5} T e^{c_{3} T}}{\left(c_{4}-c_{5} T^{2}\right)^{2}}\right)-c_{3} e^{c_{3} T}\right)$

$\frac{\partial C_{D}}{\partial T}=\frac{4}{3} \frac{g d_{p}}{v_{t}^{2}}\left(\left(\frac{\rho_{p}}{\rho_{f}}\right)-1\right) c_{3} e^{c_{3} T}$

OK.

S6.7.5 Fifth term

6.7.5.1 No wall effects correction

$\frac{\partial C_{D}}{\partial v_{t}}=\frac{\partial\left(\frac{\frac{4}{3} g d_{p} \rho_{p}}{v_{t}^{2} \rho_{f}}-\frac{\frac{4}{3} g d_{p}}{v_{t}^{2}}\right)}{\partial v_{t}}$

$\frac{\partial C_{D}}{\partial v_{t}}=\frac{4}{3} g\left(\frac{\rho_{p}}{\rho_{f}}-1\right) \frac{\partial\left(\frac{d_{p}}{v_{t}^{2}}\right)}{\partial v_{t}}$

$\frac{\partial C_{D}}{\partial v_{t}}=\frac{4}{3} g d_{p}\left(\frac{\rho_{p}}{\rho_{f}}-1\right) \frac{\partial\left(v_{t}^{-2}\right)}{\partial v_{t}}$

$\frac{\partial C_{D}}{\partial v_{t}}=-\frac{8}{3} g d_{p}\left(\frac{\rho_{p}}{\rho_{f}}-1\right) v_{t}{ }^{-3}$

Result:

$\frac{\partial C_{D}}{\partial v_{t}}=-\frac{8}{3} g \frac{d_{p}}{v_{t}^{3}}\left(\frac{\rho_{p}}{\rho_{f}}-1\right)$

\subsubsection{With wall effects correction}


$\frac{\partial C_{D}}{\partial v_{t}}=\frac{4}{3} g\left(\frac{\rho_{p}}{\rho_{f}}-1\right) \frac{\partial\left(\frac{d_{p}}{v_{t}^{2}}\right)}{\partial v_{t}}$

$v_{t}=\left(1-c_{2} \frac{d_{p}}{D}\right) v_{t, \infty}$

$\frac{\partial C_{D}}{\partial v_{t}}=\frac{4}{3} g\left(\frac{\rho_{p}}{\rho_{f}}-1\right) \frac{\partial\left(\frac{d_{p}}{\left(\left(1-c_{2} \frac{d_{p}}{D}\right) v_{t, \infty}\right)^{2}}\right)}{\partial v_{t}}$

$\frac{\partial C_{D}}{\partial v_{t}}=\frac{4}{3} g\left(\frac{\rho_{p}}{\rho_{f}}-1\right) \frac{\partial\left(\frac{d_{p}}{\left(v_{t}-c_{2} \frac{d_{p} v_{t}}{D}\right)^{2}}\right)}{\partial v_{t}}$

$\frac{\partial C_{D}}{\partial v_{t}}=\frac{4}{3} g d_{p}\left(\frac{\rho_{p}}{\rho_{f}}-1\right) \frac{\partial\left(\left(v_{t}\left(1-c_{2} \frac{d_{p}}{D}\right)\right)^{-2}\right)}{\partial v_{t}}$

$\frac{\partial C_{D}}{\partial v_{t}}=-\frac{8}{3} g d_{p}\left(\frac{\rho_{p}}{\rho_{f}}-1\right)\left(v_{t}\left(1-c_{2} \frac{d_{p}}{D}\right)\right)^{-3} \frac{\partial\left(v_{t}\left(1-c_{2} \frac{d_{p}}{D}\right)\right)}{\partial v_{t}}$

$\frac{\partial C_{D}}{\partial v_{t}}=-\frac{8}{3} g d_{p}\left(\frac{\rho_{p}}{\rho_{f}}-1\right)\left(v_{t}\left(1-c_{2} \frac{d_{p}}{D}\right)\right)^{-3}\left(1-c_{2} \frac{d_{p}}{D}\right)$

Result:

$$
\frac{\partial C_{D}}{\partial v_{t}}=-\frac{8}{3} \frac{g d_{p}}{v_{t}^{3}} \frac{\left(\frac{\rho_{p}}{\rho_{f}}-1\right)}{\left(1-c_{2} \frac{d_{p}}{D}\right)^{2}}
$$

\subsubsection{Check: no wall effects}

$$
\frac{\partial C_{D}}{\partial v_{t}}=-\frac{8}{3} \frac{g d_{p}}{v_{t}^{3}} \frac{\left(\frac{\rho_{p}}{\rho_{f}}-1\right)}{\left(1-c_{2} \frac{d_{p}}{D}\right)^{2}}
$$


$\frac{\partial C_{D}}{\partial v_{t}}=-\frac{8}{3} \frac{g d_{p}}{v_{t}{ }^{3}}\left(\frac{\rho_{p}}{\rho_{f}}-1\right)$

OK.

\section{S6.8 Particle density uncertainty}

Accordingly, the error (uncertainty $\delta$ ) considering the particle density $\rho_{p}$ can be determined. The estimate of uncertainty is given by the following expressions:

$$
\delta \rho_{p}=\sqrt{\left(\frac{\partial \rho_{p}}{\partial m_{p}} \delta m_{p}\right)^{2}+\left(\frac{\partial \rho_{p}}{\partial d_{p}} \delta d_{p}\right)^{2}}
$$

Given:

$$
\rho_{p}=\frac{6 m_{p}}{\pi d_{p}{ }^{3}}
$$

\section{S6.8.1 First term}

$$
\begin{aligned}
& \frac{\partial \rho_{p}}{\partial m_{p}}=\frac{\partial\left(\frac{6 m_{p}}{\pi d_{p}^{3}}\right)}{\partial m_{p}} \\
& \frac{\partial \rho_{p}}{\partial m_{p}}=\frac{6}{\pi d_{p}{ }^{3}}
\end{aligned}
$$

\section{S6.8.2 Second term}

$$
\frac{\partial \rho_{p}}{\partial d_{p}}=\frac{\partial\left(\frac{6 m_{p}}{\pi d_{p}^{3}}\right)}{\partial d_{p}}
$$

$\frac{\partial \rho_{p}}{\partial d_{p}}=\frac{6 m_{p}}{\pi} \frac{\partial\left(\frac{1}{d_{p}^{3}}\right)}{\partial d_{p}}$

$\frac{\partial \rho_{p}}{\partial d_{p}}=-\frac{6 m_{p}}{\pi d_{p}{ }^{4}}$ 
Result:

$$
\delta \rho_{p}=\sqrt{\left(\frac{6}{\pi d_{p}{ }^{3}} \delta m_{p}\right)^{2}+\left(-\frac{6 m_{p}}{\pi d_{p}{ }^{4}} \delta d_{p}\right)^{2}}
$$

\section{S6.9 Terminal settling velocity uncertainty}

What is the error (uncertainty $\delta$ ) in the terminal settling velocity $v_{t}$ :

$$
\delta v_{t}=\sqrt{\left(\frac{\partial v_{t}}{\partial L} \delta L\right)^{2}+\left(\frac{\partial v_{t}}{\partial t} \delta t\right)^{2}}
$$

with

$$
L=v_{t} t
$$

\section{S6.9.1 First term}

$$
\begin{aligned}
& \frac{\partial v_{t}}{\partial L}=\frac{\partial\left(\frac{L}{t}\right)}{\partial L} \\
& \frac{\partial v_{t}}{\partial L}=\frac{1}{t} \frac{\partial(L)}{\partial L} \\
& \frac{\partial v_{t}}{\partial L}=\frac{1}{t}
\end{aligned}
$$

\section{S6.9.2 Second term}

$$
\begin{aligned}
& \frac{\partial v_{t}}{\partial L}=\frac{\partial\left(\frac{L}{t}\right)}{\partial t} \\
& \frac{\partial v_{t}}{\partial L}=L \frac{\partial\left(\frac{1}{t}\right)}{\partial t} \\
& \frac{\partial v_{t}}{\partial L}=-L \frac{1}{t^{2}}
\end{aligned}
$$


Result:

$$
\delta v_{t}=\sqrt{\left(\frac{1}{t} \delta L\right)^{2}+\left(-\frac{L}{t^{2}} \delta t\right)^{2}}
$$

\section{S6.9.3 Relative velocity:}

Given:

$$
\frac{1}{v_{t}}=\frac{t}{L}
$$

Rewriting:

$$
\begin{aligned}
& \frac{\delta v_{t}}{v_{t}}=\frac{1}{v_{t}} \sqrt{\left(\frac{1}{t} \delta L\right)^{2}+\left(-\frac{L}{t^{2}} \delta t\right)^{2}} \\
& \frac{\delta v_{t}}{v_{t}}=\sqrt{\left(\frac{1}{v_{t}} \frac{1}{t} \delta L\right)^{2}+\left(-\frac{1}{v_{t}} \frac{L}{t^{2}} \delta t\right)^{2}}
\end{aligned}
$$

with

$$
L=v_{t} t
$$

$$
\frac{\delta v_{t}}{v_{t}}=\sqrt{\left(\frac{1}{L} \delta L\right)^{2}+\left(-\frac{L}{L^{2}} \delta t\right)^{2}}
$$

Result:

$$
\frac{\delta v_{t}}{v_{t}}=\sqrt{\left(\frac{\delta L}{L}\right)^{2}+\left(-\frac{\delta t}{L}\right)^{2}}
$$




\section{S6.9.4 Human response time inaccuracy correction}

To compensate the larger inaccuracy of the measured time using a stopwatch, in particular when the particle settling velocity is high as well when the fall height is relatively short, a 'human response time' empirical correction equation is introduced:

$$
\delta t=c_{0}+c_{1} e^{-t}
$$

where $c_{0}$ is the fastest human reaction time, which is approximately $15 / 100 \mathrm{~s} . c_{1}$, is estimated on and set to a relatively low value of 0.5 to reduce the degree of corrections. For $N=3,629$ experiments: $\delta t=16 / 100 \pm 2 / 100$.

\section{S6.10 Uncertainty analysis parameters and coefficients}

\section{Table S20 Parameters of equations}

\begin{tabular}{ll}
\hline Parameter & Value \\
\hline $\mathrm{c}_{0}$ & $1.651 \cdot 10^{-6}$ \\
$\mathrm{c}_{1}$ & -0.02358 \\
$\mathrm{c}_{2}$ & 2.1 \\
$\mathrm{c}_{3}$ & $2.499 \cdot 10^{-5}$ \\
$\mathrm{C}_{4}$ & 1000 \\
$\mathrm{C}_{5}$ & 0.004715 \\
$\mathrm{c}_{6}$ & $2.414 \cdot 10^{-8}$ \\
$\mathrm{C}_{7}$ & 247.8 \\
$\mathrm{c}_{8}$ & 133.15 \\
\hline
\end{tabular}

Table S21 Linear heat expansion coefficients (Rumble, 2019); (Perry and Green, 2007)

\section{$\alpha \cdot \mathbf{1 0}^{-6}[\mathrm{~m} / \mathrm{mK}] \quad$ Value}

\begin{tabular}{ll}
\hline Calcite & 25 \\
Garnet & 7 \\
GAC & $4-8$ \\
Crystal sand & 10 \\
Rapid filter sand & $8-14$ \\
Glass pearls & 6 \\
Metal balls & $10.8-12.5$ \\
IEX balls & $50-90^{1)}$ \\
Zirconium & 5.7 \\
Synthetic material & $50-90$ \\
Calcite & 25 \\
Garnet & 7 \\
GAC & $4-8$ \\
Crystal sand & 10 \\
\hline 1) www.engineeringtoolbox.com
\end{tabular}




\section{S7.1 Natural and processed highly non-spherical polydisperse particles}

The first examined grains caused the largest degree of spread in the standard drag curve, which is visible in Figure S42 but very manifest in Figure S43 where the logarithmic scales (log-log) are replaced by Cartesian coordinate scales (lin-lin).

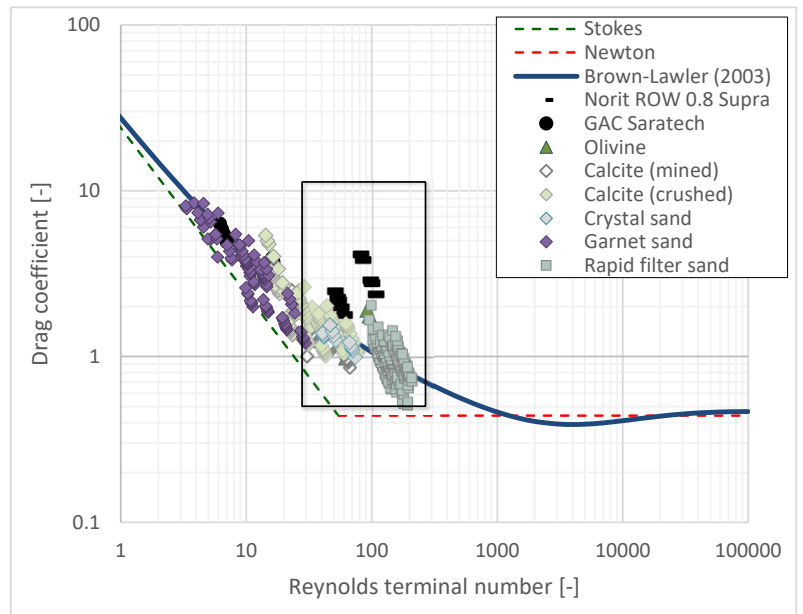

Figure S42 SDC (log-log) natural irregularly shaped particles often applied in water treatment processes, olivine $G A C$, calcite and sand types

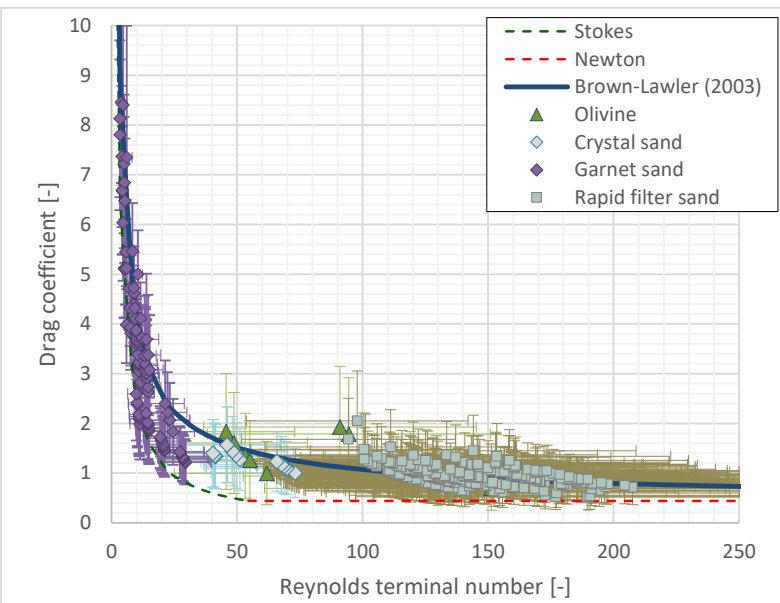

Figure S44 SDC (lin-lin) olivine, crystal sand, garnet sand and rapid filter sand with (uncertainty) error bars $\left(C_{D}\right.$ and $\left.R_{t}\right)$

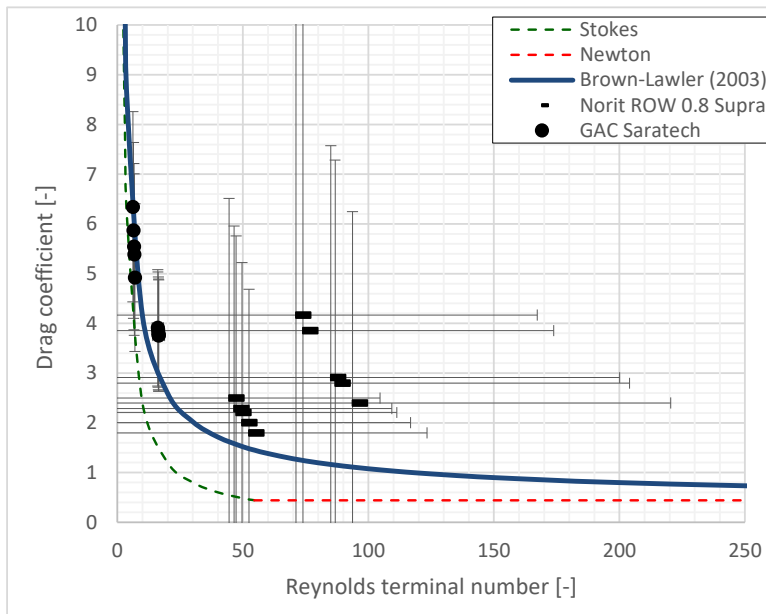

Figure S43 SDC (lin-lin) selection of GAC grains with (uncertainty) error bars $\left(C_{D}\right.$ and $\left.R e_{t}\right)$

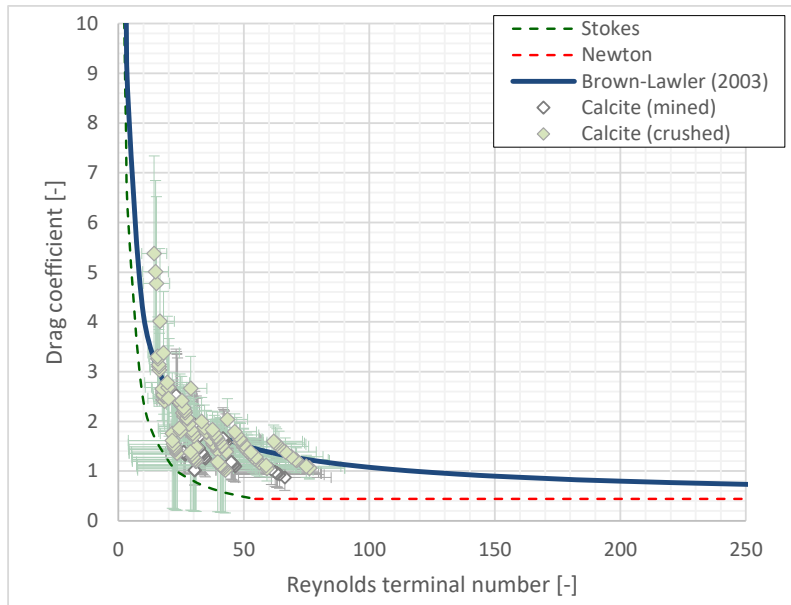

Figure S45 SDC (lin-lin) crushed calcite with (uncertainty) error bars $\left(C_{D}\right.$ and $\left.R_{t}\right)$

\section{S7.2 Medium non-spherical polydisperse particles applied in water softening}

Figure S46, however, shows a considerably larger disagreement with the Brown-Lawler prediction. The error bars in Figure S47, based on the uncertainty analysis, decrease as the calcite pellets become larger. This can be explained as smaller calcite pellets are more irregularly shaped. Due to particle growth in the softening process, particles become more spherical (increased sphericity $\Phi$ ) and hence demonstrate a decreased degree of spread. 


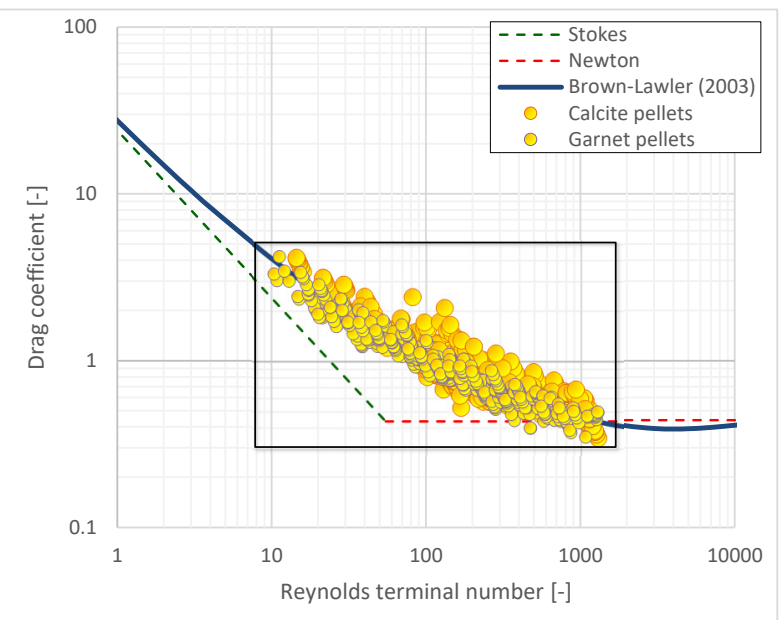

Figure S46 SDC (log-log) 0.25-4.0 mm garnet pellets and 0.353.36 calcite pellets

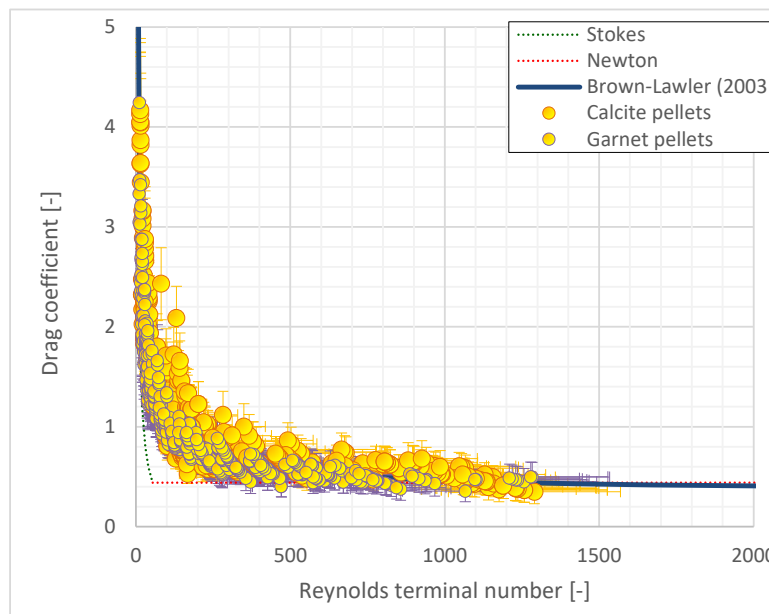

Figure $\mathbf{S 4 7}$ SDC (lin-lin) selection with (uncertainty) error bars $\left(C_{D}\right.$ and $\left.R e_{t}\right)$

\section{S7.3 Spherical polydisperse and monodisperse glass beads}

In the literature, glass beads are popular and frequently applied for model calibration and validation purposes. In this work 288 individual spherical glass pearls were settled. Figure S48, with $\log$-log scales, shows reasonable agreement with the Brown-Lawler curve. In Figure S49, the spread remains visible due to the polydispersity (UC $>1$ ), albeit less significant.

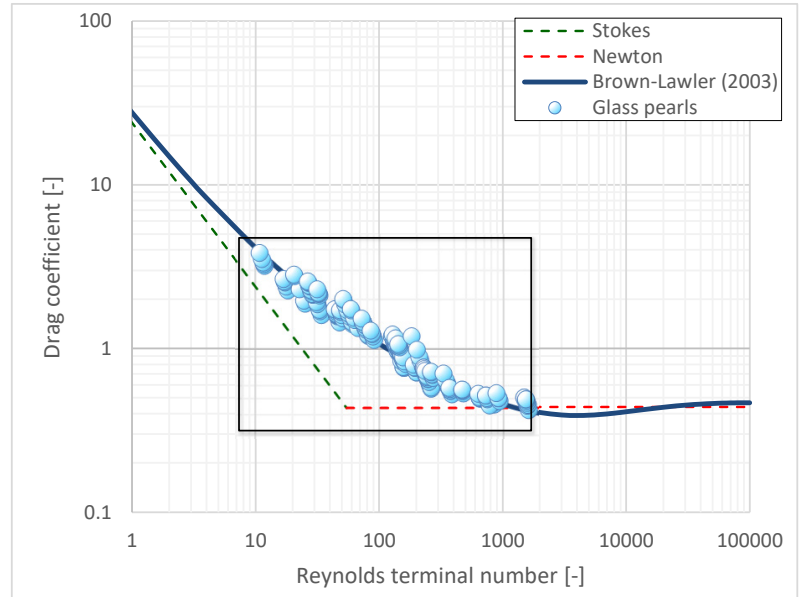

Figure $\mathbf{S 4 8} S D C$ (log-log) 0.25-3.15 $\mathrm{mm}$ spherical polydisperse glass beads

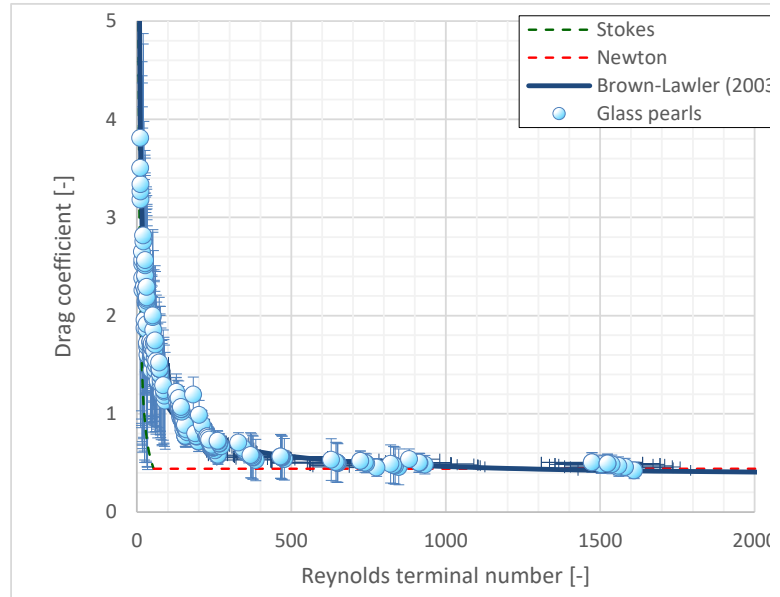

Figure $\mathbf{S 4 9}$ SDC (lin-lin) selection with (uncertainty) error bars $\left(C_{D}\right.$ and $\left.R_{t}\right)$ 


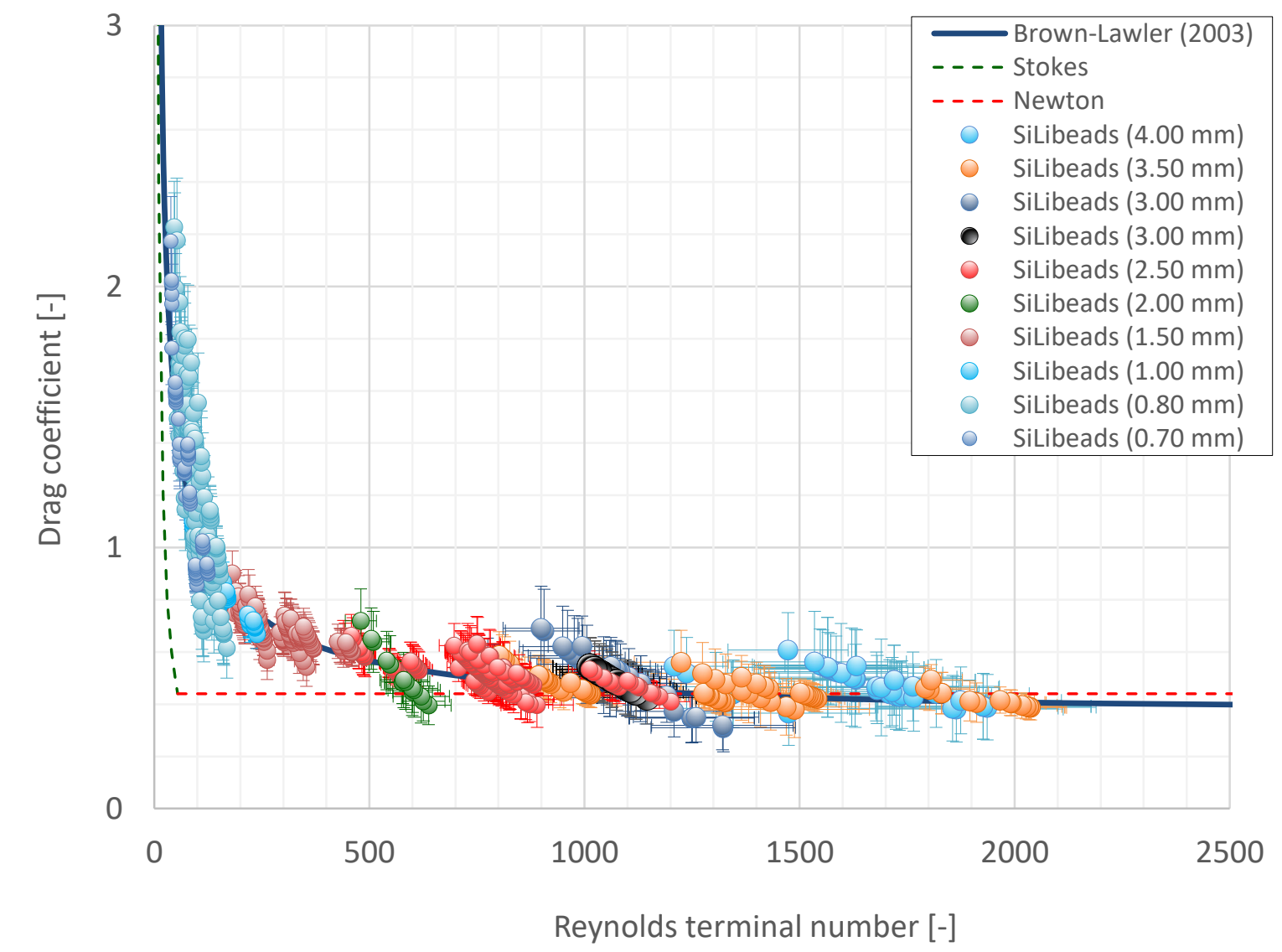

Figure S50 SDC (lin-lin) 0.7, 0.8, 1.0, 1.5, 2.0, 2.5, 3.0, 3.5 and $4.0 \mathrm{~mm}$ SiLibeads spherical monodisperse glass beads with (uncertainty) error bars $\left(C_{D}\right.$ and $\left.R_{t}\right)$

\section{S7.4 Repetitive experiment with highly spherical monodisperse single glass beads}

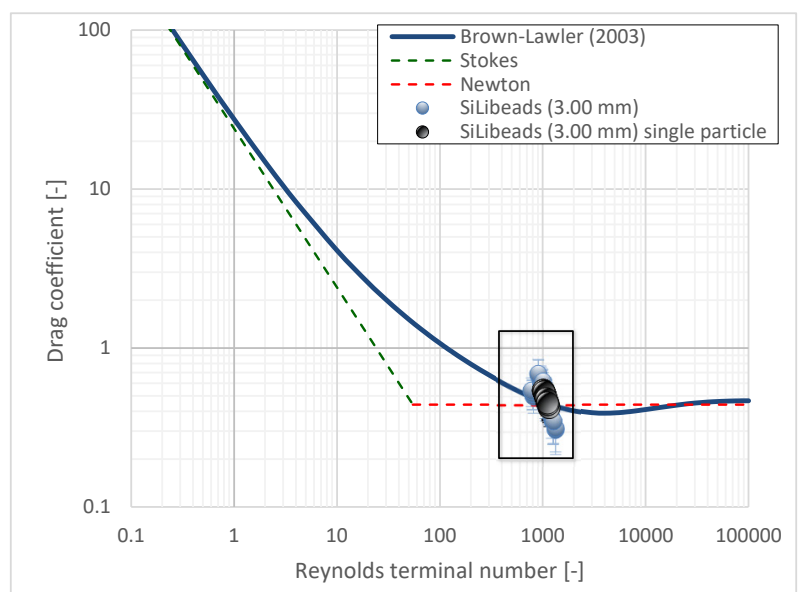

Figure S51 SDC (log-log) $3.0 \mathrm{~mm}$ SiLibeads spherical monodisperse glass beads standard settling experiment (blue) and repeated with one and the same single grain plus trajectory recording (black)

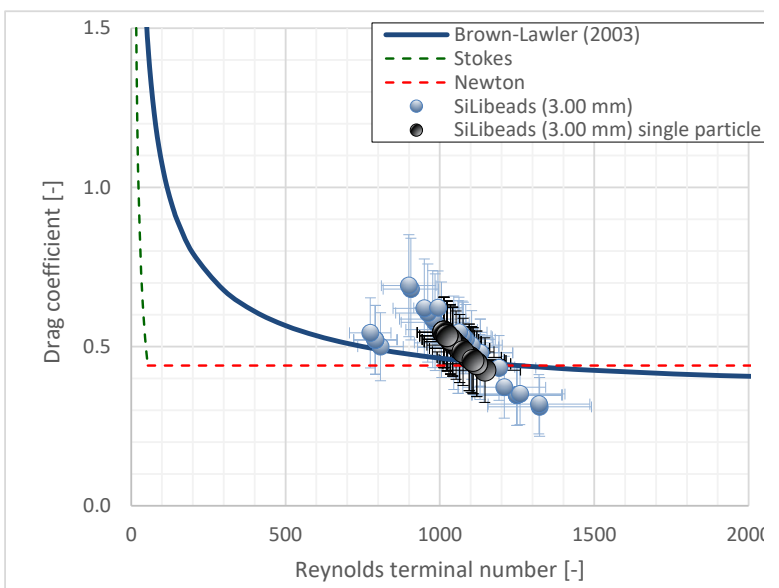

Figure S52 SDC (lin-lin) selection $3.0 \mathrm{~mm}$ SiLibeads spherical monodisperse glass beads with (uncertainty) error bars $\left(C_{D}\right.$ and $\left.R_{t}\right)$ 


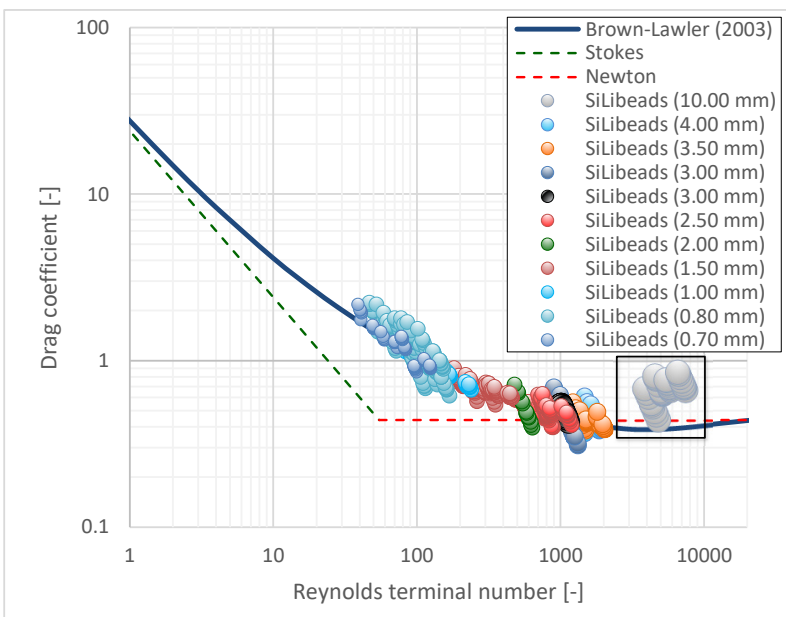

Figure S53 SDC (log-log) 0.7-10.0 mm SiLibeads spherical monodisperse glass beads

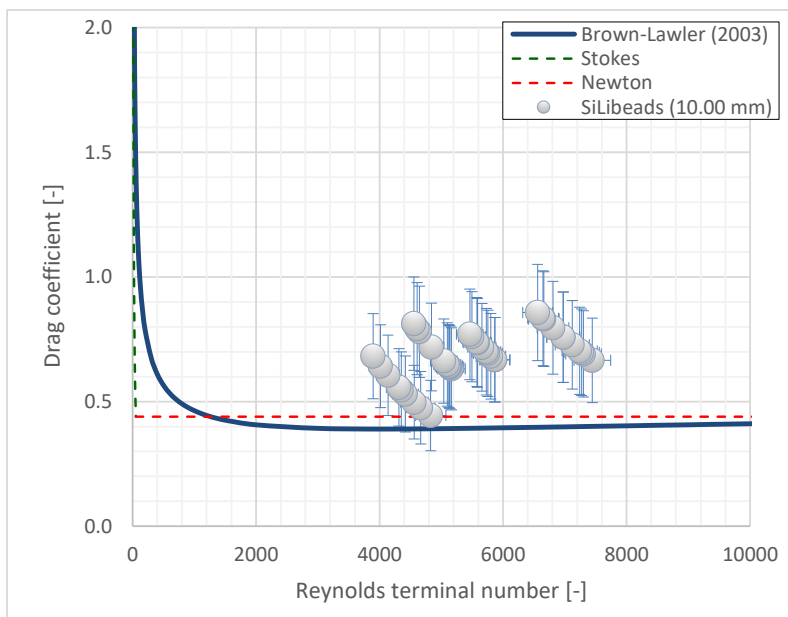

Figure S54 SDC (lin-lin) selection with (uncertainty) error bars $\left(C_{D}\right.$ and $\left.R_{t}\right)$ with wall effects

\section{S7.6 Highly spherical monodisperse glass beads in different columns}

To explore the influence of the column diameter, the same experiments were executed in two columns with different sizes $(D$ $=57 \mathrm{~mm})$ and $(D=125 \mathrm{~mm})$, indicated with $\circ$ and $\square$ for three different glass bead sizes $(1.5,2.5$ and $3.5 \mathrm{~mm})$. Based on the results in Figure S55 and Figure S56, no distinction can be made.

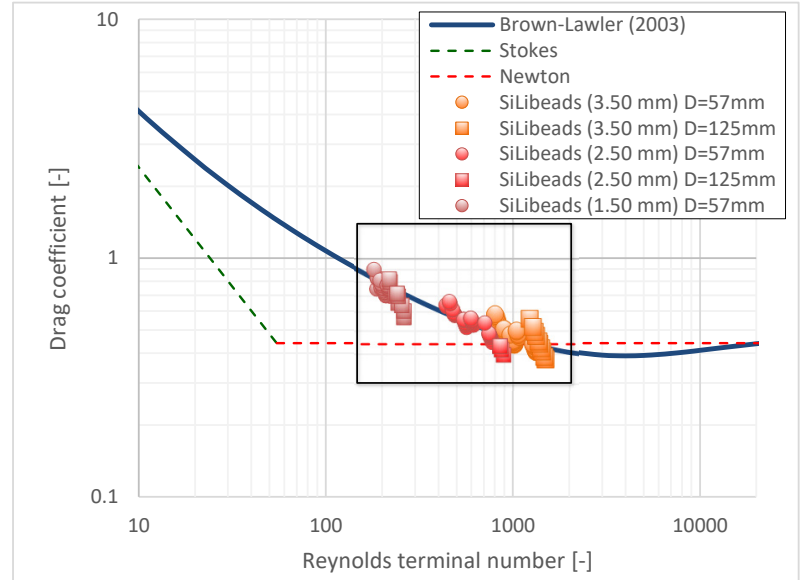

Figure S55 SDC (log-log) 1.5, 2.5 and $3.5 \mathrm{~mm}$ SiLibeads spherical monodisperse glass beads standard settling experiment in two cylindrical columns 57 and $125 \mathrm{~mm}$

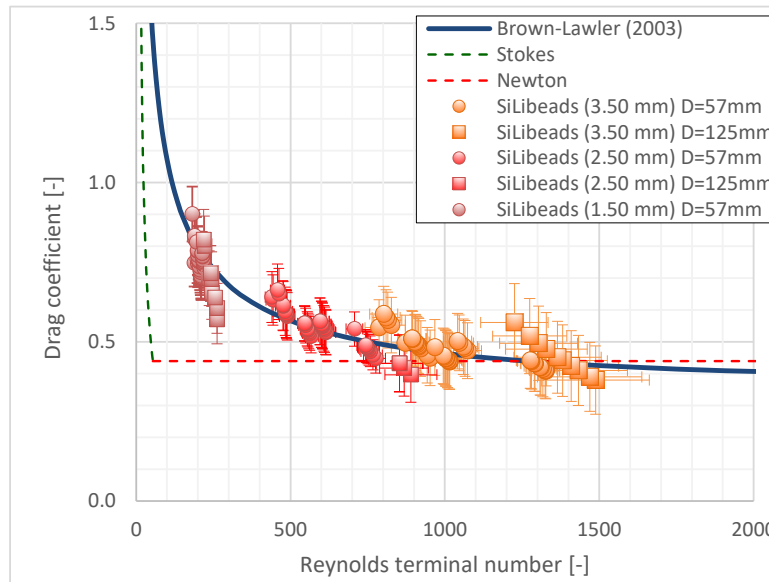

Figure S56 SDC (lin-lin) selection SiLibeads spherical monodisperse glass beads with (uncertainty) error bars $\left(C_{D}\right.$ and $\left.R e_{t}\right)$ 


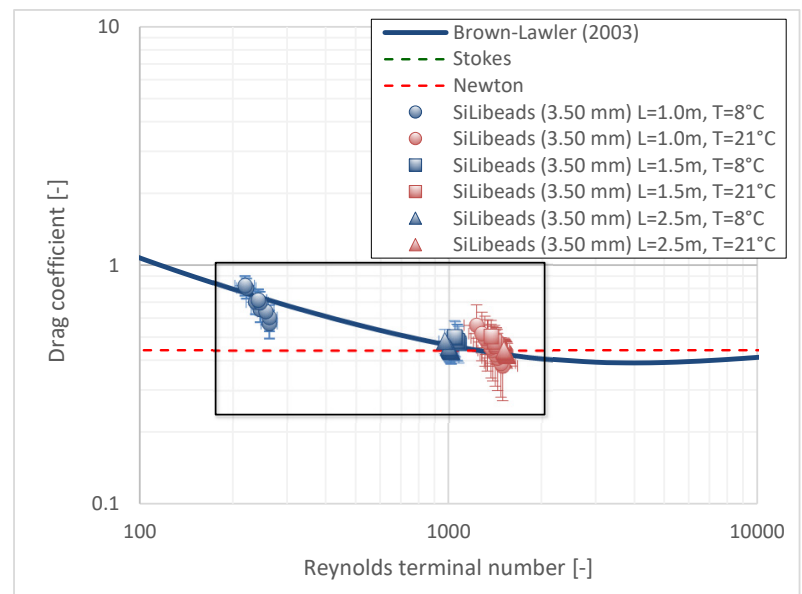

Figure S57 SDC (log-log) $3.5 \mathrm{~mm}$ SiLibeads spherical monodisperse glass beads with different fall lengths

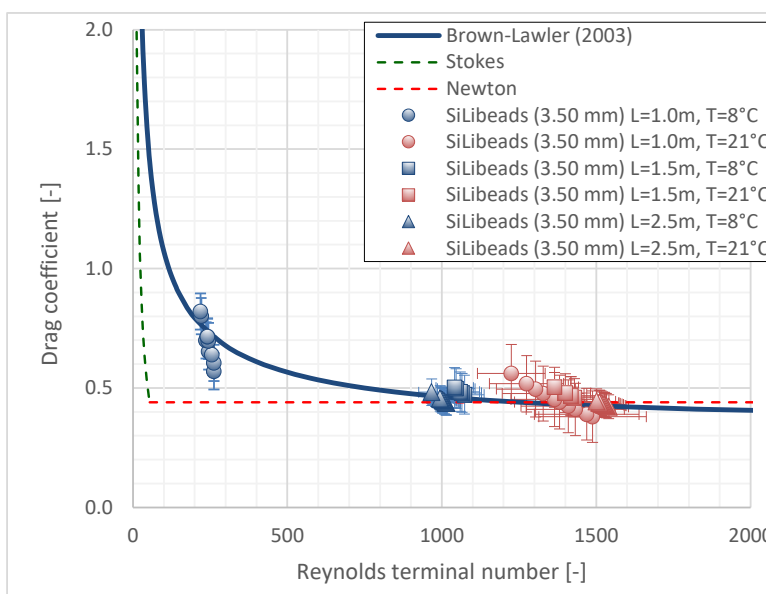

Figure S58 SDC (lin-lin) selection SiLibeads spherical monodisperse glass beads with (uncertainty) error bars $\left(C_{D}\right.$ and $\left.R e_{t}\right)$

\section{S7.8 Highly spherical polydisperse synthetic particles}

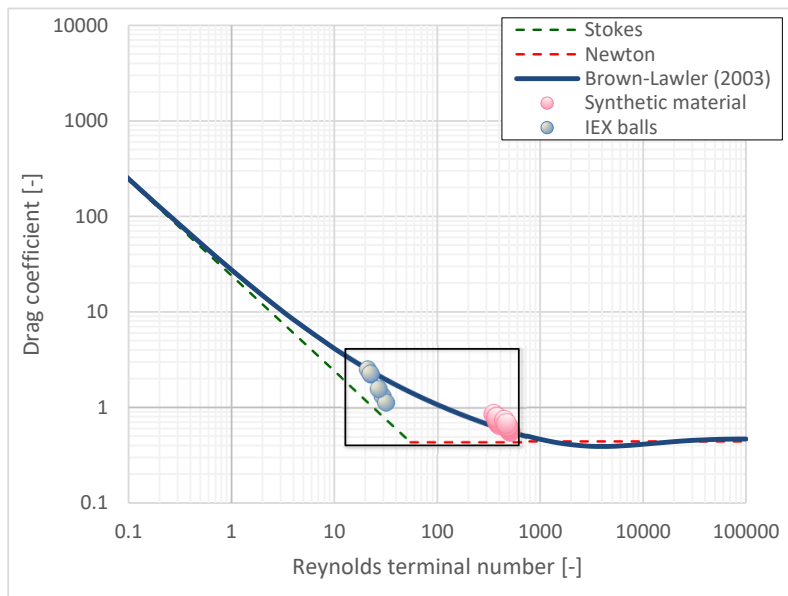

Figure S59 SDC (log-log) 0.9-1.18 $\mathrm{mm}$ IEX resin balls and $3.0 \mathrm{~mm}$ synthetic nylon balls

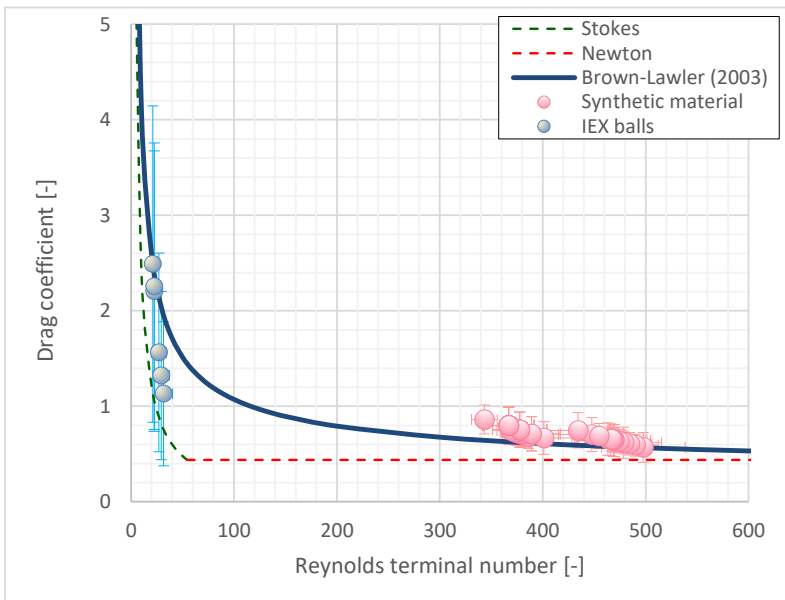

Figure S60 SDC (lin-lin) selection with (uncertainty) error bars $\left(C_{D}\right.$ and $\left.R e_{t}\right)$ with subdivision in settling behaviour 


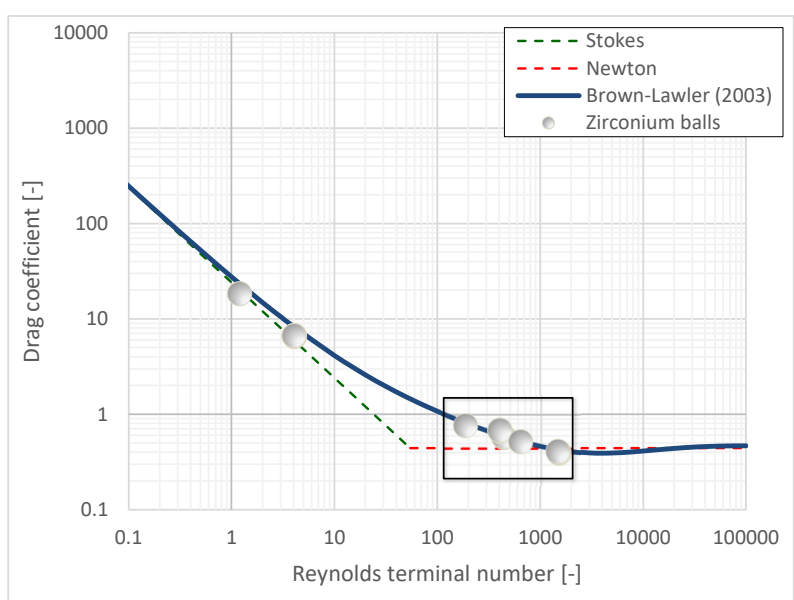

Figure S61 SDC (log-log) 0.1, 1.0 and $2.0 \mathrm{~mm}$ zirconium balls

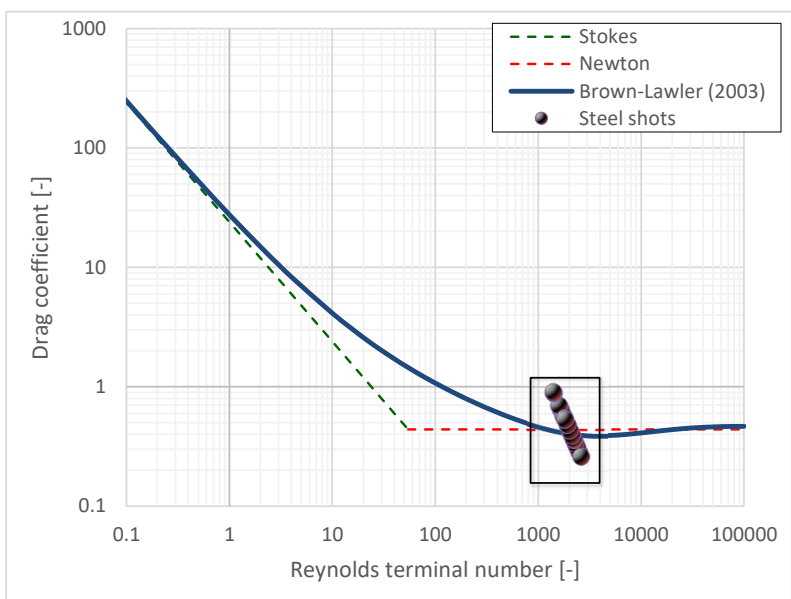

Figure S63 SDC (log-log) $3.0 \mathrm{~mm}$ steel shots

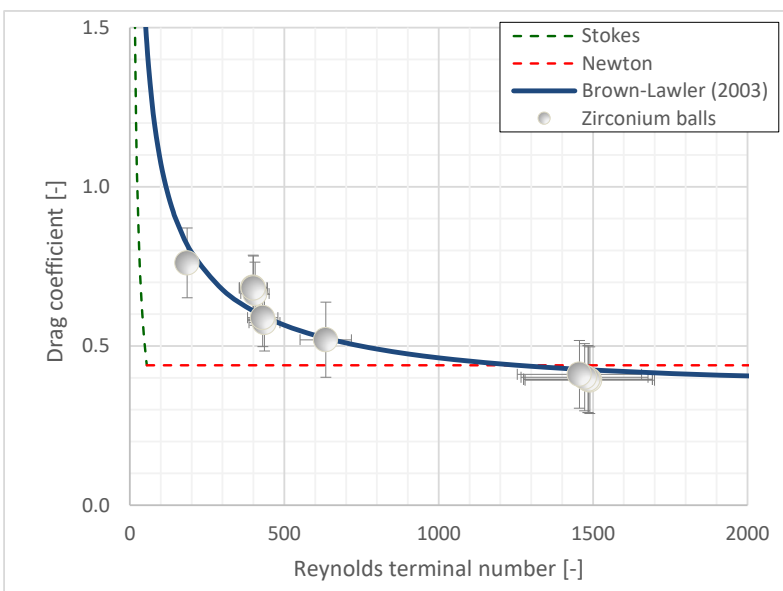

Figure S62 SDC (lin-lin) selection with (uncertainty) error bars $\left(C_{D}\right.$ and $\left.R e_{t}\right)$ with subdivision in settling behaviour

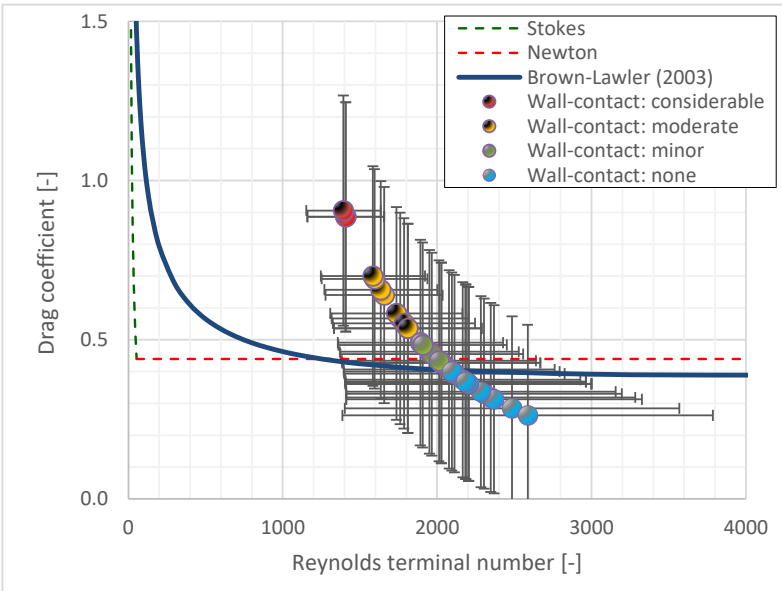

Figure S64 SDC (lin-lin) selection with (uncertainty) error bars $\left(C_{D}\right.$ and $\left.R_{t}\right)$ with subdivision in settling behaviour 


\section{S8 Recorded path instabilities}

\section{S8.1 Path trajectories}

Recorded path trajectories of fractionated calcite pellets from $1.0 \mathrm{~mm}-2.8 \mathrm{~mm}$ using an advanced experimental set-up (Figure S5 and Figure S6), discussed in detail by (Raaghav, 2019). Videos can be found at (Kramer et al., 2020c).

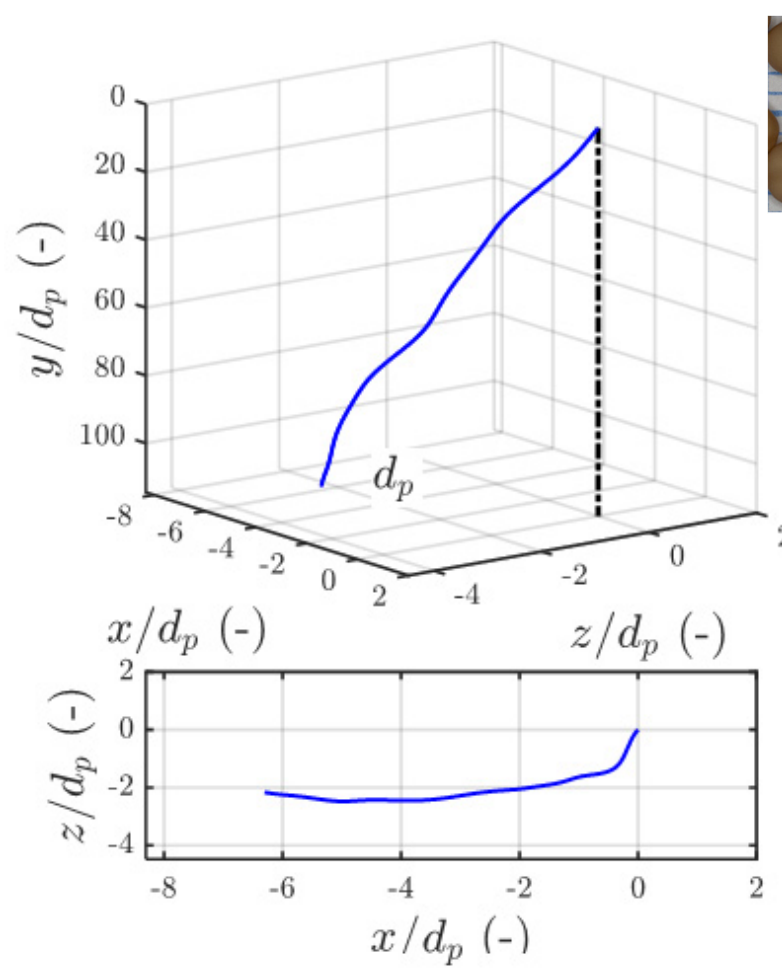

Figure S65 Calcite pellets: $d_{p}>2.8 \mathrm{~mm}, \mathrm{~T}=20^{\circ} \mathrm{C}$, $C_{D}=0.49, \bar{\rho}=2.7, R e_{t}=978, G a=593$, $v_{t}=0.35 \mathrm{~m} / \mathrm{s}, v_{t, B L}=0.39 \mathrm{~m} / \mathrm{s}$, angle $=3.4^{\circ}$, $-\% v_{t}=9 \%$, path: $\mathrm{CH}$
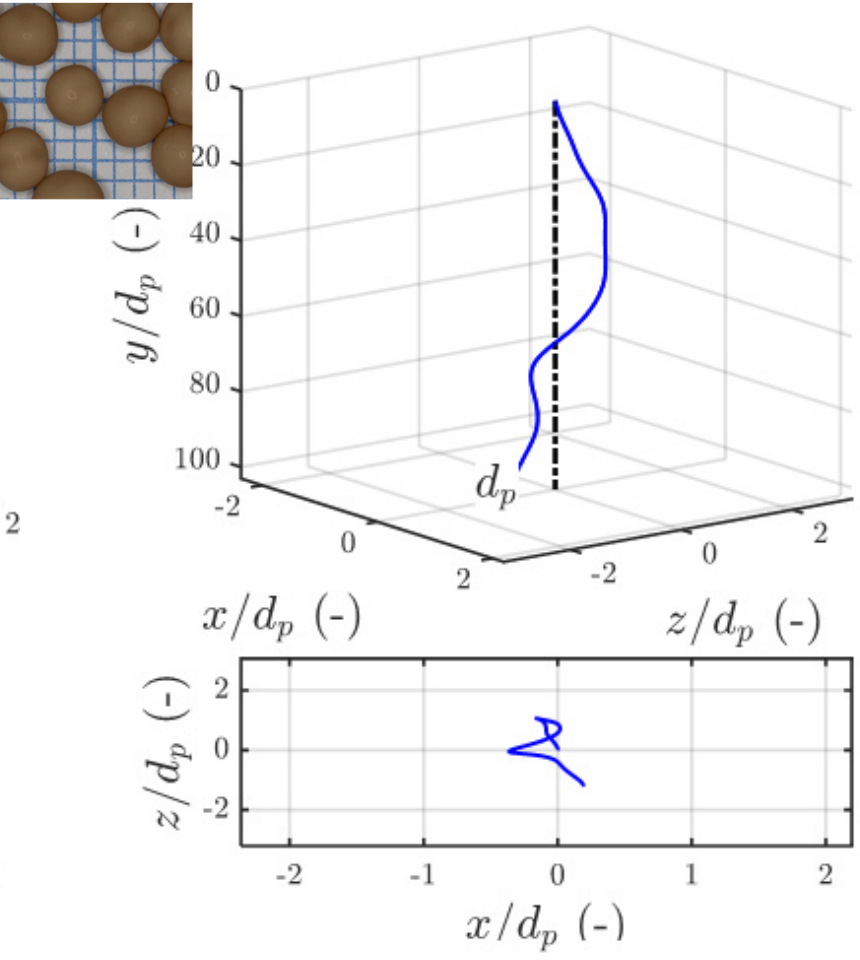

Figure $\mathbf{S 6 6}$ Calcite pellets: $d_{p}>2.8 \mathrm{~mm}, \mathrm{~T}=20^{\circ} \mathrm{C}$, $C_{D}=0.52, \bar{\rho}=2.7, R_{t}=950, G a=593$, $v_{t}=0.34 \mathrm{~m} / \mathrm{s}, v_{t, B L}=0.39 \mathrm{~m} / \mathrm{s}$, angle $=1.0^{\circ}$, $-\% v_{t}=11 \%$, path: $\mathrm{CH}$ 

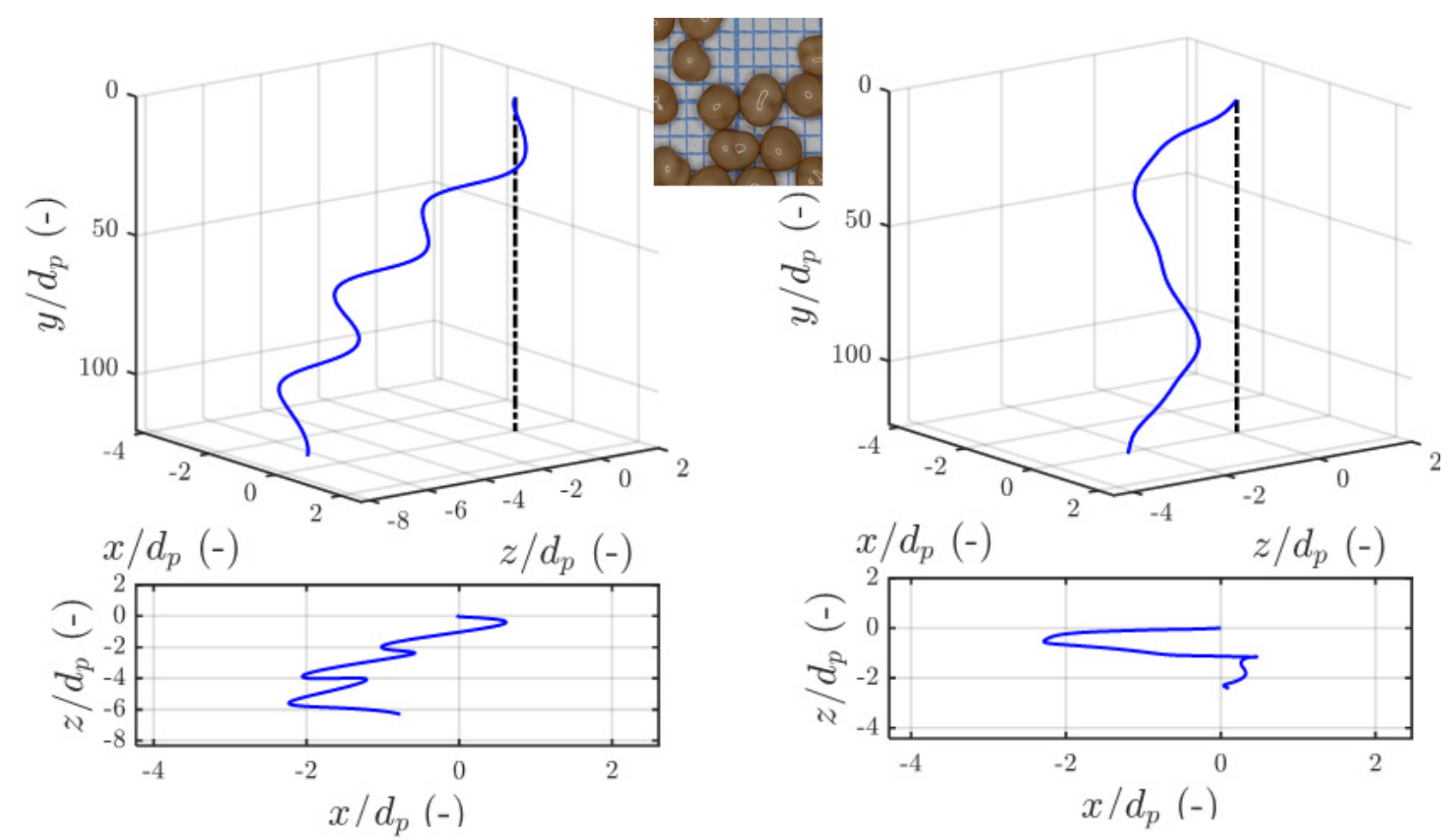

Figure $\mathbf{S 6 7}$ Calcite pellets: $2.36<d_{p}<2.8 \mathrm{~mm}, \mathrm{~T}=20{ }^{\circ} \mathrm{C}$, $C_{D}=0.55, \bar{\rho}=2.7, R e_{t}=809, G a=522$,

$v_{t}=0.32 \mathrm{~m} / \mathrm{s}, v_{t, B L}=0.34 \mathrm{~m} / \mathrm{s}$, angle $=2.8^{\circ}$,

$-\% v_{t}=7 \%$, path: $\mathrm{CH}$

Figure $\mathbf{S 6 8}$ Calcite pellets: $2.36<d_{p}<2.8, T=20^{\circ} \mathrm{C}$, $C_{D}=0.57, \bar{\rho}=2.7, R_{t}=800, G a=522$, $v_{t}=0.32 \mathrm{~m} / \mathrm{s}, v_{t, B L}=0.34 \mathrm{~m} / \mathrm{s}$, angle $=2.1^{\circ}$, $-\% v_{t}=8 \%$, path: $\mathrm{CH}$ 

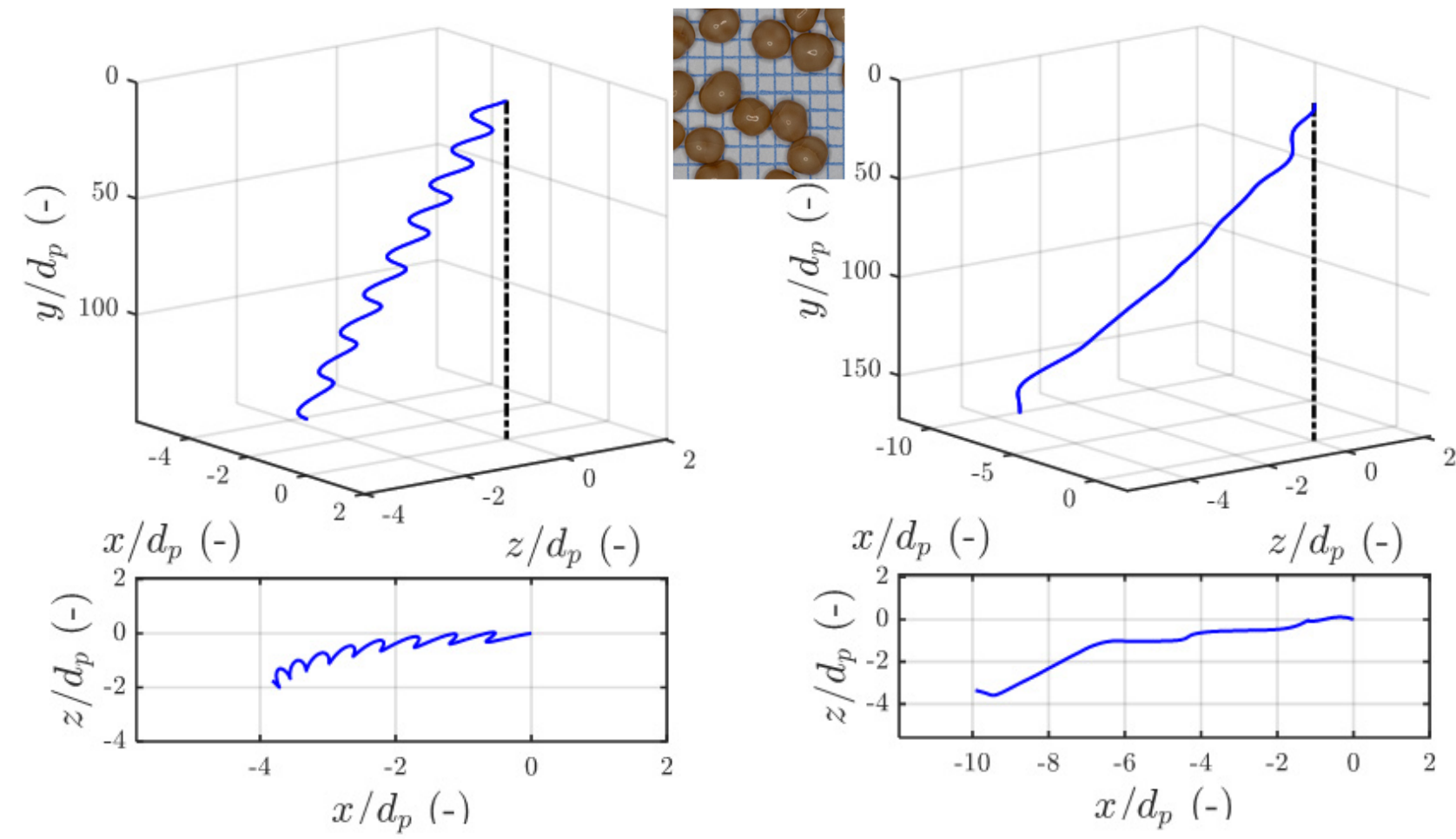

Figure $\mathbf{S 6 9}$ Calcite pellets: $2.0<d_{p}<2.36 \mathrm{~mm}, T=20{ }^{\circ} \mathrm{C}$, $C_{D}=0.75, \bar{\rho}=2.7, R e_{t}=540, G a=406$, $v_{t}=0.25 \mathrm{~m} / \mathrm{s}, v_{t, B L}=0.30 \mathrm{~m} / \mathrm{s}$, angle $=2.3^{\circ}$, $-\% v_{t}=17 \%$, path: $\mathrm{CH}$

Figure S70 Calcite pellets: $2.0<d_{p}<2.36, T=20{ }^{\circ} \mathrm{C}$, $C_{D}=0.76, \bar{\rho}=2.7, R_{t}=535, G a=406$, $v_{t}=0.25 \mathrm{~m} / \mathrm{s}, v_{t, B L}=0.30 \mathrm{~m} / \mathrm{s}$, angle $=3.4^{\circ}$, $-\% v_{t}=17 \%$, path: $\mathrm{CH}$ 

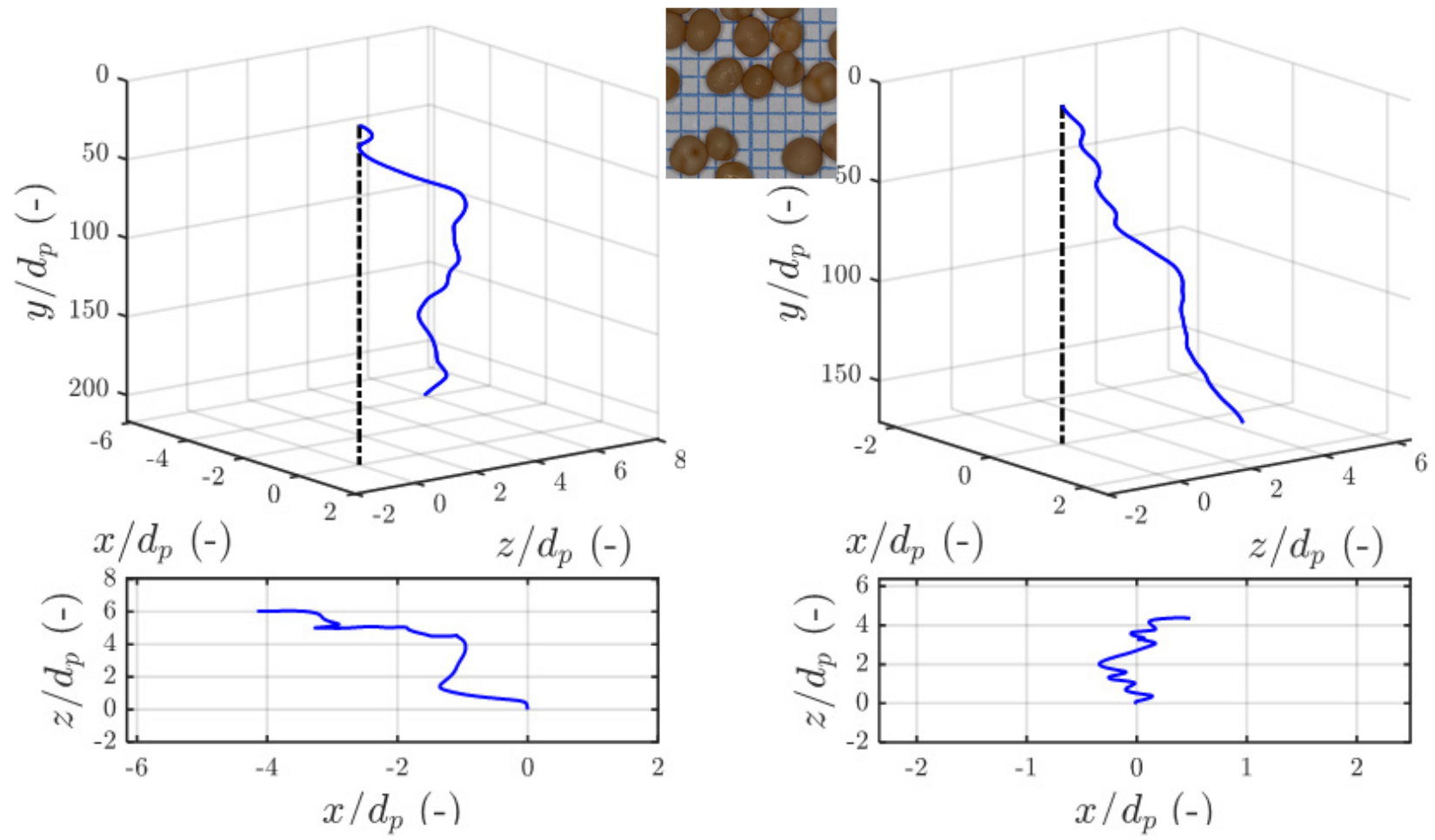

Figure $\boldsymbol{S 7 1}$ Calcite pellets: $1.7<d_{p}<2 \mathrm{~mm}, \mathrm{~T}=20{ }^{\circ} \mathrm{C}$, $C_{D}=0.62, \bar{\rho}=2.7, R e_{t}=465, G a=317$, $v_{t}=0.26 \mathrm{~m} / \mathrm{s}, v_{t, B L}=0.27 \mathrm{~m} / \mathrm{s}$, angle $=2.8^{\circ}$, $-\% v_{t}=4 \%$, path: $\mathrm{CH}$

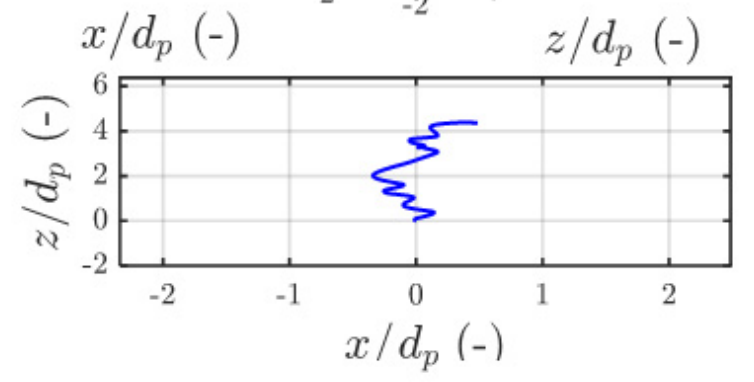

Figure $\mathbf{S 7 2}$ Calcite pellets: $1.7<d_{p}<2 \mathrm{~mm}, \mathrm{~T}=20{ }^{\circ} \mathrm{C}$, $C_{D}=0.93, \bar{\rho}=2.7, R_{t}=380, G a=317$, $v_{t}=0.21 \mathrm{~m} / \mathrm{s}, v_{t, B L}=0.27 \mathrm{~m} / \mathrm{s}$, angle $=1.6^{\circ}$, $-\% v_{t}=22 \%$, path: $\mathrm{CH}$ 

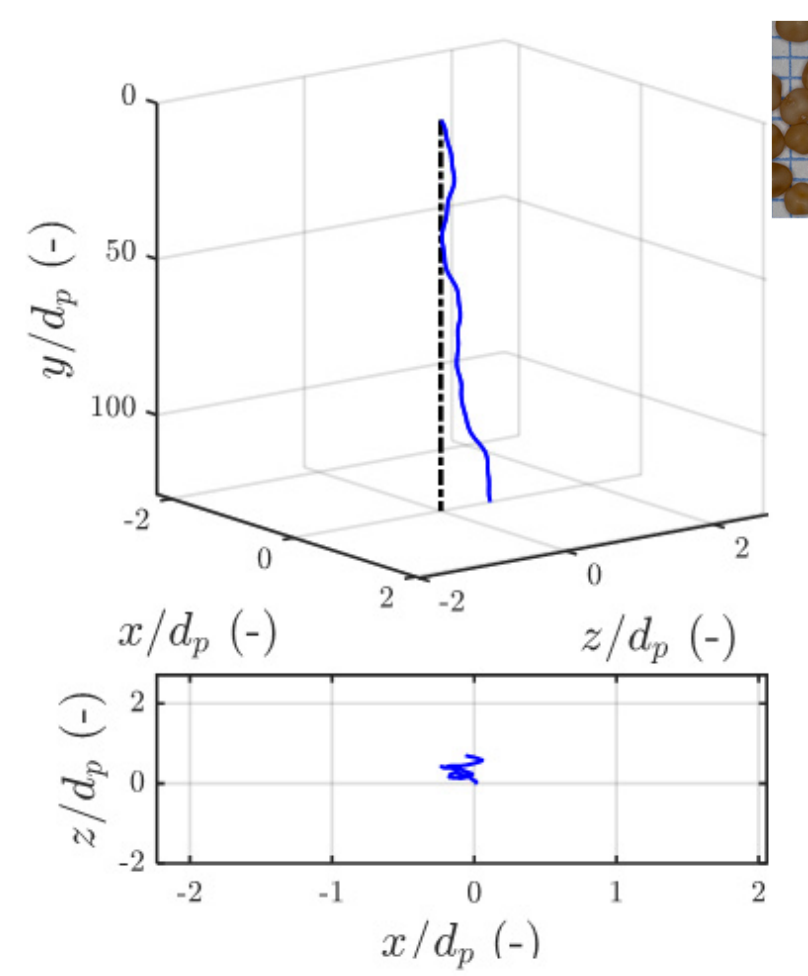

Figure $\mathbf{S 7 3}$ Calcite pellets: $1.4<d_{p}<1.7 \mathrm{~mm}, \mathrm{~T}=20^{\circ} \mathrm{C}$, $C_{D}=0.80, \bar{\rho}=2.7, R e_{t}=314, G a=243$, $v_{t}=0.21 \mathrm{~m} / \mathrm{s}, v_{t, B L}=0.23 \mathrm{~m} / \mathrm{s}$, angle $=0.4^{\circ}$, $-\% v_{t}=10 \%$, path: $\mathrm{CH}$
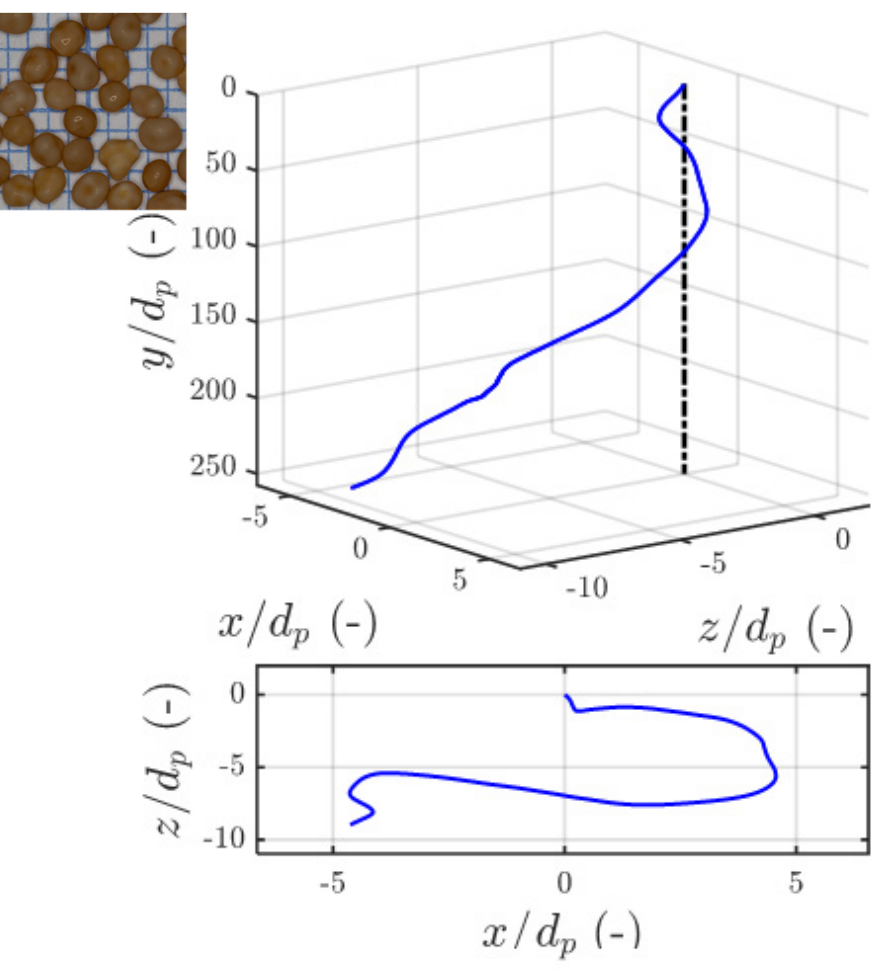

Figure $\mathbf{S 7 4}$ Calcite pellets: $1.4<d_{p}<1.7 \mathrm{~mm}, \mathrm{~T}=20^{\circ} \mathrm{C}$, $C_{D}=0.71, \bar{\rho}=2.7, R_{t}=332, G a=243, v_{t}=0.22$ $\mathrm{m} / \mathrm{s}, v_{t, B L}=0.23 \mathrm{~m} / \mathrm{s}$, angle $=2.9^{\circ},-\% v_{t}=5 \%$, path: $\mathrm{CH}$ 

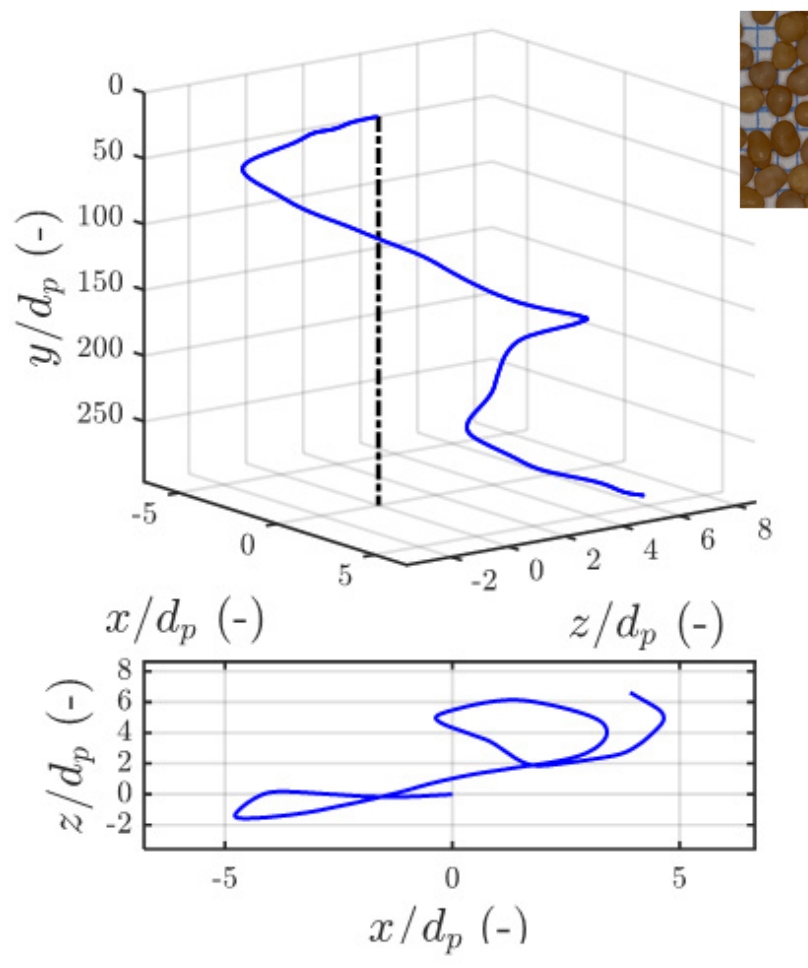

Figure $\mathbf{S 7 5}$ Calcite pellets: $1.25<d_{p}<1.4 \mathrm{~mm}, \mathrm{~T}=20{ }^{\circ} \mathrm{C}$, $C_{D}=0.92, \bar{\rho}=2.7, R e_{t}=231, G a=192$,

$v_{t}=0.18 \mathrm{~m} / \mathrm{s}, v_{t, B L}=0.21 \mathrm{~m} / \mathrm{s}$, angle $=2.4^{\circ}$,

$-\% v_{t}=17 \%$, path: $S O$
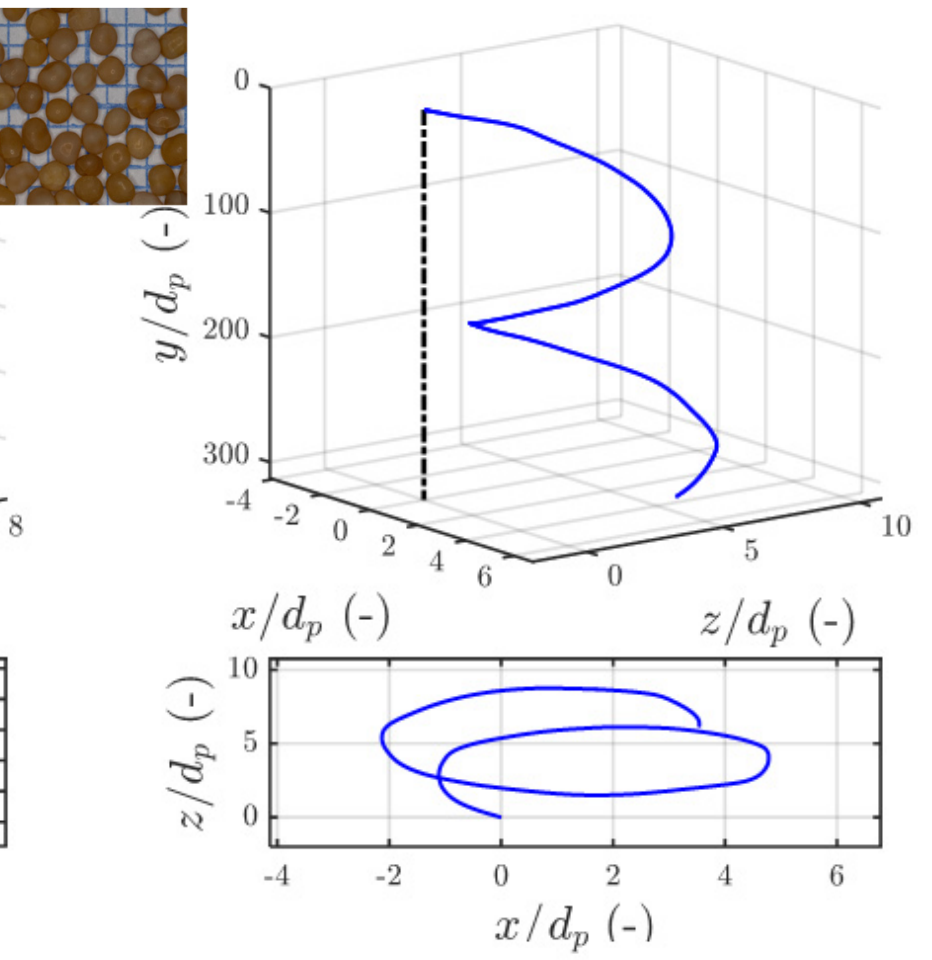

Figure S76 Calcite pellets: $1.25<d_{p}<1.4 \mathrm{~mm}, \mathrm{~T}=20{ }^{\circ} \mathrm{C}$, $C_{D}=0.92, \bar{\rho}=2.7, R e_{t}=232, G a=193$,

$v_{t}=0.18 \mathrm{~m} / \mathrm{s}, v_{t, B L}=0.21 \mathrm{~m} / \mathrm{s}$, angle $=3.0^{\circ}$, $-\% v_{t}=17 \%$, path: $\mathrm{CH}$ 

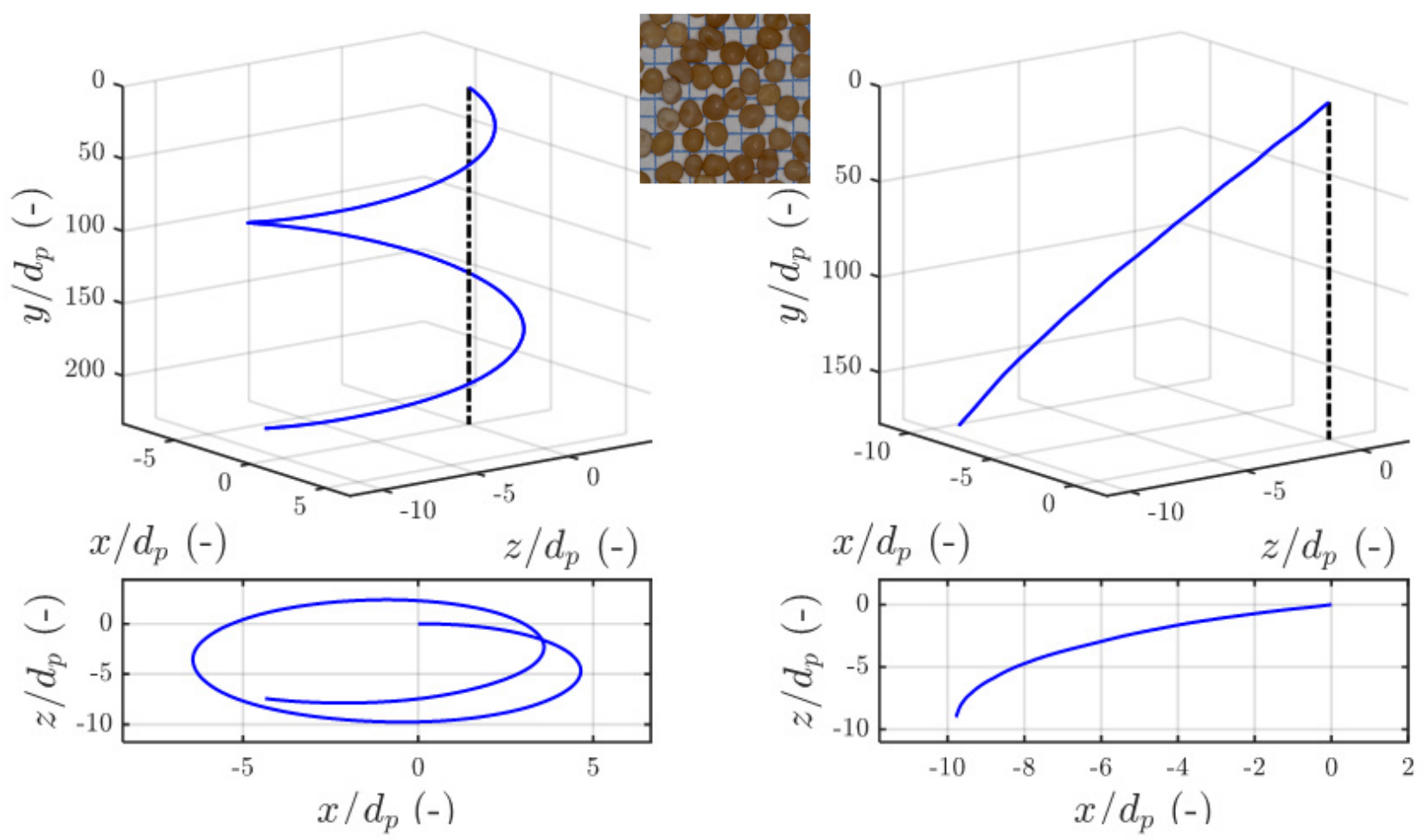

Figure $\boldsymbol{S} 77$ Calcite pellets: $1.12<d_{p}<1.25 \mathrm{~mm}, \mathrm{~T}=21^{\circ} \mathrm{C}$, $C_{D}=1.04, \bar{\rho}=2.7, R e_{t}=189, \mathrm{Ga}=167$,

$v_{t}=0.16 \mathrm{~m} / \mathrm{s}, v_{t, B L}=0.19 \mathrm{~m} / \mathrm{s}$, angle $=5.1^{\circ}$,

$-\% v_{t}=17 \%$, path: $S O$

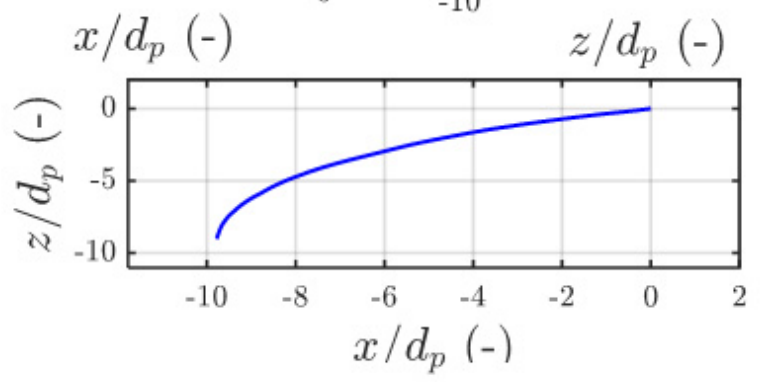

Figure $\mathbf{S 7 8}$ Calcite pellets: $1.12<d_{p}<1.25 \mathrm{~mm}, \mathrm{~T}=21^{\circ} \mathrm{C}$, $C_{D}=0.87, \bar{\rho}=2.7, R e_{t}=207, G a=167$,

$v_{t}=0.17 \mathrm{~m} / \mathrm{s}, v_{t, B L}=0.19 \mathrm{~m} / \mathrm{s}$, angle $=4.7^{\circ}$, $-\% v_{t}=9 \%$, path: $S O$

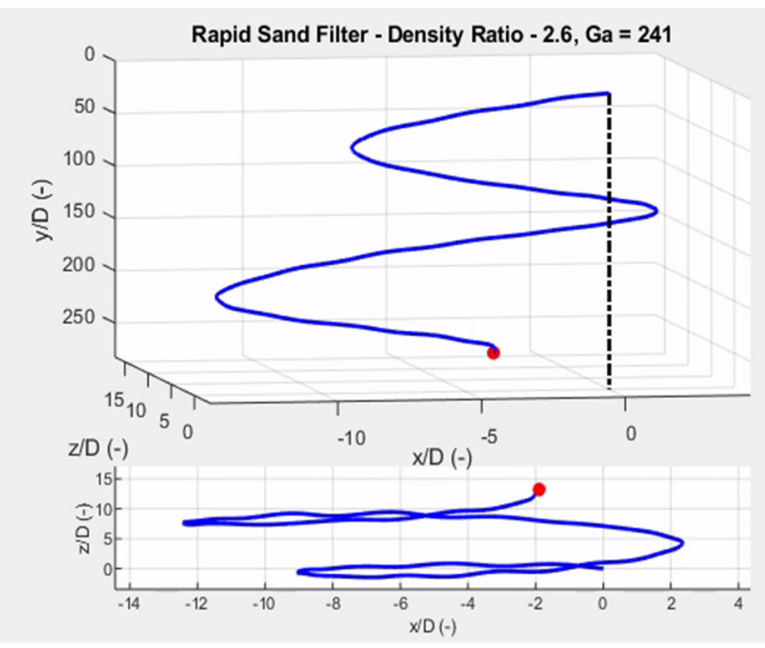

Figure $\mathbf{S 7 9}$ Rapid filter sands: $1.0<d_{p}<2.0 \mathrm{~mm}, T=22$ ${ }^{\circ} \mathrm{C}, C_{D}=1.04, \bar{\rho}=2.6, R_{t}=274, G a=241$, $v_{t}=0.17 \mathrm{~m} / \mathrm{s}, v_{t, B L}=0.19 \mathrm{~m} / \mathrm{s}$, path: $\mathrm{CH}$

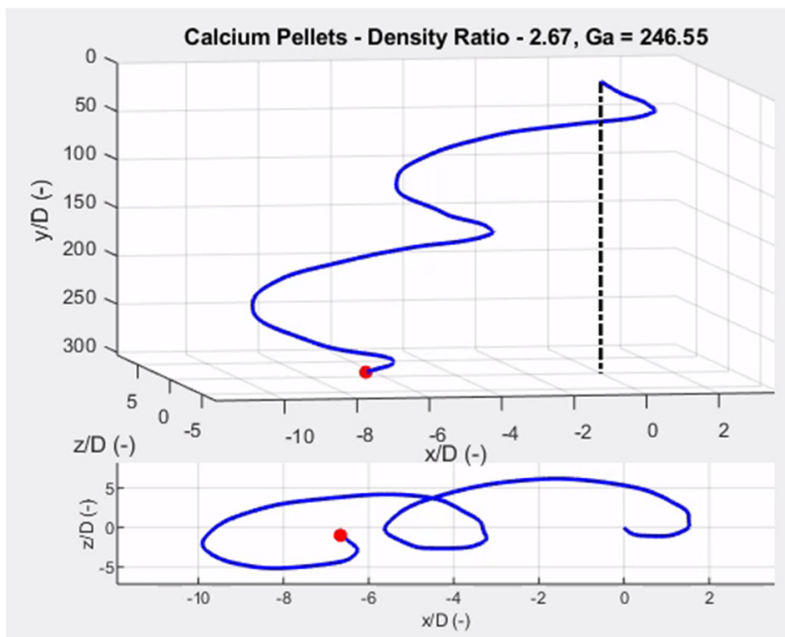

Figure $\mathbf{S 8 0}$ Calcite pellets: $1.4<d_{p}<1.7 \mathrm{~mm}, T=22{ }^{\circ} \mathrm{C}$, $C_{D}=0.62, \bar{\rho}=2.6, R_{t}=361, G a=243$, $v_{t}=0.23 \mathrm{~m} / \mathrm{s}, v_{t, B L}=0.21 \mathrm{~m} / \mathrm{s}$, path: $\mathrm{CH}$ 

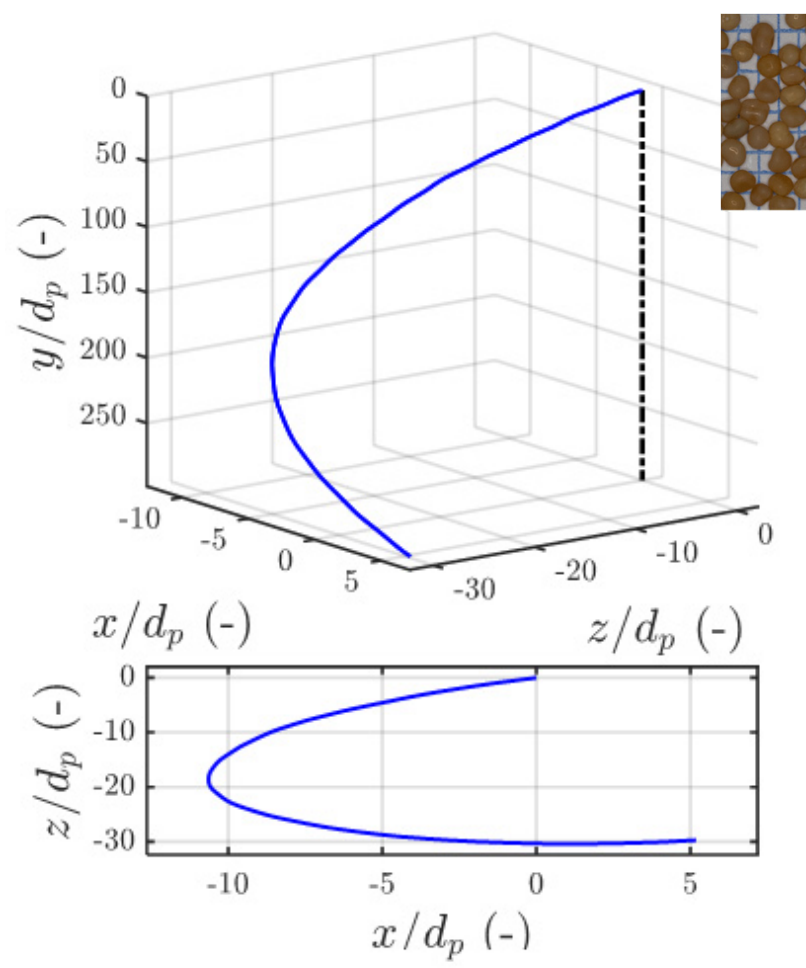

Figure S81 Calcite pellets: $1<d_{p}<1.12 \mathrm{~mm}, \mathrm{~T}=21^{\circ} \mathrm{C}$, $C_{D}=0.93, \bar{\rho}=2.7, R e_{t}=170, G a=142$, $v_{t}=0.16 \mathrm{~m} / \mathrm{s}, v_{t, B L}=0.17 \mathrm{~m} / \mathrm{s}$, angle $=7.8^{\circ}$, $-\% v_{t}=6 \%$, path: SO

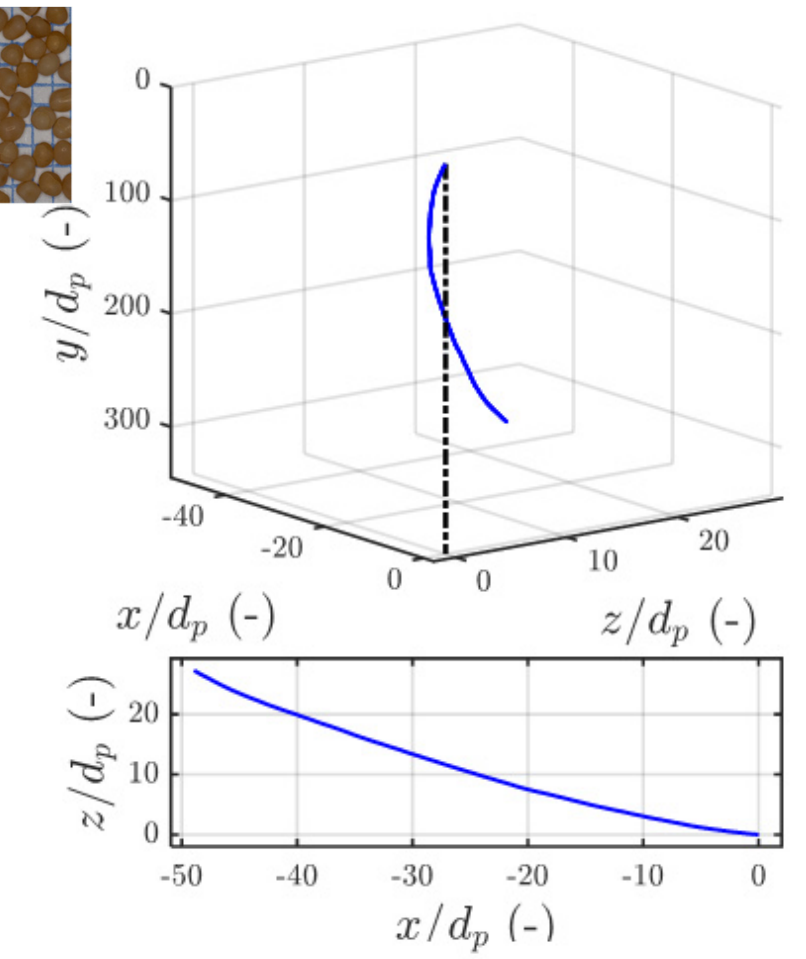

Figure S82 Calcite pellets: $1<d_{p}<1.12 \mathrm{~mm}, T=21{ }^{\circ} \mathrm{C}$, $C_{D}=1.16, \bar{\rho}=2.7, R e_{t}=152, G a=142$, $v_{t}=0.14 \mathrm{~m} / \mathrm{s}, v_{t, B L}=0.17 \mathrm{~m} / \mathrm{s}$, angle $=9.1^{\circ}$, $-\% v_{t}=15 \%$, path: $S O$ 


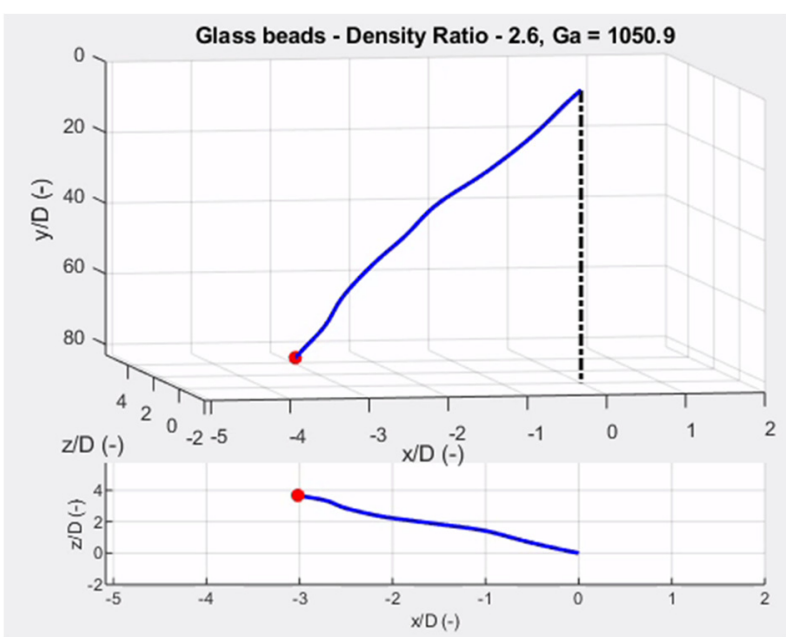

Figure $\mathbf{S 8 3}$ Glass beads: $d_{p}=4.0 \mathrm{~mm}, T=22{ }^{\circ} \mathrm{C}$, $C_{D}=0.43, \bar{\rho}=2.6, R_{t}=1,844, G a=1,051$, $v_{t}=0.44 \mathrm{~m} / \mathrm{s}, v_{t, B L}=0.44 \mathrm{~m} / \mathrm{s}$, path: $\mathrm{CH}$

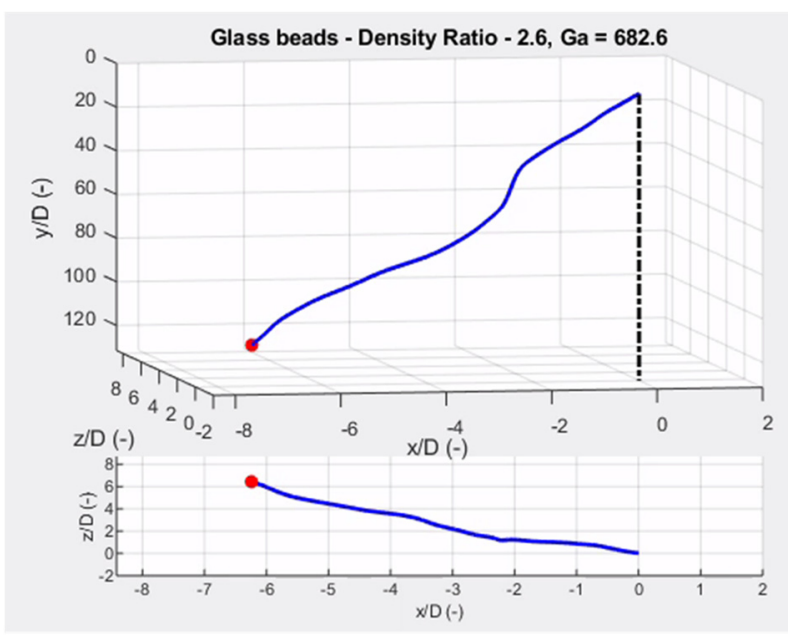

Figure $\mathbf{S 8 5}$ Glass beads: $d_{p}=3.0 \mathrm{~mm}, \mathrm{~T}=22{ }^{\circ} \mathrm{C}$, $C_{D}=0.48, \bar{\rho}=2.6, R e_{t}=1,133, G a=683$, $v_{t}=0.36 \mathrm{~m} / \mathrm{s}, v_{t, B L}=0.36 \mathrm{~m} / \mathrm{s}$, path: $\mathrm{CH}$

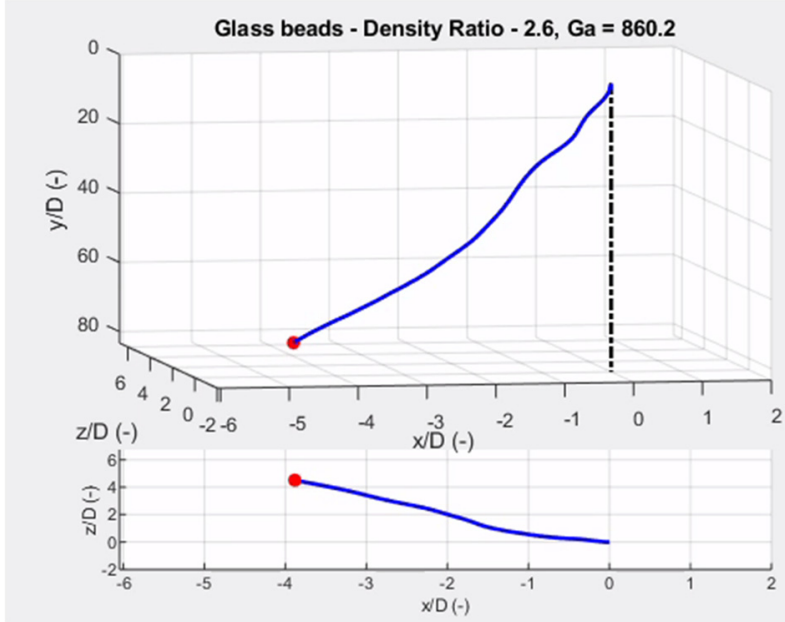

Figure $\mathbf{S 8 4}$ Glass beads: $d_{p}=3.5 \mathrm{~mm}, T=22{ }^{\circ} \mathrm{C}$, $C_{D}=0.44, \bar{\rho}=2.5, \operatorname{Re}_{t}=1,501, G a=840$, $v_{t}=0.41 \mathrm{~m} / \mathrm{s}, v_{t, B L}=0.39 \mathrm{~m} / \mathrm{s}$, path: $\mathrm{CH}$

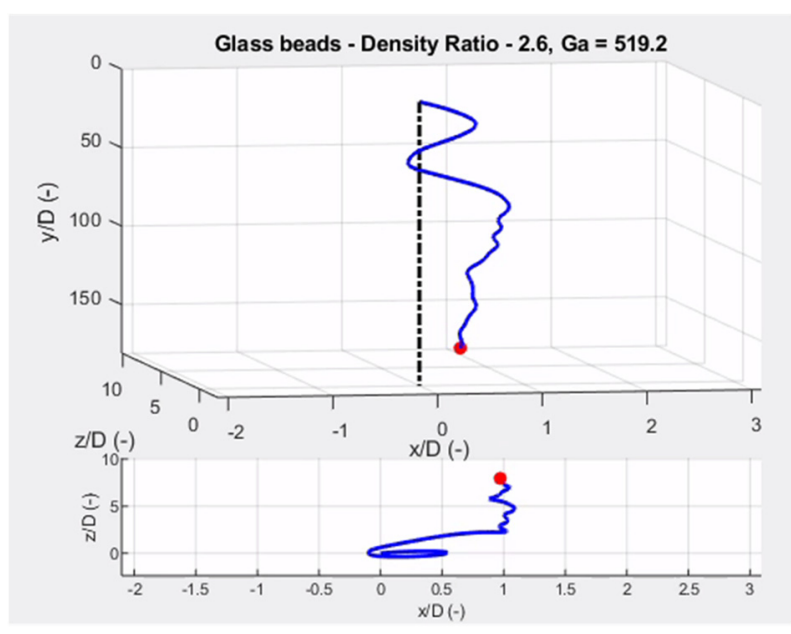

Figure $\mathbf{S 8 6}$ Glass beads: $d_{p}=2.5 \mathrm{~mm}, \mathrm{~T}=22{ }^{\circ} \mathrm{C}$, $C_{D}=0.49, \bar{\rho}=2.5, R e_{t}=858, G a=509$, $v_{t}=0.33 \mathrm{~m} / \mathrm{s}, v_{t, B L}=0.31 \mathrm{~m} / \mathrm{s}$, path: $\mathrm{CH}$ 


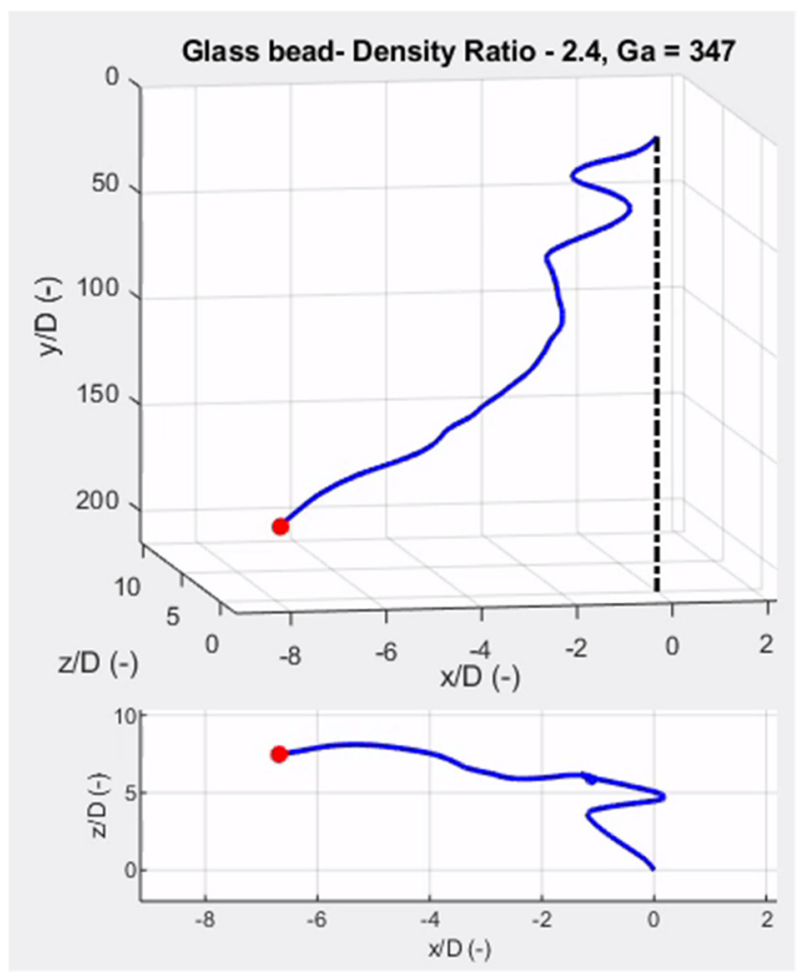

Figure $\mathbf{S 8 7}$ Glass beads: $d_{p}=2.0 \mathrm{~mm}, \mathrm{~T}=22{ }^{\circ} \mathrm{C}$,

$C_{D}=0.47, \bar{\rho}=2.6, R_{t}=584, G a=372, v_{t}=0.2$ $\mathrm{m} / \mathrm{s} 8, v_{t, B L}=0.26 \mathrm{~m} / \mathrm{s}$, path: $\mathrm{CH}$

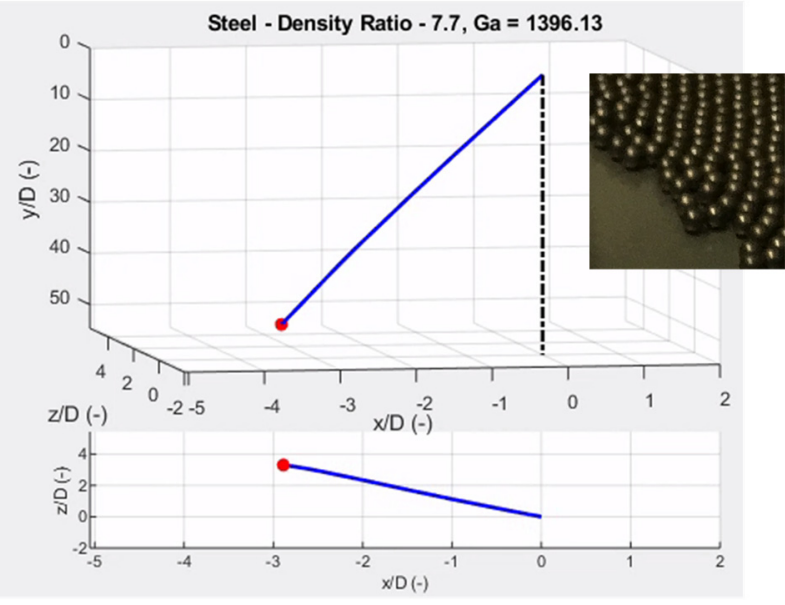

Figure S89 Steel shots: $d_{p}=3.0 \mathrm{~mm}, T=22^{\circ} \mathrm{C}, C_{D}=0.48$, $\bar{\rho}=8.0, R e_{t}=2,602, G a=1,423, v_{t}=0.83 \mathrm{~m} / \mathrm{s}$, $v_{t, B L}=0.81 \mathrm{~m} / \mathrm{s}$, path: $\mathrm{CH}$

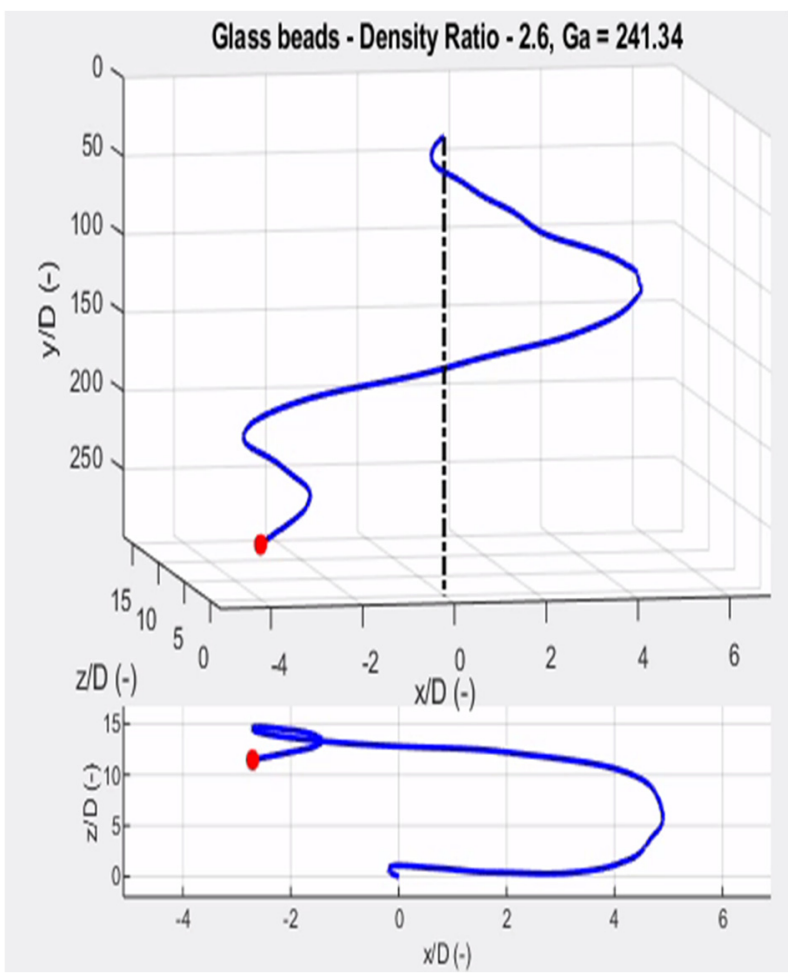

Figure $\mathbf{S 8 8}$ Glass beads: $d_{p}=1.5 \mathrm{~mm}, T=22{ }^{\circ} \mathrm{C}$, $C_{D}=0.66, \bar{\rho}=2.5, R e_{t}=341, G a=236$, $v_{t}=0.22 \mathrm{~m} / \mathrm{s}, v_{t, B L}=0.20 \mathrm{~m} / \mathrm{s}$, path: $\mathrm{CH}$

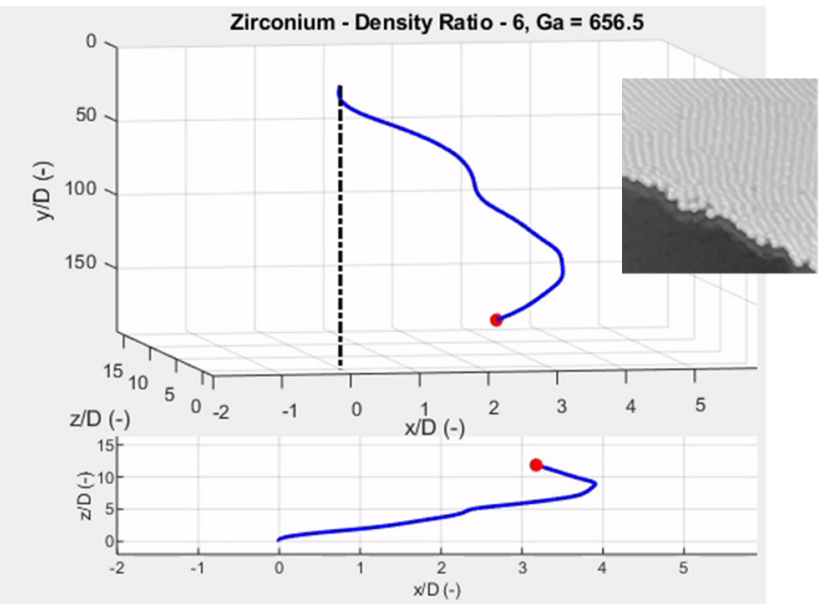

Figure $\mathbf{S 9 0}$ Zirconium balls: $d_{p}=2.0 \mathrm{~mm}, \mathrm{~T}=22{ }^{\circ} \mathrm{C}$, $C_{D}=0.49, \bar{\rho}=6.0, R_{t}=1,141, G a=655$, $v_{t}=0.54 \mathrm{~m} / \mathrm{s}, v_{t, B L}=0.51 \mathrm{~m} / \mathrm{s}$, path: $\mathrm{CH}$ 


\section{S8.2 Path trajectory Table S}

The Table Scontain the divisions of the observed path trajectories according to (Zhou and Dušek, 2015).

Table S22 Path trajectories of examined particles

\begin{tabular}{llll}
\hline Abbreviation & Number & Percentage & Description \\
\hline SO & 2,611 & $71.9 \%$ & Steady oblique \\
LF & 15 & $0.4 \%$ & Steady oblique: low-frequency oscillating \\
HF & 34 & $0.9 \%$ & Steady oblique: high-frequency oscillating \\
CH & 889 & $24.5 \%$ & Three-dimensional chaotic \\
PO & 22 & $0.6 \%$ & Chaotic: high-frequency planar chaotic oblique oscillating \\
QR & 33 & $0.9 \%$ & Chaotic: low-frequency rotating quasi periodic oblique oscillating \\
VP & 25 & $0.7 \%$ & Chaotic: vertical periodic oscillating planar \\
\hline
\end{tabular}

Table S23 Path trajectories of examined calcite and garnet pellets ${ }^{1)}$

\begin{tabular}{llll}
\hline Abbreviation & Number & Percentage & Description \\
\hline SO & 1,459 & $79.7 \%$ & Steady oblique \\
LF & 15 & $0.8 \%$ & Steady oblique: low-frequency oscillating \\
HF & 24 & $1.3 \%$ & Steady oblique: high-frequency oscillating \\
CH & 300 & $16.4 \%$ & Three-dimensional chaotic \\
PO & 0 & $0.0 \%$ & Chaotic: high-frequency planar chaotic oblique oscillating \\
QR & 33 & $1.8 \%$ & Chaotic: low-frequency rotating quasi periodic oblique oscillating \\
VP & 0 & $0.0 \%$ & Chaotic: vertical periodic oscillating planar \\
\hline
\end{tabular}

1) Model derived for spherical particles

Table S24 Path trajectories of examined glass beads and SiLibeads

\begin{tabular}{llll}
\hline Abbreviation & Number & Percentage & Description \\
\hline SO & 584 & $51.2 \%$ & Steady oblique \\
LF & 0 & $0.0 \%$ & Steady oblique: low-frequency oscillating \\
HF & 10 & $0.9 \%$ & Steady oblique: high-frequency oscillating \\
CH & 524 & $46.0 \%$ & Three-dimensional chaotic \\
PO & 22 & $1.9 \%$ & Chaotic: high-frequency planar chaotic oblique oscillating \\
QR & 0 & $0.0 \%$ & Chaotic: low-frequency rotating quasi periodic oblique oscillating \\
VP & 0 & $0.0 \%$ & Chaotic: vertical periodic oscillating planar \\
\hline
\end{tabular}


Table S25 Path trajectories of examined synthetic material (IEX and nylon balls)

\begin{tabular}{llll}
\hline Abbreviation & Number & Percentage & Description \\
\hline SO & 0 & $0.0 \%$ & Steady oblique \\
LF & 0 & $0.0 \%$ & Steady oblique: low-frequency oscillating \\
HF & 0 & $0.0 \%$ & Steady oblique: high-frequency oscillating \\
CH & 24 & $49.0 \%$ & Three-dimensional chaotic \\
PO & 0 & $0.0 \%$ & Chaotic: high-frequency planar chaotic oblique oscillating \\
QR & 0 & $0.0 \%$ & Chaotic: low-frequency rotating quasi periodic oblique oscillating \\
VP & 25 & $51.0 \%$ & Chaotic: vertical periodic oscillating planar \\
\hline
\end{tabular}

Table S26 Path trajectories of examined synthetic material (exotics)

\begin{tabular}{llll}
\hline Abbreviation & Number & Percentage & Description \\
\hline SO & 999 & $84.5 \%$ & Steady oblique \\
LF & 5 & $0.4 \%$ & Steady oblique: low-frequency oscillating \\
HF & 10 & $0.8 \%$ & Steady oblique: high-frequency oscillating \\
CH & 145 & $12.3 \%$ & Three-dimensional chaotic \\
PO & 0 & $0.0 \%$ & Chaotic: high-frequency planar chaotic oblique oscillating \\
QR & 23 & $1.9 \%$ & Chaotic: low-frequency rotating quasi periodic oblique oscillating \\
VP & 0 & $0.0 \%$ & Chaotic: vertical periodic oscillating planar \\
\hline
\end{tabular}

Table S27 Path trajectories of examined synthetic material metal balls (steel shots and zirconium balls)

\begin{tabular}{llll}
\hline Abbreviation & Number & Percentage & Description \\
\hline SO & 7 & $13.2 \%$ & Steady oblique \\
LF & 0 & $0.0 \%$ & Steady oblique: low-frequency oscillating \\
HF & 0 & $0.0 \%$ & Steady oblique: high-frequency oscillating \\
CH & 46 & $86.8 \%$ & Three-dimensional chaotic \\
PO & 0 & $0.0 \%$ & Chaotic: high-frequency planar chaotic oblique oscillating \\
QR & 0 & $0.0 \%$ & Chaotic: low-frequency rotating quasi periodic oblique oscillating \\
VP & 0 & $0.0 \%$ & Chaotic: vertical periodic oscillating planar \\
\hline
\end{tabular}




\section{S8.3 Path trajectory videos}

Videos are shared at: (Kramer et al., 2020c).

DOI: 10.4121/UUID:3FFDFA51-38F0-4188-AEC5-8CD8FC8F1941

Videos: terminal settling experiments in water: path instabilities. Twenty-four path trajectories are shown of grains settling in water in an advanced TU Delft experimental set-up. More technical information can be found here:

http://resolver.tudelft.nl/uuid:cbaf6de1-dcf9-41ab-a5bc-3a4d364bfd45, Path Instabilities of a Rising or Falling Sphere in a

Fluid at Rest - An Experimental Study, authored by (Raaghav, 2019).

The videos were recorded at TU Delft, Department of Mechanical, Maritime and Materials Engineering, the Netherlands.

File: Set 1 - Calcite pellets 1.4-1.7 mm.mp4

File: Set 1 - Glass beads - $1.5 \mathrm{~mm} . \mathrm{mp} 4$

File: Set 1 - Glass beads - $2.0 \mathrm{~mm} . \mathrm{mp} 4$

File: Set 1 - Glass beads - $2.5 \mathrm{~mm} . \mathrm{mp} 4$

File: Set 1 - Glass beads - 3.0 mm.mp4

File: Set 1 - Glass beads - $3.5 \mathrm{~mm} . \mathrm{mp} 4$

File: Set 1 - Glass beads - 4.0 mm.mp4

File: Set 1 - Rapid Filter Sand 1.0-2.0 mm.mp4

File: Set 1 - Steel shots 3.0 mm.mp4

File: Set 1 - Zirconium balls 2.0 mm.mp4

File: Set 2 - Calcite pellets 1-1.12 mm-case-1.mp4 File: Set 2 - Calcite pellets 1-1.12 mm-case-2.mp4 File: Set 2 - Calcite pellets 1-1.12 mm-case-3.mp4 File: Set 2 - Calcite pellets 1.12-1.25 mm-case-1.mp4 File: Set 2 - Calcite pellets 1.12-1.25 mm-case-2.mp4 File: Set 2 - Calcite pellets 1.12-1.25 mm-case-3.mp4 File: Set 2 - Calcite pellets 1.25-1.4 mm-case-1.mp4 File: Set 2 - Calcite pellets 1.25-1.4 mm-case-2.mp4 File: Set 2 - Calcite pellets 1.25-1.4 mm-case-3.mp4 File: Set 2 - Calcite pellets 1.4-1.7 m-case-2.mp4 File: Set 2 - Calcite pellets 1.4-1.7 mm-case-1.mp4 File: Set 2 - Calcite pellets 1.4-1.7 mm-case-3.mp4 File: Set 2 - Calcite pellets 1.7-2 mm-case-1.mp4 File: Set 2 - Calcite pellets 1.7-2 mm-case-2.mp4 File: Set 2 - Calcite pellets 1.7-2 mm-case-3.mp4 File: Set 2 - Calcite pellets 2-2.36 mm-case-1.mp4 File: Set 2 - Calcite pellets 2-2.36 mm-case-2.mp4 File: Set 2 - Calcite pellets 2-2.36 mm-case-3.mp4 File: Set 2 - Calcite pellets 2.36-2.8 mm-case-1.mp4 File: Set 2 - Calcite pellets 2.36-2.8 mm-case-2.mp4 File: Set 2 - Calcite pellets 2.36-2.8 mm-case-3.mp4 File: Set 2 - Calcite pellets $2.8+\mathrm{mm}$-case- $1 . \mathrm{mp} 4$ File: Set 2 - Calcite pellets $2.8+\mathrm{mm}$-case-2.mp4 File: Set 2 - Calcite pellets $2.8+\mathrm{mm}$-case-3.mp4

File: Set 3 - Terminal settling 10mm glass bead.MP4 


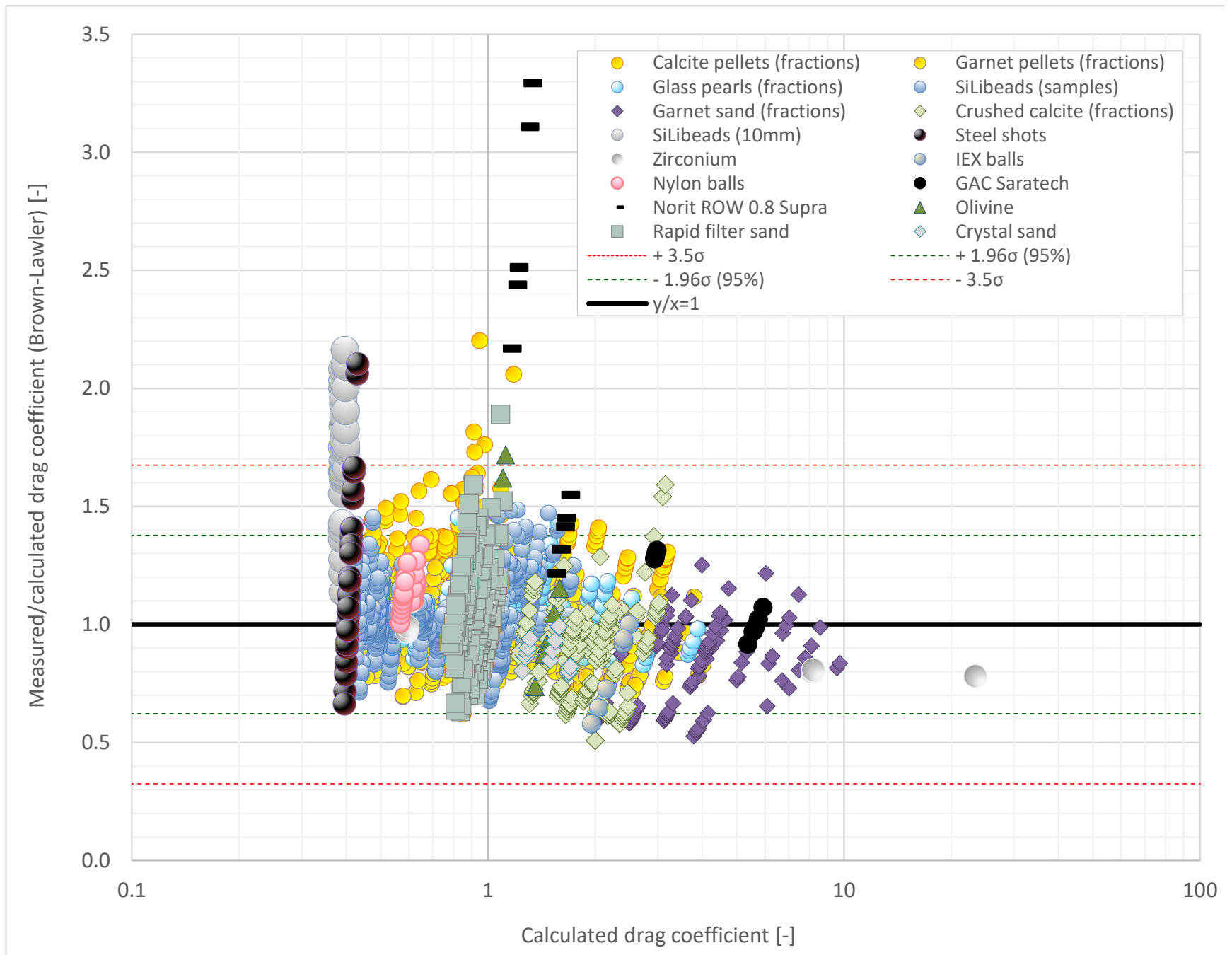

Figure S91 Ratio of measured and calculated terminal settling velocities (Brown and Lawler, 2003) against calculated settling velocity. Statistical probability estimation 95\% $(\mu \pm 1.96 \sigma)$ plot and the $(\mu \pm 3.5 \sigma)$ to show the outliers (mainly garnet) (1.2\%) 42 of 3,629 experimental values 


\section{S10 Drag-Reynolds experimental data spread trends}

\section{S10.1 Observed drag-Reynolds data trends}

When the standard drag curve (dimensionless drag coefficient $C_{D}$ versus terminal Reynolds number $R e_{t}$ ) is plotted using linlin scales instead of log-log scales, the data spread is evidently visible. This can be observed in Figure S92, where fractionated calcite and garnet pellets are plotted. These trends can be explained based on the basic drag coefficient equation.

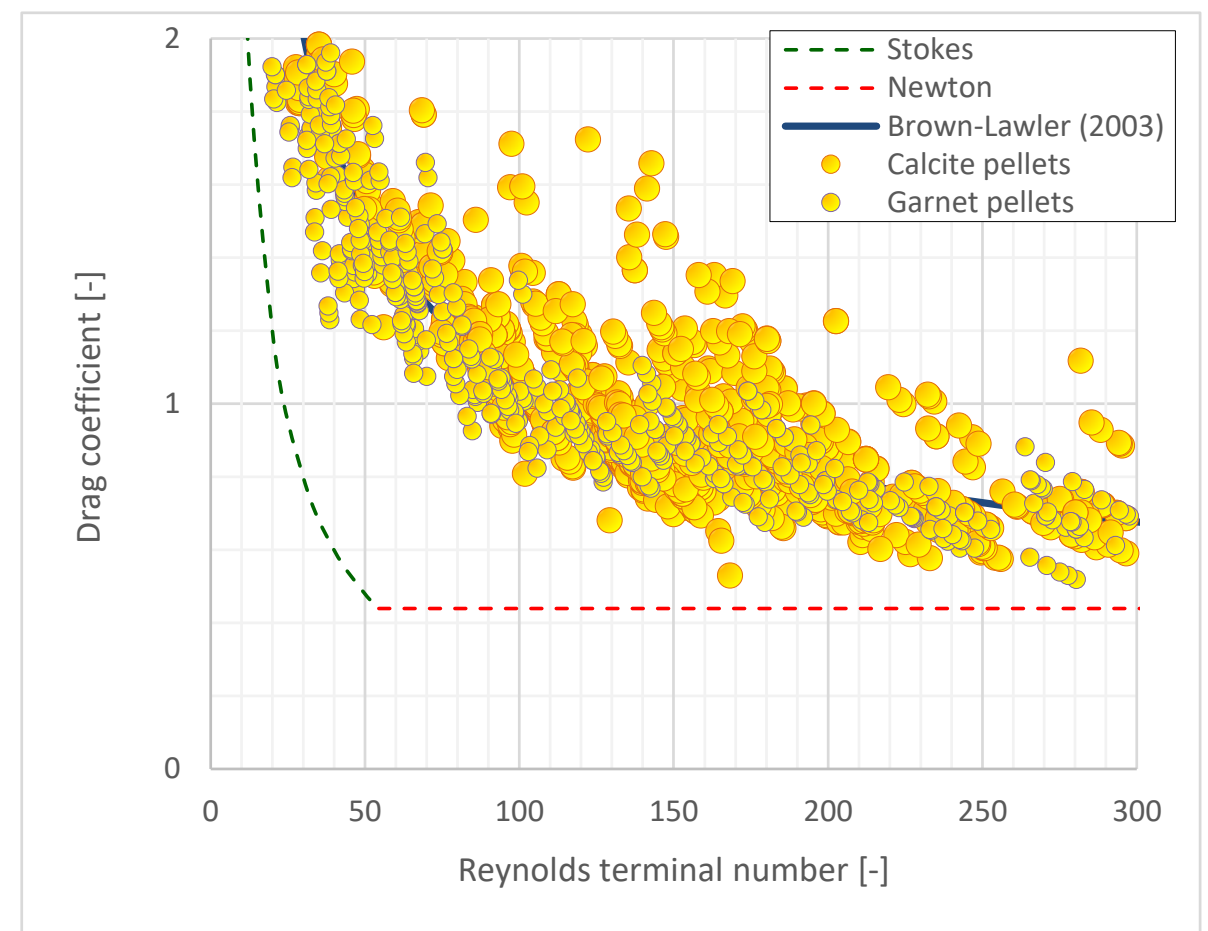

Figure S92 Experimental data spread trends

\section{S10.2 Error made by the laboratory researcher}

To predict the steady terminal velocity of a given particle with a projected surface area $A_{p}$ in the direction of the gravitational field from these correlations, one needs to consider a force balance in which the drag force balances the difference between buoyancy and weight (Gibilaro et al., 1985); (Clift et al., 1978); (Bird et al., 2007); (Brown and Lawler, 2003):

$$
\left(\rho_{p}-\rho_{f}\right) g V=C_{D} A_{p} \frac{1}{2} \rho_{f} v_{t}^{2}
$$

For spherical particles:

$$
\begin{aligned}
& \left(\rho_{p}-\rho_{f}\right) g\left(\frac{\pi}{6} d_{p}^{3}\right)=C_{D}\left(\frac{\pi}{4} d_{p}^{2}\right) \frac{1}{2} \rho_{f} v_{t}^{2} \\
& C_{D} v_{t}^{2}=\frac{\left(\rho_{p}-\rho_{f}\right) g\left(\frac{\pi}{6} d_{p}^{3}\right)}{\left(\frac{\pi}{4} d_{p}^{2}\right) \frac{1}{2} \rho_{f}}
\end{aligned}
$$


$C_{D} v_{t}^{2}=\frac{4}{3} \frac{\left(\rho_{p}-\rho_{f}\right)}{\rho_{f}} g d_{p}$

Let $L$ be the fall distance of a settling particle. The distance can be underpredicted or overpredicted by the error made by the laboratory researcher. Here:

$$
L_{\text {error }}=t_{\text {error }} \bar{v}_{t}
$$

where $t_{\text {error }}$ represents the error made by the laboratory researcher.

$$
\begin{aligned}
& \overline{v_{t}}+v_{t}^{\prime}=\frac{L_{\text {effective }}}{\Delta t_{\text {stopwatch }}}=\frac{L \pm t_{\text {error }} \bar{v}_{t}}{\Delta t_{\text {stopwatch }}} \\
& v_{t}^{\prime} \sim O\left(\frac{t_{\text {error }} \overline{v_{t}}}{\Delta t_{\text {stopwatch }}}\right) \\
& \frac{v_{t}{ }^{\prime}}{\overline{v_{t}}} \sim \pm \frac{t_{\text {error }}}{\Delta t_{\text {stopwatch }}} \\
& \frac{C_{D}{ }^{\prime}}{\overline{C_{D}}} \sim-2 \frac{v_{t}{ }^{\prime}}{\overline{v_{t}}} \sim O\left(\frac{2 t_{\text {error }}}{\Delta t_{\text {stopwatch }}}\right)
\end{aligned}
$$

So:

$$
\frac{C_{D}{ }^{\prime}}{\overline{C_{D}}}[\%] \sim O\left(\frac{2 t_{\text {error }}}{\Delta t_{\text {stopwatch }}}\right)
$$

Let us take an experiment where the total fall error $\Delta t_{\text {stopwatch }}=0.5$ seconds. Consequently, the error made by the laboratory researcher, from start to stop and using Equation (S247), the experiment will yield to an net error of $20 \%$.

Furthermore, for one particular experiment $C_{D} v_{t}^{2}=$ constant, or $C_{D} \sim 1 / v_{t}^{2}$

$$
C_{D} v_{t}^{2}=\frac{4}{3} \frac{\left(\rho_{p}-\rho_{f}\right)}{\rho_{f}} g d_{p}
$$

Now let:

$$
v_{t}=\bar{v}_{t}+v_{t}^{\prime}
$$

where $v_{t}{ }^{\prime}$ is the error / uncertainty $\left(\delta v_{t}\right)$ introduced in velocity due to the student who measures the terminal settling velocity manually using a stopwatch. 


$$
\begin{aligned}
& \overline{C_{D}}+C_{D}{ }^{\prime} \sim \frac{1}{\left(\overline{v_{t}}+v_{t}\right)^{2}} \\
& \overline{C_{D}}+C_{D}{ }^{\prime} \sim \frac{1}{{\overline{v_{t}}}^{2}\left(1+\frac{v_{t}{ }^{\prime}}{\bar{v}_{t}}\right)^{2}} \\
& \overline{C_{D}}+C_{D}{ }^{\prime} \sim \frac{1}{{\overline{v_{t}}}^{2}}\left(1-2 \frac{v_{t}{ }^{\prime}}{\bar{v}_{t}}\right) \\
& \overline{C_{D}}+C_{D}{ }^{\prime} \sim \frac{1}{{\overline{v_{t}}}^{2}}-2 \frac{v_{t}{ }^{\prime}}{\bar{v}_{t}^{3}}
\end{aligned}
$$

Now:

$$
\begin{aligned}
& C_{D}{ }^{\prime} \sim-2 \frac{v_{t}{ }^{\prime}}{{\overline{v_{t}}}^{3}} \\
& \frac{C_{D}{ }^{\prime}}{\overline{C_{D}}} \sim \frac{-2 \frac{v_{t}{ }^{\prime}}{\bar{v}_{t}^{3}}}{\frac{1}{{\overline{v_{t}}}^{2}}} \sim-2 \frac{v_{t}{ }^{\prime}}{\overline{v_{t}}} \\
& \frac{C_{D}{ }^{\prime}}{\overline{C_{D}}} \sim-2 \frac{v_{t}^{\prime}}{\overline{v_{t}}}
\end{aligned}
$$

The slope equals -2 in the SDC and hence for a positive increase in error for $v_{t}{ }^{\prime}$ we see a negative error for $C_{D}{ }^{\prime}$.

\section{S10.3 Correlation error in $C_{D}$ with error in $R e_{t}$}

The force balance at statistically steady state is given by:

$$
C_{D} R e_{t}^{2}=\frac{4}{3} A r
$$

Error analysis in linear approximation (for small errors):

$$
\begin{aligned}
& C_{D}{ }^{\prime} \overline{R e}_{t}^{2}+\left.\overline{C_{D}} 2 \overline{R e}_{t} R e_{t}^{\prime}\right|_{A r=c}=\left.\frac{4}{3} A r^{\prime}\right|_{R e_{t}=c} \\
& C_{D}^{\prime}=\left.\frac{4}{3} \frac{A r^{\prime}}{\overline{R e}_{t}^{2}}\right|_{R e_{t}=c}-\left.2 \overline{C_{D}} \frac{R e_{t}^{\prime}}{\overline{R e}_{t}}\right|_{A r=c}
\end{aligned}
$$


For larger errors, higher order terms in Taylor expansion around base state should be taken into account. $A r^{\prime}$ and $R e_{t}{ }^{\prime}$ will generally not be independent as both depend on $d_{p}$ and $v_{T}$. However, when $R e_{t}{ }^{\prime}$ is solely caused by errors in terminal settling velocity $v_{t}{ }^{\prime}$ and the errors in $A r^{\prime}$ and $R e_{t}{ }^{\prime}$ are independent for multiple experiments on the same particle, we expect that $A r^{\prime}$ will not change. So, it relates to uncertainty in measured parameters on which $A r$ depends, but these parameters do not change when the settling experiment is repeated. In that case, $C_{D}{ }^{\prime}$ is anti-correlated with:

$$
\frac{R e_{t}^{\prime}}{\overline{R e}_{t}}=\frac{v_{t}^{\prime}}{\bar{v}_{t}}
$$

So:

$$
C_{D}^{\prime}=\frac{4}{3} \frac{A r^{\prime}}{\overline{R e}_{t}{ }^{2}}-2 \bar{C}_{D} \frac{v_{t}{ }^{\prime}}{\bar{v}_{t}}
$$

The first right term $\frac{4}{3} \frac{A{ }^{\prime}}{\overline{R e}_{t}{ }^{2}}$ stands for constant upward and downward shift. The second right term $-2 \overline{C_{D}} \frac{v_{t^{\prime}}}{\bar{v}_{t}}$ is the anticorrelation. For $\frac{v_{t}{ }^{\prime}}{\bar{v}_{t}}=-0.2-2$, the spread $\frac{C_{D}{ }^{\prime}}{\overline{C_{D}}}=-0.4-4$, plus the shift from the first term.

\section{S10.4 Direct simplified dependency of the deviation to the variation in particle diameter}

To demonstrate the direct dependency of the deviation to the variation in particle diameter, the simplified drag Equation (S268) can be used. The relative error is given by Equation (S262):

$$
\frac{\Delta C_{D}}{C_{D}}=\beta \frac{\Delta R e_{t}}{R e_{t}} \approx \beta \frac{\Delta d_{p}}{d_{p}}
$$

Coefficient $\beta$ can be retrieved from a $\ln \left(C_{D}\right)$ against $\ln \left(R e_{t}\right)$ and has a value of approximately 0.45 for only calcite pellets. This leads to a similar deviation bandwidth of $\Delta\left(C_{D}\right)=4 \%$ based on $\Delta\left(d_{p}\right)=6 \%$. Since Equation (S268) does not cover the whole range of Reynolds terminal, the straightforward calculated deviation does not coincide exactly with the deviation of $5 \%$. 


\section{S11 Steady state velocity}

Uniform particle motion through a fluid is the result of the action of constant external force such as gravity, buoyancy and the resistance of the fluid to particle motion. In a gravitational field, the motion of spherical particles is governed by Newton's law of motion (Newton, 1726), where all the affecting forces sum up to the net force:

$$
F_{p}=F_{g}+F_{b}+F_{D}
$$

Concerning spherical particles, the force balance becomes the following:

$$
\rho_{p} V_{P} \frac{d v_{t}}{d t}=\rho_{p} V_{P} g-\rho_{f} V_{P} g-C_{D} \frac{1}{2} \rho_{f} v_{t}^{2} A_{p}
$$

The settling velocity of spherical particles in laminar Stokes flow as a function of time is given by (Peker et al., 2008):

$$
v_{t}=\frac{g d_{p}^{2}\left(\rho_{p}-\rho_{f}\right)}{18 \eta}\left(1-e^{\frac{18 \eta}{\rho_{p} d_{p}^{2}} t}\right)
$$

$$
\left(R e_{t}<1\right)
$$

The settling velocity of spherical particles in Newton's regime (Newton, 1726) $\left(C_{D}=0.44\right)$ as a function of time is as follows:

$$
v_{t}=\sqrt{\frac{2 g\left(\rho_{p}-\rho_{f}\right)\left(\frac{\pi}{6} d_{p}{ }^{3}\right)}{\rho_{f}\left(\frac{\pi}{4} d_{p}{ }^{2}\right) C_{D}}} \tanh \left(\sqrt{\frac{\left(\rho_{p}-\rho_{f}\right) g \rho_{f}\left(\frac{\pi}{4} d_{p}{ }^{2}\right) C_{D}}{2 \rho_{p}{ }^{2}\left(\frac{\pi}{6} d_{p}{ }^{3}\right)}} t\right) \quad\left(R e_{t}>1,000\right)
$$

Equations (265) and (266) demonstrate that there are two stages that can be distinguished during the motion of the particle, which are the acceleration and the steady state or constant terminal settling velocity. The terminal settling velocity of a particle is constant when all the forces are in equilibrium:

$$
F_{g}+F_{b}+F_{D}=0
$$

For the transitional regime, no analytic expression is given, so a numerical simulation is required to calculate the settling velocity of spherical particles. In order to be able to estimate the settling velocity, the simplified drag Equation (S268) can be used as given by $(\mathrm{Ku}, 1966)$ and expressed in a general form by (Oka and Anthony, 2003):

$$
C_{D}=\alpha \operatorname{Re}_{t}{ }^{\beta} \quad \quad\left(0.4<R e_{t}<500\right)
$$

in which the Lewis coefficients are $\alpha=10$ and $\beta=-0.5$. Other frequently used values coefficients are (Clark, 2009) $\alpha=18.5$ and $\beta=-0.6$. Combining the drag Equation (S264) and the force balance Equation (S264) gives the following:

$$
\frac{d v_{t}}{d t}=\frac{\left(\rho_{p}-\rho_{f}\right) g}{\rho_{p}}-\frac{1}{2} \alpha\left(\frac{\rho_{f} d_{p}}{\eta}\right)^{\beta} \frac{\left(\frac{\pi}{4} d_{p}^{2}\right) \rho_{f}}{\left(\frac{\pi}{6} d_{p}^{3}\right) \rho_{p}} v_{t}^{2+\beta} \quad \quad\left(0.4<R e_{t}<500\right)
$$




\section{S12 Oblate spheroids}

To investigate the effects of irregularity of non-spherical particles on drag, spheroidal particles were considered to illustrate the effect of particle rotations. Spheroids have been defined by (Ardekani et al., 2016) as ellipsoids with two equal semidiameters, existing in two shapes: prolate (needle-like) and oblate (disc-like). For oblate spheroids, the axis is aligned with the minor diameter of the spheroid, while for prolate spheroids the symmetric axis is aligned with the major diameter. Spheroids are oblate in case $\mathrm{c} / \mathrm{a}<1$ and prolate in case $\mathrm{c} / \mathrm{a}>1$.

For this study, natural particles with a specific average particle diameter were obtained by sieving natural particle mixtures with a wide range of particle sizes. Since sieve openings have square dimensions, there is no clear relation between sieve diameter $\mathrm{d}$ and actual particle size and shape. For spheroidal particles, however, a relation does in fact exist, and therefore the impact on drag could be determined: both prolate and oblate particles can pass a sieve perpendicular to the direction of the gravitational force and therefore $90^{\circ}$ to the orientation of settling sedimentation. The volume for both oblate and prolate particles is $\frac{4}{3} \pi \mathrm{a}^{2} c$. For round spheres, $d_{p}<d$ applies when the expected particles are precisely able to pass a sieve.

Regarding spheroids, however, it is important to realise that those spheroids which were able to pass the sieve will rotate once they have passed this hurdle and will settle in such a way that their bottom surface areas will increase. This is illustrated in Figure S93. The current study examined only those particles which can just pass a sieve. The relation between maximum spheroid size and sieve diameter can be calculated: the equation for oblate spheroids is $d^{2}=a\left(a^{2}+c^{2}\right)$, and for prolate spheroids it is $d=2 a$.

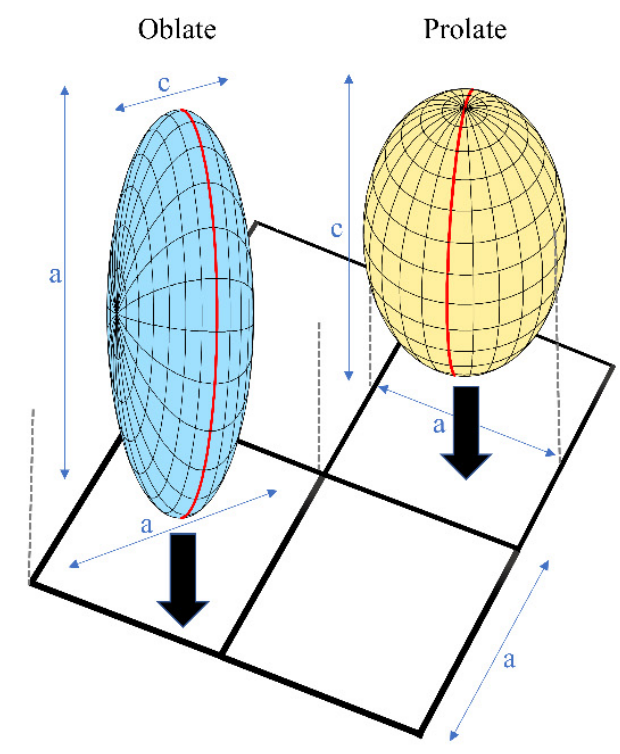

Sieve passage

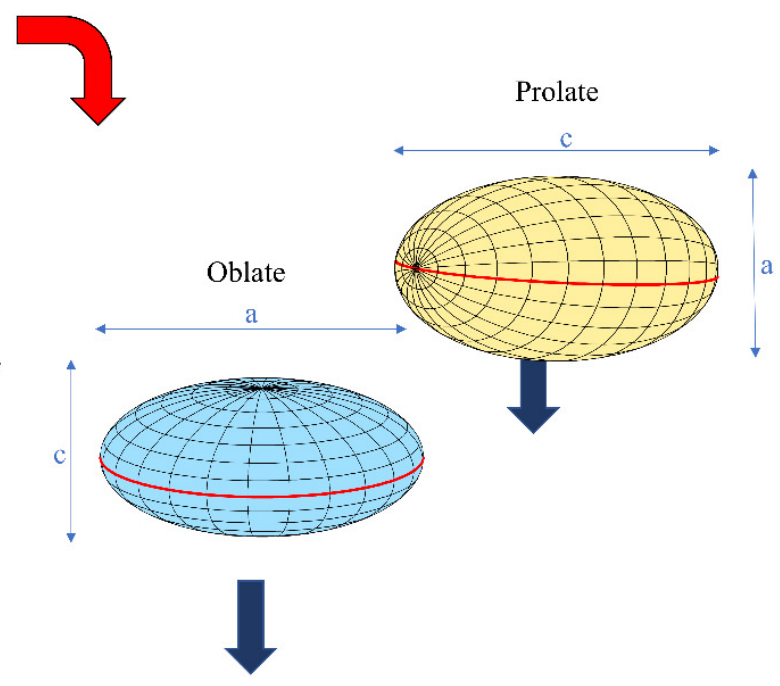

Settling

Figure S93 The assignment of oblate and prolate spheroids upon passing a sieve mesh. Both the oblate and prolate particles change orientation, so that their broad-side becomes perpendicular to the velocity direction.

The force balance for moving spheroids due to gravitational field, buoyancy and weight is slightly different compared to round spheres. The projected surface area regarding oblate spheroids is $\pi a^{2}$ and for prolate spheroids $\pi a c$. The drag coefficient for an oblate spheroid is as follows:

$$
C_{D, o}=\frac{4}{3} \frac{g\left(\rho_{p}-\rho_{f}\right)}{v_{t}^{2} \rho_{f}} c
$$


The drag coefficient for prolate spheroids is:

$$
C_{D, p}=\frac{4}{3} \frac{g\left(\rho_{p}-\rho_{f}\right)}{v_{t}^{2} \rho_{f}} a
$$

In both equations, the drag coefficient is dependent on Reynolds terminal and therefore implicitly dependent on the terminal settling velocity. Because most of our particles are closer to oblate spheroids than to prolate spheroids, in the current study we focused on oblate spheroids only. When the aspect ratio a/c of oblate spheroids is increased, the ratio of volume over the projected surface area decreases. At the same time, the drag coefficient increases with a factor 2 for larger a/c values. This means that it is possible for natural particles with a shape that is similar to that of oblate spheroids to pass a specific sieve to a lower located sieve with a smaller sieve mesh size. In that case, the allocated average sieve diameter for these particles does not coincide.

In Figure S94 the ratio of the volume and area for oblate spheroids has been plotted compared to a perfectly round sphere. The volume ratio for oblate spheroids has been plotted in Figure S95, and Figure S96 shows the effect of the increasing ratio of the volume and area to drag.
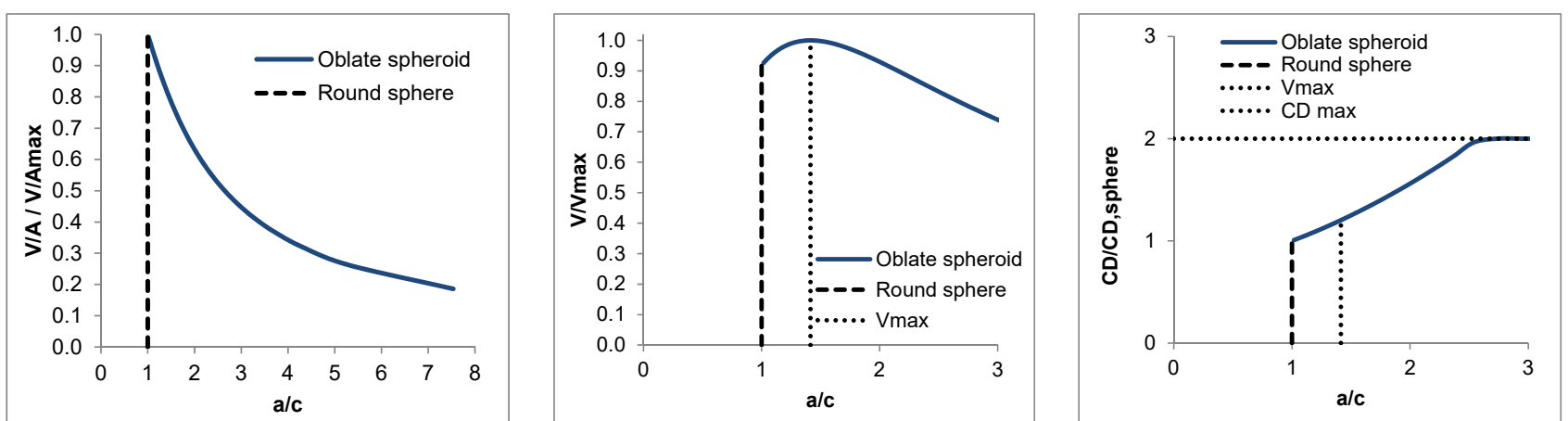

Figure S94 The ratio of volume and area Figure $\mathbf{S 9 5}$ The volume ratio for oblate Figure S96 The drag coefficient $\begin{array}{ll}\text { for oblate spheroids } & \text { spheroid } \\ V / A_{\max }=\frac{2}{3} d, \text { when } a=c, & V_{\max }=\frac{4 \pi d^{3}}{(9 \sqrt{6})}, \text { when } a=d / \sqrt{3}, V= \\ a_{\max }=d / \sqrt{2} & \frac{4}{3} \pi d^{2} c, \text { when } a=c\end{array}$ change for oblate spheroids $C_{D, \text { ref }} / C_{D, \max } \rightarrow 2$, when $a / c \uparrow$

During the terminal settling experiments in which rapid sand filter grains were examined (Figure S32), zig-zag patterns were observed on a regular basis. The vertical motion is caused by gravity and the horizontal movement is caused by particle irregularity according to (Almedeij, 2008) in terms of the influence of the aspect ratio a/c of spheroids. Oblate particles perform a zig-zagging motion, whereas prolate particles rotate around their vertical axis, something which becomes more significant for higher values of the Reynolds number due to instabilities of the vortices in the wake. The influence of particle orientation on the existing drag coefficient is particularly significant with respect to high values for $\operatorname{Ar}_{\text {or }} \operatorname{Re}_{t}$, $(B a g h e r i$ and Bonadonna, 2016). In our study, $20<\mathrm{Ar}<27 \cdot 10^{6}$. In 99.6\% of all measurements, $\mathrm{Ar}>100$. The Archimedes number presents the ratio between gravitational and viscous forces, where $d_{p}$ is the diameter of a sphere with the same volume as the ellipsoidal particle. 


\section{S13 Statistical analysis}

\section{S13.1 Statistical equations}

To compare the experimental data with the prediction models, several well-known statistical methods were used, given an experimentally determined value $y_{\text {exp }}$ and a calculated predicted value $y_{\text {calc }}$. (Haider and Levenspiel, 1989); (Ganser, 1993); (Brown and Lawler, 2003) as well as (Dioguardi et al., 2018) have presented several statistical methods.

The mean average error is given by:

$$
M A E=\frac{1}{n} \sum_{i=1}^{n}\left(\left|y_{\text {calc }, i}-y_{\text {exp }, i}\right|\right)
$$

(Brown and Lawler, 2003) presented the following value $Q$ :

$$
Q=\sum_{i=1}^{n}\left(\log y_{\text {calc }, i}-\log y_{\text {exp }, i}\right)^{2}
$$

And accordingly, the sum of the relative error:

$$
S=\sum_{i=1}^{n}\left(\frac{\left|y_{\text {calc }, i}-y_{\text {exp }, i}\right|}{y_{\text {exp }, i}}\right)
$$

The average relative error:

$$
A R E=\frac{1}{n} \sum_{i=1}^{n} S
$$

or:

$$
A R E=\frac{1}{n} \sum_{i=1}^{n}\left(\frac{\left|y_{\text {calc }, i}-y_{\text {exp }, i}\right|}{y_{\text {exp }, i}}\right)
$$

The sum of squared (relative) errors:

$$
Q_{\text {rel }}=\sum_{i=1}^{n}\left(\frac{y_{\text {calc }, i}-y_{\text {exp }, i}}{y_{\text {exp }, i}}\right)^{2}
$$

The normalised root mean squared error:

$$
\text { NRMSE }=\sqrt{\frac{1}{n} Q_{r e l}}
$$

or: 
$N R M S E=\sqrt{\frac{1}{n} \sum_{i=1}^{n}\left(\frac{y_{c a l c, i}-y_{\text {exp }, i}}{y_{\text {exp }, i}}\right)^{2}}$

The root mean square deviation gives an indication of the average displacement of measured $C_{D}$ values from the correlations.

$$
R M S_{d e v}=\sqrt{\frac{1}{n} Q}
$$

In addition, (Brown and Lawler, 2003) mentions the Pearson correlation Equation (S281).

$$
R=\frac{\sum y_{\text {exp }, i} y_{\text {calc }, i}-\frac{\left(\sum y_{\text {exp }, i} \sum y_{\text {calc }, i}\right)}{N}}{\sqrt{\left(\sum\left(y_{\text {exp }, i}{ }^{2}\right)-\frac{\left(\sum y_{\text {exp }, i}\right)^{2}}{N}\right)\left(\sum\left(y_{\text {calc }, i}{ }^{2}\right)-\frac{\left(\sum y_{\text {calc }, i}\right)^{2}}{N}\right)}}
$$

The pooled or overall weighted variance for all given particle material groups can be calculated with Equation (S282).

$$
\sigma_{t o t}=\sqrt{\frac{\sum_{n=1}^{N}\left(n_{i}-1\right) \sigma_{i}^{2}}{\sum_{i=1}^{N}\left(n_{i}-N\right)}}
$$

To summarise the effect of random errors on the uncertainty of the experimental measurements, a simplified propagation equation by $(\mathrm{Ku}, 1966)$ can be used:

$$
\Delta F=\sqrt{\left(\frac{\partial F}{\partial x}\right)_{y}^{2} \cdot(\Delta x)^{2}+\left(\frac{\partial F}{\partial y}\right)_{y}^{2} \cdot(\Delta y)^{2}+\cdots}
$$

\section{S13.2 Propagated effect of parameter uncertainties on the drag coefficient and terminal Reynolds number}

The summarised propagated effect of errors $(\mathrm{Ku}, 1966)$ on the uncertainty of the experimental measurements is $34 \%$ for $v_{t}$ (Figure Sgiven in the main manuscript), 35\% for the drag coefficient $C_{D}$ (

Figure S97) and $56 \%$ for the terminal Reynolds number (Figure S98).

Table S28 Contribution to error

\begin{tabular}{ll}
\hline Group & Parameter \\
\hline Fluid & Fluid density and viscosity \\
Fluid & Linear thermal expansion \\
Fluid & Path instabilities \\
Particle & Specific particle density \\
Particle & Particle size \\
Particle & Particle shape and orientation \\
Particle & Particle surface roughness \\
External & Gravity \\
External & Wall effects \\
External & Error of measurement \\
\hline
\end{tabular}




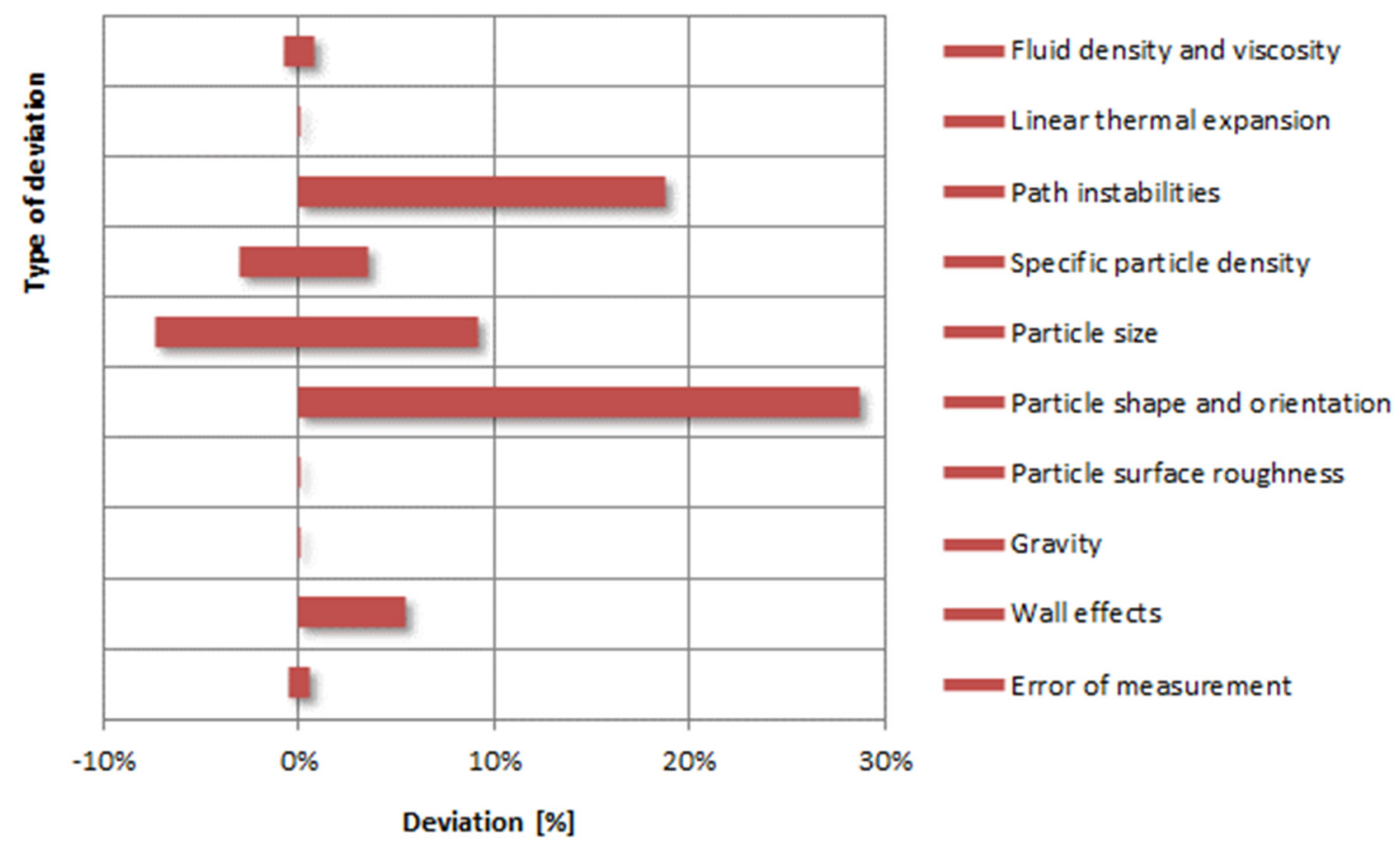

Figure $\mathbf{S 9 7}$ Summarised propagated effect for the drag coefficient resulting from different causes

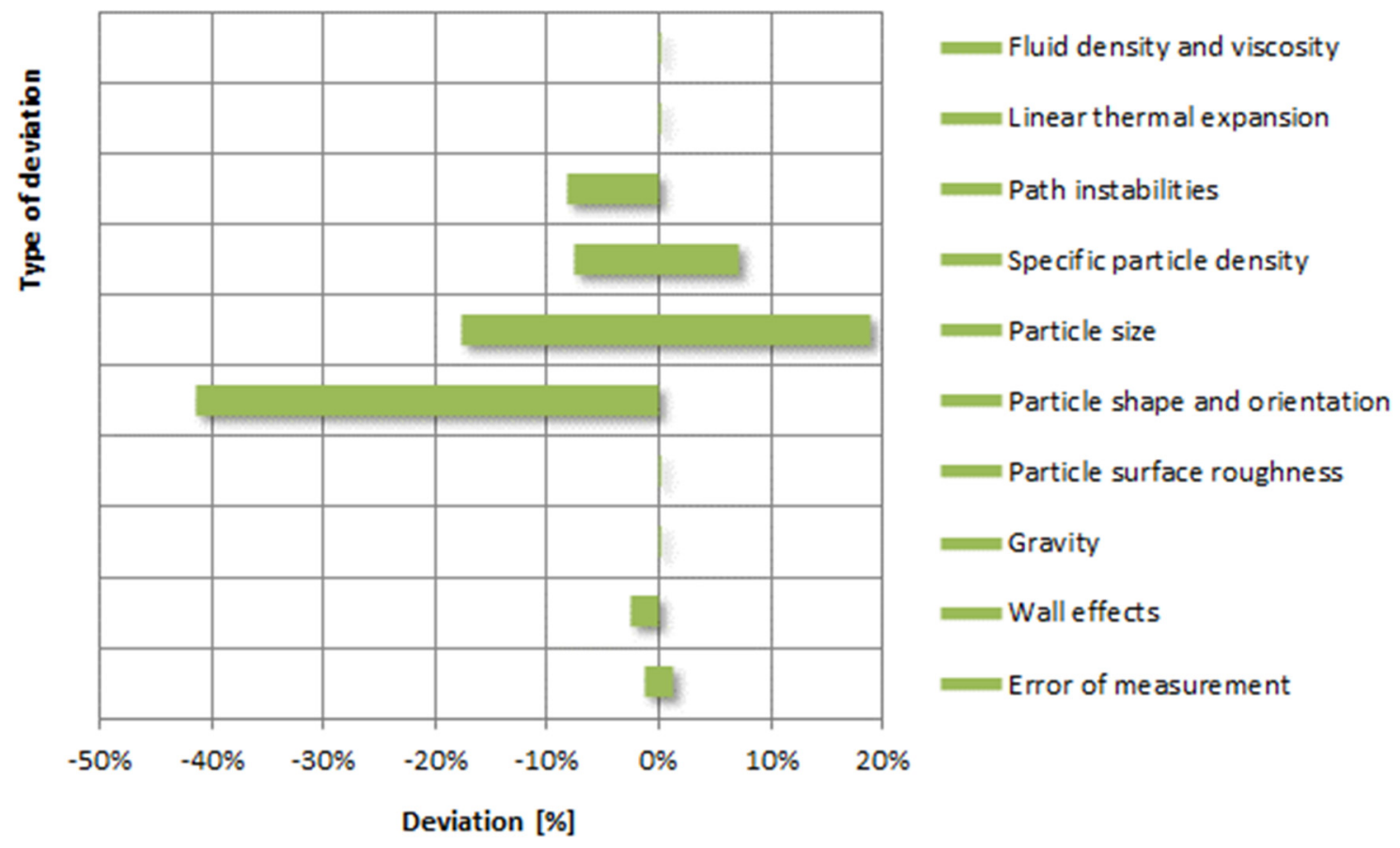

Figure S98 Summarised propagated effect for the terminal Reynolds number resulting from different causes 


\section{S14 Acknowledgments}

Special thanks go to Amir Shokir, Anthony van As, Arjan Brenkman, Auke Smet, Awad Elarbab, Bart de Ruiter, Bart van de Rotten, Cas van Schaik, Cherany Grant, Desmond Lawler, Django Rossa, Edelmar Crichlow, Edo Boek, Ellen Hilhorst, Eveline Glasbergen, Gerard van Hassel, Gerard Visser, Hans Kuipers, Henk-Jan van den Brink, Iloy van Genderen, Jamila Rahman, Jelle van Hattum, Jens van Riel, Jeremy Scheffer, Joop Slaa, Jos Hooft, Jos Vantomme, Jules Engelen, Jurgen Molenaar, Karin Dieters, Kawa Sarwar, Kay Buist, Laetis Kuipers, Lars van Giessen, Leon Kors, Marijn Companjen, Mark Jobse, Mark Joosten, Menno Schaefer, Michel Colin, Peter Wind, Phoebe Berhanu, Rachel Yu, Rens Kuijpers, Richard Rigter, Rick Arends, Roy Duijnmaijer, Sadaf Shirazi, Sam Rutten, Sarah Schaper, Simon van de Kolk, Soufian Chaiat, Soumaya Aissa, Steven Visser, Timo Ponsioen, Victor Shao and Walter Goossens for their contribution to this research. 


\section{S15.1 Symbols}

$a, b, c$ Radius of spheroids [m]

$A, B, C, D$ Coefficients

Ar Archimedes number $A r=g d_{p}{ }^{3} \rho_{f}\left|\rho_{p}-\rho_{f}\right| / \eta^{2}$

$A_{p} \quad$ Particle projected area $\left[\mathrm{m}^{2}\right]$

$A_{s} \quad$ Area of spherical particle $\left[\mathrm{m}^{2}\right]$

$c_{i} \quad$ Coefficients [-]

$C_{D} \quad$ Fluid dynamic drag coefficient $C_{D}=\frac{4}{3} g d_{p}\left|\rho_{p}-\rho_{f}\right| /\left(v_{t}^{2} \rho_{f}\right)$

$\overline{C_{D}} \quad$ Average drag coefficient [-]

$C_{D}{ }^{\prime} \quad$ Error / uncertainty introduced in drag coefficient [-]

D Inner column or cylinder vessel diameter [m]

$d_{g} \quad$ Average seeding material diameter [m]

$d_{i} \quad$ Effective size of a sample where $i$ percentage of particles is smaller than the particular size [m]

$d_{p} \quad$ Effective or average or particle equivalent diameter [m]

$d_{s, i} \quad$ Sieve mesh diameter [m]

error 1.96 times standard deviation

$E_{H, 50}$ Ellipsoid height (cumulative 50\% point) [m]

$E_{L, 50}$ Ellipsoid length (cumulative 50\% point) [m]

$E_{W, 50}$ Ellipsoid width (cumulative 50\% point) [m]

$F_{b} \quad$ Buoyancy force exerted by a fluid that opposes the weight of an immersed object [N]

$F_{D} \quad$ Drag or frictional force of a spherical particle during terminal settling [N]

$F_{g} \quad$ Force by the gravitational field [N]

$F_{p} \quad$ Net force exerting on spherical particle under terminal settling conditions $\quad[\mathrm{N}]$

Ga Galileo number $G a=\sqrt{ }\left(g d_{p}{ }^{3} \rho_{f}\left|\rho_{p}-\rho_{f}\right| / \eta^{2}\right)$

$g \quad$ Local gravitational field of earth equivalent to the free-fall acceleration $\left[\mathrm{m} / \mathrm{s}^{2}\right]$

$k \quad$ Wall effects correction multiplier [-]

$m \quad$ Particle mass [kg]

$n \quad$ Richardson-Zaki coefficient, expansion index [-]

$N \quad$ Total number of particles / total number of experiments [\#]

Re Reynolds number, ratio of inertial forces to viscous forces within a fluid [-]

$R e_{t} \quad$ Reynolds terminal number $R e_{t}=\rho_{f} v_{t} d_{p} / \eta$

$R e_{p} \quad$ Reynolds particle number $R e_{p}=\rho_{f} v_{s} d_{p} / \eta$

Symm Symmetry, the distances between the centre of area to the particle projection borders [-]

UC Non-uniformity coefficient $d_{60} / d_{10}$

$\overline{v_{t}} \quad$ Average terminal settling velocity [m/s]

$v_{t}{ }^{\prime} \quad$ Error / uncertainty introduced in velocity [m/s]

$v_{i} \quad$ Apparent free-falling settling velocity of a particle in an infinite dilution [m/s]

$v_{s} \quad$ Linear superficial velocity or empty tube fluidisation velocity [m/s] 
$v_{t} \quad$ Terminal particle settling velocity

$v_{t, B L} \quad$ Terminal settling velocity according Brown-Lawler [m/s]

$T \quad$ Temperature [ $\left.{ }^{\circ} \mathrm{C}\right]$

$V \quad$ Volume $\left[\mathrm{m}^{3}\right]$

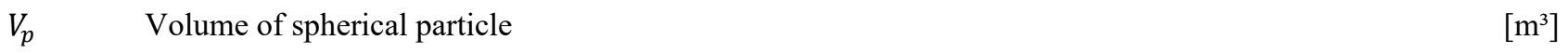

$x \quad$ Average particle diameter between top and bottom sieves [m]

\section{S15.2 Greek symbols}

$\alpha \quad$ Linear heat expansion coefficient

$[\mathrm{m} / \mathrm{mK}]$

$\delta \quad$ Uncertainty

$\varepsilon \quad$ Voidage of the system

$\left[\mathrm{m}^{3} / \mathrm{m}^{3}\right]$

$\eta \quad$ Dynamic fluid viscosity

$[\mathrm{kg} / \mathrm{m} / \mathrm{s}]$

$\lambda \quad$ Ratio between average particle grain diameter and inner column diameter

$\mu \quad$ Statistical mean

$\bar{\rho} \quad$ Specific gravity number, particle to fluid density ratio $\left(\rho_{p} / \rho_{f}\right)$

$\rho_{c} \quad$ Density of calcium carbonate

$\rho_{f} \quad$ Fluid density

$\rho_{g} \quad$ Seeding material density

$\rho_{p} \quad$ Particle density

$v_{T} \quad$ Kinematic fluid viscosity

$\sigma \quad$ Standard deviation

$\phi_{s} \quad$ Shape of diameter correction factor

$\Phi \quad$ Sphericity: ratio between surface area of the volume equivalent sphere and considered particle $\frac{\pi^{\frac{1}{3}}\left(6 V_{p}\right)^{\frac{2}{3}}}{A_{p}}$

$\Phi_{\perp} \quad$ Crosswise sphericity

$\Phi_{\|} \quad$ Lengthwise sphericity

$\Psi \quad$ Particle shape descriptor

$\Xi \quad$ Circularity calculated from the perimeter $\mathrm{P}$ and area $\mathrm{A}$ of the particle projection $\sqrt{\frac{4 \pi A_{p}}{P^{2}}}$

\section{S15.3 Subscripts, superscripts and abbreviations}
ARE Average relative error
BL Brown and Lawler
c Calcium carbonate
calc Calculated value
$\mathrm{CH}$ Three-dimensional chaotic (regime path trajectory)
exp Experimental value
f $\quad$ Fluid properties
FG Fair and Geyer
g Garnet 
GAC Granular activated carbon

HF Steady oblique: high-frequency oscillating (regime path trajectory)

i Index number

IEX Anionic exchange polymer resins

LF Steady oblique: low-frequency oscillating (regime path trajectory)

MAE Mean average error

$\max$ Maximum

NRMSE Normalized root mean square error

p Particle properties

P\&ID Piping and instrumentation diagram

PO Chaotic: high-frequency planar chaotic oblique oscillating (regime path trajectory)

QR Chaotic: low-frequency rotating quasi periodic oblique oscillating (regime path trajectory)

$\mathrm{R}^{2} \quad$ Correlation coefficient

ref Reference value

SDC Standard drag curve

SO Steady oblique (regime path trajectory)

t Terminal settling conditions

TDS Total dissolved solids

tot Total or overall

UC Uniformity coefficient (sieve analysis)

VP Chaotic: vertical periodic oscillating planar (regime path trajectory) 


\section{S16 Bibliography}

Akgiray, O. and Soyer, E.: An evaluation of expansion equations for fluidized solid-liquid systems, Journal of Water Supply: Research and Technology - AQUA, 55(7-8), 517-525, https://doi.org/10.2166/aqua.2006.040, 2006.

Almedeij, J.: Drag coefficient of flow around a sphere: Matching asymptotically the wide trend, Powder Technology, 186(3), 218-223, https://doi.org/10.1016/j.powtec.2007.12.006, 2008.

Anthony, J. W., Bideaux, R. A., Bladh, K. W. and Nichols, M. C.: Calcite, handbook of mineralogy", V, borates, carbonates, sulfates, mineral data publishing, 1st ed., edited by U. Chantilly, VA, Mineralogical Society of America., 2003.

Ardekani, M. N., Costa, P., Breugem, W. P. and Brandt, L.: Numerical study of the sedimentation of spheroidal particles, International Journal of Multiphase Flow, 87, 16-34, https://doi.org/10.1016/j.ijmultiphaseflow.2016.08.005, 2016.

Arsenijević, Z. L., Grbavčić, Ž. B., Garić-Grulović, R. v. and Bošković-Vragolović, N. M.: Wall effects on the velocities of a single sphere settling in a stagnant and counter-current fluid and rising in a co-current fluid, Powder Technology, 203(2), 237 242, https://doi.org/10.1016/j.powtec.2010.05.013, 2010.

Bagheri, G. and Bonadonna, C.: On the drag of freely falling non-spherical particles, Powder Technology, 301, 526-544, https://doi.org/10.1016/j.powtec.2016.06.015, 2016.

Baldock, T. E., Tomkins, M. R., Nielsen, P. and Hughes, M. G.: Settling velocity of sediments at high concentrations, Coastal Engineering, 51(1), 91-100, https://doi.org/10.1016/j.coastaleng.2003.12.004, 2004.

Bird, R. B., Stewart, W. E. and Lightfoot, E. N.: Transport phenomena, revised, 2nd ed., John Wiley \& Sons, Inc. International, New-York., 2007.

Breakey, D. E. S., Vaezi, G. , F., Masliyah, J. H. and Sanders, R. S.: Side-view-only determination of drag coefficient and settling velocity for non-spherical particles, Powder Technology, 339, 182-191, https://doi.org/10.1016/j.powtec.2018.07.056, 2018.

Brown, P. P. and Lawler, D. F.: Sphere drag and settling velocity revisited, Journal of Environmental Engineering, 129(3), 222-231, https://doi.org/10.1061/(ASCE)0733-9372(2003)129:3(222), 2003.

Cheng, N. S.: Comparison of formulas for drag coefficient and settling velocity of spherical particles, Powder Technology, 189(3), 395-398, https://doi.org/10.1016/j.powtec.2008.07.006, 2009.

Chhabra, R. P., Agarwal, S. and Chaudhary, K.: A note on wall effect on the terminal falling velocity of a sphere in quiescent Newtonian media in cylindrical tubes, Powder Technology, 129(1-3), 53-58, https://doi.org/10.1016/S0032-5910(02)00164X, 2003.

Chien, S. F.: Settling velocity of irregularly shaped particles, SPE Drilling \& Completion, 9(4), 281-289, https://doi.org/10.1016/0148-9062(95)92494-3, 1994.

Civan, F.: Critical modification to the Vogel-Tammann-Fulcher equation for temperature effect on the density of water, Industrial and Engineering Chemistry Research, 46(17), 5810-5814, https://doi.org/10.1021/ie070714j, 2007.

Clark, M. M.: Transport modeling for environmental engineers and scientists, 2nd ed., John Wiley \& Sons Ltd., New York., 2009.

Clift, R. and Gauvin, W. H.: Motion of entrained particles in gas streams, Canadian Journal of Chemical Engineering, 49(4), 439-448, https://doi.org/https://doi.org/10.1002/cjee.5450490403, 1971.

Clift, R., Grace, J. R. and Weber, M. E.: Bubbles, drops, and particles, Academic Press., San Diego, San Diego, C.A., 1978.

Dallavalle, J. M.: Micromeritics - the technology of fine particles, 2nd ed., Pitman Publishing Ltd, London., 1948.

Davis, M.: Water and wastewater engineering - Design principles and practice, 1st ed., McGraw-Hill, New-York., 2010.

Dharmarajah, A. H.: Effect of particle shape on prediction of velocity-voidage relationship in fluidized solid-liquid systems, Ames, 1982.

van Dijk, J. C. and Wilms, D. A.: Water treatment without waste material-fundamentals and state of the art of pellet softening, Journal Water Supply: Research and Technology: AQUA, 40(5), 263-280, 1991. 
Dioguardi, F. and Mele, D.: A new shape dependent drag correlation formula for non-spherical rough particles. Experiments and results, Powder Technology, 277, 222-230, https://doi.org/10.1016/j.powtec.2015.02.062, 2015.

Dioguardi, F., Mele, D. and Dellino, P.: A new one-equation model of fluid drag for irregularly shaped particles valid over a wide range of Reynolds number, Journal of Geophysical Research: Solid Earth, 123(1), 144-156, https://doi.org/10.1002/2017JB014926, 2018.

Fair, G. M., Geyer, J. C. and Okun, D. A.: Elements of water supply and waste water disposal, 1st ed., John Wiley \& Sons., New York., 1954.

di Felice, R.: Review article number 47: Of hydrodynamics of liquid fluidisation, Chemical Engineering Science, 50(8), 12131245, https://doi.org/10.1016/0009-2509(95)98838-6, 1995.

di Felice, R. and Gibilaro, L. G.: Wall effects for the pressure drop in fixed beds, Chemical Engineering Science, 59(14), 30373040, https://doi.org/10.1016/j.ces.2004.03.030, 2004.

Ferreira, T. and Rasband, W.: ImageJ user guide., 2012.

Fidleris, V. and Whitmore, R. L.: Experimental determination of the wall effect for spheres falling axially in cylindrical vessels, British Journal of Applied Physics, 12(9), 490-494, https://doi.org/10.1088/0508-3443/12/9/311, 2002.

Flemmer, R. L. C. and Banks, C. L.: On the drag coefficient of a sphere, Powder Technology, 48(3), 217-221, https://doi.org/10.1016/0032-5910(86)80044-4, 1986.

Ganser, G. H.: A rational approach to drag prediction of spherical and nonspherical particles, Powder Technology, 77(2), 143152, https://doi.org/10.1016/0032-5910(93)80051-B, 1993.

Geldart, D.: Types of gas fluidization, Powder Technology, 7(5), 285-292, https://doi.org/10.1016/0032-5910(73)80037-3, 1973.

Gibilaro, L. G., di Felice, R., Waldram, S. P. and Foscolo, P. U.: Generalized friction factor and drag coefficient correlations for fluid particle interactions, Chemical Engineering Science, 40(10), 1817-1823, 1985.

Graf, W. H.: Hydraulics of sediment transport, 1st ed., Water Resources Publications, LLC, Colorado., 1984.

Graveland, A., van Dijk, J. C., de Moel, P. J. and Oomen, J. H. C. M.: Developments in water softening by means of pellet reactors, Journal AWWA - American Water Works Association, 75(12), 619-625, 1983.

Haberman, W. L. and Sayre, R. M.: Motion of rigid and fluid spheres in stationary and moving liquids inside cylindrical tubes., 1958.

Haider, A. and Levenspiel, O.: Drag coefficients and terminal velocity of spherical and nonspherical particles, Powder Technology, 58(1), 63-70, 1989.

Hofman, J. A. M., Kramer, O. J. I., van der Hoek, J. P., Nederlof, M. M. and Groenendijk, M.: Twenty years of experience with central softening in the Netherlands, water quality, environmental benefits and costs, in Water 21, International Symposium on Health Aspects of Calcium and Magnesium in Drinking Water, MD. International Life Sciences Institute, 2426 April 2006, pp. 1-8, Washington, DC., USA, http://resolver.tudelft.nl/uuid:e43683de-0270-46c4-b736-c3ddfe9973e6, 2007.

Hölzer, A. and Sommerfeld, M.: New simple correlation formula for the drag coefficient of non-spherical particles, Powder Technology, 184(3), 361-365, https://doi.org/10.1016/j.powtec.2007.08.021, 2008.

Jenny, M., Dušek, J. and Bouchet, G.: Instabilities and transition of a sphere falling or ascending freely in a Newtonian fluid, Journal of Fluid Mechanics, 508(508), 201-239, https://doi.org/10.1017/S0022112004009164, 2004.

Khan, A. R. and Richardson, J. F.: The resistance to motion of a solid sphere in a fluid, Chemical Engineering Communications, 62(1-6), 135-150, https://doi.org/10.1080/00986448708912056, 1987.

Khan, A. R. and Richardson, J. F.: Fluid-particle interactions and flow characteristics of fluidized beds and settling suspensions of spherical particles, Chemical Engineering Communications, 78(1), 111-130, https://doi.org/10.1080/00986448908940189, 1989. 
Koza, J. R.: Genetic programming: on the programming of computers by means of natural selection complex adaptive systems. papers2://publication/uuid/5DADD85F-EE2F-42E1-8BF8-2CC6959C4FA0, 1992.

Kramer, O. J. I., de Moel, P. J., Baars, E. T., van Vugt, W. H., Padding, J. T. and van der Hoek, J. P.: Improvement of the Richardson-Zaki liquid-solid fluidisation model on the basis of hydraulics, Powder Technology, 343, 465-478, https://doi.org/10.1016/j.powtec.2018.11.018, 2019.

Kramer, O. J. I., de Moel, P. J., Padding, J. T., Baars, E. T., el Hasadi, Y. M. F., Boek, E. S. and van der Hoek, J. P.: Accurate voidage prediction in fluidisation systems for full-scale drinking water pellet softening reactors using data driven models, Journal of Water Process Engineering, 37(101481), 1-15, https://doi.org/10.1016/j.jwpe.2020.101481, 2020a.

Kramer, O. J. I., Padding, J. T., Vugt, W. H. van, Moel, P. J. de, Baars, E. T., Boek, E. S. and van der Hoek, J. P.: Improvement of voidage prediction in liquid-solid fluidized beds by inclusion of the Froude number in effective drag relations, International Journal of Multiphase Flow, 127(101481), 1-13, https://doi.org/10.1016/j.ijmultiphaseflow.2020.103261, 2020 b.

Kramer, O. J. I., Raaghav, S. K. R. and Breugem, W. P.: Videos of terminal settling experiments in water: path instabilities [Data set] 4TU.Centre for Research Data, 4TU.Centre for Research Data, The Netherlands., 2020c.

$\mathrm{Ku}, \mathrm{H}$. H.: Notes on the use of propagation of error formulas, Journal of Research of the National Bureau of Standards, Section C: Engineering and Instrumentation, 70C(4), 263, https://doi.org/10.6028/jres.070C.025, 1966.

Leith, D.: Drag on nonspherical objects, Aerosol Science and Technology, 6(2), 153-161, https://doi.org/10.1080/02786828708959128, 1987.

Loeffler, A. L.: Mechanism of hindered settling and fluidization, Ames, 1953.

Morrison, F. A.: An introduction to fluid mechanics, Cambridge University Press, New York., 2013.

do Nascimento, O. L., Reay, D. A. and Zivkovic, V.: Influence of surface forces and wall effects on the minimum fluidization velocity of liquid-solid micro-fluidized beds, Powder Technology, 304, 55-62, https://doi.org/10.1016/j.powtec.2016.05.013, 2016.

NEN-EN 933-2: Tests for geometrical properties of aggregates - Part 2: Determination of particle size distribution - Test sieves, nominal sizes of apertures, Dutch norm, ICS-code 91.100.15, 93.080.20., n.d.

Newton, I.: Philosophiæ naturalis principia mathematica, 3rd ed., University Press, Cambridge., 1726.

Nutonian: Eureqa, Genetic Programming and Evolvable Machines, www.nutonian.com/research/, 2019.

Oka, S. and Anthony, E. J.: Fluidized bed combustion, CRC Press, New-York., 2003.

Peker, S. M., Helvaci, Ş. Ş., Yener, B., Ikizler, B. and Alparslan, A.: Solid-liquid two phase flow, 1st ed., Elsevier Science, Amsterdam., 2008.

Perry, R. H. and Green, D. W.: Perry's chemical engineers' handbook, 50th ed., McGraw-Hill Int., New York., 2007.

Raaghav, S. K. R.: Path instabilities of a rising or falling sphere in a fluid at rest - an experimental study, Delft http://resolver.tudelft.nl/uuid:cbaf6de1-dcf9-41ab-a5bc-3a4d364bfd45, 2019.

Rao, A., Curtis, J. S., Hancock, B. C. and Wassgren, C.: The effect of column diameter and bed height on minimum fluidization velocity, AIChE Journal - American Institute of Chemical Engineers, 56(9), 2304-2311, https://doi.org/10.1002/aic.12161, 2010.

Retsch-Technology: Operating instructions / Manual particle size analysis system: Camsizer., 2007.

Richardson, J. F. and Zaki, W. N.: Sedimentation and fluidisation: part I, Transactions of the Institution of Chemical Engineers, 32, 35-53, https://doi.org/10.1016/S0263-8762(97)80006-8, 1954.

Rumble, J.: Handbook of chemistry and physics, 101th ed., CRC Press, New York., 2019.

van Schagen, K. M., Rietveld, L. C., Babuška, R. and Kramer, O. J. I.: Model-based operational constraints for fluidised bed crystallisation, Water Research, 42(1-2), 327-337, https://doi.org/10.1016/j.watres.2007.07.019, 2008. 
Schetters, M. J. A., van der Hoek, J. P., Kramer, O. J. I., Kors, L. J., Palmen, L. J., Hofs, B. and Koppers, H.: Circular economy in drinking water treatment: re-use of ground pellets as seeding material in the pellet softening process, Water Science and Technology, 71(4), 479-486, https://doi.org/10.2166/wst.2014.494, 2015.

Schiller, L. and Naumann, A.: Über die grundlegenden berechnungen bei der schwerkraftaufbereitung, Zeitschrift des Vereines Deutscher Ingenieure, 29, 318-320, 1933.

Serway, R. A. and Jewett, J. W.: Physics for scientists and engineers, 9th ed., edited by Brooks/Cole Cengage Learning, Physical Sciences: Mary Finch, Boston., 2014.

Song, X., Xu, Z., Li, G., Pang, Z. and Zhu, Z.: A new model for predicting drag coefficient and settling velocity of spherical and non-spherical particle in Newtonian fluid, Powder Technology, 321, 242-250, https://doi.org/10.1016/j.powtec.2017.08.017, 2017.

Stevens, B. L., Lewis, F. L. and Johnson, E. N.: Aircraft control and simulation - dynamics, controls design, and autonomous systems, 3rd ed., John Wiley \& Sons, New York., 2016.

Stokes, G. G.: On the effect of the internal friction of fluids on the motion of pendulums, Transactions of the Cambridge Philosophical Society, 4, 1-8, https://doi.org/10.1017/CBO9780511702242.005, 1850.

Tang, C., Jørgensen Hedegaard, M., Lopato, L. and Albrechtsen, H. J.: Softening of drinking water by the pellet reactor Effects of influent water composition on calcium carbonate pellet characteristics, Science of the Total Environment, 652, 538548, https://doi.org/10.1016/j.scitotenv.2018.10.157, 2019.

Terfous, A., Hazzab, A. and Ghenaim, A.: Predicting the drag coefficient and settling velocity of spherical particles, Powder Technology, 239, 12-20, https://doi.org/10.1016/j.powtec.2013.01.052, 2013.

Turton, R. and Levenspiel, O.: A short note on the drag correlation for spheres, Powder Technology, 47(1), 83-86, https://doi.org/10.1016/0032-5910(86)80012-2, 1986.

van der Veen, C. and Graveland, A.: Central softening by crystallization in a fluidized-bed process, Journal American Water Works Association, 80(6), 51-58, https://doi.org/10.1002/j.1551-8833.1988.tb03053.x, 1988.

Vogel, H.: Das temperatur-abhängigkeitsgesetz der viskosität von flüssigkeiten, Zeitschrift für Physik, 22, 645-646, 1921.

Whiten, W. J. and Özer, C. E.: New relation for the computation of settling velocities and diameters of spheres, Mineral Processing and Extractive Metallurgy Review, 36(2), 92-102, https://doi.org/10.1080/08827508.2014.885904, 2015.

Wu, W., Asce, M., Wang, S. S. Y. and Asce, F.: Formulas for sediment porosity and settling velocity, Journal of Hydraulic Engineering, 132(8), 858-862, https://doi.org/10.1061/(ASCE)0733-9429(2006)132:8(858), 2006.

Yang, W. C.: Handbook of fluidization and fluid-particle systems, 1st ed., CRC Press, New-York., 2003.

Zhou, W. and Dušek, J.: Chaotic states and order in the chaos of the paths of freely falling and ascending spheres, International Journal of Multiphase Flow, 75, 205-223, https://doi.org/10.1016/j.ijmultiphaseflow.2015.05.010, 2015. 


\section{S17.1 Experimental data (this work)}

Experimental data of terminal settling measurements (all 3629 values)

Geldart's particle classification (Geldart, 1973):

- Group A: 'aeratable S' particles

- Group B: $\quad$ is called 'sandlike' or 'bubbly' particles

- Group C: materials are 'cohesive', or very 'fine powders'

- Group D: $\quad$ is called 'spoutable S' and the materials are either very large or very dense

Regime path trajectories (Zhou and Dušek, 2015):

- $\mathrm{SO}$ :

- $\mathrm{CH}$ :

- LF:

- HF:

- PO:

- QR:

- VP:
Steady oblique

Three-dimensional chaotic

Steady oblique: low-frequency oscillating

Steady oblique: high-frequency oscillating

Chaotic: high-frequency planar chaotic oblique oscillating

Chaotic: low-frequency rotating quasi periodic oblique oscillating

Chaotic: vertical periodic oscillating planar

\begin{tabular}{|c|c|c|c|c|c|c|c|c|c|c|c|c|}
\hline \multirow{2}{*}{$\begin{array}{l}\text { Nr. } \\
\text { coefficient } \\
{[\#]}\end{array}$} & \multirow{2}{*}{$\begin{array}{l}\text { Grain type plain } \\
{[-]}\end{array}$} & \multirow{2}{*}{$\begin{array}{l}\text { Geldarts } \\
\text { group } \\
{[-]}\end{array}$} & \multirow{2}{*}{$\begin{array}{l}\text { Regime } \\
\text { type } \\
{[-]}\end{array}$} & \multirow{2}{*}{$\begin{array}{l}\mathrm{L} \\
\text { Fall } \\
\text { length } \\
{[\mathrm{m}]}\end{array}$} & \multirow{2}{*}{$\begin{array}{l}\mathrm{dp}, 1 \\
\text { Particle size } \\
\text { sieve } 1 \\
{[\mathrm{~mm}]}\end{array}$} & \multirow{2}{*}{$\begin{array}{l}\mathrm{dp}, 2 \\
\text { sieve } 2 \\
{[\mathrm{~mm}]}\end{array}$} & \multirow{2}{*}{$\begin{array}{l}\mathrm{T} \\
\text { Temperature } \\
{\left[{ }^{\circ} \mathrm{C}\right]}\end{array}$} & \multirow{2}{*}{$\begin{array}{l}\text { D } \\
\text { Inner column } \\
\text { diameter } \\
{[\mathrm{m}]}\end{array}$} & \multirow{2}{*}{$\begin{array}{l}\mathrm{t} \\
\text { Settling } \\
\text { time } \\
{[\mathrm{s}]}\end{array}$} & \multirow{2}{*}{$\begin{array}{l}\text { rho_p } \\
\text { Specific } \\
\text { particle density } \\
{\left[\mathrm{kg} / \mathrm{m}^{3}\right]}\end{array}$} & $\begin{array}{l}\text { phi } \\
\text { Sphericity } \\
\text { yCamsizer }\end{array}$ & $\begin{array}{l}\text { alpha } \\
\text { Linear thermal } \\
\text { expansion }\end{array}$ \\
\hline & & & & & & & & & & & {$[-]$} & {$[\mathrm{m} / \mathrm{Km}] \cdot 10^{-6}$} \\
\hline 1 & GAC (Norit ROW 0.8 Supra) & $\mathrm{B}$ & SO & 1.50 & 0.89 & 3.15 & 3.3 & 0.057 & 35.85 & 1,200 & 0.74 & 6.0 \\
\hline 2 & GAC (Norit ROW 0.8 Supra) & B & SO & 1.50 & 0.89 & 3.15 & 3.3 & 0.057 & 33.70 & 1,200 & 0.74 & 6.0 \\
\hline 3 & GAC (Norit ROW 0.8 Supra) & B & SO & 1.50 & 0.89 & 3.15 & 3.3 & 0.057 & 34.29 & 1,200 & 0.74 & 6.0 \\
\hline 4 & GAC (Norit ROW 0.8 Supra) & B & SO & 1.50 & 0.89 & 3.15 & 3.3 & 0.057 & 32.10 & 1,200 & 0.74 & 6.0 \\
\hline 5 & GAC (Norit ROW 0.8 Supra) & B & So & 1.50 & 0.89 & 3.15 & 3.3 & 0.057 & 30.40 & 1,200 & 0.74 & 6.0 \\
\hline 6 & GAC (Norit ROW 0.8 Supra) & B & SO & 1.50 & 0.89 & 3.15 & 32.0 & 0.057 & 37.38 & 1,200 & 0.74 & 6.0 \\
\hline 7 & GAC (Norit ROW 0.8 Supra) & B & SO & 1.50 & 0.89 & 3.15 & 32.0 & 0.057 & 34.61 & 1,200 & 0.74 & 6.0 \\
\hline 8 & GAC (Norit ROW 0.8 Supra) & B & SO & 1.50 & 0.89 & 3.15 & 32.0 & 0.057 & 38.12 & 1,200 & 0.74 & 6.0 \\
\hline 9 & GAC (Norit ROW 0.8 Supra) & $\mathrm{B}$ & SO & 1.50 & 0.89 & 3.15 & 32.0 & 0.057 & 43.88 & 1,200 & 0.74 & 6.0 \\
\hline 10 & GAC (Norit ROW 0.8 Supra) & B & SO & 1.50 & 0.89 & 3.15 & 32.0 & 0.057 & 45.61 & 1,200 & 0.74 & 6.0 \\
\hline 11 & GAC (Saratech) & A & SO & 1.50 & 0.44 & 0.51 & 3.3 & 0.057 & 73.80 & 1,420 & 0.96 & 6.0 \\
\hline 12 & GAC (Saratech) & A & SO & 1.50 & 0.44 & 0.51 & 3.3 & 0.057 & 68.00 & 1,420 & 0.96 & 6.0 \\
\hline 13 & GAC (Saratech) & A & SO & 1.50 & 0.44 & 0.51 & 3.3 & 0.057 & 65.00 & 1,420 & 0.96 & 6.0 \\
\hline 14 & GAC (Saratech) & A & SO & 1.50 & 0.44 & 0.51 & 3.3 & 0.057 & 71.00 & 1,420 & 0.96 & 6.0 \\
\hline 15 & GAC (Saratech) & A & SO & 1.50 & 0.44 & 0.51 & 3.3 & 0.057 & 69.00 & 1,420 & 0.96 & 6.0 \\
\hline 16 & GAC (Saratech) & A & SO & 1.50 & 0.44 & 0.51 & 32.0 & 0.057 & 56.30 & 1,420 & 0.96 & 6.0 \\
\hline 17 & GAC (Saratech) & A & SO & 1.50 & 0.44 & 0.51 & 32.0 & 0.057 & 56.40 & 1,420 & 0.96 & 6.0 \\
\hline 18 & GAC (Saratech) & A & SO & 1.50 & 0.44 & 0.51 & 32.0 & 0.057 & 56.65 & 1,420 & 0.96 & 6.0 \\
\hline 19 & GAC (Saratech) & A & SO & 1.50 & 0.44 & 0.51 & 32.0 & 0.057 & 57.20 & 1,420 & 0.96 & 6.0 \\
\hline 20 & GAC (Saratech) & A & SO & 1.50 & 0.44 & 0.51 & 32.0 & 0.057 & 57.48 & 1,420 & 0.96 & 6.0 \\
\hline 21 & Synthetic material (IEX) & A & SO & 2.00 & 0.90 & 1.18 & 17.1 & 0.057 & 84.62 & 1,090 & 0.95 & 75.0 \\
\hline 22 & Synthetic material (IEX) & A & SO & 2.00 & 0.90 & 1.18 & 17.1 & 0.057 & 89.91 & 1,090 & 0.95 & 75.0 \\
\hline 23 & Synthetic material (IEX) & A & SO & 2.00 & 0.90 & 1.18 & 17.4 & 0.057 & 65.53 & 1,090 & 0.95 & 75.0 \\
\hline 24 & Synthetic material (IEX) & A & SO & 2.00 & 0.90 & 1.18 & 17.4 & 0.057 & 60.60 & 1,090 & 0.95 & 75.0 \\
\hline 25 & Synthetic material (IEX) & A & So & 2.00 & 0.90 & 1.18 & 17.4 & 0.057 & 71.22 & 1,090 & 0.95 & 75.0 \\
\hline 26 & Synthetic material (IEX) & A & SO & 2.00 & 0.90 & 1.18 & 17.4 & 0.057 & 85.57 & 1,090 & 0.95 & 75.0 \\
\hline 27 & Olivine & B & SO & 1.50 & 0.50 & 0.90 & 3.3 & 0.057 & 14.00 & 3,400 & 0.85 & 10.0 \\
\hline 28 & Olivine & B & SO & 1.50 & 0.50 & 0.90 & 3.3 & 0.057 & 12.00 & 3,400 & 0.85 & 10.0 \\
\hline 29 & Olivine & B & SO & 1.50 & 0.50 & 0.90 & 3.3 & 0.057 & 11.60 & 3,400 & 0.85 & 10.0 \\
\hline 30 & Olivine & B & so & 1.50 & 0.50 & 0.90 & 3.3 & 0.057 & 10.33 & 3,400 & 0.85 & 10.0 \\
\hline 31 & Olivine & B & SO & 1.50 & 0.50 & 0.90 & 3.3 & 0.057 & 13.10 & 3,400 & 0.85 & 10.0 \\
\hline 32 & Olivine & B & SO & 1.50 & 0.50 & 0.90 & 32.0 & 0.057 & 8.60 & 3,400 & 0.85 & 10.0 \\
\hline 33 & Olivine & B & SO & 1.50 & 0.50 & 0.90 & 32.0 & 0.057 & 10.01 & 3,400 & 0.85 & 10.0 \\
\hline 34 & Olivine & $\mathrm{B}$ & SO & 1.50 & 0.50 & 0.90 & 32.0 & 0.057 & 10.93 & 3,400 & 0.85 & 10.0 \\
\hline 35 & Olivine & B & SO & 1.50 & 0.50 & 0.90 & 32.0 & 0.057 & 13.76 & 3,400 & 0.85 & 10.0 \\
\hline 36 & Olivine & B & SO & 1.50 & 0.50 & 0.90 & 32.0 & 0.057 & 14.30 & 3,400 & 0.85 & 10.0 \\
\hline 37 & Garnet (distortion layer) & B & SO & 1.21 & 0.30 & 0.36 & 14.8 & 0.125 & 21.29 & 3,026 & 0.85 & 7.0 \\
\hline 38 & Garnet (distortion layer) & B & So & 1.21 & 0.30 & 0.36 & 14.8 & 0.125 & 23.50 & 3,026 & 0.85 & 7.0 \\
\hline 39 & Garnet (distortion layer) & B & SO & 1.21 & 0.30 & 0.36 & 14.8 & 0.125 & 21.50 & 3,026 & 0.85 & 7.0 \\
\hline 40 & Garnet (distortion layer) & B & SO & 1.21 & 0.30 & 0.36 & 14.8 & 0.125 & 22.31 & 3,026 & 0.85 & 7.0 \\
\hline 41 & Garnet (distortion layer) & B & so & 1.21 & 0.30 & 0.36 & 14.8 & 0.125 & 22.47 & 3,026 & 0.85 & 7.0 \\
\hline 42 & Mined calcite (IT) & B & SO & 2.00 & 0.43 & 0.56 & 2.9 & 0.057 & 20.10 & 2,575 & 0.84 & 25.0 \\
\hline 43 & Mined calcite (IT) & B & SO & 2.00 & 0.43 & 0.56 & 2.9 & 0.057 & 20.13 & 2,575 & 0.84 & 25.0 \\
\hline 44 & Mined calcite (IT) & B & SO & 2.00 & 0.43 & 0.56 & 2.9 & 0.057 & 23.02 & 2,575 & 0.84 & 25.0 \\
\hline 45 & Mined calcite (IT) & B & SO & 2.00 & 0.43 & 0.56 & 2.9 & 0.057 & 23.03 & 2,575 & 0.84 & 25.0 \\
\hline 46 & Mined calcite (IT) & B & so & 2.00 & 0.43 & 0.56 & 2.9 & 0.057 & 23.22 & 2,575 & 0.84 & 25.0 \\
\hline 47 & Mined calcite (IT) & B & SO & 2.00 & 0.43 & 0.56 & 2.9 & 0.057 & 23.47 & 2,575 & 0.84 & 25.0 \\
\hline 48 & Mined calcite (IT) & B & So & 2.00 & 0.43 & 0.56 & 11.1 & 0.057 & 22.29 & 2,575 & 0.84 & 25.0 \\
\hline 49 & Mined calcite (IT) & B & SO & 2.00 & 0.43 & 0.56 & 11.1 & 0.057 & 22.53 & 2,575 & 0.84 & 25.0 \\
\hline 50 & Mined calcite (IT) & $\mathrm{B}$ & SO & 2.00 & 0.43 & 0.56 & 11.1 & 0.057 & 22.91 & 2,575 & 0.84 & 25.0 \\
\hline 51 & Mined calcite (IT) & B & So & 2.00 & 0.43 & 0.56 & 11.1 & 0.057 & 23.15 & 2,575 & 0.84 & 25.0 \\
\hline 52 & Mined calcite (IT) & B & SO & 2.00 & 0.43 & 0.56 & 11.1 & 0.057 & 23.28 & 2,575 & 0.84 & 25.0 \\
\hline 53 & Mined calcite (IT) & B & SO & 2.00 & 0.43 & 0.56 & 11.1 & 0.057 & 23.41 & 2,575 & 0.84 & 25.0 \\
\hline 54 & Mined calcite (IT) & B & SO & 2.00 & 0.43 & 0.56 & 11.1 & 0.057 & 24.06 & 2,575 & 0.84 & 25.0 \\
\hline 55 & Mined calcite (IT) & B & SO & 2.00 & 0.43 & 0.56 & 11.1 & 0.057 & 24.10 & 2,575 & 0.84 & 25.0 \\
\hline 56 & Mined calcite (IT) & B & SO & 2.00 & 0.43 & 0.56 & 20.2 & 0.057 & 20.88 & 2,575 & 0.84 & 25.0 \\
\hline 57 & Mined calcite (IT) & B & SO & 2.00 & 0.43 & 0.56 & 20.2 & 0.057 & 21.15 & 2,575 & 0.84 & 25.0 \\
\hline 58 & Mined calcite (IT) & B & SO & 2.00 & 0.43 & 0.56 & 20.2 & 0.057 & 21.18 & 2,575 & 0.84 & 25.0 \\
\hline 59 & Mined calcite (IT) & B & SO & 2.00 & 0.43 & 0.56 & 20.2 & 0.057 & 21.22 & 2,575 & 0.84 & 25.0 \\
\hline 60 & Mined calcite (IT) & B & SO & 2.00 & 0.43 & 0.56 & 20.2 & 0.057 & 21.50 & 2,575 & 0.84 & 25.0 \\
\hline 61 & Mined calcite (IT) & B & SO & 2.00 & 0.43 & 0.56 & 20.2 & 0.057 & 21.57 & 2,575 & 0.84 & 25.0 \\
\hline 62 & Mined calcite (IT) & B & SO & 2.00 & 0.43 & 0.56 & 20.2 & 0.057 & 21.69 & 2,575 & 0.84 & 25.0 \\
\hline
\end{tabular}




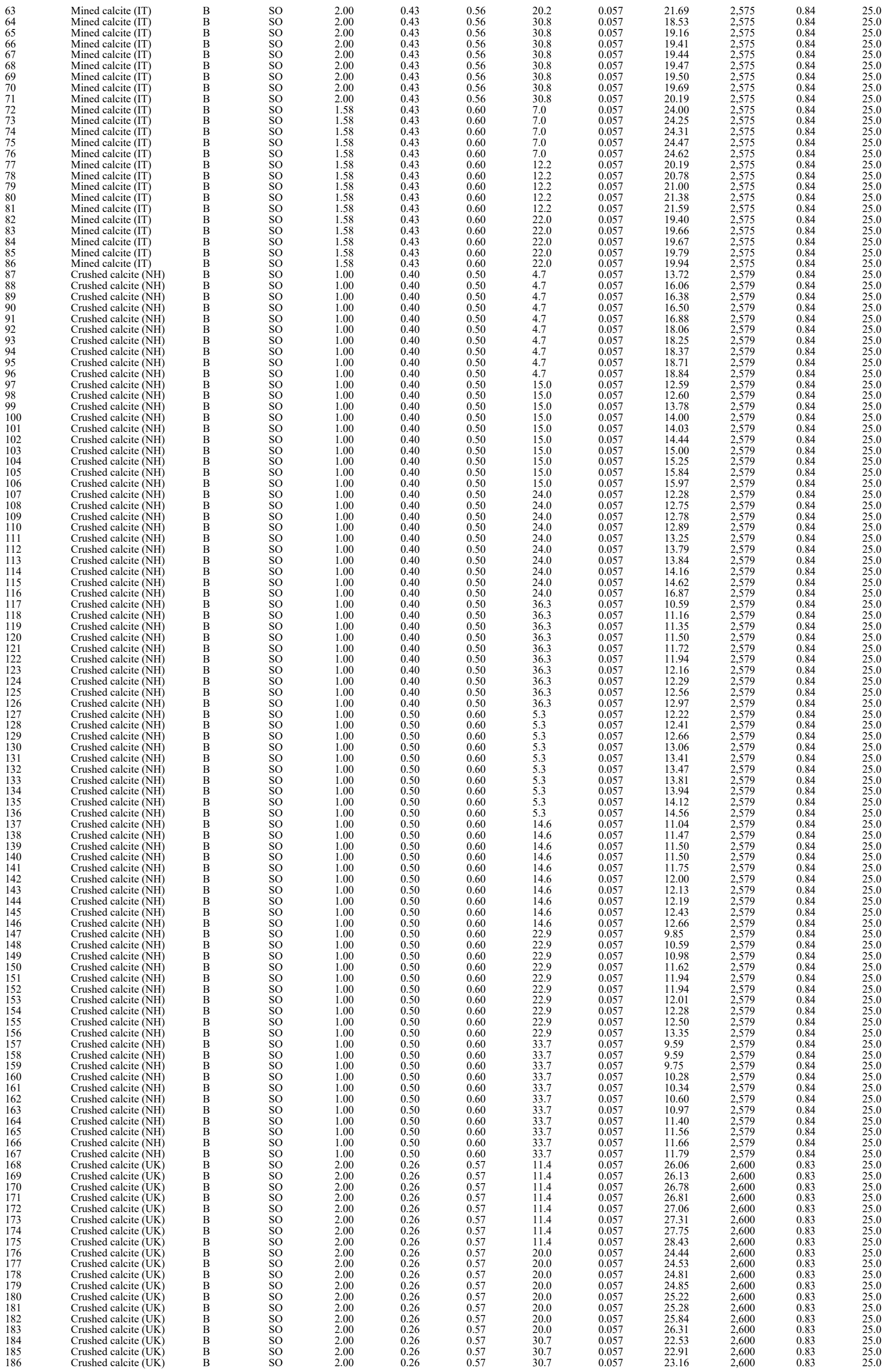




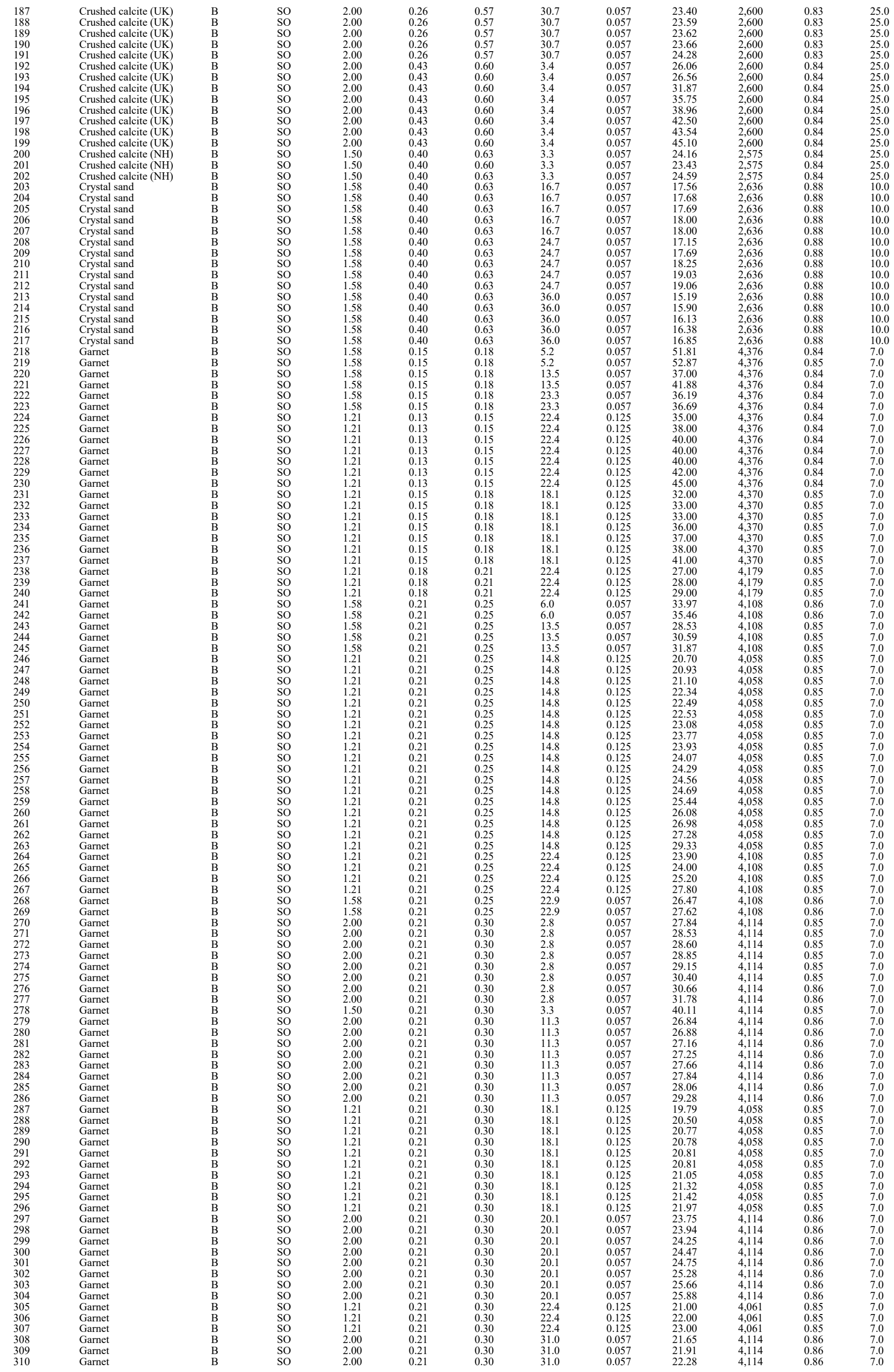




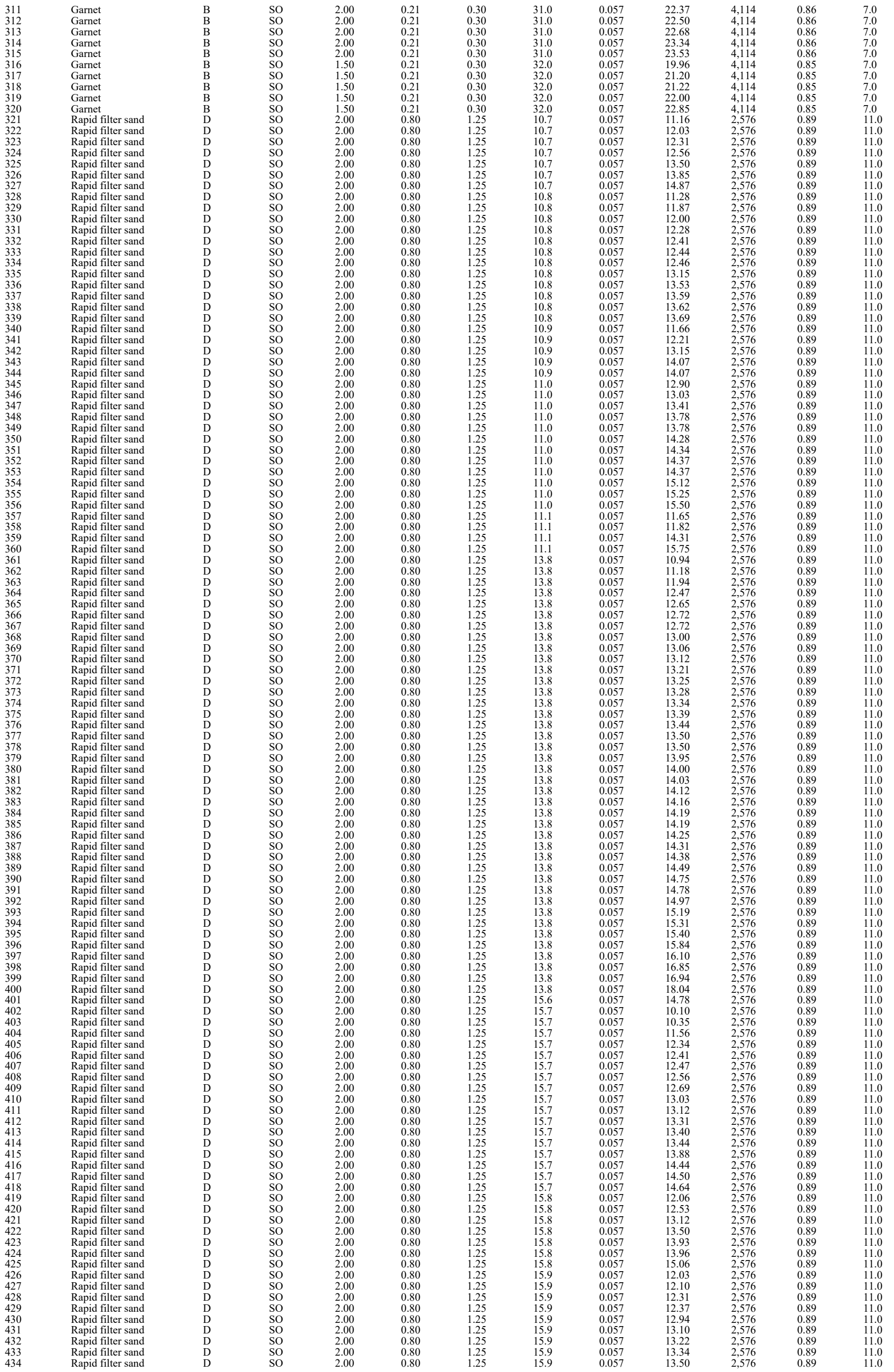




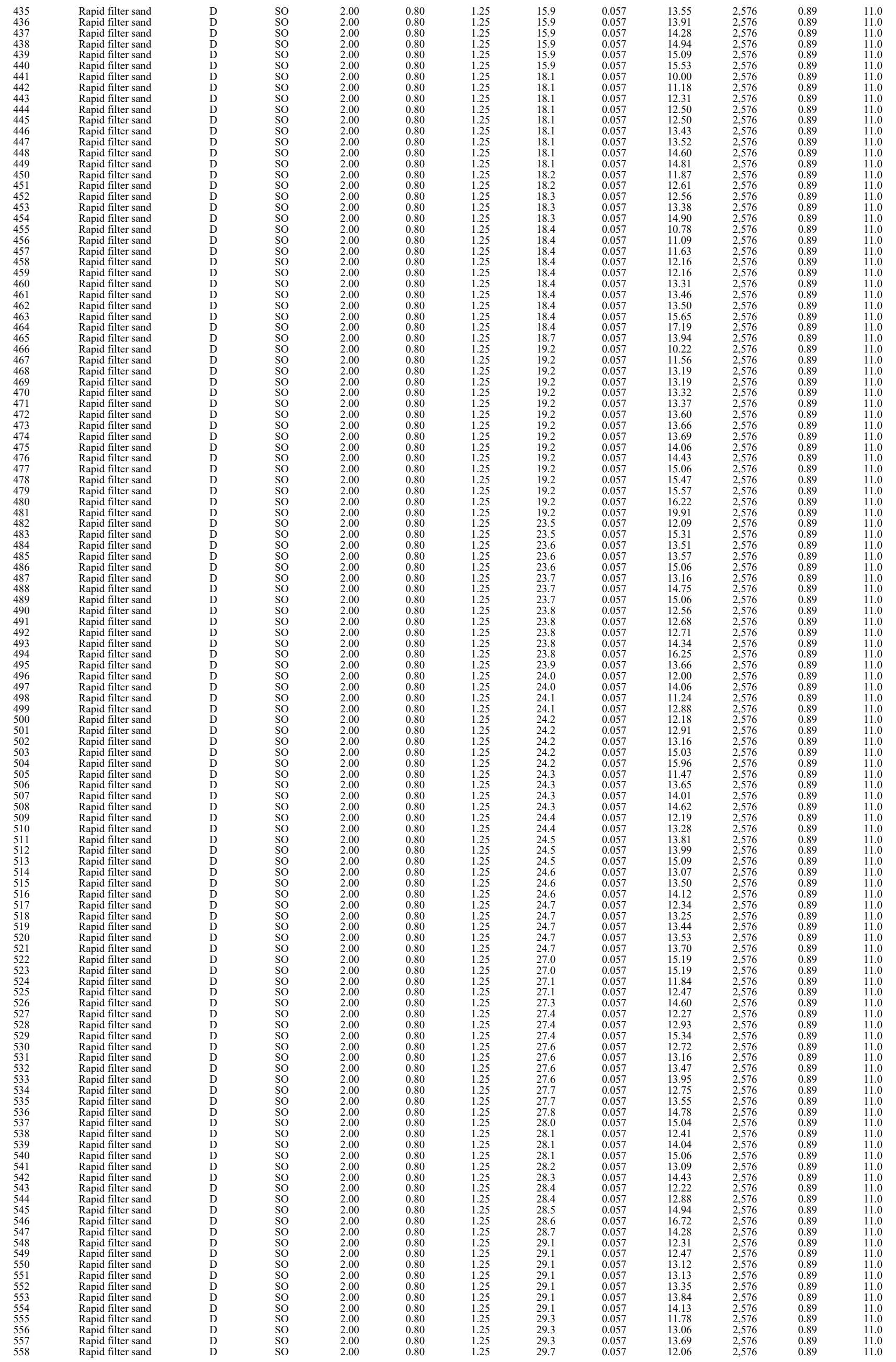




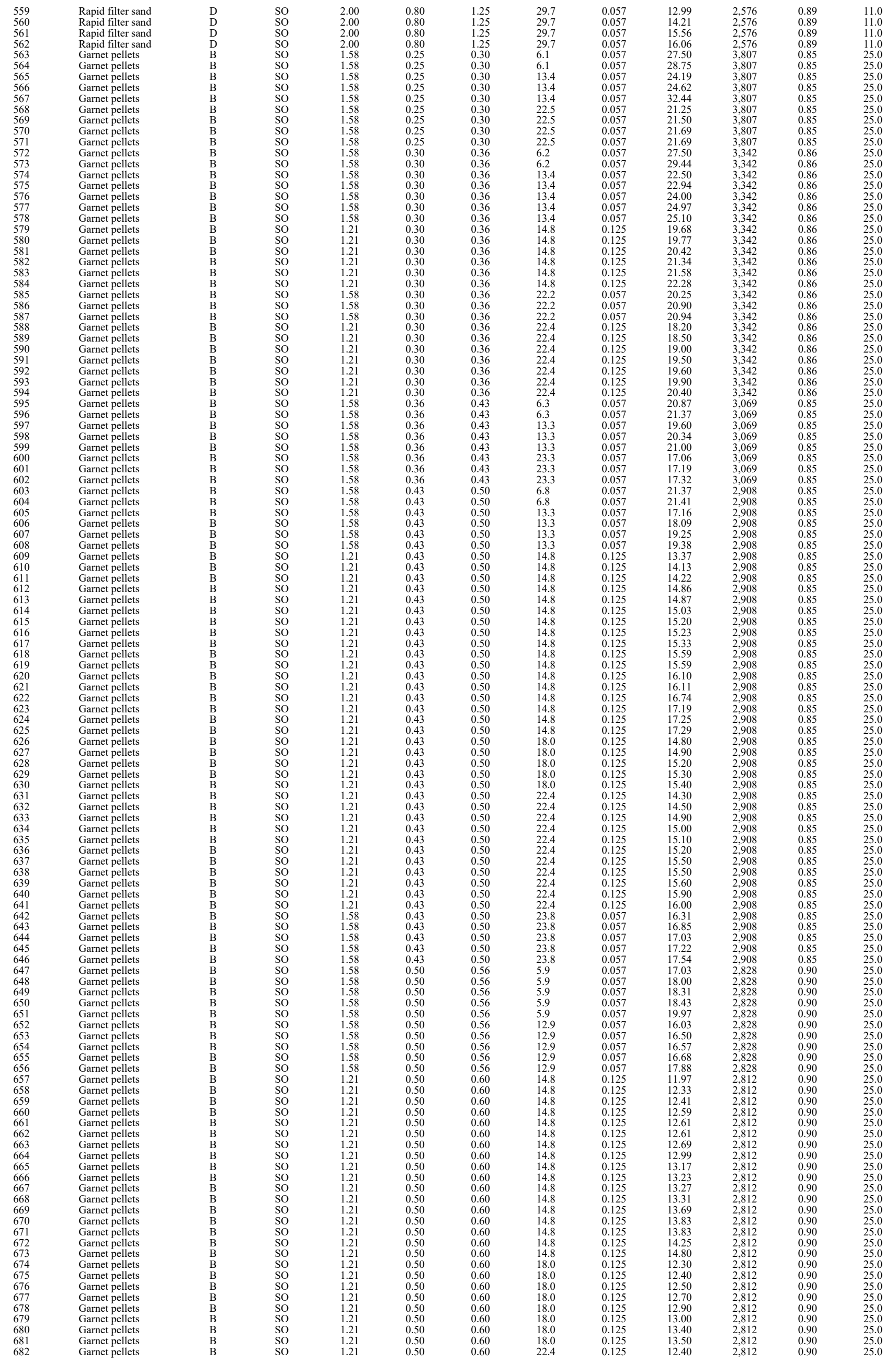




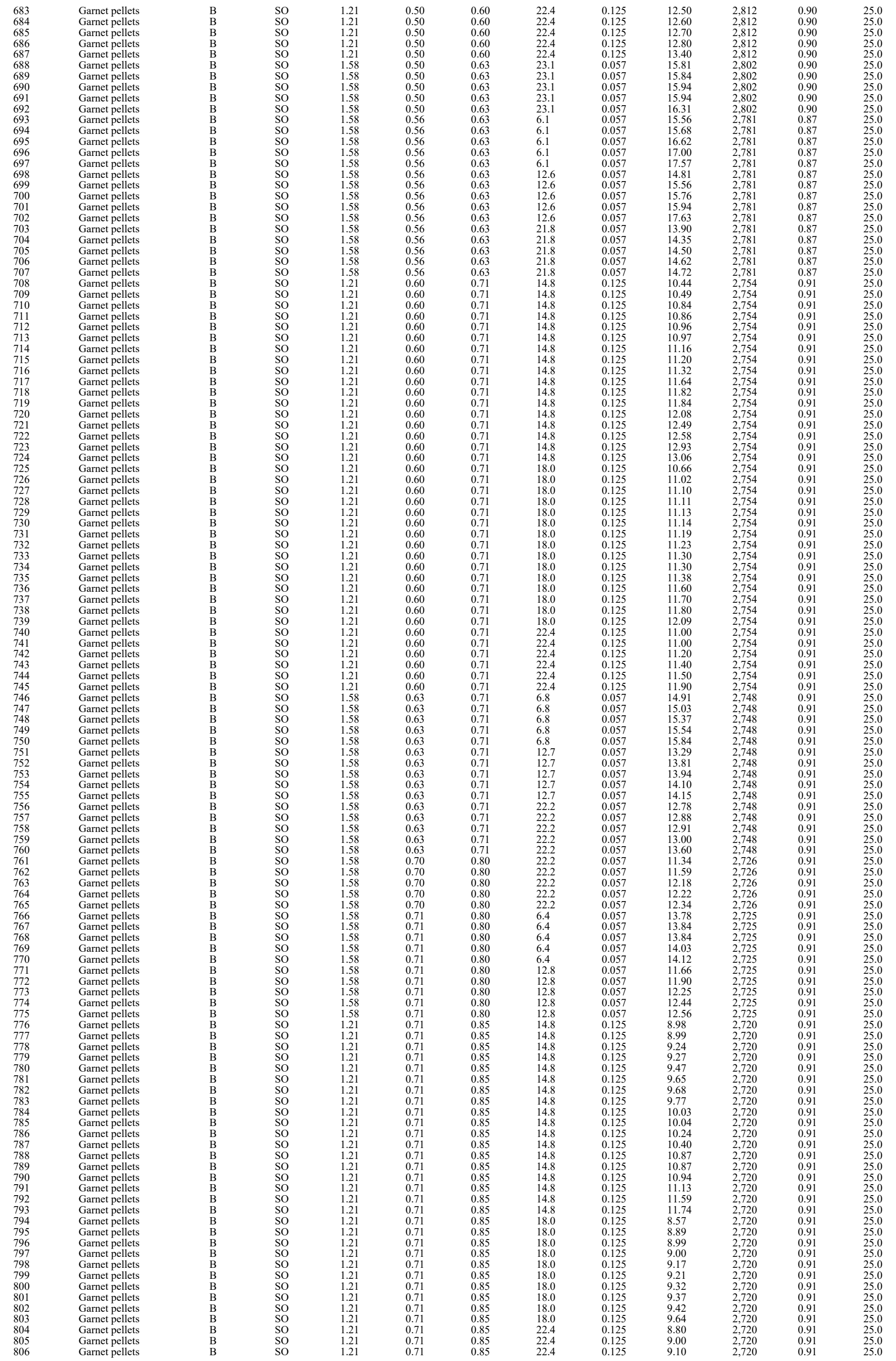




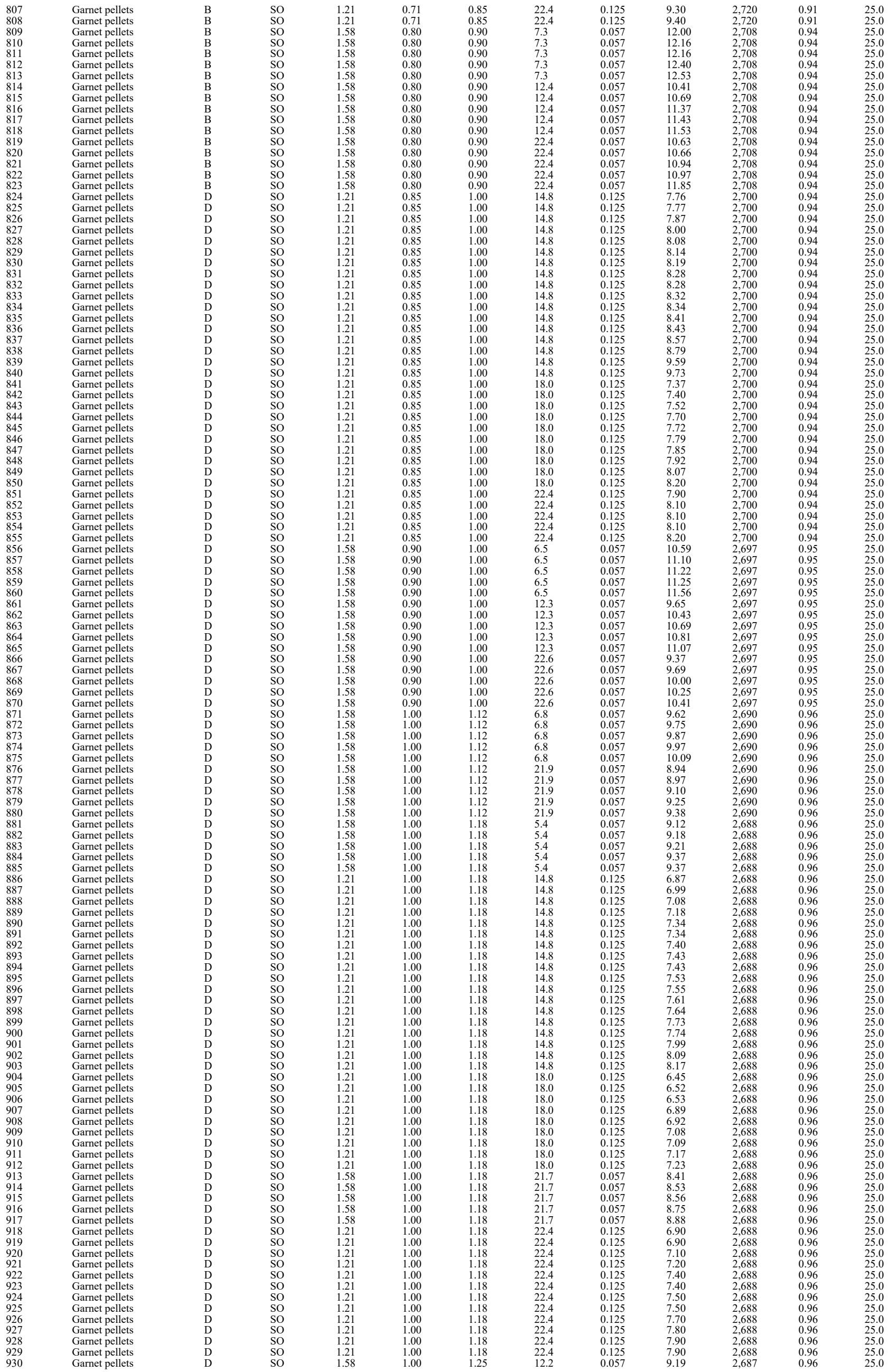




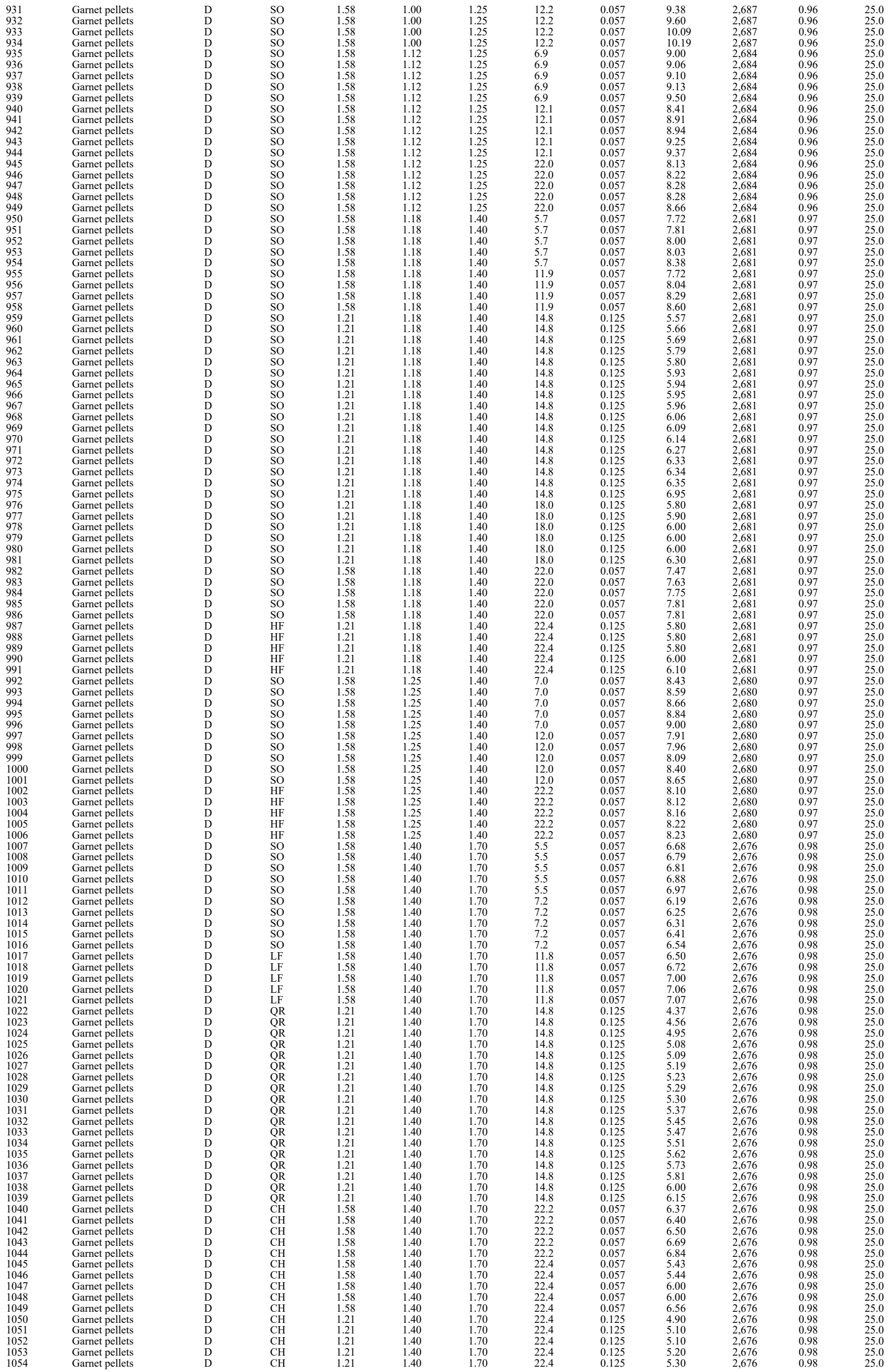




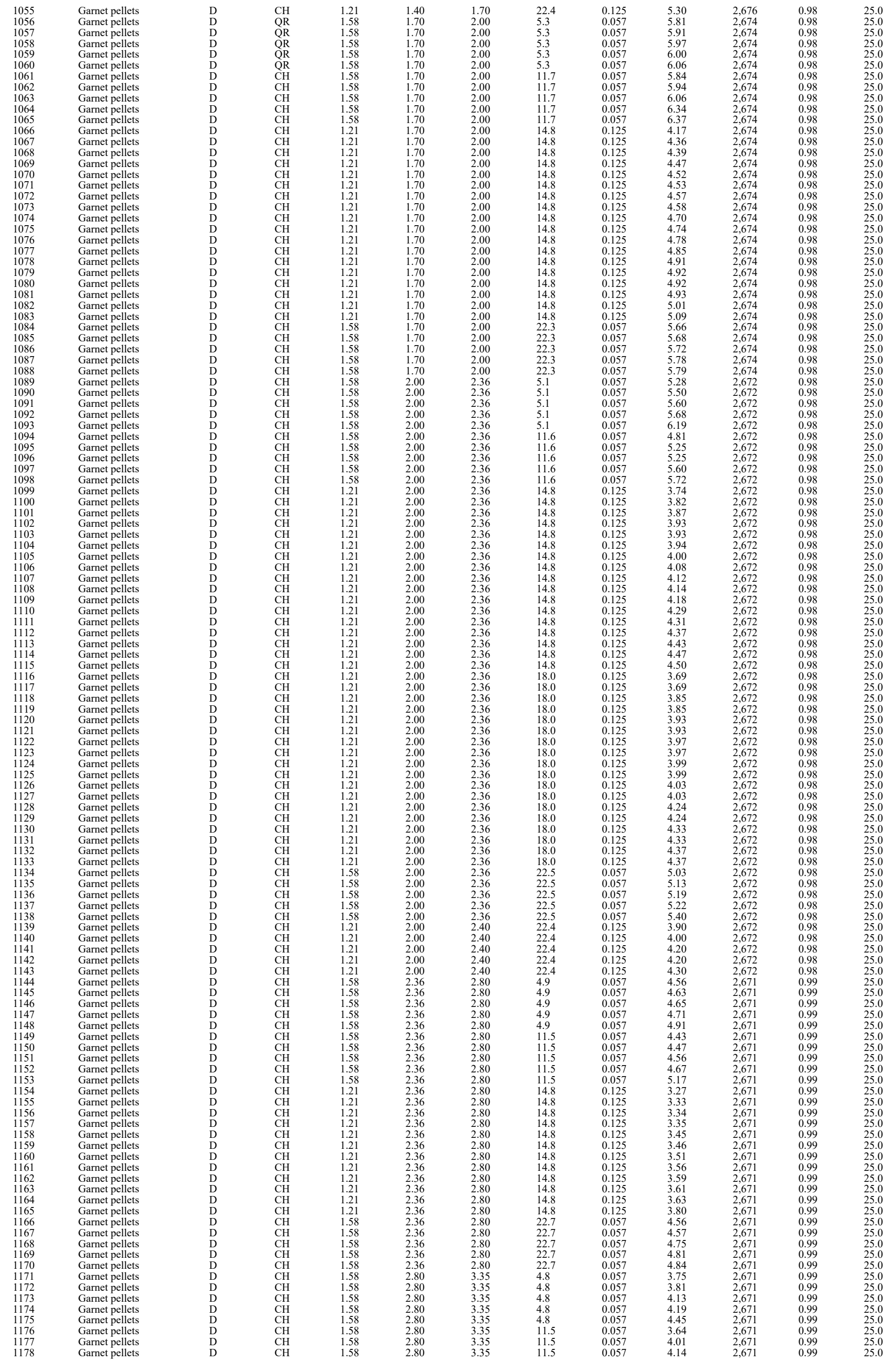




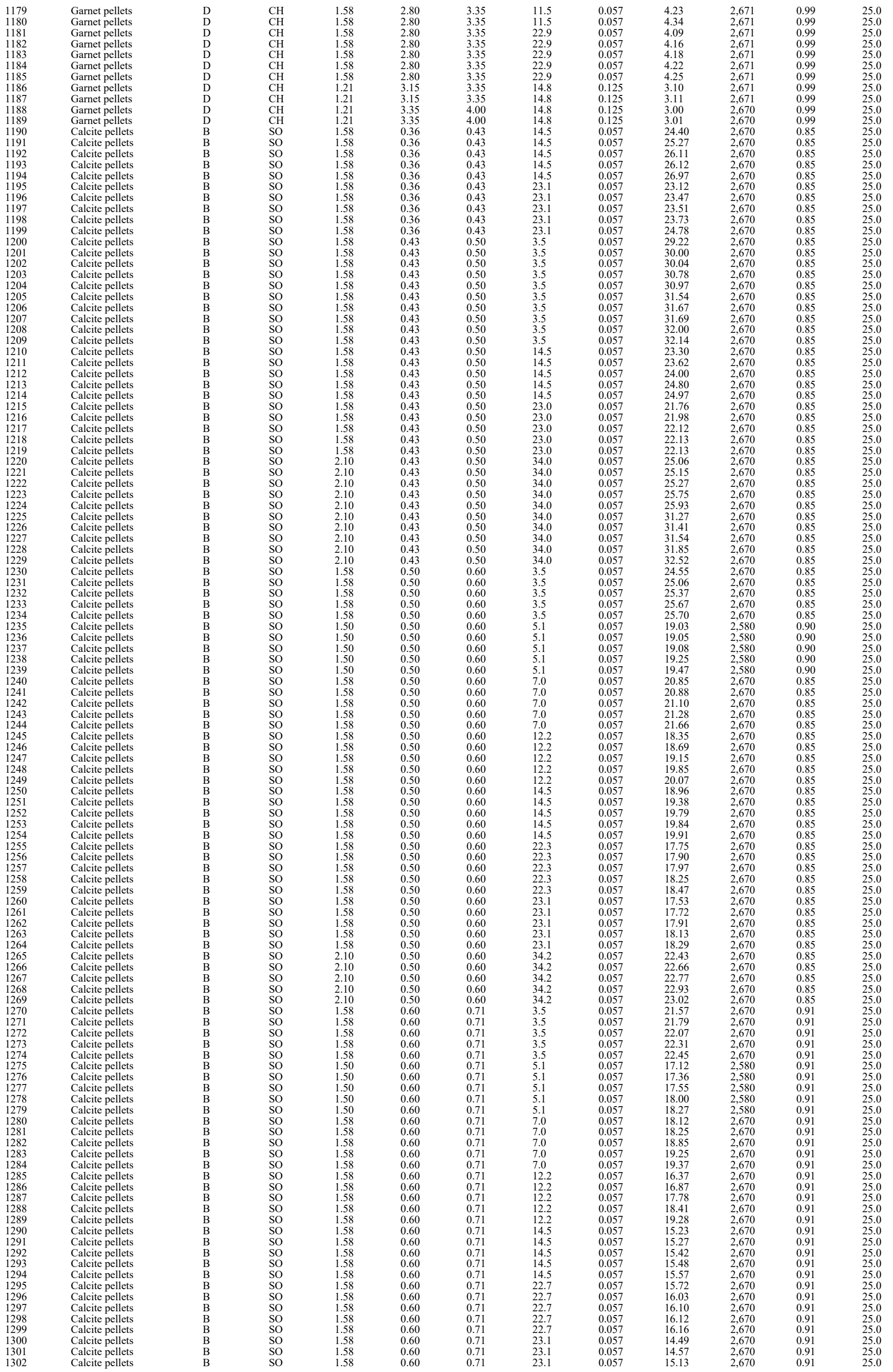




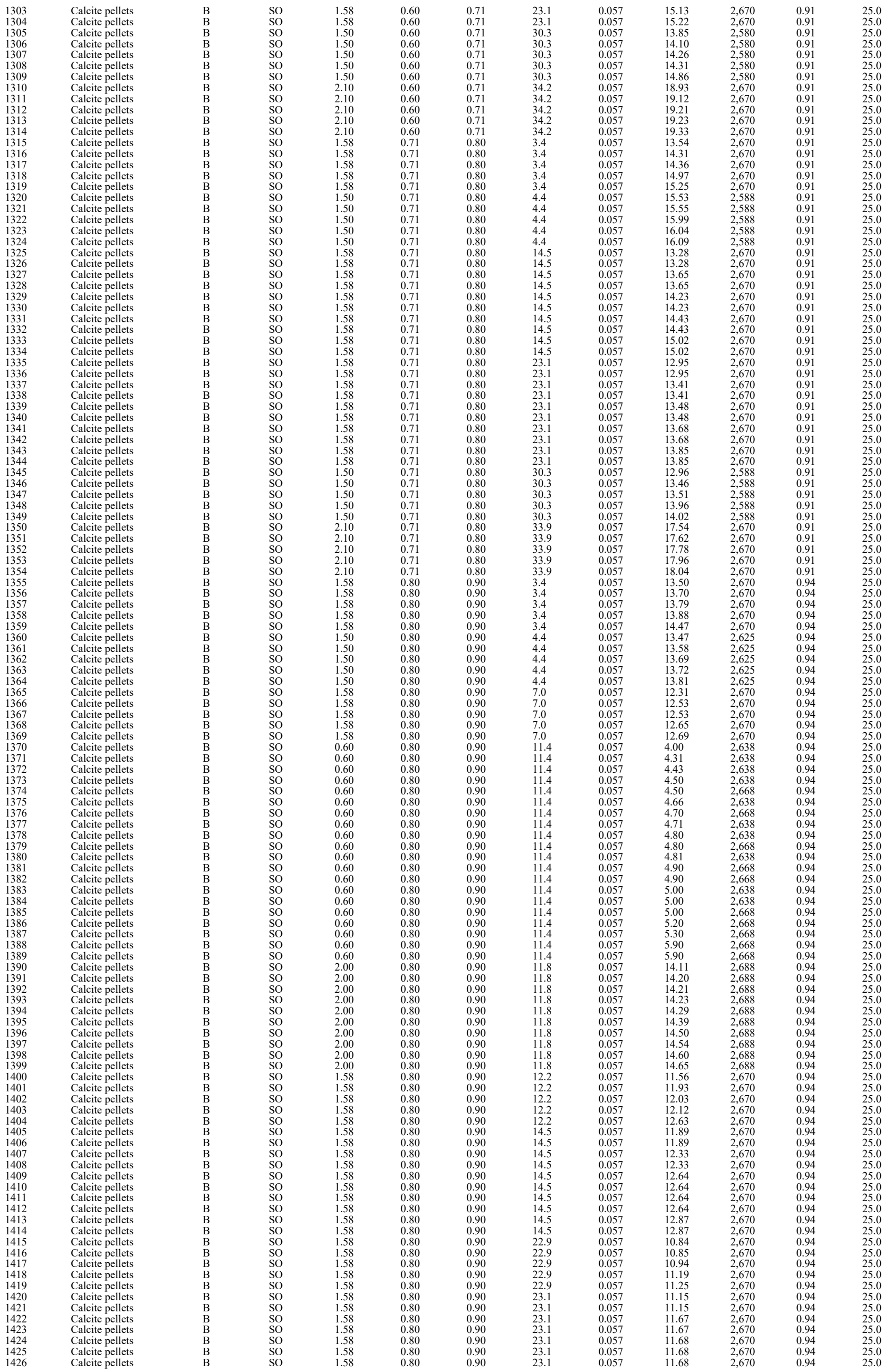




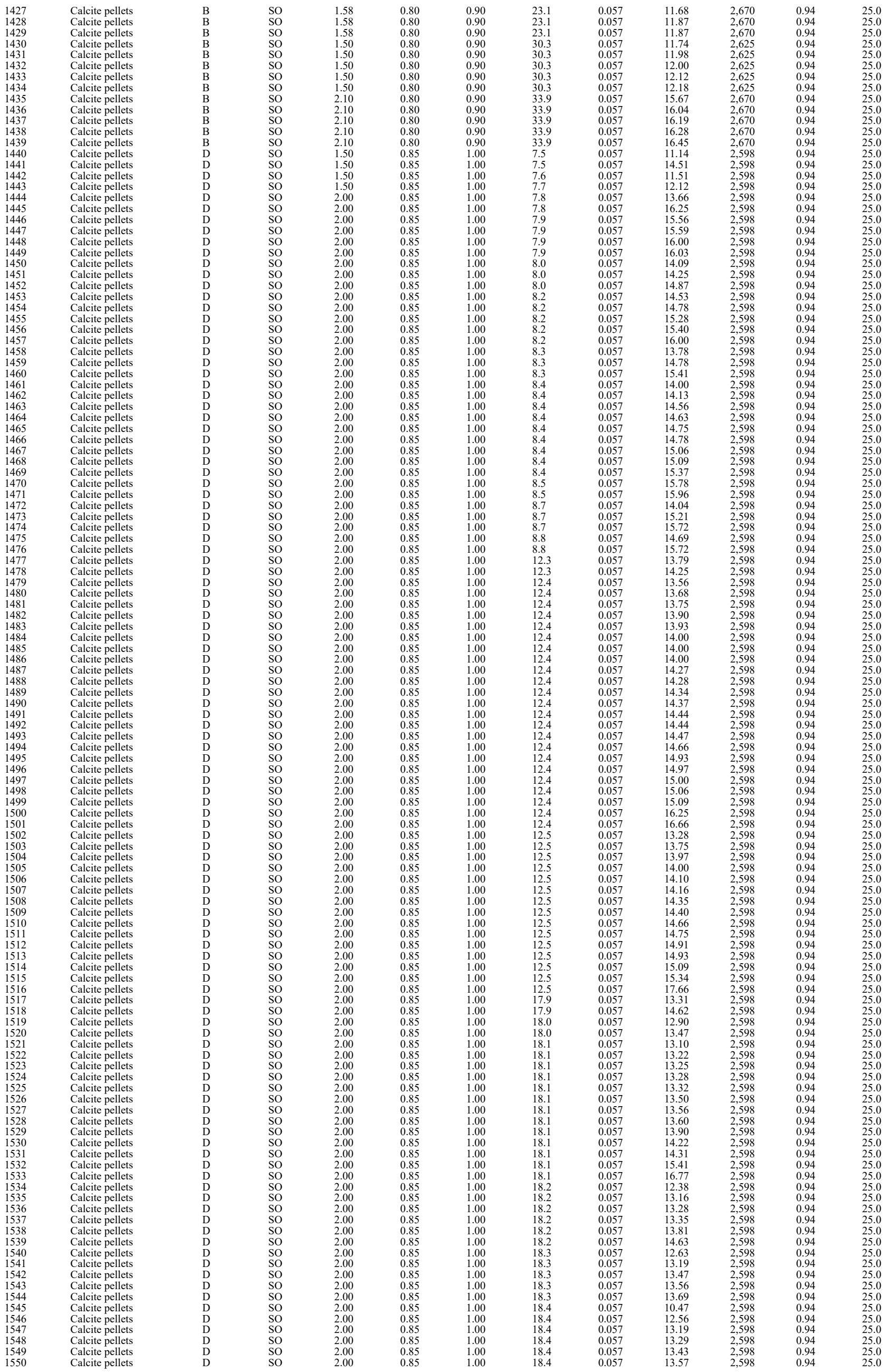




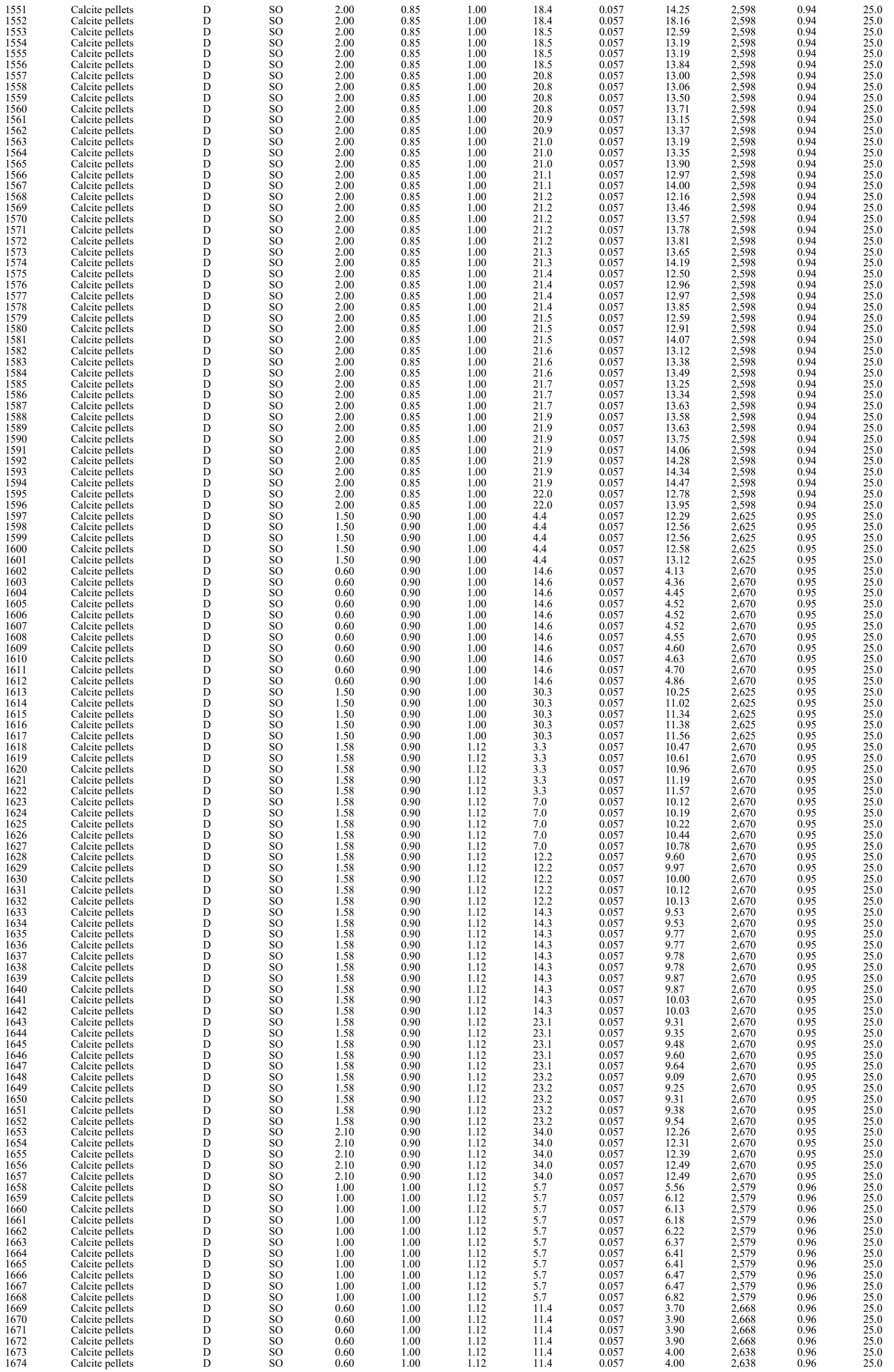




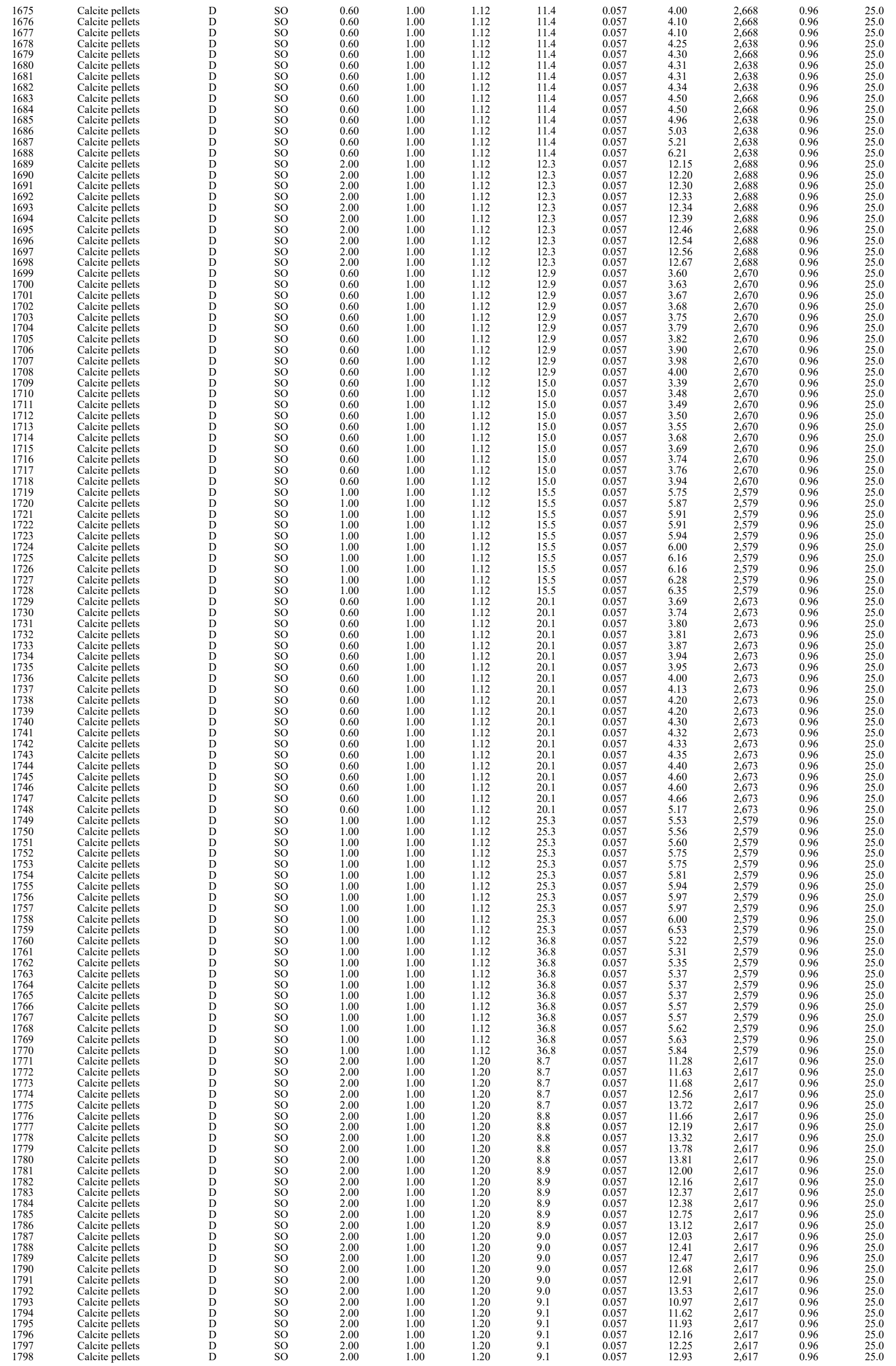




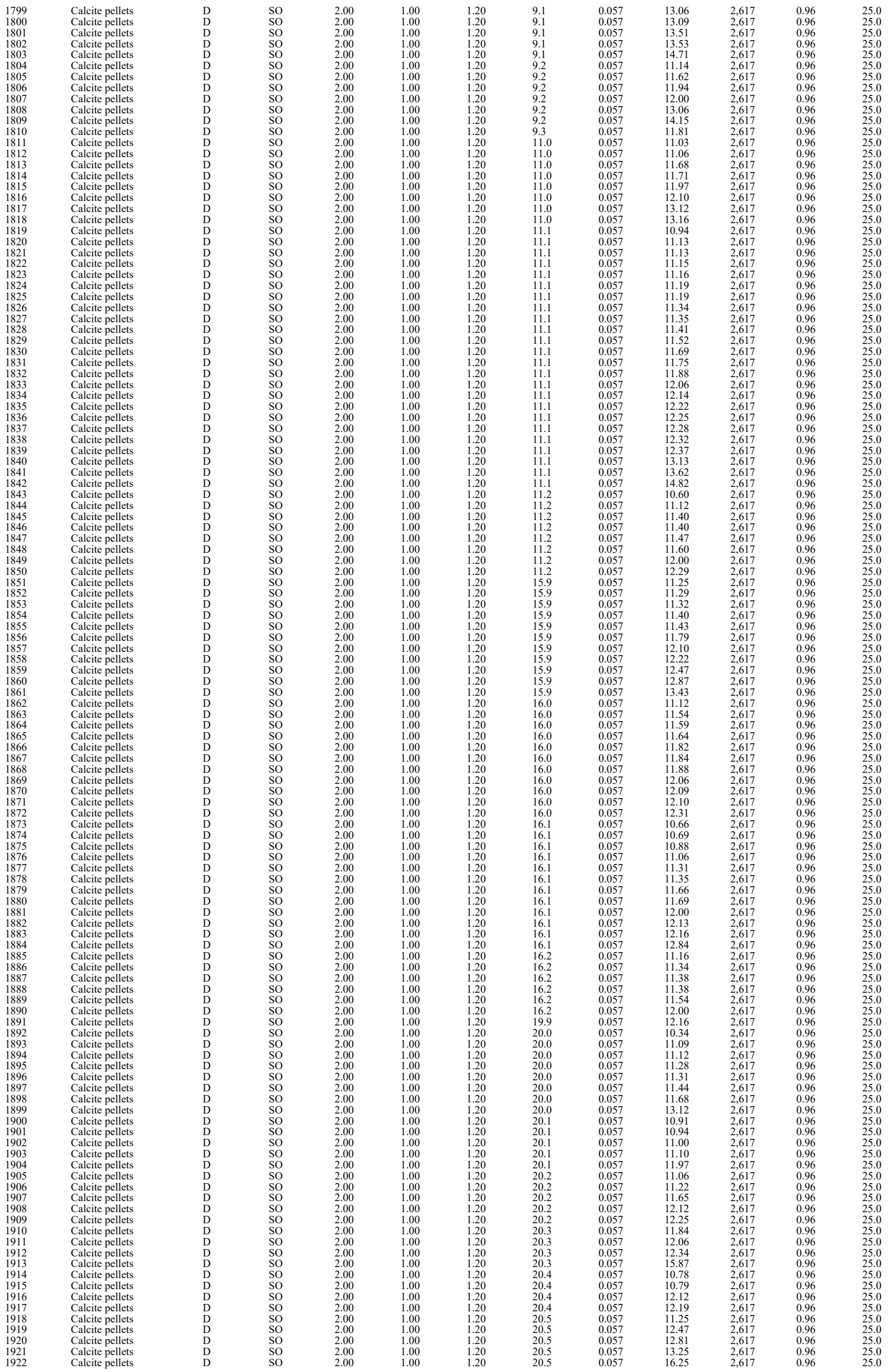




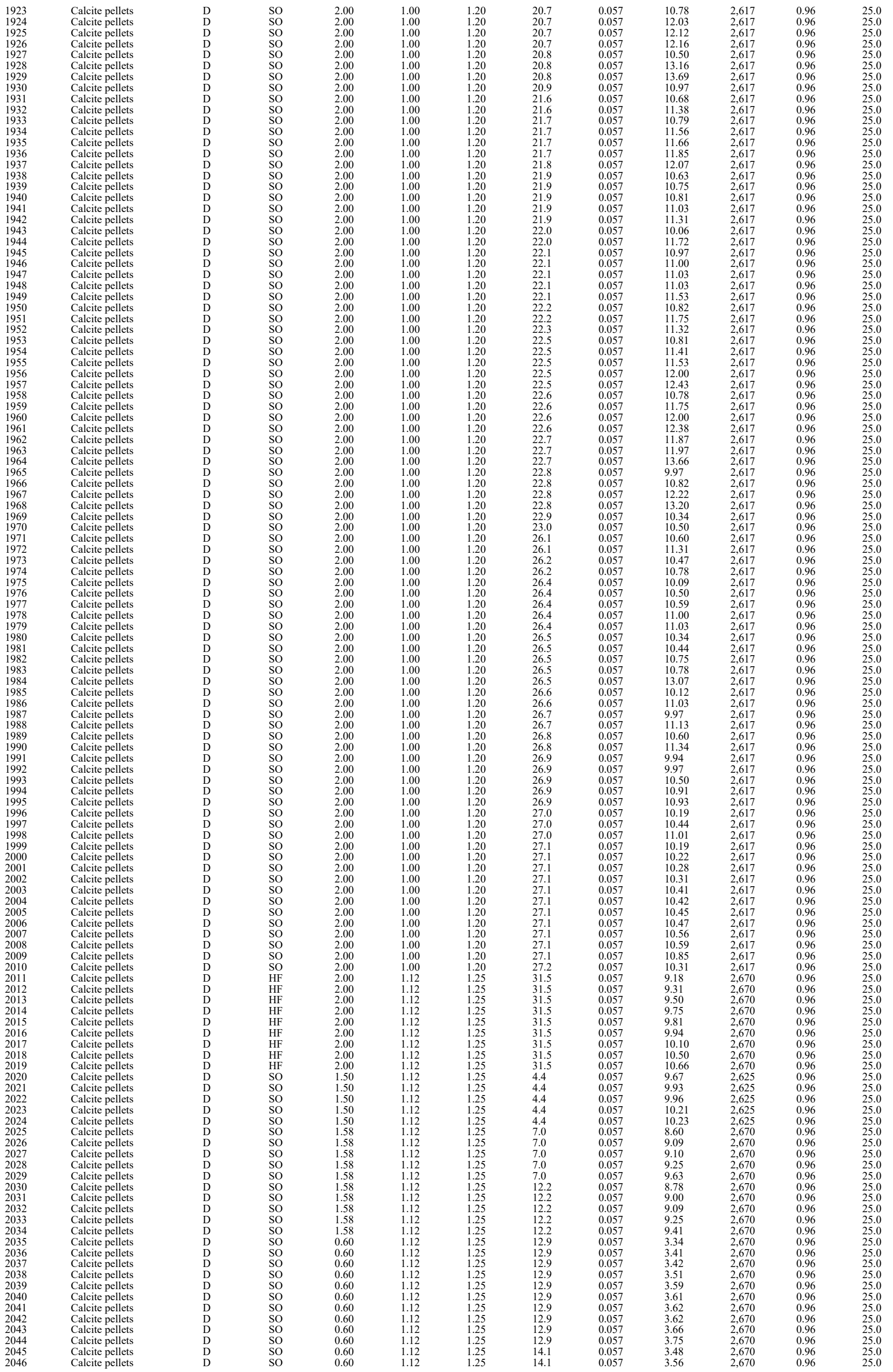




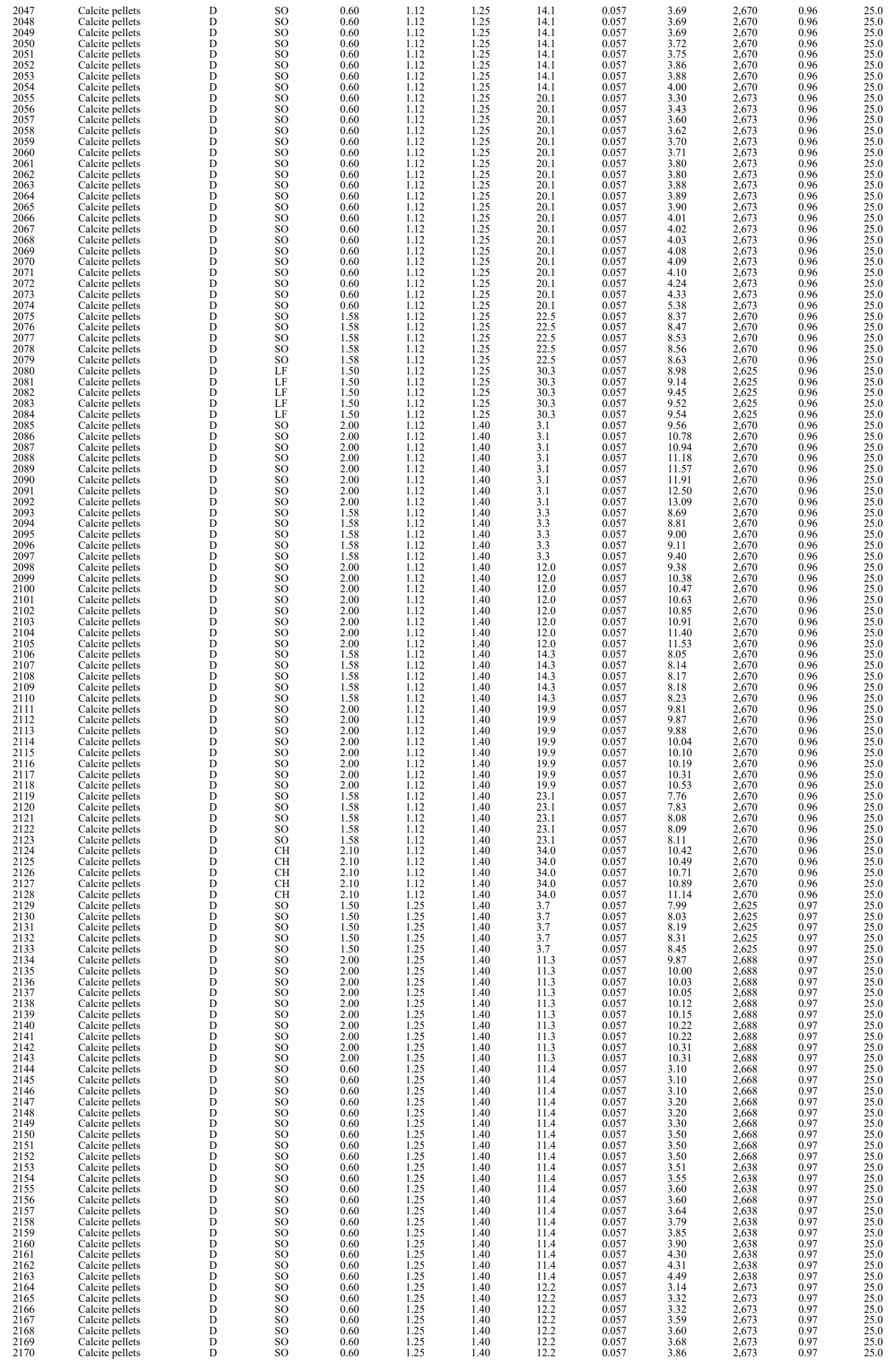




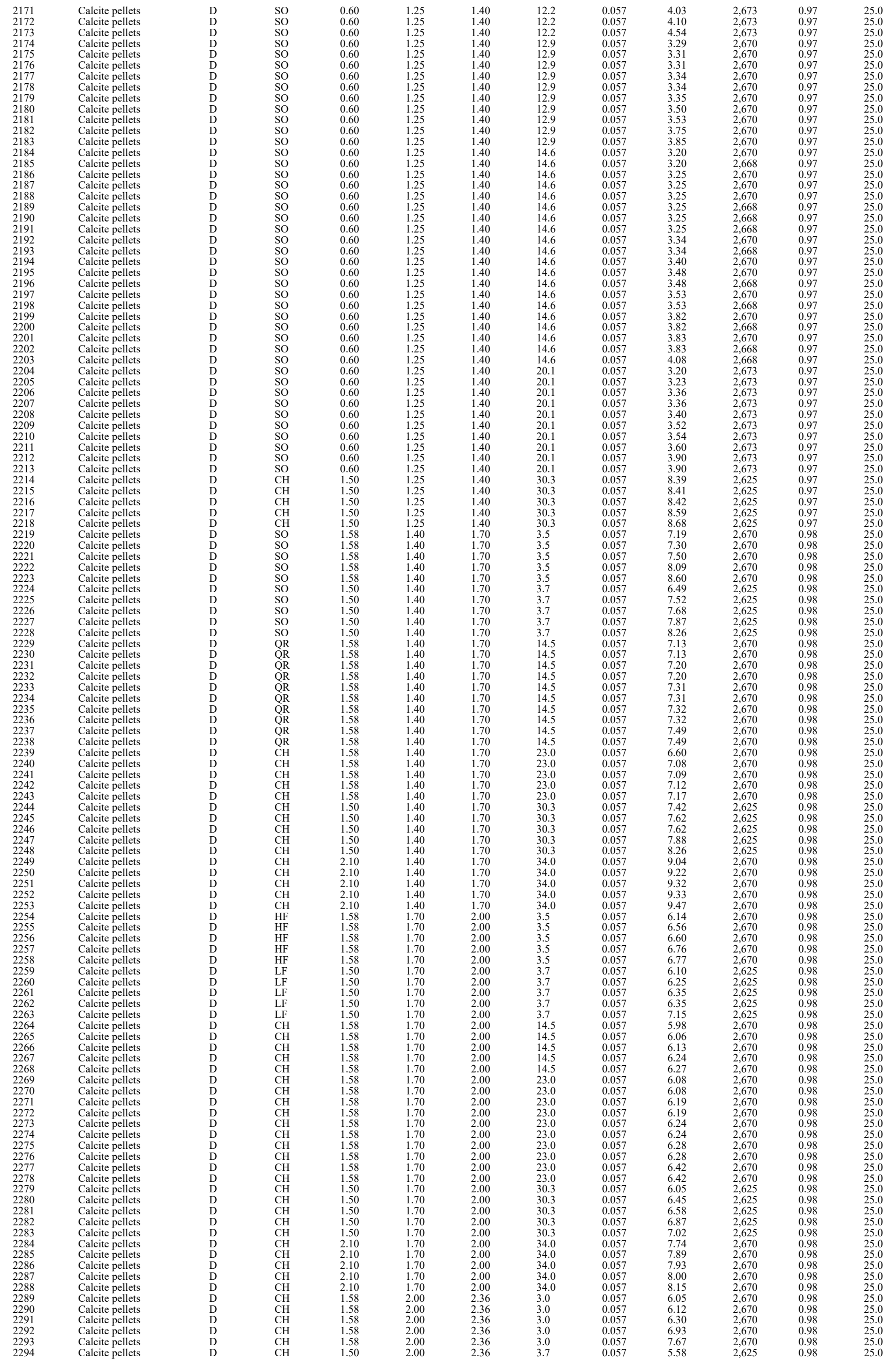




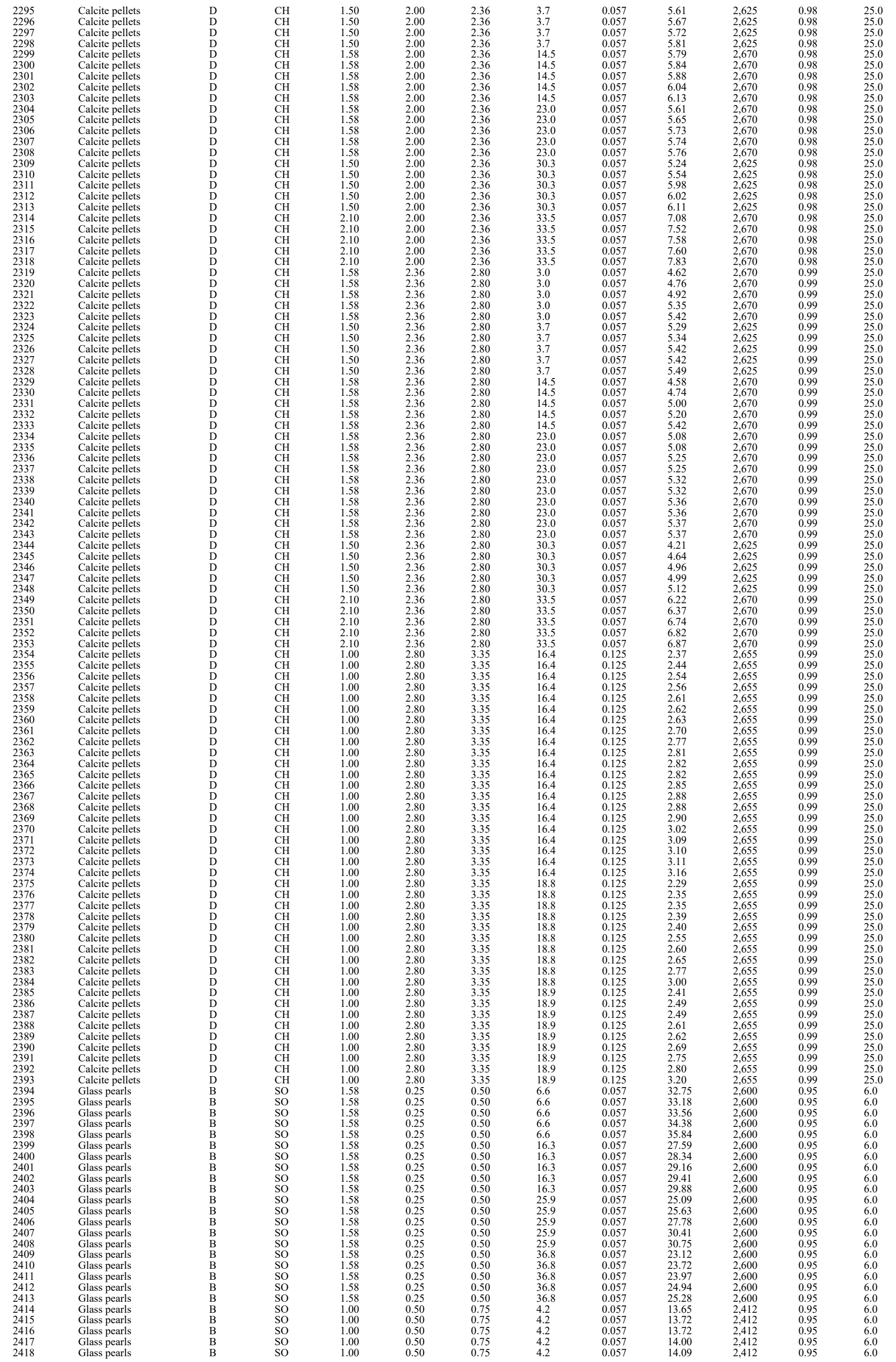




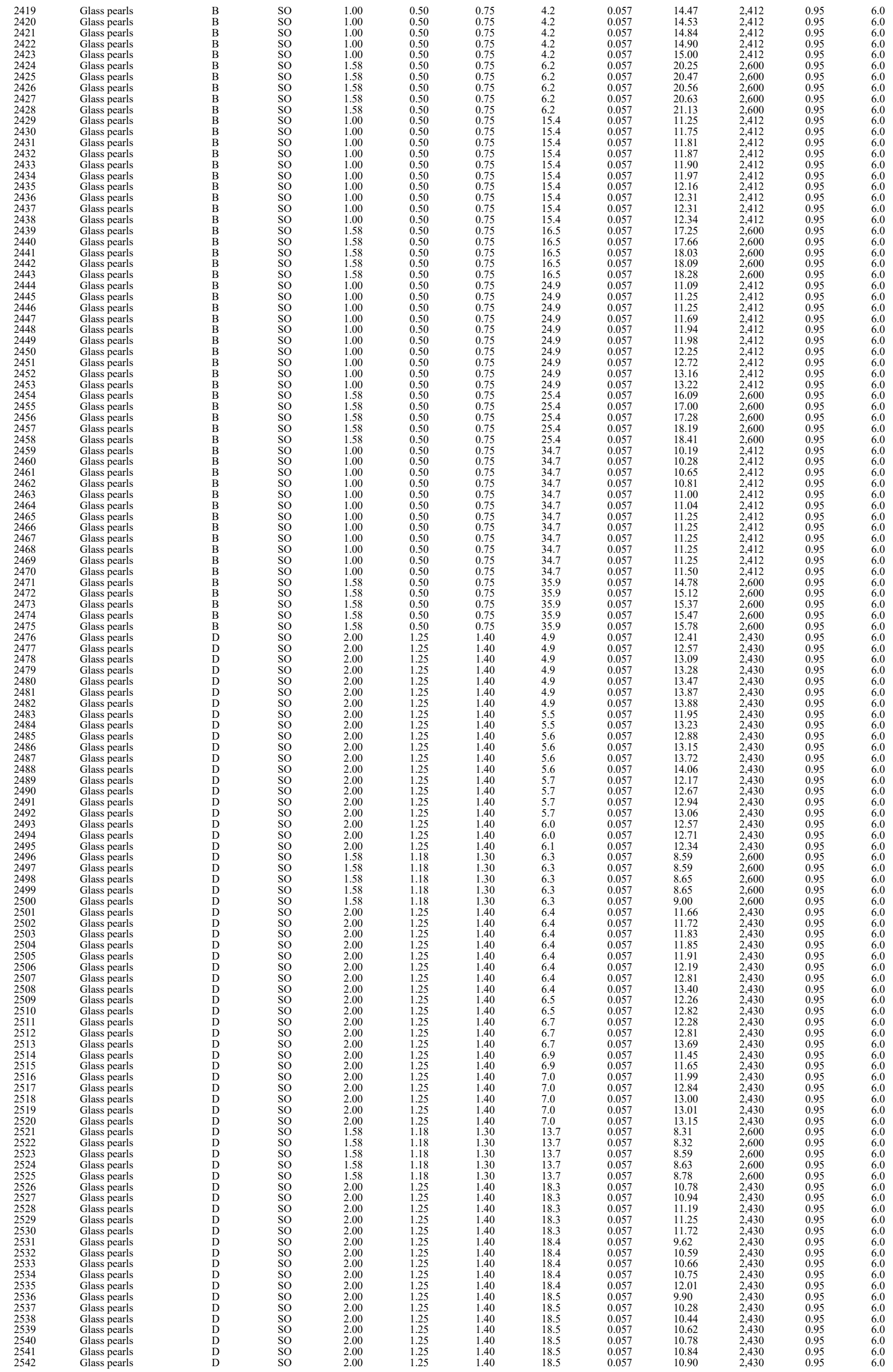




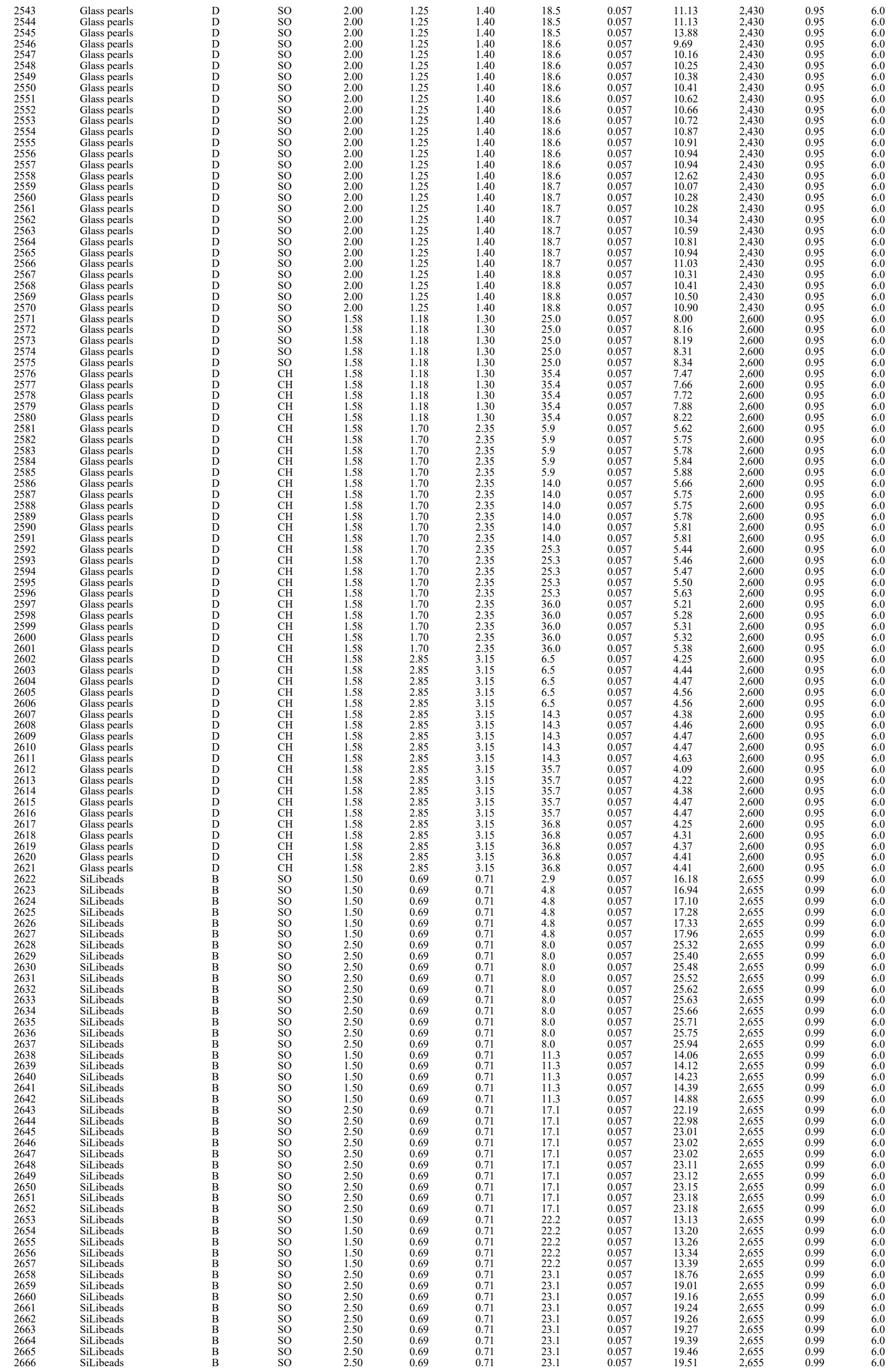




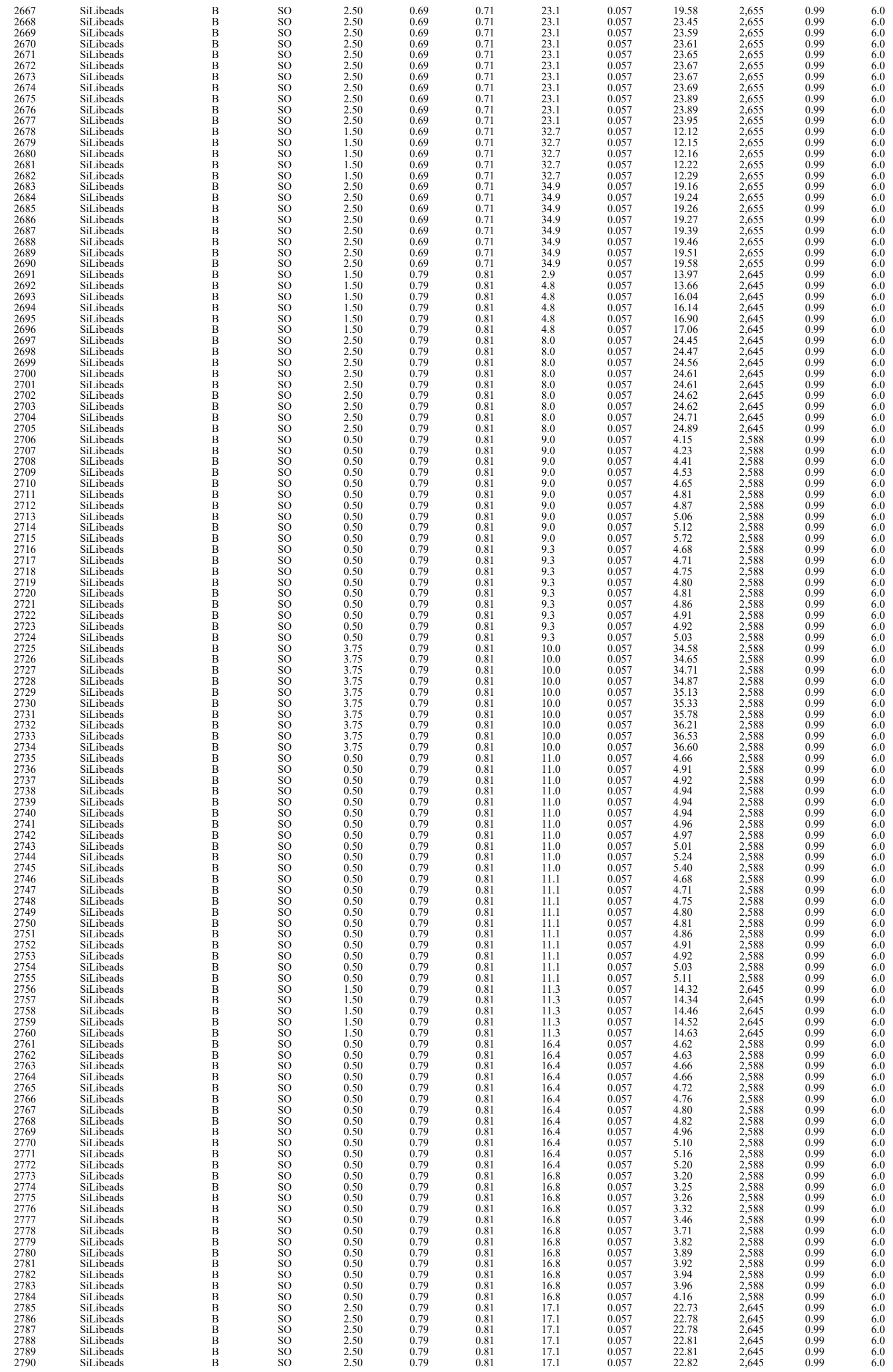




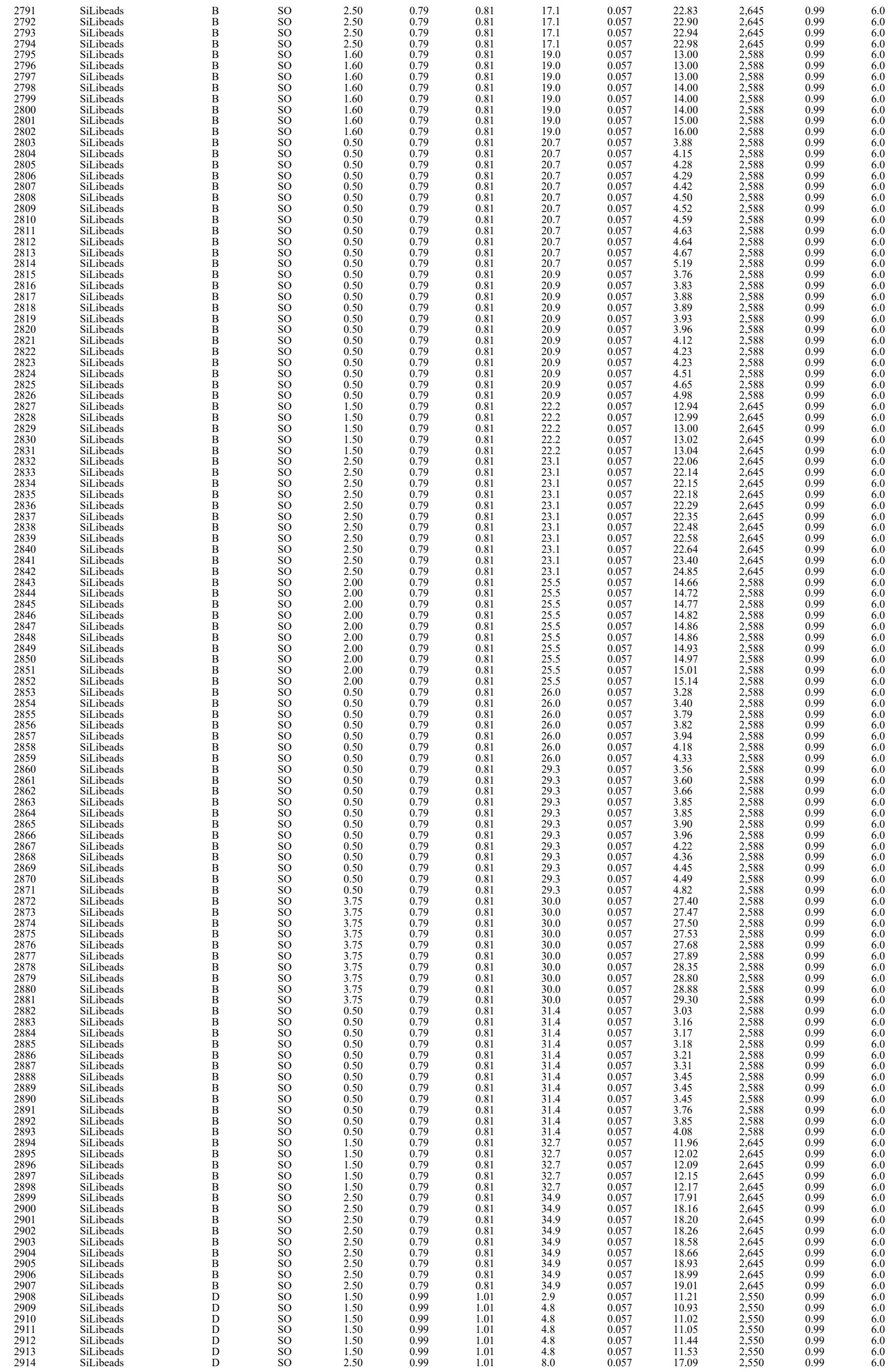




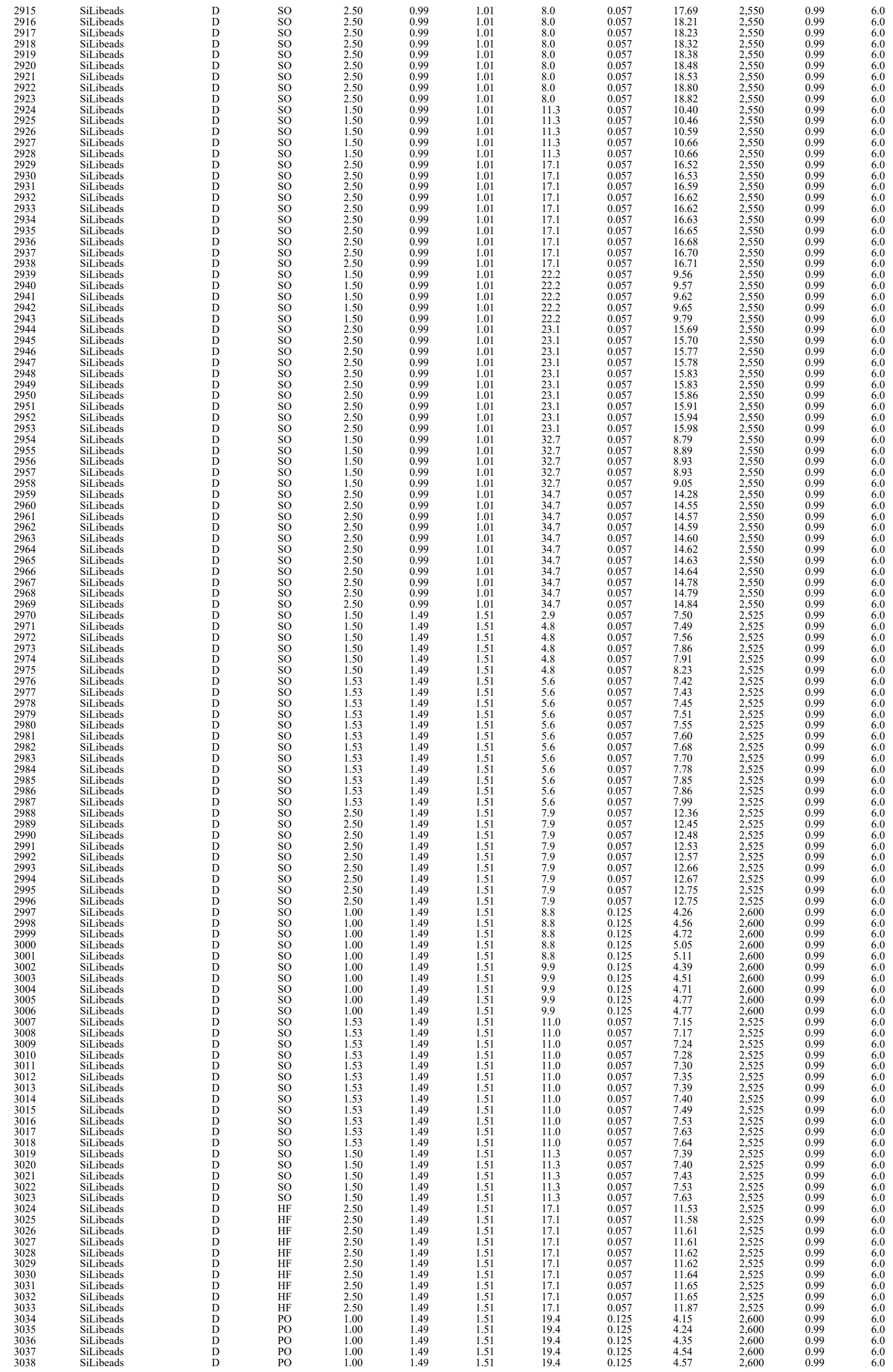




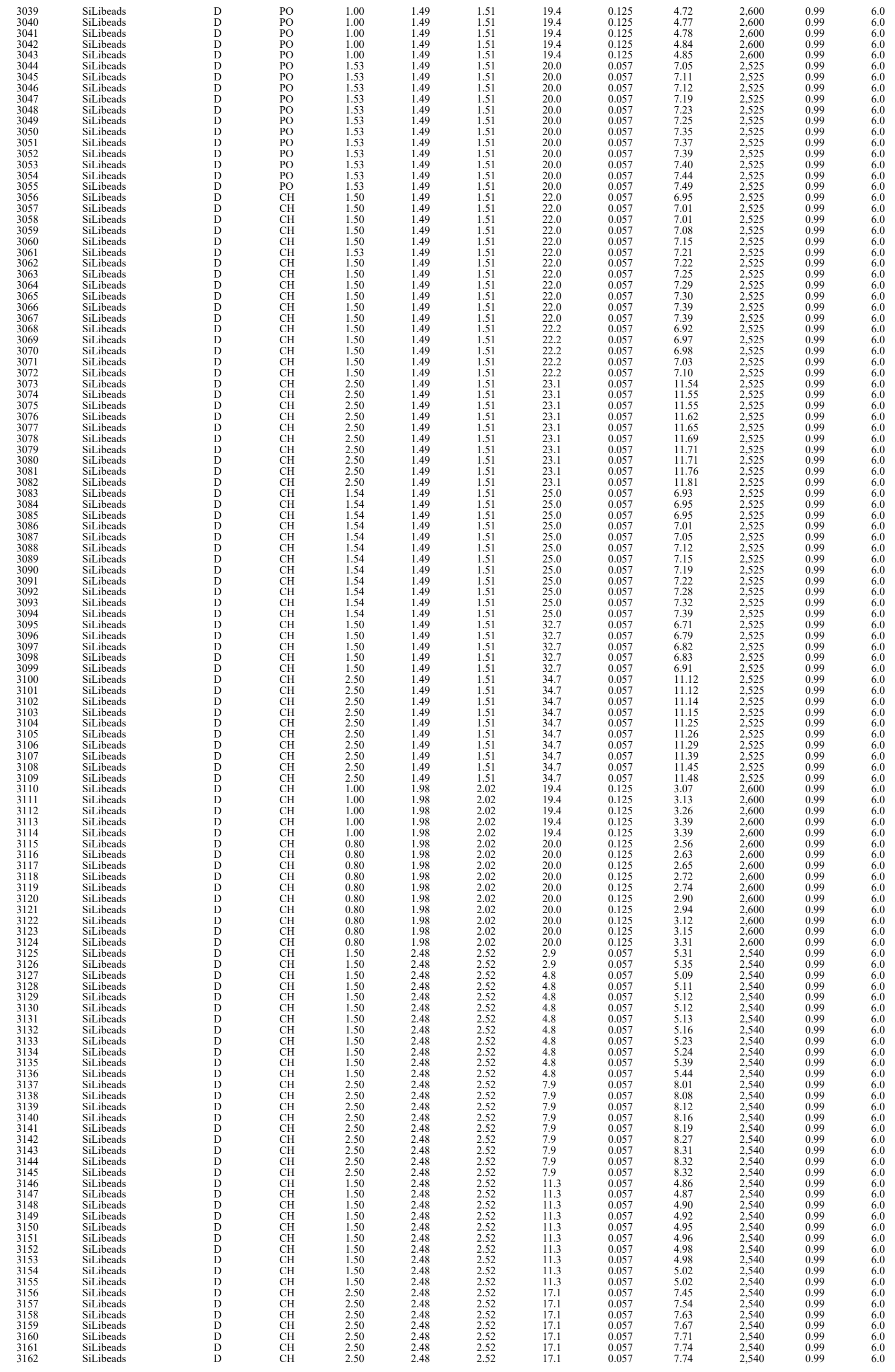




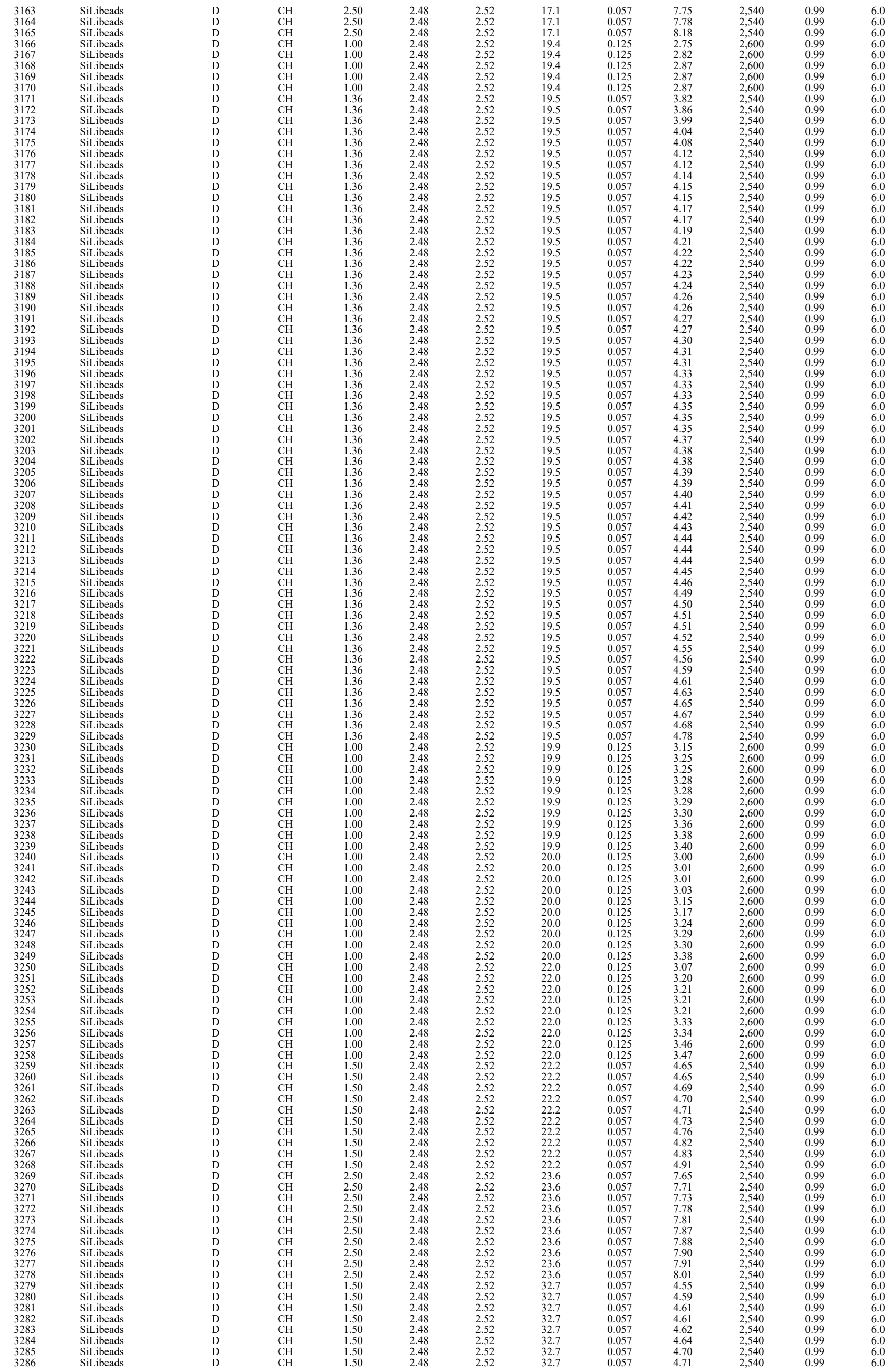




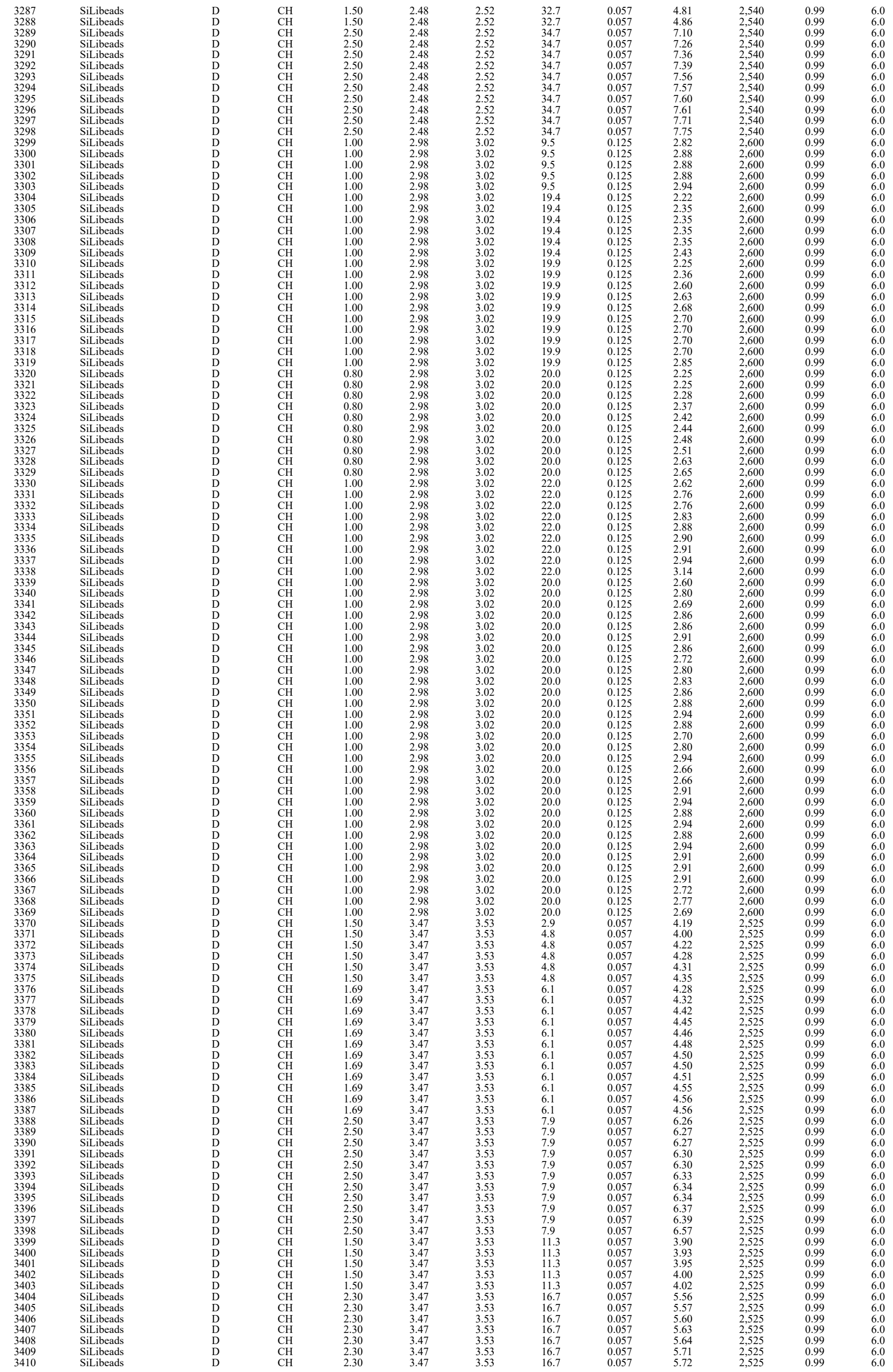




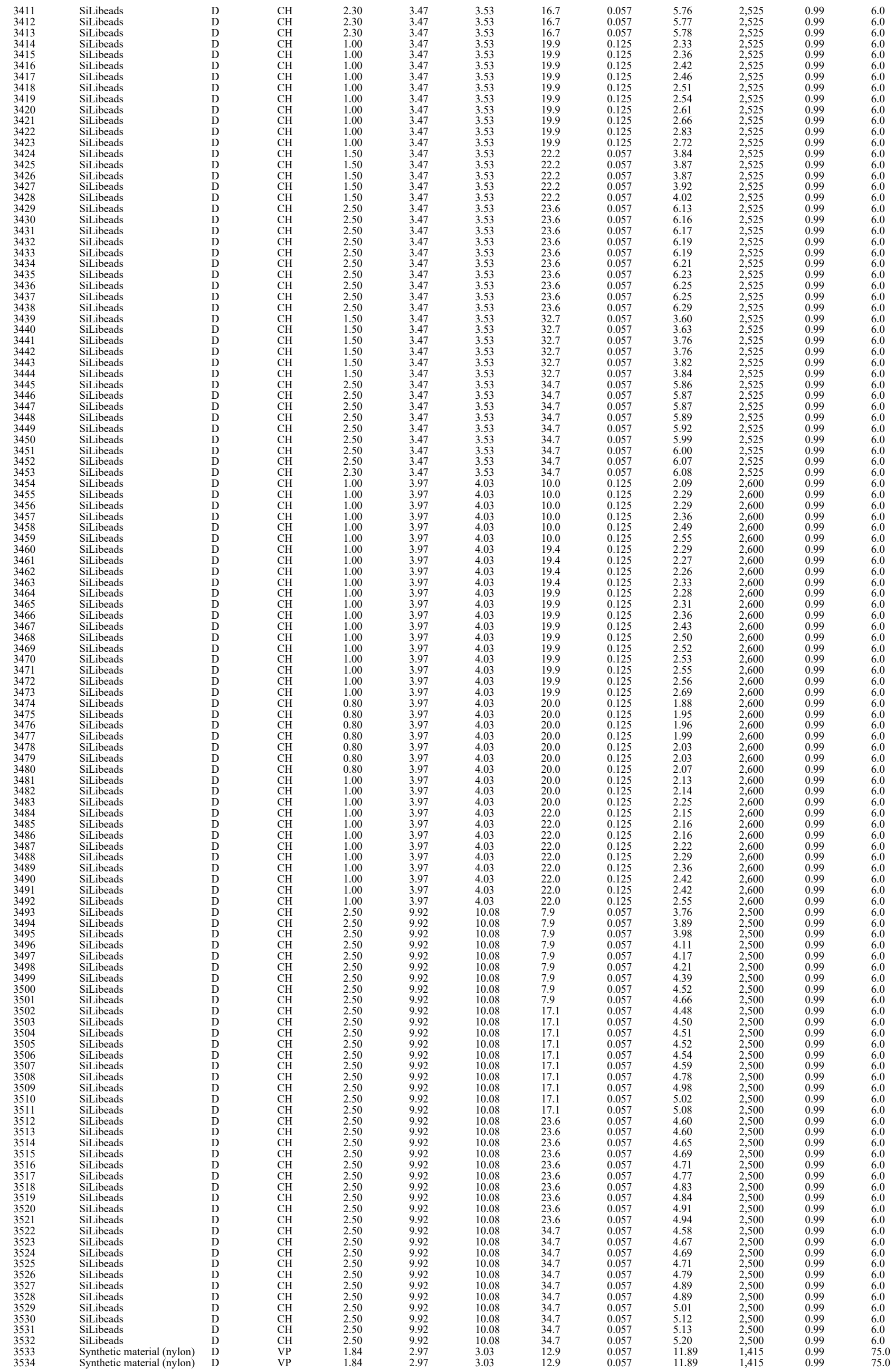




\begin{tabular}{|c|c|c|c|}
\hline 3535 & Synthetic material (nylon) & $\mathrm{D}$ & VP \\
\hline 3536 & Synthetic material (nylon) & D & VP \\
\hline 3537 & Synthetic material (nylon) & D & VP \\
\hline 3538 & Synthetic material (nylon) & $\mathrm{D}$ & VP \\
\hline 3539 & Synthetic material (nylon) & $\mathrm{D}$ & VP \\
\hline 3540 & Synthetic material (nylon) & $\mathrm{D}$ & VP \\
\hline 3541 & Synthetic material (nylon) & $\mathrm{D}$ & VP \\
\hline 3542 & Synthetic material (nylon) & $\mathrm{D}$ & VP \\
\hline 3543 & Synthetic material (nylon) & $\mathrm{D}$ & VP \\
\hline 3544 & Synthetic material (nylon) & $\mathrm{D}$ & VP \\
\hline 3545 & Synthetic material (nylon) & $\mathrm{D}$ & VP \\
\hline 3546 & Synthetic material (nylon) & D & $\begin{array}{l}\mathrm{VP} \\
\mathrm{VP}\end{array}$ \\
\hline 3547 & Synthetic material (nylon) & $\mathrm{D}$ & VP \\
\hline 3548 & Synthetic material (nylon) & $\mathrm{D}$ & VP \\
\hline 3549 & Synthetic material (nylon) & $\mathrm{D}$ & VP \\
\hline 3550 & Synthetic material (nylon) & $\mathrm{D}$ & VP \\
\hline 3551 & Synthetic material (nylon) & $\mathrm{D}$ & VP \\
\hline 3552 & Synthetic material (nylon) & $\mathrm{D}$ & VP \\
\hline 3553 & Synthetic material (nylon) & $\mathrm{D}$ & VP \\
\hline 3554 & Synthetic material (nylon) & $\mathrm{D}$ & VP \\
\hline 3555 & Synthetic material (nylon) & $\mathrm{D}$ & VP \\
\hline 3556 & Synthetic material (nylon) & $\mathrm{D}$ & VP \\
\hline 3557 & Synthetic material (nylon) & D & VP \\
\hline 3558 & Synthetic material (nylon) & $\mathrm{D}$ & $\mathrm{CH}$ \\
\hline 3559 & Synthetic material (nylon) & $\mathrm{D}$ & $\mathrm{CH}$ \\
\hline 3560 & Synthetic material (nylon) & $\mathrm{D}$ & $\mathrm{CH}$ \\
\hline 3561 & Synthetic material (nylon) & D & $\mathrm{CH}$ \\
\hline 3562 & Synthetic material (nylon) & $\mathrm{D}$ & $\mathrm{CH}$ \\
\hline 3563 & Synthetic material (nylon) & $\mathrm{D}$ & $\mathrm{CH}$ \\
\hline 3564 & Synthetic material (nylon) & $\mathrm{D}$ & $\mathrm{CH}$ \\
\hline 3565 & Synthetic material (nylon) & D & $\mathrm{CH}$ \\
\hline 3566 & Synthetic material (nylon) & $\mathrm{D}$ & $\mathrm{CH}$ \\
\hline 3567 & Synthetic material (nylon) & D & $\mathrm{CH}$ \\
\hline 3568 & Synthetic material (nylon) & $\mathrm{D}$ & $\mathrm{CH}$ \\
\hline 3569 & Synthetic material (nylon) & $\mathrm{D}$ & $\mathrm{CH}$ \\
\hline 3570 & Synthetic material (nylon) & $\mathrm{D}$ & $\mathrm{CH}$ \\
\hline 3571 & Synthetic material (nylon) & $\mathrm{D}$ & $\mathrm{CH}$ \\
\hline 3572 & Synthetic material (nylon) & D & $\mathrm{CH}$ \\
\hline 3573 & Synthetic material (nylon) & $\mathrm{D}$ & $\mathrm{CH}$ \\
\hline 3574 & Synthetic material (nylon) & $\mathrm{D}$ & $\mathrm{CH}$ \\
\hline 3575 & Synthetic material (nylon) & D & $\mathrm{CH}$ \\
\hline 3576 & Synthetic material (nylon) & $\mathrm{D}$ & $\mathrm{CH}$ \\
\hline 3577 & Steel shots & $\mathrm{D}$ & $\mathrm{CH}$ \\
\hline 3578 & Steel shots & D & $\mathrm{CH}$ \\
\hline 3579 & Steel shots & $\mathrm{D}$ & $\mathrm{CH}$ \\
\hline 3580 & Steel shots & $\mathrm{D}$ & $\mathrm{CH}$ \\
\hline 3581 & Steel shots & D & $\mathrm{CH}$ \\
\hline 3582 & Steel shots & $\mathrm{D}$ & $\mathrm{CH}$ \\
\hline 3583 & Steel shots & $\mathrm{D}$ & $\mathrm{CH}$ \\
\hline 3584 & Steel shots & $\mathrm{D}$ & $\mathrm{CH}$ \\
\hline 3585 & Steel shots & $\mathrm{D}$ & $\mathrm{CH}$ \\
\hline 3586 & Steel shots & $\mathrm{D}$ & $\mathrm{CH}$ \\
\hline 3587 & Steel shots & $\mathrm{D}$ & $\mathrm{CH}$ \\
\hline 3588 & Steel shots & D & $\mathrm{CH}$ \\
\hline 3589 & Steel shots & $\mathrm{D}$ & $\mathrm{CH}$ \\
\hline 3590 & Steel shots & D & $\mathrm{CH}$ \\
\hline 3591 & Steel shots & D & $\mathrm{CH}$ \\
\hline 3592 & Steel shots & $\mathrm{D}$ & $\mathrm{CH}$ \\
\hline 3593 & Steel shots & $\mathrm{D}$ & $\mathrm{CH}$ \\
\hline 3594 & Steel shots & D & $\mathrm{CH}$ \\
\hline 3595 & Steel shots & $\mathrm{D}$ & $\mathrm{CH}$ \\
\hline 3596 & Steel shots & D & $\mathrm{CH}$ \\
\hline 3597 & Steel shots & D & $\mathrm{CH}$ \\
\hline 3598 & Steel shots & $\mathrm{D}$ & $\mathrm{CH}$ \\
\hline 3599 & Steel shots & D & $\mathrm{CH}$ \\
\hline 3600 & Steel shots & D & $\mathrm{CH}$ \\
\hline 3601 & Steel shots & D & $\mathrm{CH}$ \\
\hline 3602 & Steel shots & D & $\mathrm{CH}$ \\
\hline 3603 & Steel shots & D & $\mathrm{CH}$ \\
\hline 3604 & Steel shots & D & $\mathrm{CH}$ \\
\hline 3605 & Steel shots & D & $\mathrm{CH}$ \\
\hline 3606 & Steel shots & D & $\mathrm{CH}$ \\
\hline 3607 & Steel shots & D & $\mathrm{CH}$ \\
\hline 3608 & Steel shots & D & $\mathrm{CH}$ \\
\hline 3609 & Steel shots & D & $\mathrm{CH}$ \\
\hline 3610 & Steel shots & D & $\mathrm{CH}$ \\
\hline 3611 & Steel shots & D & $\mathrm{CH}$ \\
\hline 3612 & Zirconium & B & So \\
\hline 3613 & Zirconium & B & SO \\
\hline 3614 & Zirconium & B & So \\
\hline 3615 & Zirconium & B & so \\
\hline 3616 & Zirconium & B & So \\
\hline 3617 & Zirconium & B & So \\
\hline 3618 & Zirconium & D & SO \\
\hline 3619 & Zirconium & D & $\mathrm{CH}$ \\
\hline 3620 & Zirconium & D & $\mathrm{CH}$ \\
\hline 3621 & Zirconium & D & $\mathrm{CH}$ \\
\hline 3622 & Zirconium & D & $\mathrm{CH}$ \\
\hline 3623 & Zirconium & D & $\mathrm{CH}$ \\
\hline 3624 & Zirconium & D & $\mathrm{CH}$ \\
\hline 3625 & Zirconium & D & $\mathrm{CH}$ \\
\hline $\begin{array}{l}3025 \\
3626\end{array}$ & Zirconium & $\begin{array}{l}\mathrm{D} \\
\mathrm{D}\end{array}$ & $\mathrm{CH}$ \\
\hline 3627 & Zirconium & D & $\mathrm{CH}$ \\
\hline 3628 & Zirconium & D & $\mathrm{CH}$ \\
\hline 3629 & Zirconium & D & $\mathrm{CH}$ \\
\hline
\end{tabular}




\section{S17.2 SDC data (this work)}

\section{Source: This work}

\begin{tabular}{llll} 
Nr. & $\begin{array}{l}\text { Terminal } \\
\text { Reynolds } \\
\text { number } \\
\text { Ret }\end{array}$ & $\begin{array}{l}\text { Drag } \\
\text { coefficient }\end{array}$ & Sphericity \\
[\#] & {$[-]$} & CD & phi \\
\hline
\end{tabular}

$\begin{array}{llll}41.39650935 & 1.329689115 & 0.88\end{array}$ $\begin{array}{llll}41.1153756 & 1.347924638 & 0.88 \\ 41.09229532 & 1.349449871 & 0.88\end{array}$ $\begin{array}{llll}40.38459467 & 1.397159862 & 0.88\end{array}$ $\begin{array}{llll}40.38459467 & 1.397159862 & 0.88\end{array}$ $\begin{array}{llll}51.1709284 & 1.27181368 & 0.88\end{array}$ $\begin{array}{llll}49.60889893 & 1.353165477 & 0.88\end{array}$ $\begin{array}{llll}48.08665327 & 1.440193946 & 0.88\end{array}$ $\begin{array}{llll}46.11568167 & 1.565931714 & 0.88\end{array}$ $\begin{array}{llll}46.04309665 & 1.570872858 & 0.88 \\ 73.06264664 & 1.00327585 & 0.88\end{array}$ $\begin{array}{llll}73.06264664 & 1.00327585 & 0.88 \\ 69.80010078 & 1.099256543 & 0.88\end{array}$ $\begin{array}{llll}69.80010078 & 1.099256543 & 0.88 \\ 68.80481106 & 1.131288951 & 0.88\end{array}$ $\begin{array}{llll}67.75467658 & 1.166628564 & 0.88\end{array}$ $\begin{array}{llll}65.86478353 & 1.234538451 & 0.88\end{array}$ $\begin{array}{llll}45.66848896 & 1.835007531 & 0.85\end{array}$ $\begin{array}{llll}53.27990379 & 1.348168798 & 0.85\end{array}$ $\begin{array}{llll}55.11714185 & 1.259788844 & 0.85\end{array}$ $\begin{array}{llll}61.89340227 & 0.999038955 & 0.85\end{array}$ $\begin{array}{llll}48.80601874 & 1.606661441 & 0.85\end{array}$ $\begin{array}{llll}151.2351426 & 0.697306524 & 0.85 \\ 129.9322903 & 0.944702318 & 0.85\end{array}$ $\begin{array}{llll}129.9322903 & 0.944702318 & 0.85\end{array}$ $\begin{array}{llll}118.9956291 & 1.126333885 & 0.85 \\ 94.52196411 & 1.785104701 & 0.85\end{array}$ $\begin{array}{lllll}90.95260323 & 1.927963914 & 0.85\end{array}$ $\begin{array}{llll}140.6086757 & 0.642354767 & 0.89\end{array}$ $\begin{array}{llll}130.4399684 & 0.746410632 & 0.89\end{array}$ $\begin{array}{lllll}127.4730155 & 0.781560619 & 0.89\end{array}$ $\begin{array}{llll}124.9357341 & 0.813627916 & 0.89\end{array}$ $\begin{array}{lll}116.2365052 & 0.93997023 & 0.89\end{array}$ $\begin{array}{lll}113.2991206 & 0.989341232 & 0.89\end{array}$ $\begin{array}{llll}105.5274257 & 1.140429648 & 0.89\end{array}$ $\begin{array}{llll}139.488745 & 0.656253619 & 0.89\end{array}$ $\begin{array}{llll}132.5554375 & 0.726699648 & 0.89\end{array}$ $\begin{array}{lllll}128.1297266 & 0.777768313 & 0.89\end{array}$ $\begin{array}{llll}126.7875135 & 0.794322885 & 0.89\end{array}$ $\begin{array}{llll}126.4817559 & 0.798167928 & 0.89\end{array}$ $\begin{array}{llll}126.2787354 & 0.800736447 & 0.89\end{array}$ $\begin{array}{llll}119.6527029 & 0.89187711 & 0.89\end{array}$ $\begin{array}{llll}116.2921687 & 0.944167627 & 0.89\end{array}$ $\begin{array}{llll}115.7787375 & 0.952560186 & 0.89\end{array}$ $\begin{array}{llll}115.5237183 & 0.956770392 & 0.8\end{array}$ $\begin{array}{llll}114.9330199 & 0.966630309 & 0.89 \\ 135.306192 & 0.701225427 & 0.89\end{array}$ $\begin{array}{llll}135.3069192 & 0.701225427 & 0.89\end{array}$ $\begin{array}{llll}119.9755648 & 0.891891573 & 0.89\end{array}$ $\begin{array}{llll}11 & 12705483 & 1.020328524 & 0.89\end{array}$ $\begin{array}{llll}112.11306807 & 1.021054091 & 0.89\end{array}$ $\begin{array}{llll}122.6302107 & 0.858315808 & 0.89\end{array}$ $\begin{array}{llll}121.406732 & 0.875702364 & 0.89\end{array}$ $\begin{array}{llll}117.9664219 & 0.927524193 & 0.89\end{array}$ $\begin{array}{llll}114.7989636 & 0.97941359 & 0.89\end{array}$ $\begin{array}{llll}114.7989636 & 0.97941359 & 0.89\end{array}$ $\begin{array}{llll}110.779392 & 1.051778056 & 0.89\end{array}$ $\begin{array}{llll}110.3158799 & 1.060635095 & 0.89\end{array}$ $\begin{array}{lll}110.0855754 & 1.065077541 & 0.89 \\ 110.0855754 & 1.065077541 & 0.89\end{array}$ $\begin{array}{llll}104.6249813 & 1.179156021 & 0.89\end{array}$ $\begin{array}{llll}104.624083 & 1.179156021 & 0.89\end{array}$ $\begin{array}{llll}102.0599818 & 1.23917056 & 0.89\end{array}$ $\begin{array}{llll}136.1533184 & 0.700046309 & 0.89\end{array}$ $\begin{array}{llll}134.1951065 & 0.720625909 & 0.89\end{array}$ $\begin{array}{llll}110.8445953 & 1.056219513 & 0.89\end{array}$ $\begin{array}{llll}100.7102323 & 1.279487465 & 0.89\end{array}$ $\begin{array}{llll}155.6792804 & 0.617644919 & 0.8 \\ 152.337328 & 0.64504165 & 0.89\end{array}$ $\begin{array}{llll}152.337328 & 0.645041766 & 0.89\end{array}$ $\begin{array}{lllll}142.6408147 & 0.735720542 & 0.89\end{array}$ $\begin{array}{llll}136.5782941 & 0.802485392 & 0.89 \\ 134.6348875 & 0.825819778 & 0.89\end{array}$ $\begin{array}{llll}133.8939723 & 0.834984573 & 0.89\end{array}$ $\begin{array}{lllll}133.8939723 & 0.834984573 & 0.89\end{array}$ $\begin{array}{lllll}131.0101021 & 0.872149494 & 0.89\end{array}$ $\begin{array}{lllll}130.408218 & 0.880218684 & 0.89\end{array}$ $\begin{array}{llll}129.811839 & 0.888325029 & 0.89\end{array}$ $\begin{array}{llll}128.9274283 & 0.900554217 & 0.89\end{array}$ $\begin{array}{llll}128.5382134 & 0.906016246 & 0.8\end{array}$ $\begin{array}{llll}128.2478409 & 0.910123606 & 0.89\end{array}$ $\begin{array}{llll}127.671014 & 0.918366193 & 0.89\end{array}$ $\begin{array}{llll}126.7210809 & 0.932186408 \quad 0.89\end{array}$ $\begin{array}{lllll}126.1578761 & 0.940528079 & 0.89\end{array}$ $\begin{array}{llll}126.1578761 & 0.940528079 & 0.89\end{array}$ $\begin{array}{llll}122.0882672 & 1.004274982 & 0.89\end{array}$ $\begin{array}{llll}121.6522377 & 1.011486988 & 0.89\end{array}$ $\begin{array}{llll}121.3921117 & 1.015826576 & 0.89\end{array}$ $\begin{array}{llll}120.6183659 & 1.028901078 & 0.89\end{array}$ $\begin{array}{lllll}120.2776361 & 1.034738803 & 0.89\end{array}$ $\begin{array}{llll}120.0233493 & 1.039127934 & 0.8\end{array}$ $\begin{array}{llll}120.0233493 & 1.039127934 & 0.89\end{array}$ $\begin{array}{llll}119.0168642 & 1.05677735 & 0.89\end{array}$ $\begin{array}{llll}118.4375054 & 1.067141479 & 0.89\end{array}$ $\begin{array}{llll}118.4375054 & 1.0671435 & 0.89 & 0.89 \\ 117.5383939 & 1.083530148 & 0.89\end{array}$ $\begin{array}{llll}115.4665307 & 1.122763458 & 0.89\end{array}$ $\begin{array}{llll}115.2321602 & 1.127335276 & 0.89\end{array}$ $\begin{array}{llll}113.7696277 & 1.156505838 & 0.89\end{array}$ $\begin{array}{llll}112.121878 & 1.190747769 & 0.89\end{array}$ $\begin{array}{lll}111.2430651 & 1.20963574 & 0.89\end{array}$ $\begin{array}{lll}110.5929433 & 1.223899255 & 0.89\end{array}$ $\begin{array}{llll}107.5209171 & 1.294835457 & 0.89\end{array}$ $\begin{array}{llll}105.7845545 & 1.337691541 & 0.89\end{array}$ $\begin{array}{llll}101.0700432 & 1.465224052 & 0.89\end{array}$ $\begin{array}{llll}94.40861015 & 1.679489508 & 0.89\end{array}$ $\begin{array}{llll}120.6512339 & 1.127823595 & 0.89\end{array}$ $\begin{array}{llll}177.0023793 & 0.526679361 & 0.89\end{array}$ 


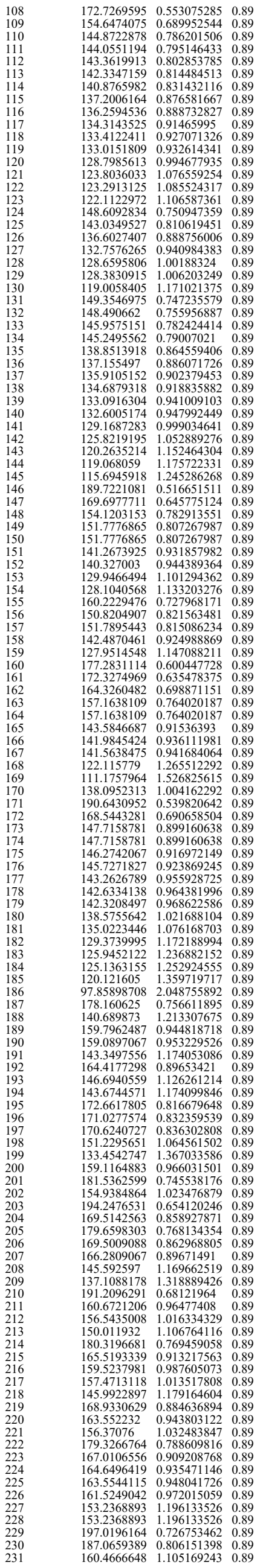




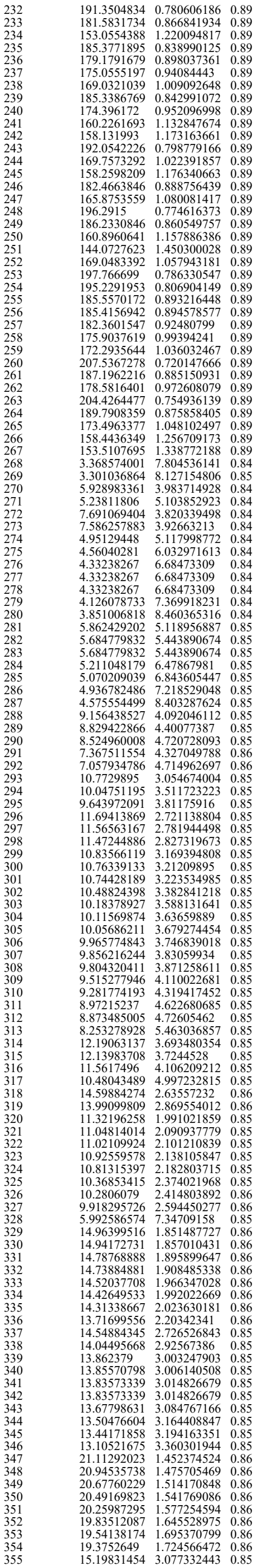




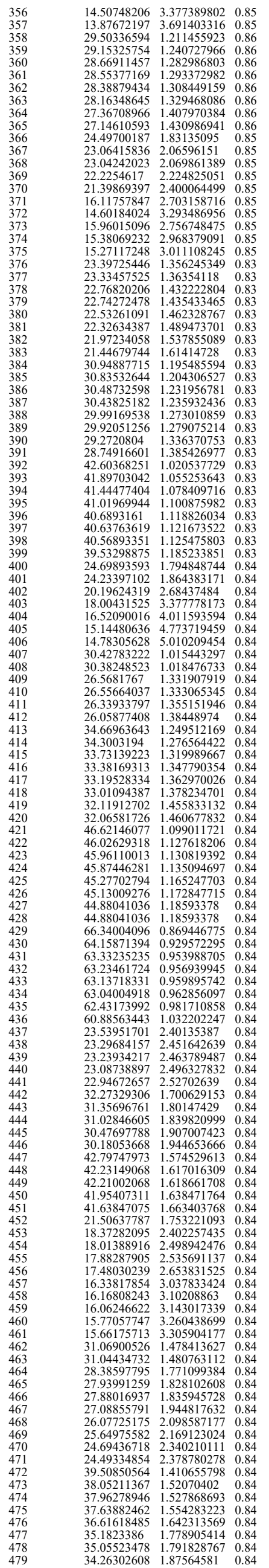




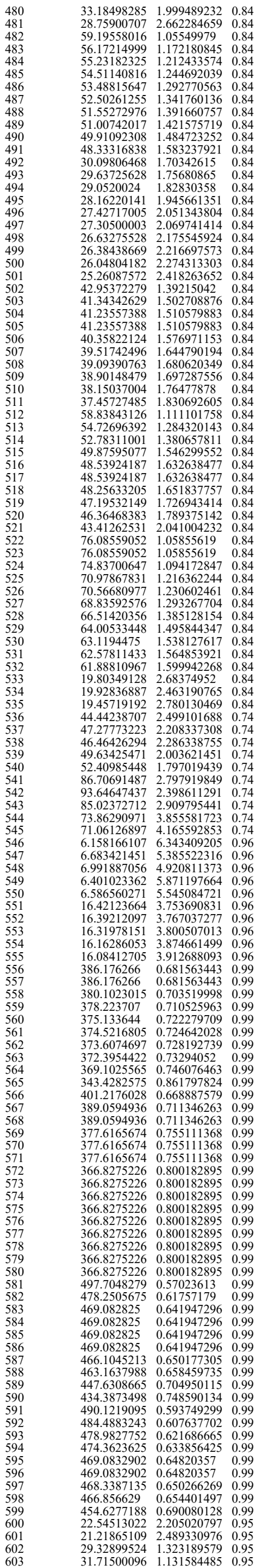




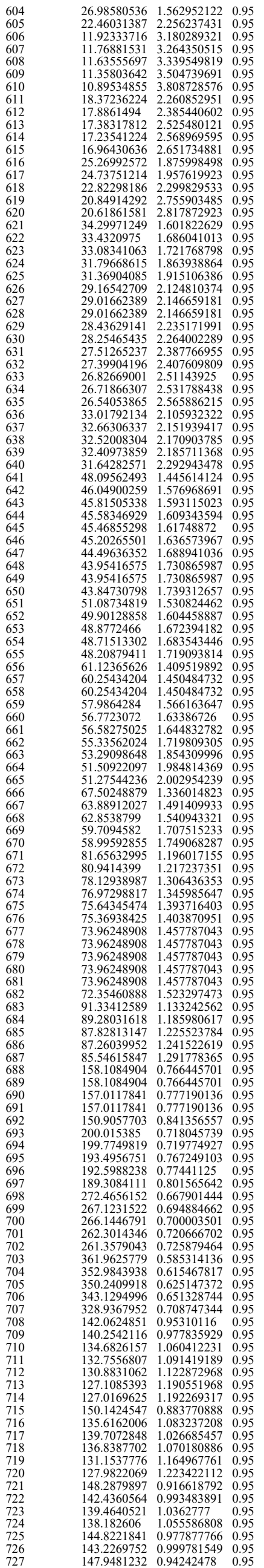




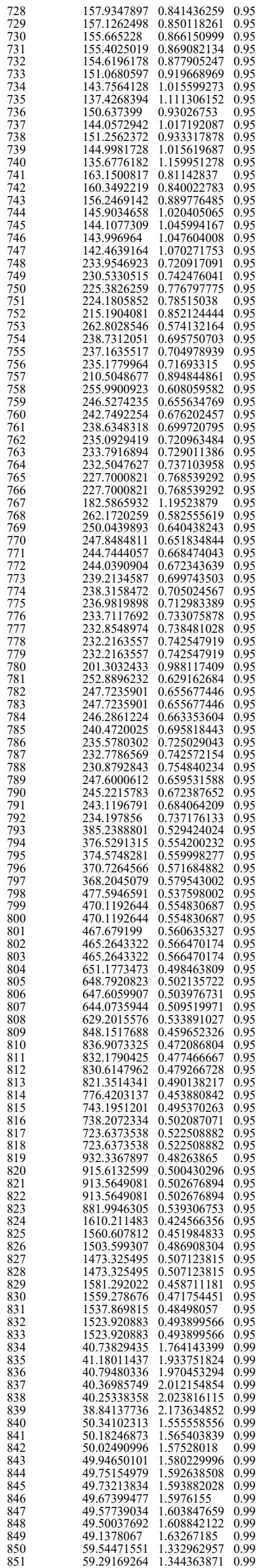




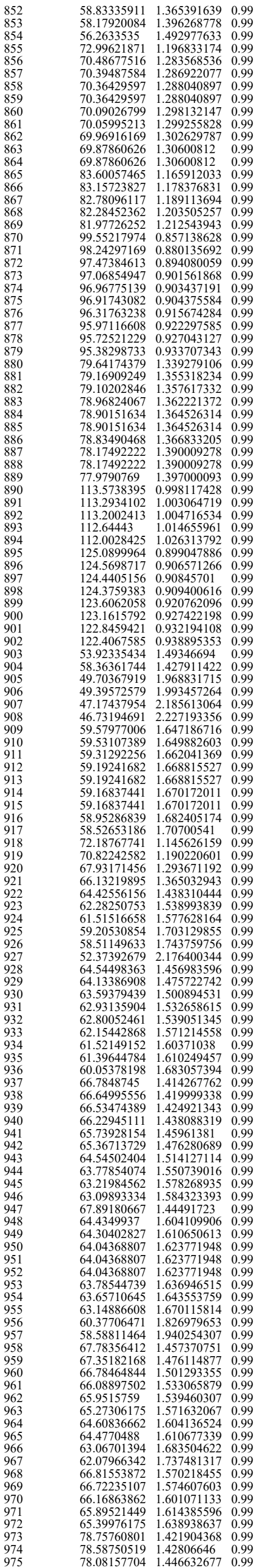




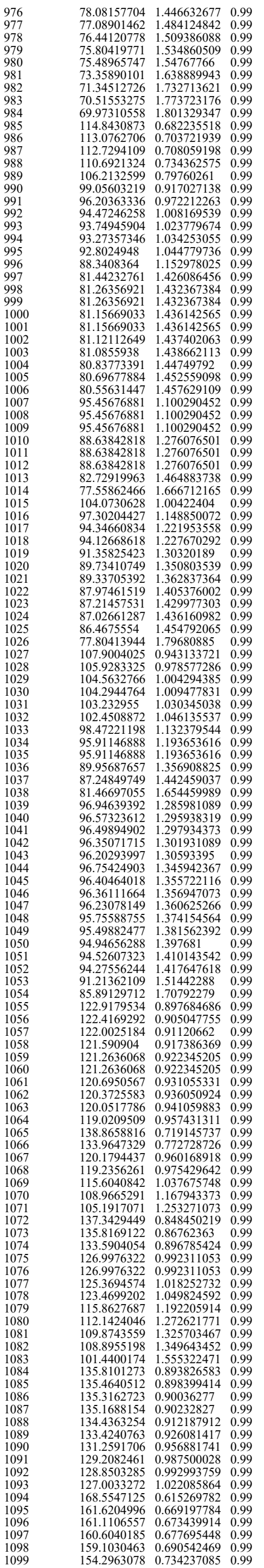


$\begin{array}{llll}148.0350083 & 0.797661294 & 0.99\end{array}$ $\begin{array}{llll}148.0350083 & 0.797661294 & 0.99 \\ 148.0350083 & 0.797661294 & 0.99\end{array}$ $\begin{array}{llll}135.8299943 & 0.947449386 & 0.99\end{array}$ $\begin{array}{llll}132.6547477 & 0.993348837 & 0.99\end{array}$ $\begin{array}{llll}125.1766615 & 1.115579834 & 0.99\end{array}$ $\begin{array}{llll}131.5351108 & 1.103756654 & 0.99\end{array}$ $\begin{array}{llll}130.8785295 & 1.114858914 & 0.99\end{array}$ $\begin{array}{llll}130.1207548 & 1.12788177 & 0.99\end{array}$ $\begin{array}{llll}129.4781831 & 1.139104405 & 0.99\end{array}$ $\begin{array}{llll}129.2654006 & 1.142857629 & 0.99 \\ 152.9376617 & 0.892110776 & 0.99\end{array}$ $\begin{array}{lllll}150.8322423 & 0.917189981 & 0.99\end{array}$ $\begin{array}{llll}150.5007429 & 0.921234916 & 0.99\end{array}$ $\begin{array}{llll}150.0062169 & 0.927319004 & 0.99\end{array}$ $\begin{array}{llll}147.4226868 & 0.960105667 & 0.99\end{array}$ $\begin{array}{llll}146.7906496 & 0.96839133 & 0.99\end{array}$ $\begin{array}{llll}144.6969636 & 0.996618264 & 0.99\end{array}$ $\begin{array}{llll}144.2397852 & 1.002945983 & 0.99\end{array}$ $\begin{array}{llll}144.0880337 & 1.005059673 & 0.99\end{array}$ $\begin{array}{lll}83.99969448 & 1.132662575 & 0.99 \\ 91.17646549 & 1.07677918 & 0.99\end{array}$ $\begin{array}{llll}91.17646549 & 1.07677918 & 0.99\end{array}$ $\begin{array}{llll}90.43183011 & 1.094585056 & 0.99\end{array}$ $87.1117804 \quad 1.179609807 \quad 0.99$ $\begin{array}{llll}86.111780987 & 1.179824311 & 0.99\end{array}$ $\begin{array}{llll}106.5480821 & 0.947889261 & 0.99\end{array}$ $\begin{array}{llll}102.934241 & 1.015615086 & 0.99\end{array}$ $\begin{array}{llll}99.99487768 & 1.076200945 & 0.99\end{array}$ $\begin{array}{llll}99.88517402 & 1.078566221 & 0.99\end{array}$ $\begin{array}{llll}99.39447175 & 1.089242093 & 0.99\end{array}$ $\begin{array}{llll}99.07000666 & 1.09638855 & 0.99\end{array}$ $\begin{array}{llll}98.53391355 & 1.108351239 & 0.99\end{array}$ $\begin{array}{llll}98.26803683 & 1.114356924 & 0.99\end{array}$ $96.75381097 \quad 1.1495098990 .99$ $\begin{array}{llll}114.9998214 & 0.975514042 & 0.99\end{array}$ $\begin{array}{lll}114.3401666 & 0.986802442 & 0.99\end{array}$ $\begin{array}{llll}112.9365574 & 1.011483417 & 0.99\end{array}$ $\begin{array}{llll}112.1949477 & 1.02489944 & 0.99\end{array}$ $\begin{array}{lll}112.1949477 & 1.02489944 & 0.99\end{array}$ $\begin{array}{llll}140.0714366 & 0.887305289 & 0.99\end{array}$ $\begin{array}{llll}139.9866989 & 0.888379833 & 0.99\end{array}$ $\begin{array}{llll}139.4804179 & 0.894840756 & 0.99\end{array}$ $\begin{array}{llll}139.2286482 & 0.898079996 & 0.99\end{array}$ $\begin{array}{llll}139.1449268 & 0.899161043 & 0.99\end{array}$ $\begin{array}{llll}138.9777858 & 0.901325088 & 0.99\end{array}$ $\begin{array}{llll}38.7278257 & 0.904576033 & 0.99\end{array}$ $\begin{array}{llll}138.5616846 & 0.90674658 & 0.99\end{array}$ $\begin{array}{lll}138.4787632 & 0.90783283 & 0.99\end{array}$ $\begin{array}{llll}164.0280253 & 0.826807637 & 0.99\end{array}$ $\begin{array}{llll}163.8566271 & 0.828538264 & 0.99\end{array}$ $\begin{array}{llll}163.0049815 & 0.837218543 & 0.99\end{array}$ $\begin{array}{llll}162.4982302 & 0.842448422 & 0.99\end{array}$ $\begin{array}{llll}170.1745135 & 0.802024047 & 0.99\end{array}$ $\begin{array}{llll}169.9362049 & 0.803046711 & 0.99\end{array}$ $\begin{array}{llll}169.1818007 & 0.8102236 & 0.99\end{array}$ $\begin{array}{llll}69.0746779 & 0.811251477 & 0.99\end{array}$ $\begin{array}{llll}168.5406454 & 0.816400632 & 0.99\end{array}$ $\begin{array}{llll}168.5406454 & 0.816400632 & 0.99\end{array}$ $\begin{array}{llll}168.2218422 & 0.819497945 & 0.99\end{array}$ $\begin{array}{llll}167.6931752 & 0.824673163 & 0.99\end{array}$ $\begin{array}{llll}167.3775669 & 0.827786114 & 0.99\end{array}$ $\begin{array}{llll}166.9585993 & 0.831945836 & 0.99\end{array}$ $\begin{array}{llll}223.7144376 & 0.702357894 & 0.99\end{array}$ $\begin{array}{lllll}22.1971564 & 0.78429637 & 0.99\end{array}$ $\begin{array}{llll}220.2071564 & 0.72490924 & 0.99\end{array}$ $\begin{array}{llll}217.2872825 & 0.744522584 & 0.99\end{array}$ $\begin{array}{llll}238.8289513 & 0.668061722 & 0.99\end{array}$ $\begin{array}{lll}234.3970739 & 0.693563389 & 0.99\end{array}$ $\begin{array}{llll}234.0753209 & 0.695471403 & 0.99\end{array}$ $\begin{array}{lll}233.75445 & 0.697382038 & 0.99\end{array}$ $\begin{array}{llll}233.5943442 & 0.698338338 & 0.99\end{array}$ $\begin{array}{llll}233.2747897 & 0.700252904 & 0.99\end{array}$ $\begin{array}{lllll}233.11534 & 0.701252117 & 0.99\end{array}$ $\begin{array}{llll}232.9561083 & 0.702170091 & 0.99\end{array}$ $\begin{array}{lllll}230.5934702 & 0.716632535 & 0.99\end{array}$ $\begin{array}{lllll}229.8165381 & 0.721486111 & 0.99\end{array}$ $\begin{array}{llll}188.327315 & 0.748240875 & 0.99\end{array}$ $\begin{array}{llll}199.5778574 & 0.746241708 & 0.99\end{array}$ $\begin{array}{llll}197.7299142 & 0.760255331 & 0.99\end{array}$ $\begin{array}{llll}190.1829709 & 0.821790233 & 0.99\end{array}$ $\begin{array}{lll}188.980803 & 0.832278834 & 0.99\end{array}$ $\begin{array}{llll}181.6328252 & 0.900980836 & 0.99\end{array}$ $\begin{array}{llll}210.7664624 & 0.701185412 & 0.99\end{array}$ $\begin{array}{llll}210.4827928 & 0.703076673 & 0.99\end{array}$ $\begin{array}{llll}208.2406326 & 0.71829846 & 0.99\end{array}$ $\begin{array}{lll}207.137371 & 0.725970485 & 0.99\end{array}$ $\begin{array}{llll}205.7746251 & 0.735617828 & 0.99\end{array}$ $\begin{array}{llll}203.6311394 & 0.751186028 & 0.99\end{array}$ $\begin{array}{llll}203.1022274 & 0.755103549 & 0.99\end{array}$ $\begin{array}{llll}201.0137726 & 0.770875522 & 0.99\end{array}$ $\begin{array}{llll}199.2212931 & 0.784809723 & 0.99\end{array}$ $\begin{array}{llll}198.9678309 & 0.786810512 & 0.99\end{array}$ $\begin{array}{llll}195.7305571 & 0.813052557 & 0.99\end{array}$ $\begin{array}{llll}220.3639223 & 0.731705346 & 0.99\end{array}$ $\begin{array}{llll}218.7450384 & 0.745982187 & 0.99\end{array}$ $\begin{array}{llll}217.3741484 & 0.751971582 & 0.99\end{array}$ $\begin{array}{llll}216.6824248 & 0.756780341 & 0.99\end{array}$ $\begin{array}{llll}215.1420284 & 0.767656087 & 0.99\end{array}$ $\begin{array}{lll}214.9722241 & 0.768869292 & 0.99\end{array}$ $\begin{array}{llll}213.6233788 & 0.778609424 & 0.99\end{array}$ $\begin{array}{llll}213.6233788 & 0.778609424 & 0.99\end{array}$ $\begin{array}{llll}262.2563899 & 0.570017496 & 0.99\end{array}$ $\begin{array}{lll}245.00268 & 0.653128558 & 0.99\end{array}$ $\begin{array}{llll}236.6975044 & 0.699766239 & 0.99\end{array}$ $\begin{array}{llll}221.2301427 & 0.801035465 & 0.99\end{array}$ $\begin{array}{llll}262315041 & 0.605423774 & 0.99\end{array}$ $\begin{array}{llll}255.3354833 & 0.638974482 & 0.99\end{array}$ $\begin{array}{llll}244.4932123 & 0.696902857 & 0.99\end{array}$ $\begin{array}{llll}241.417826 & 0.714771436 & 0.99\end{array}$ $\begin{array}{llll}241.417826 & 0.714771436 & 0.99\end{array}$ $\begin{array}{llll}254.3727958 & 0.651457747 & 0.99\end{array}$ $\begin{array}{llll}253.6632482 & 0.655107363 & 0.99\end{array}$ $\begin{array}{llll}251.210703 & 0.6579613 & 0.99\end{array}$ $\begin{array}{llll}249.8304244 & 0.675362477 & 0.99\end{array}$ 


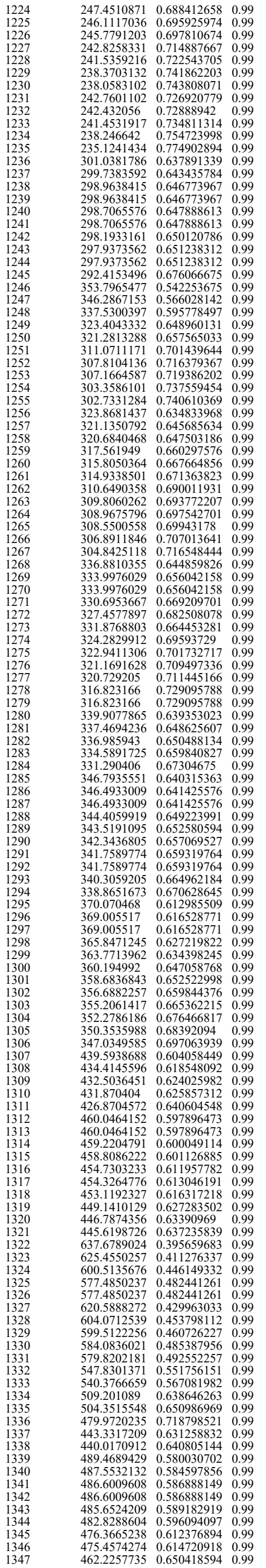


$\begin{array}{llll}457.9773749 & 0.662541699 & 0.99\end{array}$ $\begin{array}{llll}561.72868107 & 0.517205984 & 0.99\end{array}$ $\begin{array}{llll}559.0513299 & 0.531508932 & 0.99\end{array}$ $\begin{array}{llll}556.3108822 & 0.536758371 & 0.99\end{array}$ $\begin{array}{llll}554.2731134 & 0.540712379 & 0.99\end{array}$ $\begin{array}{llll}548.911342 & 0.551327338 & 0.99\end{array}$ $\begin{array}{llll}546.2691695 & 0.556673511 & 0.99\end{array}$ $\begin{array}{llll}545.612596 & 0.558014085 & 0.99\end{array}$ $\begin{array}{llll}545.612596 & 0.558014085 & 0.99\end{array}$ $\begin{array}{llll}615.2253822 & 0.529138292 & 0.99\end{array}$ $610.2031342 \quad 0.537884231 \quad 0.99$ $\begin{array}{llll}610.2031342 & 0.537884231 & 0.99 \\ 607.7226336 & 0.542284084 & 0.99\end{array}$ $\begin{array}{lllll}604.0394662 & 0.548917467 & 0.99\end{array}$ $\begin{array}{llll}602.8216447 & 0.551137555 & 0.99\end{array}$ $\begin{array}{llll}600.4006742 & 0.555591174 & 0.99\end{array}$ $\begin{array}{llll}600.4006742 & 0.555591174 & 0.99\end{array}$ $\begin{array}{llll}595.6166051 & 0.564552178 & 0.99\end{array}$ $\begin{array}{llll}595.6166051 & 0.564552178 & 0.99\end{array}$ $\begin{array}{llll}776.5034004 & 0.448225768 & 0.99\end{array}$ $\begin{array}{llll}776.2347922 & 0.459120797 & 0.99\end{array}$ $\begin{array}{llll}758.1848405 & 0.470146055 & 0.99\end{array}$ $\begin{array}{llll}750.3178123 & 0.480057247 & 0.99\end{array}$ $\begin{array}{llll}747.4096037 & 0.483800369 & 0.99\end{array}$ $\begin{array}{lllll}747.4096037 & 0.483800369 & 0.99\end{array}$ $\begin{array}{llll}746.4452042 & 0.485051307 & 0.99\end{array}$ $\begin{array}{llll}743.5668808 & 0.488813811 & 0.99\end{array}$ $\begin{array}{llll}707.2066422 & 0.540369566 & 0.99\end{array}$ $\begin{array}{llll}889.8519229 & 0.396844575 & 0.99\end{array}$ $\begin{array}{llll}867.7634 & 0.417304701 & 0.99\end{array}$ $\begin{array}{llll}852.6455707 & 0.432233928 & 0.99\end{array}$ $\begin{array}{llll}852.6455707 & 0.43233928 & 0.99\end{array}$ $\begin{array}{llll}872.6610682 & 0.399094434 & 0.99\end{array}$ $\begin{array}{lllll}863.6179484 & 0.407496192 & 0.99\end{array}$ $\begin{array}{llll}835.4800202 & 0.435406328 & 0.99\end{array}$ $\begin{array}{llll}825.139921 & 0.446387141 & 0.99\end{array}$ $\begin{array}{llll}817.0503139 & 0.455270249 & 0.99\end{array}$ $\begin{array}{llll}809.1177866 & 0.464240876 & 0.99\end{array}$ $\begin{array}{llll}809.1177866 & 0.464240876 & 0.99\end{array}$ $\begin{array}{lll}805.209005 & 0.468759009 & 0.99\end{array}$ $\begin{array}{llll}803.2687423 & 0.47102628 & 0.99\end{array}$ $\begin{array}{llll}799.4161344 & 0.475577232 & 0.99\end{array}$ $\begin{array}{llll}799.4161344 & 0.475577232 & 0.99\end{array}$ $\begin{array}{llll}795.6003056 & 0.480150064 & 0.99\end{array}$ $\begin{array}{llll}791.8207317 & 0.484744775 & 0.99\end{array}$ $\begin{array}{llll}789.9443793 & 0.487050335 & 0.99\end{array}$ $\begin{array}{llll}789.9443793 & 0.487050335 & 0.99\end{array}$ $\begin{array}{llll}788.0768985 & 0.489361366 & 0.99\end{array}$ $\begin{array}{llll}786.2182266 & 0.491677866 & 0.99\end{array}$ $\begin{array}{llll}782.5270612 & 0.496327276 & 0.99\end{array}$ $\begin{array}{llll}782.5270612 & 0.496327276 & 0.99\end{array}$ $\begin{array}{lllll}780.694451 & 0.498660186 & 0.99\end{array}$ $\begin{array}{llll}775.6944451 & 0.49860186 & 0.99\end{array}$ $\begin{array}{llll}773.449021 & 0.508046525 & 0.99\end{array}$ $\begin{array}{llll}773.449021 & 0.508046525 & 0.99\end{array}$ $\begin{array}{llll}769.8765082 & 0.512772514 & 0.99\end{array}$ $\begin{array}{llll}769.8765082 & 0.512772514 & 0.99\end{array}$ $\begin{array}{llll}769.8765082 & 0.512772514 & 0.99\end{array}$ $\begin{array}{llll}766.3368461 & 0.517520382 & 0.99\end{array}$ $\begin{array}{llll}766.3368461 & 0.517520382 & 0.99\end{array}$ $\begin{array}{llll}766.3368461 & 0.517520382 & 0.99\end{array}$ $\begin{array}{llll}762.8295837 & 0.52229013 & 0.99\end{array}$ $\begin{array}{llll}761.0879636 & 0.52463209 & 0.99\end{array}$ $\begin{array}{lllll}759.3542781 & 0.527081758 & 0.99\end{array}$ $\begin{array}{llll}759.3542781 & 0.527081758 & 0.99\end{array}$ $\begin{array}{llll}757.6284729 & 0.529485776 & 0.99\end{array}$ $\begin{array}{llll}755.9104945 & 0.531895265 & 0.99\end{array}$ $\begin{array}{llll}754.2002897 & 0.534310223 & 0.99\end{array}$ $\begin{array}{llll}752.497806 & 0.536730651 & 0.99\end{array}$ $\begin{array}{llll}750.8029911 & 0.539156549 & 0.99\end{array}$ $\begin{array}{llll}750.8029911 & 0.539156549 & 0.99\end{array}$ $\begin{array}{lllll}750.8029911 & 0.539156549 & 0.99\end{array}$ $\begin{array}{lllll}749.1157934 & 0.541587917 & 0.99 \\ 747.4361616 & 0.544024755 & 0.99\end{array}$ $\begin{array}{lllll}742.442156 & 0.551368089 & 0.99\end{array}$ $\begin{array}{lllll}740.7922846 & 0.553826806 & 0.99\end{array}$ $\begin{array}{llll}739.1497296 & 0.556290994 & 0.99\end{array}$ $\begin{array}{llll}739.1497296 & 0.556290994 & 0.99\end{array}$ $\begin{array}{lll}737.5144426 & 0.558760651 & 0.99\end{array}$ $\begin{array}{lll}732.65171 & 0.566202442 & 0.99\end{array}$ $\begin{array}{llll}731.0450177 & 0.568693979 & 0.99\end{array}$ $\begin{array}{llll}726.2669457 & 0.576201409 & 0.99\end{array}$ $\begin{array}{llll}723.1161129 & 0.581233712 & 0.99\end{array}$ $\begin{array}{lllll}719.9925012 & 0.586287894 & 0.99\end{array}$ $\begin{array}{llll}713.857593 & 0.591363956 & 0.99\end{array}$ $\begin{array}{llll}712.3002736 & 0.599019073 & 0.99\end{array}$ $\begin{array}{llll}697.3985943 & 0.624891674 & 0.99\end{array}$ $\begin{array}{llll}786.1787168 & 0.52077214 & 0.99\end{array}$ $\begin{array}{llll}761.9886025 & 0.554361878 & 0.99\end{array}$ $\begin{array}{llll}761.9886025 & 0.554361878 & 0.99\end{array}$ $\begin{array}{llll}755.0191945 & 0.564643486 & 0.99\end{array}$ $\begin{array}{llll}755.0191945 & 0.564643486 & 0.99\end{array}$ $\begin{array}{lll}752.7243034 & 0.568091683 & 0.99\end{array}$ $\begin{array}{llll}750.4433206 & 0.571550376 & 0.99\end{array}$ $\begin{array}{llll}737.042547 & 0.592522968 & 0.99\end{array}$ $\begin{array}{llll}728.3714582 & 0.606714632 & 0.99\end{array}$ $\begin{array}{lllll}827.4517696 & 0.472371497 & 0.99\end{array}$ $\begin{array}{llll}824.7027604 & 0.475525889 & 0.99\end{array}$ $\begin{array}{llll}824.7027604 & 0.475525889 & 0.99\end{array}$ $\begin{array}{llll}819.2591779 & 0.481866164 & 0.99\end{array}$ $\begin{array}{llll}788.0493044 & 0.520789575 & 0.99\end{array}$ $\begin{array}{llll}783.0773845 & 0.52742377 & 0.99\end{array}$ $\begin{array}{llll}766.159046 & 0.550974114 & 0.99\end{array}$ $\begin{array}{llll}754.5152915 & 0.568110702 & 0.99\end{array}$ $\begin{array}{llll}752.2288815 & 0.571569511 & 0.99\end{array}$ $\begin{array}{llll}847.384436 & 0.495021602 & 0.99\end{array}$ $\begin{array}{llll}812.9594433 & 0.53783289 & 0.99\end{array}$ $\begin{array}{llll}810.4268593 & 0.541199597 & 0.99\end{array}$ $\begin{array}{llll}810.4268593 & 0.541199597 & 0.99\end{array}$ $\begin{array}{llll}810.4268593 & 0.541199597 & 0.99\end{array}$ $\begin{array}{llll}781.2222878 & 0.582419446 & 0.99\end{array}$ $\begin{array}{llll}778.8832989 & 0.585922713 & 0.99\end{array}$ $\begin{array}{llll}751.8700053 & 0.628781272 & 0.99\end{array}$ $\begin{array}{llll}749.703233 & 0.632421098 & 0.99 \\ 843.068775 & 0.485878593 & 0.99\end{array}$ 
$\begin{array}{llll}842.2199734 & 0.519309523 & 0.99\end{array}$ $\begin{array}{llll}1080.466982 & 0.467457326 & 0.99\end{array}$ $\begin{array}{llll}1071.051147 & 0.475712483 & 0.99\end{array}$ $\begin{array}{lll}1066.404505 & 0.479867157 & 0.99\end{array}$ $\begin{array}{llll}1066.404505 & 0.479867157 & 0.99 \\ 1064.09627 & 0.481951269 & 0.99\end{array}$ $\begin{array}{llll}1059.509648 & 0.486133039 & 0.99\end{array}$ $\begin{array}{lllll}1045.983993 & 0.498786732 & 0.99\end{array}$ $\begin{array}{llll}1043.76322 & 0.500911487 & 0.99\end{array}$ $\begin{array}{llll}1022.063361 & 0.522407411 & 0.99\end{array}$ $\begin{array}{lll}1011.548306 & 0.533324721 & 0.99\end{array}$ $\begin{array}{lll}1200.872333 & 0.41021972 & 0.99\end{array}$ $\begin{array}{lll}1174.406827 & 0.42891682 & 0.99\end{array}$ $\begin{array}{llll}1158.450212 & 0.440814087 & 0.99 \\ 1153.747437 & 0.44441504 & 0.99\end{array}$ $\begin{array}{llll}1153.747437 & 0.444415004 & 0.99\end{array}$ $\begin{array}{llll}1127.803381 & 0.465096881 & 0.99 \\ 1126.313549 & 0.46632811 & 0.99\end{array}$ $\begin{array}{llll}1121.867574 & 0.470031561 & 0.99\end{array}$ $\begin{array}{llll}1121.867574 & 0.47032815 & 0.99 & 0.99\end{array}$ $\begin{array}{llll}1105.861681 & 0.483736204 & 0.99\end{array}$ $\begin{array}{llll}1100.154008 & 0.488768536 & 0.99\end{array}$ $\begin{array}{llll}807.8115026 & 0.499614409 & 0.99\end{array}$ $\begin{array}{llll}790.9820963 & 0.521100768 & 0.99\end{array}$ $\begin{array}{lll}790.9820963 & 0.521100768 & 0.99\end{array}$ $\begin{array}{llll}790.9820963 & 0.521100768 & 0.99\end{array}$ $\begin{array}{llll}774.8396045 & 0.543039473 & 0.99\end{array}$ $\begin{array}{llll}1322.752858 & 0.310343215 & 0.99 \\ 1249.579296 & 0.347753917 & 0.99\end{array}$ $\begin{array}{llll}1249.579296 & 0.347753917 & 0.99\end{array}$ $\begin{array}{llll}1249.579296 & 0.347753917 & 0.99\end{array}$ $\begin{array}{llll}1249.579296 & 0.347753917 & 0.99\end{array}$ $\begin{array}{llll}1208.440883 & 0.37183379 & 0.99\end{array}$ $\begin{array}{llll}1320.780244 & 0.318840086 & 0.99\end{array}$ $\begin{array}{lll}1259.218453 & 0.350777628 & 0.99\end{array}$ $\begin{array}{llll}1142.982904 & 0.425749922 & 0.99\end{array}$ $\begin{array}{llll}1129.945076 & 0.435631603 & 0.99\end{array}$ $\begin{array}{llll}1108.864011 & 0.452352994 & 0.99\end{array}$ $\begin{array}{llll}1100.650204 & 0.459129724 & 0.99\end{array}$ $\begin{array}{llll}1100.650204 & 0.45129724 & 0.99\end{array}$ $\begin{array}{llll}1100.650204 & 0.459129724 & 0.99\end{array}$ $\begin{array}{llll}1042.721245 & 0.511561204 & 0.99\end{array}$ $\begin{array}{llll}1059.138265 & 0.498204313 & 0.99\end{array}$ $\begin{array}{llll}1059.138265 & 0.498204313 & 0.99\end{array}$ $\begin{array}{llll}1045.202235 & 0.511578331 & 0.99\end{array}$ $\begin{array}{lll}1005.511011 & 0.552763221 & 0.99\end{array}$ $\begin{array}{llll}984.7359903 & 0.57633259 & 0.99\end{array}$ $\begin{array}{llll}976.6643838 & 0.585898113 & 0.99\end{array}$ $\begin{array}{llll}960.9117325 & 0.605265344 & 0.99\end{array}$ $\begin{array}{llll}906.4267317 & 0.61999743 & 0.99\end{array}$ $\begin{array}{llll}899.2683383 & 0.69108931 & 0.99\end{array}$ $\begin{array}{lllll}1191.513077 & 0.432644541 & 0.99\end{array}$ $\begin{array}{llll}1131.074008 & 0.480116698 & 0.99\end{array}$ $\begin{array}{lll}131.074008 & 0.480116698 & 0.99\end{array}$ $\begin{array}{llll}1103.096912 & 0.504779277 & 0.99\end{array}$ $\begin{array}{llll}1083.945924 & 0.522773569 & 0.99\end{array}$ $\begin{array}{llll}1076.470435 & 0.530059524 & 0.99\end{array}$ $\begin{array}{lll}1072.771224 & 0.533721409 & 0.99\end{array}$ $\begin{array}{llll}1061.824579 & 0.544782699 & 0.99\end{array}$ $\begin{array}{llll}994.1924402 & 0.621423886 & 0.99 \\ 1145.70245 & 0.425764176 & 0.99\end{array}$ $\begin{array}{llll}1063.866561 & 0.493785671 & 0.99\end{array}$ $\begin{array}{llll}1107.370398 & 0.455750318 & 0.99\end{array}$ $\begin{array}{llll}041.547682 & 0.515174653 & 0.99\end{array}$ $\begin{array}{llll}1041.547682 & 0.515174653 & 0.99\end{array}$ $\begin{array}{llll}023.651674 & 0.533345209 & 0.99\end{array}$ $\begin{array}{llll}1041.547682 & 0.515174653 & 0.99\end{array}$ $\begin{array}{lll}1095.156754 & 0.465972437 & 0.99\end{array}$ $\begin{array}{llll}1063.866561 & 0.493785671 & 0.99\end{array}$ $\begin{array}{llll}1052.588824 & 0.504423477 & 0.99\end{array}$ $\begin{array}{llll}1041.547682 & 0.515174653 & 0.99\end{array}$ $\begin{array}{llll}1034.314712 & 0.522405086 & 0.99\end{array}$ $\begin{array}{llll}1034314712 & 0.522405086 & 0.99\end{array}$ $\begin{array}{llll}1103.269026 & 0.459145095 & 0.99\end{array}$ $\begin{array}{llll}1063.866561 & 0.493785671 & 0.99\end{array}$ $\begin{array}{llll}1013.206249 & 0.544398702 & 0.99\end{array}$ $\begin{array}{lll}1119.859538 & 0.445641568 & 0.99\end{array}$ $\begin{array}{llll}1119.859538 & 0.445641568 & 0.99\end{array}$ $\begin{array}{llll}1023.651674 & 0.533345209 & 0.99\end{array}$ $\begin{array}{llll}1013.206249 & 0.544398702 & 0.99\end{array}$ $\begin{array}{llll}1034.314712 & 0.522405086 & 0.99\end{array}$ $\begin{array}{llll}103.206249 & 0.544398702 & 0.99\end{array}$ $\begin{array}{llll}1013.3146249 & 0.524398702 & 0.99\end{array}$ $\begin{array}{lllll}1023.651674 & 0.533345209 & 0.99\end{array}$ $\begin{array}{llll}1023.651674 & 0.533345209 & 0.99\end{array}$ $\begin{array}{llll}1023.651674 & 0.533345209 & 0.99\end{array}$ $\begin{array}{lll}1095.156754 & 0.465972437 & 0.99\end{array}$ $\begin{array}{llll}1075.388581 & 0.483261234 & 0.99\end{array}$ $\begin{array}{llll}1107.370398 & 0.455750318 & 0.99\end{array}$ $\begin{array}{lll}786.5699315 & 0.54490869 & 0.99\end{array}$ $\begin{array}{llll}871.9889218 & 0.496606788 & 0.99\end{array}$ $\begin{array}{lllll}826.5297837 & 0.55273577 & 0.99\end{array}$ $\begin{array}{llll}809.2704611 & 0.575651584 & 0.99\end{array}$ $\begin{array}{llll}801.8288936 & 0.587315121 & 0.99\end{array}$ $\begin{array}{llll}951.921334 & 0.449525102 & 0.99\end{array}$ $\begin{array}{llll}943.1072476 & 0.457966704 & 0.99\end{array}$ $\begin{array}{llll}921.7699796 & 0.479414261 & 0.99\end{array}$ $\begin{array}{llll}915.5557999 & 0.485944233 & 0.99\end{array}$ $\begin{array}{llll}913.5029842 & 0.488130706 & 0.99\end{array}$ $\begin{array}{llll}909.4248459 & 0.492518376 & 0.99\end{array}$ $\begin{array}{llll}905.3829577 & 0.496925677 & 0.99\end{array}$ 
$\begin{array}{llll}1015.222926 & 0.437950209 & 0.99\end{array}$ $\begin{array}{llll}1013.603751 & 0.439350529 & 0.99\end{array}$ $\begin{array}{lll}1013.603751 & 0.439350529 & 0.99\end{array}$ $\begin{array}{llll}1008.777066 & 0.443564898 & 0.99\end{array}$ $\begin{array}{llll}1008.777066 & 0.443564898 & 0.99\end{array}$ $\begin{array}{llll}1003.996132 & 0.447799384 & 0.99\end{array}$ $\begin{array}{llll}1002.412542 & 0.449215349 & 0.99\end{array}$ $\begin{array}{llll}997.6916041 & 0.453476657 & 0.99\end{array}$ $\begin{array}{lllll}994.5689387 & 0.456328704 & 0.99\end{array}$ $\begin{array}{lllll}967.3204746 & 0.482399458 & 0.99\end{array}$ $\begin{array}{llll}1073.331667 & 0.472393819 & 0.99\end{array}$ $\begin{array}{llll}1065.138295 & 0.479689368 & 0.99\end{array}$ $\begin{array}{llll}1059.74519 & 0.484584126 & 0.99\end{array}$ $\begin{array}{llll}1046.498375 & 0.496929724 & 0.99\end{array}$ $\begin{array}{llll}1041.291916 & 0.501911444 & 0.99\end{array}$ $\begin{array}{lll}1326.899612 & 0.408873655 & 0.99\end{array}$ $\begin{array}{llll}1324.517386 & 0.410345746 & 0.99 \\ 1317.421758 & 0.414777892 & 0.99\end{array}$ $\begin{array}{llll}1310.401748 & 0.419233844 & 0.99\end{array}$ $\begin{array}{llll}1308.078341 & 0.420724452 & 0.99\end{array}$ $\begin{array}{llll}1292.042354 & 0.431232776 & 0.99\end{array}$ $\begin{array}{llll}1289.783539 & 0.432744546 & 0.99\end{array}$ $\begin{array}{lll}1280.826709 & 0.43881808 & 0.99\end{array}$ $\begin{array}{llll}1278.606905 & 0.440343076 & 0.99\end{array}$ $\begin{array}{lll}1276.394782 & 0.441870718 & 0.99\end{array}$ $\begin{array}{llll}1488.003494 & 0.380224455 & 0.99\end{array}$ $\begin{array}{llll}1469.088195 & 0.390078676 & 0.99\end{array}$ $\begin{array}{llll}1432.664521 & 0.410165318 & 0.99\end{array}$ $\begin{array}{llll}1409.369163 & 0.423836562 & 0.99 \\ 1381.29408 & 0.441240783 & 0.99\end{array}$ $\begin{array}{llll}1364.979583 & 0.451851405 & 0.99\end{array}$ $\begin{array}{llll}1328.370935 & 0.477099782 & 0.99\end{array}$ $\begin{array}{llll}1303.401557 & 0.49555456 & 0.99\end{array}$ $\begin{array}{lll}1225.10535 & 0.560920193 & 0.99\end{array}$ $\begin{array}{lll}1274.650052 & 0.518162538 & 0.99\end{array}$ $\begin{array}{llll}1429.265033 & 0.459375435 & 0.99\end{array}$ $\begin{array}{llll}1418.185459 & 0.466581214 & 0.99\end{array}$ $\begin{array}{llll}1418.185459 & 0.466581214 & 0.99\end{array}$ $\begin{array}{llll}1400.096359 & 0.47871546 & 0.99\end{array}$ $\begin{array}{lll}1365.268091 & 0.503451252 & 0.99 \\ 1540.726718 & 0.421664052 & 0.99\end{array}$ $\begin{array}{llll}1533.223179 & 0.421664052 & 0.99\end{array}$ $\begin{array}{llll}1530.738214 & 0.427184962 & 0.99\end{array}$ $\begin{array}{llll}1525.792372 & 0.429958883 & 0.99\end{array}$ $\begin{array}{lll}1525.792372 & 0.429958883 & 0.99\end{array}$ $\begin{array}{llll}1520.878387 & 0.432741782 & 0.99\end{array}$ $\begin{array}{llll}1515.995952 & 0.435533657 & 0.99\end{array}$ $\begin{array}{lll}1511.144765 & 0.438334509 & 0.99\end{array}$ $\begin{array}{lll}1511.144765 & 0.438334509 & 0.99\end{array}$ $\begin{array}{llll}1501.534941 & 0.443963145 & 0.99 \\ 1911.826298 & 0.405709998 & 0.99\end{array}$ $\begin{array}{llll}1911.826298 & 0.405709998 & 0.99\end{array}$ $\begin{array}{llll}1896.026081 & 0.412500005 & 0.99 \\ 1830.471987 & 0.442574511 & 0.99\end{array}$ $\begin{array}{llll}1830.471987 & 0.442574511 & 0.99\end{array}$ $\begin{array}{llll}1801.721118 & 0.456811927 & 0.99\end{array}$ $\begin{array}{llll}1792.337154 & 0.461607819 & 0.99\end{array}$ $\begin{array}{llll}2036.974571 & 0.387425707 & 0.99\end{array}$ $\begin{array}{llll}2033.504427 & 0.388749107 & 0.99\end{array}$ $\begin{array}{llll}2033.504427 & 0.388749107 & 0.99\end{array}$ $\begin{array}{llll}2026.599488 & 0.391402677 & 0.99\end{array}$ $\begin{array}{llll}2016.329559 & 0.395399955 & 0.99\end{array}$ $\begin{array}{llll}1992.766442 & 0.404805913 & 0.99\end{array}$ $\begin{array}{llll}1966.502634 & 0.415690964 & 0.99\end{array}$ $\begin{array}{llll}1806.5206794 & 0.492747814 & 0.99\end{array}$ $\begin{array}{llll}1806.206794 & 0.492747814 & 0.99\end{array}$ $\begin{array}{llll}1345.7415 & 0.439317146 & 0.99\end{array}$ $\begin{array}{llll}1345.7415 & 0.439317146 & 0.99\end{array}$ $\begin{array}{llll}1305.825439 & 0.466585454 & 0.99\end{array}$ $\begin{array}{lll}1237.649813 & 0.51940471 & 0.99\end{array}$ $\begin{array}{llll}1208.528641 & 0.544737847 & 0.99\end{array}$ $\begin{array}{llll}1709.759153 & 0.440297285 & 0.99\end{array}$ $\begin{array}{llll}1724.82311 & 0.432640087 & 0.99\end{array}$ $\begin{array}{llll}1732.455071 & 0.428836676 & 0.99\end{array}$ $\begin{array}{llll}1737.868742 & 0.436532228 & 0.99\end{array}$ $\begin{array}{llll}1715.299019 & 0.448095495 & 0.99\end{array}$ $\begin{array}{llll}1678.957938 & 0.467703504 & 0.99\end{array}$ $\begin{array}{llll}1630.592894 & 0.495860101 & 0.99\end{array}$ $\begin{array}{llll}1584.936293 & 0.524839647 & 0.99\end{array}$ $\begin{array}{llll}1572.357434 & 0.533270672 & 0.99\end{array}$ $\begin{array}{llll}1566.142582 & 0.537511376 & 0.99\end{array}$ $\begin{array}{llll}1553.859111 & 0.546043169 & 0.99\end{array}$ $\begin{array}{llll}1547.789349 & 0.550334258 & 0.99\end{array}$ $\begin{array}{llll}1472.98912 & 0.607646748 & 0.99 \\ 1690.114253 & 0.463763838 & 0.99\end{array}$ $\begin{array}{lll}1690.114253 & 0.463763838 & 0.99 \\ 1629.443485 & 0.498942393 & 0.99\end{array}$ $\begin{array}{llll}1621.129998 & 0.504072873 & 0.99\end{array}$ $\begin{array}{llll}1596.690852 & 0.519621768 & 0.99\end{array}$ $\begin{array}{llll}1565.228963 & 0.540721028 & 0.99\end{array}$ $\begin{array}{llll}1565.228963 & 0.540721028 & 0.99\end{array}$ $\begin{array}{llll}1534.982993 & 0.562240174 & 0.99\end{array}$ $\begin{array}{llll}1864.680044 & 0.380995954 & 0.99\end{array}$ $\begin{array}{llll}1855.966586 & 0.384581779 & 0.99\end{array}$ $\begin{array}{llll}1765.230442 & 0.425134347 & 0.99\end{array}$ $\begin{array}{llll}1735.977837 & 0.388458208 & 0.99 \\ 1927.014977 & 0.392080176 & 0.99\end{array}$ $\begin{array}{llll}1927.014977 & 0.392080176 & 0.99\end{array}$ $\begin{array}{llll}1874.933491 & 0.414164939 & 0.99\end{array}$ $\begin{array}{lllll}1817.621113 & 0.440695227 & 0.99\end{array}$ $\begin{array}{lll}1763.708623 & 0.468049072 & 0.99\end{array}$ $\begin{array}{llll}1719.98031 & 0.492150708 & 0.99\end{array}$ $\begin{array}{llll}1719.98031 & 0.492150708 & 0.99\end{array}$ $\begin{array}{lll}1632.295039 & 0.546446619 & 0.99\end{array}$ $\begin{array}{llll}4829.251913 & 0.444023366 & 0.99\end{array}$ $\begin{array}{llll}4667.863032 & 0.475257892 & 0.99\end{array}$ $\begin{array}{llll}4562.30834 & 0.497503658 & 0.99\end{array}$ $\begin{array}{lll}4418.001751 & 0.53053468 & 0.99 \\ 4354.43338 & 0.54613781 & 0.99\end{array}$ $\begin{array}{llll}4313.061091 & 0.546665526 & 0.99\end{array}$ $\begin{array}{llll}4313.061091 & 0.556665526 & 0.99 \\ 4136.215762 & 0.605283974 & 0.99\end{array}$ $\begin{array}{llll}4017.253804 & 0.64166301 & 0.99\end{array}$ $\begin{array}{llll}3896.563776 & 0.682027628 & 0.99\end{array}$ $\begin{array}{llll}5165.134226 & 0.631509907 & 0.99\end{array}$ $\begin{array}{llll}5142.178074 & 0.637160974 & 0.99\end{array}$ $\begin{array}{lll}5130.776348 & 0.639995947 & 0.99\end{array}$ $\begin{array}{llll}5119.425073 & 0.642837213 & 0.99\end{array}$ $\begin{array}{llll}5096.87254 & 0.648538624 & 0.99\end{array}$ 
$\begin{array}{lllll}1720 & 4840.962622 & 0.718918953 & 0.99 \\ 1721 & 4466.526452 & 0.780338125 & 0.99\end{array}$ $\begin{array}{llll}4646.546452 & 0.780338125 & 0.99\end{array}$ $\begin{array}{lll}4609.522178 & 0.792924021 & 0.99 \\ 4555.079002 & 0.81991653 & 0.99\end{array}$ $\begin{array}{llll}5866.245206 & 0.66730904 & 0.99\end{array}$ $\begin{array}{llll}5866.245206 & 0.66730904 & 0.99\end{array}$ $\begin{array}{lll}5803.1673 & 0.681894599 & 0.99\end{array}$ $\begin{array}{llll}5753.673336 & 0.693676577 & 0.99\end{array}$ $\begin{array}{llll}5729.241602 & 0.69960541 & 0.99\end{array}$ $\begin{array}{llll}5657.17567 & 0.717543282 & 0.99\end{array}$ $\begin{array}{llll}5586.900196 & 0.735708216 & 0.99 \\ 5575.357014 & 0.738757781 & 0.99\end{array}$ $\begin{array}{llll}5495.871272 & 0.760281336 & 0.99\end{array}$ $\begin{array}{llll}5462.495536 & 0.76960325 & 0.99\end{array}$ $\begin{array}{llll}7446.45726 & 0.665128047 & 0.99\end{array}$ $\begin{array}{llll}7302.949518 & 0.691525288 & 0.99\end{array}$ $\begin{array}{llll}7271.806876 & 0.6974611 & 0.99\end{array}$ $\begin{array}{llll}7240.928715 & 0.703422279 & 0.99\end{array}$ $\begin{array}{llll}7119.994624 & 0.727520662 & 0.99\end{array}$ $\begin{array}{llll}6974.391462 & 0.758214392 & 0.99\end{array}$ $\begin{array}{llll}6974.391462 & 0.758214392 & 0.99\end{array}$ $\begin{array}{lllll}6807.340169 & 0.795883969 & 0.99\end{array}$ $\begin{array}{llll}6648.104142 & 0.834466748 & 0.99\end{array}$ $\begin{array}{llll}6558.610432 & 0.857395091 & 0.99\end{array}$ $\begin{array}{llll}6558.610432 & 0.857395091 & 0.99\end{array}$ $\begin{array}{llll}21.80794639 & 2.026711021 & 0.85\end{array}$ $\begin{array}{llll}21.05713858 & 2.173815396 & 0.85\end{array}$ $\begin{array}{llll}20.37969713 & 2.320736967 & 0.85\end{array}$ $\begin{array}{llll}20.37189479 & 2.322514969 & 0.85\end{array}$ $\begin{array}{llll}19.72984397 & 2.476133577 & 0.85\end{array}$ $\begin{array}{llll}28.32939863 & 1.824485258 & 0.85 \\ 27.9069321 & 1.880142983 & 0.85\end{array}$ $\begin{array}{llll}27.9069321 & 1.880142983 & 0.85\end{array}$ $\begin{array}{lll}27.85945114 & 1.886557113 & 0.85 \\ 27.60116714 & 1.922030059 & 0.85\end{array}$ $26.43162616 \quad 2.095884294 \quad 0.85$ $\begin{array}{llll}26.43162616 & 2.095884294 & 0.85 \\ 15.93354347 & 3.445007663 & 0.85\end{array}$ $\begin{array}{llll}15.51927134 & 3.63138486 & 0.85\end{array}$ $\begin{array}{llll}15.49860653 & 3.641075009 & 0.85\end{array}$ $\begin{array}{llll}15.12599546 & 3.822671689 & 0.85\end{array}$ $\begin{array}{llll}15.03319794 & 3.870010825 & 0.8\end{array}$ $\begin{array}{llll}14.76151364 & 4.013776141 & 0.85\end{array}$ $\begin{array}{llll}14.70092012 & 4.046931896 & 0.85\end{array}$ $\begin{array}{llll}14.69164217 & 4.052044885 & 0.85 \\ 14.54931688 & 4.131708996 & 0.85\end{array}$ $\begin{array}{llll}14.54931688 & 4.131708996 & 0.85 \\ 14.48594089 & 4.167940533 & 0.85\end{array}$ $\begin{array}{llll}14.410313335 & 2.193283867 & 0.85\end{array}$ $\begin{array}{llll}26.73594442 & 2.253942272 & 0.85\end{array}$ $\begin{array}{lllll}26.3126253 & 2.327048771 & 0.85\end{array}$ $\begin{array}{llll}25.46383093 & 2.484770966 & 0.85\end{array}$ $\begin{array}{llll}25.29046885 & 2.518953131 & 0.85\end{array}$ $\begin{array}{llll}35.64074061 & 1.917947865 & 0.85\end{array}$ $\begin{array}{llll}35.2840089 & 1.956925948 & 0.8\end{array}$ $\begin{array}{llll}35.06069239 & 1.981934333 & 0.85 \\ 35.04484933 & 1.98372622 & 0.85\end{array}$ $\begin{array}{llll}35.04484933 & 1.983726722 & 0.85 \\ 35.04484933 & 1.983726722 & 0.85\end{array}$ $\begin{array}{llll}51.07641952 & 1.447302586 & 0.85\end{array}$ $\begin{array}{llll}51.97641952 & 1.447302586 & 0.85 \\ 51.7904204 & 1.457716882 & 0.85\end{array}$ $\begin{array}{llll}51.54448251 & 1.471660687 & 0.85\end{array}$ $\begin{array}{llll}50.58365333 & 1.528099633 & 0.85\end{array}$ $\begin{array}{llll}50.23251343 & 1.549538025 & 0.85\end{array}$ $\begin{array}{llll}41.65427161 & 2.253476156 & 0.85\end{array}$ $\begin{array}{llll}41.46861105 & 2.273699559 & 0.85\end{array}$ $\begin{array}{llll}41.2976878 & 2.292559324 & 0.85\end{array}$ $\begin{array}{llll}40.89573228 & 2.33784696 & 0.85\end{array}$ $\begin{array}{llll}40.05316953 & 2.437239895 & 0.85 \\ 22.5331675 & 2.889441607 & 0.85\end{array}$ $\begin{array}{llll}22.5331675 & 2.889441607 & 0.85 \\ 22.07459146 & 3.010738683 & 0.85\end{array}$ $\begin{array}{lll}2.07495857 & 3.08568695 & 0.85\end{array}$ $\begin{array}{llll}21.55002969 & 3.159094858 & 0.85\end{array}$ $\begin{array}{llll}21.52487401 & 3.166483111 & 0.85\end{array}$ $\begin{array}{llll}28.93762232 & 1.82249723 & 0.90\end{array}$ $\begin{array}{llll}28.90724162 & 1.826330031 & 0.90\end{array}$ $\begin{array}{lll}28.86178998 & 1.832086781 & 0.90\end{array}$ $\begin{array}{lll}28.60690664 & 1.86487947 & 0.90\end{array}$ $\begin{array}{lll}28.28366476 & 1.907748863 & 0.90\end{array}$ $\begin{array}{llll}29.38957238 & 2.084350274 & 0.85 \\ 29.34734598 & 2.09035272 & 0.85\end{array}$ $\begin{array}{llll}29.3473458 & 2.09035272 & 0.85\end{array}$ $\begin{array}{lll}29.0413547 & 2.13463436 & 0.8 \\ 28.79570414 & 2.171210009 & 0.85\end{array}$ $\begin{array}{llll}28.29051635 & 2.249445574 & 0.85\end{array}$ $\begin{array}{llll}28.251536955 & 1.615575755 & 0.85\end{array}$ $\begin{array}{llll}37.81471542 & 1.675999144 & 0.85\end{array}$ $\begin{array}{llll}36.90637239 & 1.75951408 & 0.85\end{array}$ $\begin{array}{llll}35.60488822 & 1.790497955 & 0.85\end{array}$ $\begin{array}{lll}35.21460046 & 1.93263542 & 0.8\end{array}$ $\begin{array}{lll}39.57475638 & 1.725602766 & 0.85\end{array}$ $\begin{array}{llll}38.71709912 & 1.802900286 & 0.85 \\ 37.9149763 & 1.879900916 & 0.85\end{array}$ $\begin{array}{llll}37.9149763 & 1.879990916 & 0.85 \\ 37.81942445 & 1.889502618 & 0.85\end{array}$ $\begin{array}{llll}37.81942445 & 1.889502618 & 0.85 \\ 37.68645811 & 1.902859323 & 0.85\end{array}$ $\begin{array}{llll}37.68645811 & 1.902859323 & 0.85 \\ 51.08799659 & 1.515944373 & 0.85\end{array}$ $\begin{array}{llll}50.65988489 & 1.541674229 & 0.85\end{array}$ $\begin{array}{llll}50.46254533 & 1.553755593 & 0.85\end{array}$ $\begin{array}{llll}49.68832545 & 1.602552582 & 0.85\end{array}$ $\begin{array}{llll}49.09647751 & 1.641422346 & 0.85\end{array}$ $\begin{array}{llll}52.68600333 & 1.479042035 & 0.85\end{array}$ $\begin{array}{llll}52.12108569 & 1.511277164 & 0.85\end{array}$ $\begin{array}{llll}51.56815401 & 1.543859792 & 0.85\end{array}$ $\begin{array}{llll}50.94239594 & 1.582021178 & 0.85\end{array}$ $\begin{array}{llll}50.49675442 & 1.610067545 & 0.85\end{array}$ $\begin{array}{llll}68.5690939 & 1.377792242 & 0.85 \\ 08.23782074 & 1.06193218 & 0.85\end{array}$ $\begin{array}{lllll}68.23784074 & 1.419878716 & 0.85\end{array}$ $\begin{array}{lllll}67.76169357 & 1.439903201 & 0.85\end{array}$ $\begin{array}{llll}67.49676949 & 1.451228593 & 0.85\end{array}$ $\begin{array}{llll}30.56101461 & 2.65800254 & 0.91\end{array}$ $\begin{array}{llll}30.25245916 & 2.712498845 & 0.91\end{array}$ $\begin{array}{lll}29.86864908 & 2.782657582 & 0.91\end{array}$ $\begin{array}{llll}29.54733685 & 2.843506609 & 0.91\end{array}$ $\begin{array}{llll}29.36307729 & 2.879305801 & 0.91\end{array}$ $\begin{array}{llll}38.33028464 & 1.757684784 & 0.91\end{array}$ $\begin{array}{llll}37.80037287 & 1.807311093 & 0.91\end{array}$ $\begin{array}{llll}36.45635961 & 1.843025422 & 0.91\end{array}$ $\begin{array}{llll}35.91759568 & 2.001753365 & 0.91\end{array}$ $\begin{array}{llll}40.2981682 & 1.875940865 & 0.91\end{array}$ $\begin{array}{llll}40.01111276 & 1.902954897 & 0.91\end{array}$ $\begin{array}{llll}38.73754949 & 2.030137561 & 0.91\end{array}$ $\begin{array}{lll}37.93261339 & 2.117211406 & 0.91\end{array}$ $\begin{array}{lll}37.69761527 & 2.143690083 & 0.91\end{array}$ $\begin{array}{llll}51.44766048 & 1.532133896 & 0.91\end{array}$ $\begin{array}{llll}49.92283356 & 1.627157256 & 0.91\end{array}$ 


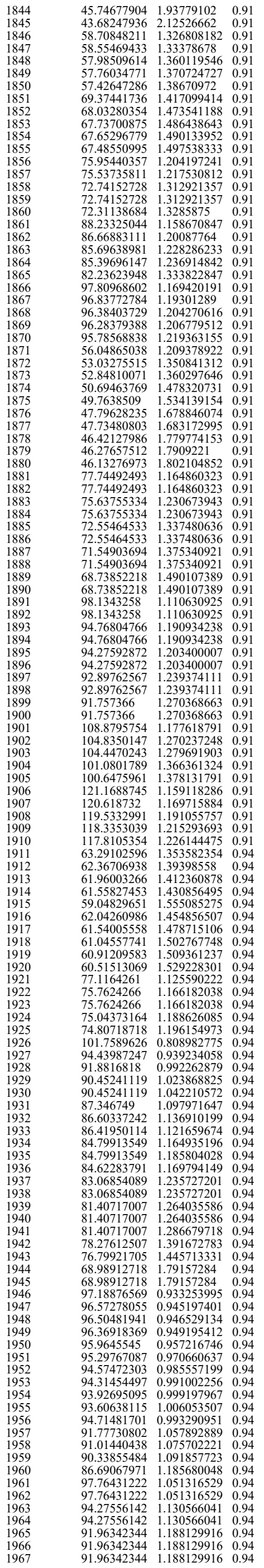




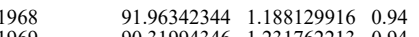
\begin{tabular}{lll}
90.31994346 & 1.231762213 & 0.94 \\
\hline
\end{tabular} $\begin{array}{llll}90.31994346 & 1.231762213 & 0.94 \\ 131.3927501 & 0.876088382 & 0.94\end{array}$ $\begin{array}{llll}131.2716508 & 0.877705526 & 0.94\end{array}$ $\begin{array}{llll}130.1917195 & 0.892326931 & 0.94\end{array}$ $\begin{array}{llll}127.2830573 & 0.93357568 & 0.94\end{array}$ $\begin{array}{lll}126.6042144 & 0.943614056 & 0.94\end{array}$ $\begin{array}{lll}128.3240106 & 0.926983578 & 0.94\end{array}$ $\begin{array}{llll}128.3240106 & 0.926983578 & 0.94\end{array}$ $\begin{array}{lll}122.6060598 & 1.015462799 & 0.94 \\ 122.6060598 & 1.015462799 & 0.94\end{array}$ $\begin{array}{llll}122.5010889 & 1.017203841 \quad 0.94\end{array}$ $\begin{array}{lll}122.5010889 & 1.017203841 & 0.94 \\ 122.5010889 & 1.017203841 & 0.94\end{array}$ $\begin{array}{llll}122.5010889 & 1.017203841 & 0.94\end{array}$ $\begin{array}{llll}122.5010889 & 1.017203841 & 0.94\end{array}$ $\begin{array}{llll}120.5402459 & 1.050566973 & 0.94\end{array}$ $\begin{array}{lll}120.5402459 & 1.050566973 & 0.94\end{array}$ $\begin{array}{lll}135.3241799 & 1.113065503 & 0.94\end{array}$ $\begin{array}{lll}132.6131779 & 1.159039309 & 0.94\end{array}$ $\begin{array}{lll}132.392156 & 1.162912454 & 0.94\end{array}$ $\begin{array}{lll}131.0813425 & 1.186286994 & 0.94 \\ 130.4356216 & 1.198061483 & 0.94\end{array}$ $\begin{array}{lll}130.4356216 & 1.198061483 & 0.94 \\ 152.7016559 & 1.041594439 & 0.94\end{array}$ $149.1792362 \quad 1.091363406 \quad 0.94$ $\begin{array}{llll}149.1792362 & 1.091363406 & 0.94 \\ 147.7970938 & 1.111870883 & 0.94\end{array}$ $\begin{array}{llll}146.9800337 & 1.124266994 & 0.94\end{array}$ $\begin{array}{llll}145.4610911 & 1.147869362 & 0.94\end{array}$ $\begin{array}{llll}89.1578172 & 1.063200403 & 0.94\end{array}$ $\begin{array}{llll}68.45059156 & 1.803763422 & 0.94\end{array}$ $\begin{array}{llll}86.53591402 & 1.135008753 & 0.94\end{array}$ $\begin{array}{llll}82.41274878 & 1.25851303 & 0.94\end{array}$ $\begin{array}{lll}97.77067005 & 0.899249938 & 0.94\end{array}$ $\begin{array}{lll}82.18752941 & 1.27258192 & 0.94\end{array}$ $85.908263 \quad 1.171319473 \quad 0.94$ $\begin{array}{llll}83.70686376 & 1.1733738446 & 0.94\end{array}$ $\begin{array}{lll}83.55020712 & 1.238369302 & 0.94\end{array}$ $\begin{array}{llll}95.32132133 & 0.956773933 & 0.94\end{array}$ $\begin{array}{llll}94.25104685 & 0.978626737 & 0.94\end{array}$ $\begin{array}{llll}90.32127892 & 1.065636986 & 0.94\end{array}$ $\begin{array}{llll}92.95443799 & 1.017483341 & 0.94\end{array}$ $\begin{array}{llll}91.38213694 & 1.052797747 & 0.94\end{array}$ $\begin{array}{llll}88.39188377 & 1.125233847 & 0.94\end{array}$ $\begin{array}{lll}87.70311584 & 1.142977077 & 0.94 \\ 84.414249 & 1.233775222 & 0.94\end{array}$ $\begin{array}{llll}84.414249 & 1.233775222 & 0.94\end{array}$ $\begin{array}{llll}98.28816716 & 0.915164212 & 0.94 \\ 91.63808819 & 1.052808691 & 0.94\end{array}$ $\begin{array}{llll}87.89169003 & 1.144473841 & 0.94\end{array}$ $\begin{array}{llll}97.0142153 & 0.944629008 & 0.94\end{array}$ $\begin{array}{llll}96.12165705 & 0.962253569 & 0.94\end{array}$ $\begin{array}{llll}93.28289932 & 1.021710736 & 0.94\end{array}$ $\begin{array}{llll}92.83656966 & 1.031558493 & 0.94\end{array}$ $\begin{array}{llll}92.0812891 & 1.048550248 & 0.94\end{array}$ $\begin{array}{lll}91.89438526 & 1.052819875 & 0.94\end{array}$ $\begin{array}{llll}90.18585751 & 1.09308806 & 0.94\end{array}$ $\begin{array}{llll}90.00656158 & 1.09744733 & 0.94\end{array}$ $\begin{array}{llll}88.361129223 & 1.138552187 & 0.94\end{array}$ $\begin{array}{llll}85.33785661 & 1.227653181 & 0.94\end{array}$ $\begin{array}{llll}95.54941926 & 0.950066173 & 0.94\end{array}$ $\begin{array}{llll}90.04561778 & 1.115008217 & 0.94\end{array}$ $\begin{array}{llll}87.12429049 & 1.191035546 & 0.94\end{array}$ $\begin{array}{llll}93.49232911 & 1.040083577 & 0.94\end{array}$ $\begin{array}{llll}87.36655946 & 1.191049279 & 0.94\end{array}$ $\begin{array}{llll}109.4901276 & 0.917039789 & 0.94\end{array}$ $\begin{array}{lll}105.9557094 & 0.97924052 & 0.94\end{array}$ $\begin{array}{lll}111.6413991 & 0.886721813 & 0.94 \\ 110.6620886 & 0.902485448 & 0.94\end{array}$ $\begin{array}{llll}110.098718 & 0.91174504 & 0.94\end{array}$ $\begin{array}{llll}108.9106023 & 0.931746164 & 0.94\end{array}$ $\begin{array}{lll}108.9106023 & 0.931746164 & 0.94 \\ 108.6760497 & 0.93577243 & 0.94\end{array}$ $\begin{array}{llll}108.1326694 & 0.945200808 & 0.94\end{array}$ $\begin{array}{llll}108.1326694 & 0.945200808 & 0.94\end{array}$ $\begin{array}{llll}108.1326694 & 0.945200808 & 0.94\end{array}$ $\begin{array}{llll}106.0867114 & 0.982010111 & 0.94\end{array}$ $\begin{array}{lll}106.012421 & 0.983386921 & 0.94\end{array}$ $\begin{array}{lll}105.5688544 & 0.991668037 & 0.94\end{array}$ $\begin{array}{lll}105.3484601 & 0.995821616 & 0.94 \\ 11048377681 & 1.005547057 & 0.94\end{array}$ $\begin{array}{llll}104.8377681 & 1.005547057 & 0.94\end{array}$ $\begin{array}{llll}0.83788127 & 1.00547057 & 0.94\end{array}$ $\begin{array}{lll}104.62644865 & 1.036420402 & 0.94\end{array}$ $\begin{array}{llll}101.3970108 & 1.074948427 & 0.94\end{array}$ $\begin{array}{lll}01.1260769 & 1.080716081 & 0.94\end{array}$ $\begin{array}{llll}00.9238248 & 1.085051948 & 0.94\end{array}$ $\begin{array}{lll}100.5217378 & 1.093749725 & 0.94\end{array}$ $\begin{array}{lll}100.3218934 & 1.098111633 & 0.94\end{array}$ \begin{tabular}{llll}
93.16045366 & 1.273429023 & 0.94 \\
\hline
\end{tabular} $\begin{array}{llll}9.86778943 & 1.338498865 & 0.94\end{array}$ $\begin{array}{lll}114.2959934 & 0.85049667 & 0.94 \\ 110.3891486 & 0.91176279 & 0.94\end{array}$ $\begin{array}{llll}108.6507368 & 0.94117261 & 0.94\end{array}$ $\begin{array}{llll}108.4179138 & 0.945219209 & 0.94\end{array}$ $\begin{array}{llll}08.6489924 & 0.958770566 & 0.94\end{array}$ $\begin{array}{llll}107.1928526 & 0.966947677 & 0.94\end{array}$ $\begin{array}{llll}0.7735744 & 0.993070932 & 0.94\end{array}$ $\begin{array}{llll}05.406305 & 1.000003343 & 0.94\end{array}$ $\begin{array}{llll}03.536889 & 1.036440579 & 0.94\end{array}$ $\begin{array}{lll}102.9051385 & 1.049205379 & 0.94\end{array}$ $\begin{array}{lll}101.800858 & 1.072091258 & 0.94\end{array}$ $\begin{array}{llll}100.5865336 & 1.098133011 & 0.94\end{array}$ $\begin{array}{llll}98.94724855 & 1.134830538 & 0.94\end{array}$ $\begin{array}{llll}85.94851601 & 1.504034735 & 0.94\end{array}$ $\begin{array}{llll}130.7766016 & 0.855490533 & 0.94\end{array}$ $\begin{array}{llll}119.0585887 & 1.032176203 & 0.94\end{array}$ $\begin{array}{llll}135.2629214 & 0.803621488 & 0.94\end{array}$ $\begin{array}{llll}129.5391007 & 0.876208196 & 0.94\end{array}$ $\begin{array}{llll}133.5230084 & 0.82875815 & 0.94\end{array}$ $\begin{array}{llll}32.3109993 & 0.844011047 & 0.94\end{array}$ $\begin{array}{llll}32.0114272 & 0.847846004 & 0.94\end{array}$ $\begin{array}{llll}131.7132086 & 0.851689653 & 0.94\end{array}$ $\begin{array}{llll}13.3667711 & 0.88014024 & 0.94\end{array}$ $\begin{array}{lll}128.9934668 & 0.887982895 & 0.94\end{array}$ $\begin{array}{llll}128.6140743 & 0.893229459 & 0.94\end{array}$ $\begin{array}{llll}25.838231 & 0.933071279 & 0.94\end{array}$ $\begin{array}{llll}123.0064283 & 0.976527356 & 0.94\end{array}$ $\begin{array}{lll}122.232803 & 0.988927579 & 0.94\end{array}$ $\begin{array}{llll}113.5075542 & 1.146807431 & 0.94\end{array}$ $\begin{array}{llll}104.3024097 & 1.358161284 & 0.94\end{array}$ $\begin{array}{lll}141.6329348 & 0.740183993 & 0.94 \\ 133.2382776 & 0.836392593 & 0.94\end{array}$ 


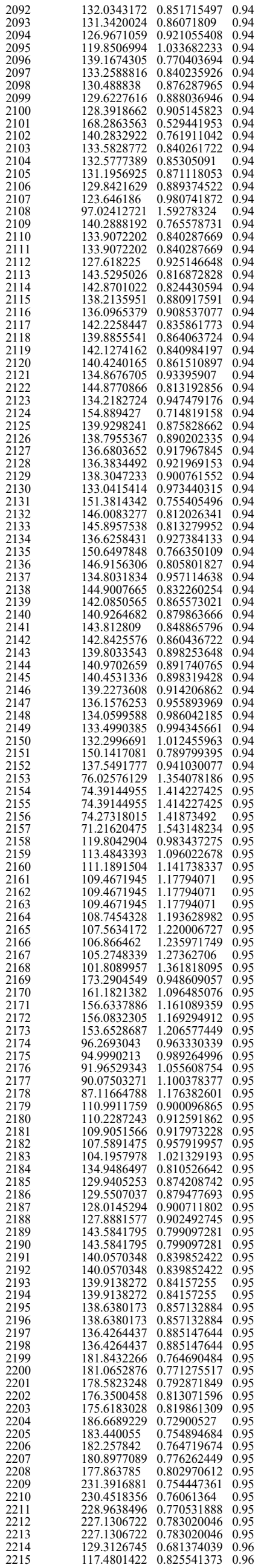




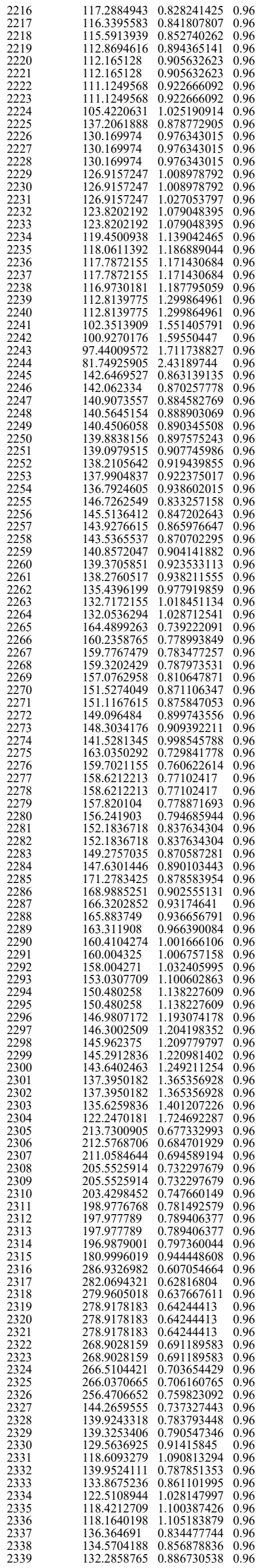




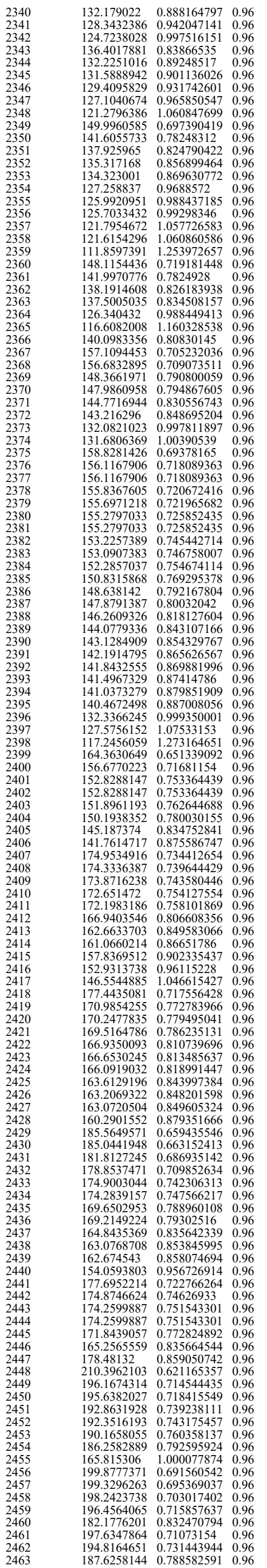




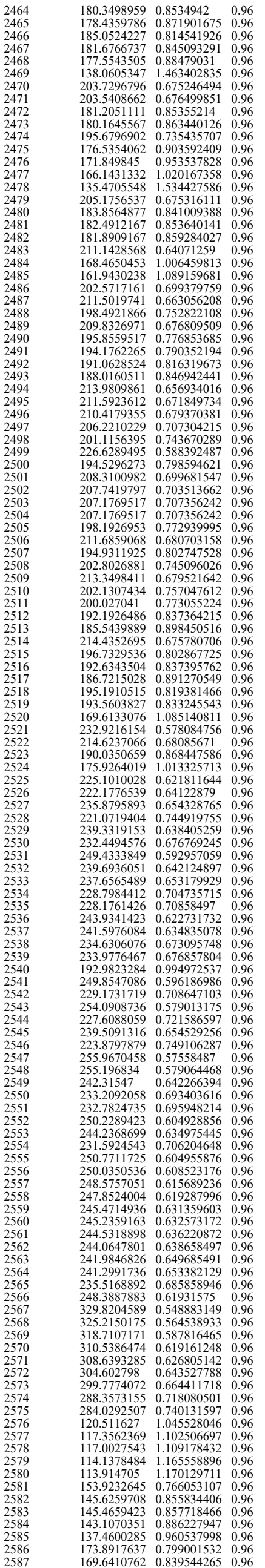




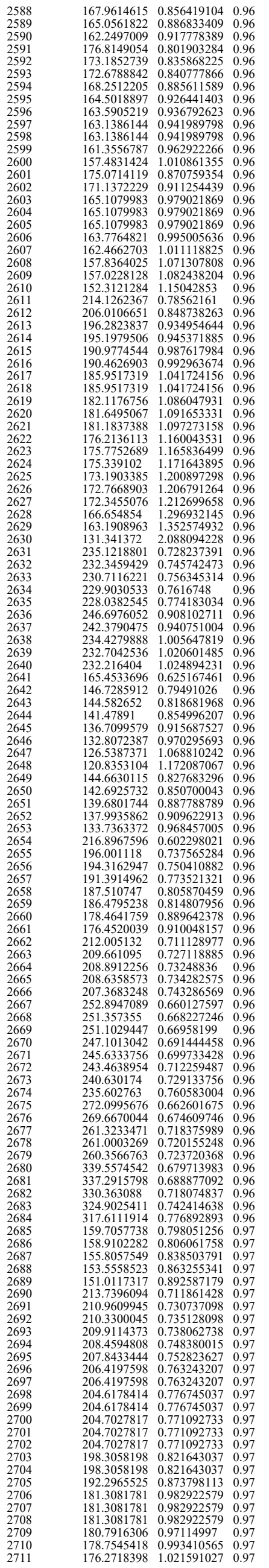




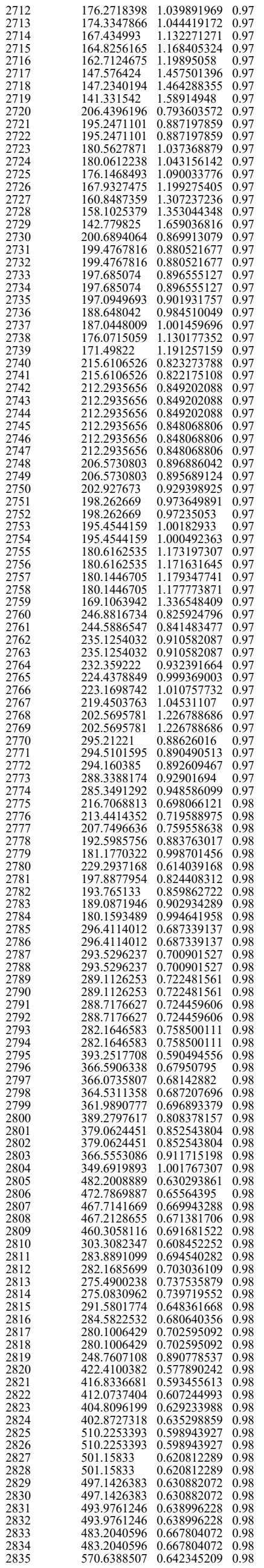




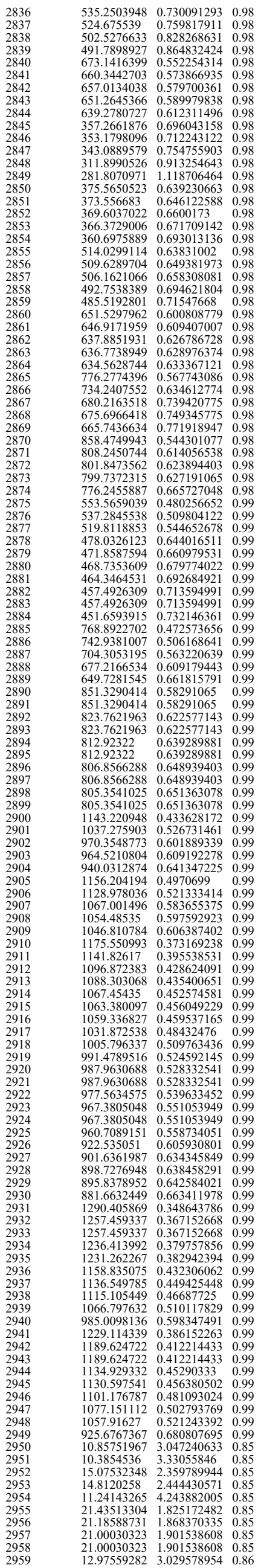



$\begin{array}{llll}25.79195084 & 1.755412421 & 0.86\end{array}$ $\begin{array}{llll}25.74268254 & 1.762138133 & 0.86\end{array}$ $\begin{array}{llll}22.69287815 & 2.288748132 & 0.86\end{array}$ $\begin{array}{llll}22.32488553 & 2.364823234 & 0.86\end{array}$ $\begin{array}{llll}21.73738854 & 2.494378926 & 0.86\end{array}$ $\begin{array}{lll}21.1800196 & 2.627389437 & 0.86\end{array}$ $\begin{array}{llll}21.07195828 & 2.654406117 & 0.86 \\ 20.75429057 & 2.73628515 & 0.86\end{array}$ $\begin{array}{llll}20.75429057 & 2.736285315 & 0.86 \\ 2.24560697 & 2.875514498 & 0.86\end{array}$ $\begin{array}{llll}20.24560697 & 2.875514498 & 0.86\end{array}$ $\begin{array}{llll}20.4090672 & 1.834321339 & 0.85 \\ 19.93149135 & 1.923266931 & 0.85\end{array}$ $\begin{array}{llll}26.32053253 & 1.619287004 & 0.85\end{array}$ $\begin{array}{llll}26.32053253 & 1.619287004 & 0.85 \\ 25.3629517 & 1.743867905 & 0.85 \\ 24.5658303 & 1.85885387 & 0.85\end{array}$ $\begin{array}{llll}24.56583036 & 1.858875387 & 0.85\end{array}$ $\begin{array}{lll}38.56763836 & 1.230202302 & 0.85\end{array}$ $\begin{array}{llll}38.27596919 & 1.249022423 & 0.85\end{array}$ $\begin{array}{llll}37.98867843 & 1.267985412 & 0.85\end{array}$ $\begin{array}{llll}23.99584997 & 2.10589567 & 0.85 \\ 23.95101886 & 2.113786607 & 0.85\end{array}$ $\begin{array}{llll}23.95101886 & 2.113786607 & 0.85\end{array}$ $\begin{array}{llll}35.67831088 & 1.35906713 & 0.85 \\ 33.84410253 & 1.510370434 & 0.85\end{array}$ $\begin{array}{llll}31.84046657 & 1.710282333 & 0.85\end{array}$ $\begin{array}{llll}31.59132171 & 1.73346025 & 0.85\end{array}$ $\begin{array}{llll}31.5132171 & 1.73346025 & 0.85 \\ 36.25339733 & 1.418870487 & 0.85\end{array}$ $\begin{array}{llll}34.3034623 & 1.584762787 & 0.85\end{array}$ $\begin{array}{llll}34.08635178 & 1.605015141 & 0.85\end{array}$ $\begin{array}{llll}32.61829895 & 1.752740249 & 0.85\end{array}$ $\begin{array}{llll}32.5963633 & 1.755100047 & 0.8\end{array}$ $\begin{array}{llll}32.24936276 & 1.793072715 & 0.85\end{array}$ $\begin{array}{llll}31.8886791 & 1.833863965 & 0.85\end{array}$ $\begin{array}{llll}31.82586489 & 1.841110045 & 0.85 \\ 31.61825977 & 1.865366833 & 0.85\end{array}$ $\begin{array}{llll}31.61825977 & 1.865366833 & 0.85 \\ 31.09095076 & 1.929177425 & 0.85\end{array}$ $\begin{array}{llll}31.09095076 & 1.929177425 & 0.85\end{array}$ $\begin{array}{lllll}30.10608213 & 2.057461385 & 0.85\end{array}$ $\begin{array}{lllll}30.08739431 & 2.060018031 & 0.85\end{array}$ $\begin{array}{llll}28.95507302 & 2.224287126 & 0.85\end{array}$ $\begin{array}{llll}28.19708681 & 2.345479785 & 0.85\end{array}$ $\begin{array}{llll}28.09900999 & 2.361881692 & 0.85\end{array}$ $\begin{array}{llll}28.03400361 & 2.372848046 & 0.85\end{array}$ $\begin{array}{llll}35.51679869 & 1.74005051 & 0.85\end{array}$ $\begin{array}{llll}35.27843091 & 1.763644146 & 0.85 \\ 34.58214609 & 1.835378332 & 0.85\end{array}$ $\begin{array}{llll}34.58214609 & 1.835378332 & 0.85 \\ 34.35611899 & 1.859607487 & 0.85\end{array}$ $\begin{array}{llll}34.35611899 & 1.859607487 & 0.85 \\ 34.13302731 & 1.883995522 & 0.85\end{array}$ $\begin{array}{llll}34.79712836 & 1.626738477 & 0.85\end{array}$ $\begin{array}{lllll}40.23440935 & 1.672559855 & 0.85\end{array}$ $\begin{array}{llll}39.15429098 & 1.766111835 & 0.85\end{array}$ $\begin{array}{lllll}38.89326237 & 1.789897585 & 0.85\end{array}$ $\begin{array}{llll}38.6356911 & 1.813842438 & 0.85\end{array}$ $\begin{array}{llll}38.38150892 & 1.837946392 & 0.85\end{array}$ $\begin{array}{llll}37.63864101 & 1.911212866 & 0.85\end{array}$ $\begin{array}{llll}37.63864101 & 1.911212866 & 0.85 \\ 37.39736767 & 1.912953228 & 0.85\end{array}$ $\begin{array}{llll}37.39736767 & 1.935953228 & 0.85\end{array}$ $\begin{array}{llll}36.49743347 & 2.036505697 & 0.85\end{array}$ $\begin{array}{lllll}48.42152376 & 1.231499682 & 0.85\end{array}$ $\begin{array}{llll}4.46973606 & 1.231439589 & 0.85\end{array}$ $\begin{array}{llll}46.37434249 & 1.342627932 & 0.85\end{array}$ $\begin{array}{llll}45.86266275 & 1.372753869 & 0.85\end{array}$ $\begin{array}{llll}45.0259437 & 1.424247809 & 0.85\end{array}$ $\begin{array}{llll}33.6811347 & 1.470109397 & 0.90\end{array}$ $\begin{array}{llll}31.86609577 & 1.642348688 & 0.90\end{array}$ $\begin{array}{llll}31.32658241 & 1.699405605 & 0.90 \\ 31.2261117 & 1.721753712 & 0.90\end{array}$ $\begin{array}{llll}31.12261117 & 1.721753712 & 0.90\end{array}$ $\begin{array}{llll}28.72257005 & 2.021512762 & 0.90\end{array}$ $\begin{array}{llll}42.15045141 & 1.3812116 & 0.90\end{array}$ $\begin{array}{lll}41.97238674 & 1.39295583 & 0.90\end{array}$ $\begin{array}{llll}41.69559042 & 1.411511501 & 0.90\end{array}$ $\begin{array}{llll}38.89722865 & 1.621912263 & 0.90\end{array}$ $\begin{array}{lll}48.11351964 & 1.28314441 & 0.90\end{array}$ $\begin{array}{llll}46.70874534 & 1.361486654 & 0.90\end{array}$ $\begin{array}{llll}46.40764142 & 1.379211274 & 0.90\end{array}$ $\begin{array}{llll}45.74414853 & 1.419510782 & 0.90\end{array}$ $\begin{array}{llll}45.67159636 & 1.424024327 & 0.90\end{array}$ $\begin{array}{llll}45.67159636 & 1.424024327 & 0.90\end{array}$ $\begin{array}{llll}4.33555274 & 1.4421142909 & 0.90\end{array}$ $\begin{array}{llll}43.72959985 & 1.553312315 & 0.90\end{array}$ $\begin{array}{llll}43.53127967 & 1.567497742 & 0.90\end{array}$ $\begin{array}{llll}43.40006255 & 1.576990515 & 0.90\end{array}$ $\begin{array}{llll}43.26963411 & 1.586511945 & 0.90\end{array}$ $\begin{array}{llll}42.0685778 & 1.678394825 & 0.90\end{array}$ $\begin{array}{llll}41.6427209 & 1.712898368 & 0.90\end{array}$ $\begin{array}{llll}41.6427209 & 1.712898368 & 0.90\end{array}$ $\begin{array}{llll}40.41535649 & 1.81851532 & 0.90\end{array}$ $\begin{array}{llll}38.91343446 & 1.961600964 & 0.90\end{array}$ $\begin{array}{llll}0.7753075 & 1.356008712 & 0.90\end{array}$ $\begin{array}{llll}49.96509025 & 1.300465075 & 0.90\end{array}$ $\begin{array}{llll}49.17823844 & 1.445638476 & 0.90\end{array}$ $\begin{array}{lllll}48.41578513 & 1.491528916 & 0.90\end{array}$ $\begin{array}{llll}48.04335601 & 1.514743025 & 0.90\end{array}$ $\begin{array}{llll}46.60922598 & 1.609392057 & 0.90\end{array}$ $\begin{array}{lll}46.26397246 & 1.633502463 & 0.90\end{array}$ $\begin{array}{llll}55.90171187 & 1.380110718 & 0.90\end{array}$ $\begin{array}{llll}55.45449817 & 1.402460326 & 0.90\end{array}$ $\begin{array}{llll}55.01438311 & 1.424989449 & 0.90\end{array}$ $\begin{array}{llll}54.58119899 & 1.447698086 & 0.90\end{array}$ $\begin{array}{lllll}51.72994233 & 1.411684967 & 0.90\end{array}$ $\begin{array}{llll}59.86044633 & 1.330124402 & 0.90\end{array}$ $\begin{array}{llll}59.74707427 & 1.335177101 & 0.90\end{array}$ $\begin{array}{llll}59.37224946 & 1.352088613 & 0.90\end{array}$ $\begin{array}{llll}59.37224946 & 1.352088613 & 0.90\end{array}$ $\begin{array}{lll}58.02536214 & 1.4155866 & 0.90\end{array}$ $\begin{array}{llll}41.61859192 & 1.342809631 & 0.87\end{array}$ $\begin{array}{llll}41.30008228 & 1.363601213 & 0.87\end{array}$ $\begin{array}{lll}38.96421722 & 1.531994835 & 0.87\end{array}$ 


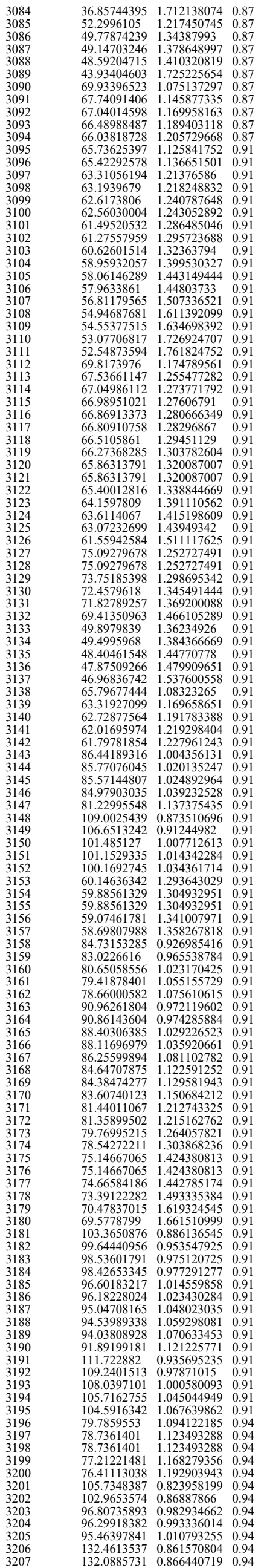


$\begin{array}{llll}128.7078784 & 0.912555083 & 0.94\end{array}$ $\begin{array}{llll}128.3558969 & 0.917566818 & 0.94 \\ 118.8239822 & 1.070683587 & 0.94\end{array}$ $\begin{array}{llll}118.8239822 & 1.070683587 & 0.94 \\ 124.9247223 & 0.851491393 & 0.94\end{array}$ $\begin{array}{llll}12.763944 & 0.853687373 & 0.94\end{array}$ $\begin{array}{lll}123.1786334 & 0.87580271 & 0.94\end{array}$ $\begin{array}{lll}121.1769806 & 0.904975442 & 0.94\end{array}$ $\begin{array}{llll}119.9772085 & 0.923165448 & 0.94\end{array}$ $\begin{array}{llll}119.0928556 & 0.936926731 & 0.94\end{array}$ $\begin{array}{llll}118.365793 & 0.948472238 & 0.94 \\ 117.0792083 & 0.969432318 & 0.94\end{array}$ $\begin{array}{lllll}117.0792083 & 0.969432318 & 0.94\end{array}$ $\begin{array}{llll}116.5163275 & 0.978821438 & 0.94\end{array}$ $\begin{array}{llll}116.5163275 & 0.978821438 & 0.94 \\ 116.369118 & 0.983532966 & 0.94\end{array}$ $\begin{array}{llll}115.2694227 & 1.000112399 & 0.94\end{array}$ $\begin{array}{llll}114.9959484 & 1.004874832 & 0.94\end{array}$ $\begin{array}{llll}113.1173681 & 1.038528607 & 0.94\end{array}$ $\begin{array}{llll}110.2862167 & 1.092533016 & 0.94\end{array}$ $\begin{array}{llll}101.0861152 & 1.300451124 & 0.94\end{array}$ $\begin{array}{llll}99.63163871 & 1.338697648 & 0.94\end{array}$ $\begin{array}{llll}142.6454873 & 0.768715043 & 0.94\end{array}$ $\begin{array}{lllll}142.0671948 & 0.774985976 & 0.94\end{array}$ $\begin{array}{llll}13.800165 & 0.800324451 & 0.94\end{array}$ $\begin{array}{llll}136.1783991 & 0.843460997 & 0.94\end{array}$ $\begin{array}{llll}134.9547165 & 0.858826269 & 0.94\end{array}$ $\begin{array}{llll}133.9232154 & 0.87210689 & 0.94\end{array}$ $\begin{array}{llll}132.7395507 & 0.887729736 & 0.94\end{array}$ $\begin{array}{lll}130.2722728 & 0.921674291 & 0.94\end{array}$ $\begin{array}{llll}128.2069806 & 0.951608054 & 0.94\end{array}$ $\begin{array}{llll}147.6959331 & 0.884539794 & 0.94\end{array}$ $\begin{array}{llll}144.0491199 & 0.929893541 & 0.94\end{array}$ $\begin{array}{llll}144.0491199 & 0.929893541 & 0.94\end{array}$ $\begin{array}{llll}144.0491199 & 0.92989541 & 0.94 \\ 142.2924233 & 0.952995606 & 0.94\end{array}$ $\begin{array}{llll}98.79993071 & 0.946568951 & 0.95\end{array}$ $\begin{array}{llll}94.26047443 & 1.039935232 & 0.95\end{array}$ $\begin{array}{llll}93.25234102 & 1.062541859 & 0.95\end{array}$ $\begin{array}{llll}93.00366811 & 1.068231498 & 0.95\end{array}$ $\begin{array}{lll}90.5096251 & 1.12791404 & 0.95\end{array}$ $\begin{array}{llll}127.1893646 & 0.786561145 & 0.95\end{array}$ $\begin{array}{lll}117.6776 & 0.918853935 & 0.95\end{array}$ $\begin{array}{llll}114.8154694 & 0.96523547 & 0.95 \\ 113.540922 & 0.987027494 & 0.95\end{array}$ $\begin{array}{llll}113.5409221 & 0.987027494 & 0.95 \\ 110.8741977 & 1.035078063 & 0.95\end{array}$

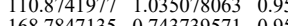
$\begin{array}{llll}163.2108117 & 0.79540673 \quad 0.95\end{array}$ $\begin{array}{llll}158.21512766 & 0.847113703 & 0.95\end{array}$ $\begin{array}{llll}154.2939283 & 0.889998834 & 0.95\end{array}$ $\begin{array}{llll}151.9224559 & 0.918001025 & 0.95\end{array}$ $\begin{array}{llll}122.375684 & 0.867451084 & 0.96\end{array}$ $\begin{array}{llll}120.7440082 & 0.891054118 & 0.96\end{array}$ $\begin{array}{llll}119.2759959 & 0.913122734 & 0.96\end{array}$ $\begin{array}{lll}118.0796469 & 0.931719461 & 0.96\end{array}$ $\begin{array}{llll}116.67533 & 0.95428299 & 0.96\end{array}$ $\begin{array}{llll}193.1853267 & 0.751682152 & 0.96\end{array}$ $\begin{array}{lll}193.5358774 & 0.756735463 & 0.96\end{array}$ $\begin{array}{lllll}187.6774941 & 0.804716055 & 0.96\end{array}$ $\begin{array}{llll}185.0764201 & 0.827494046 & 0.96\end{array}$ $\begin{array}{lll}127.250732 & 0.799472224 & 0.96\end{array}$ $\begin{array}{lllll}126.4190279 & 0.810026198 & 0.96\end{array}$ $\begin{array}{lll}126.0072396 & 0.815329138 & 0.96\end{array}$ $\begin{array}{llll}123.8555684 & 0.843903688 & 0.96\end{array}$ $\begin{array}{llll}123.8555684 & 0.843903688 & 0.96\end{array}$ $\begin{array}{llll}166.2589056 & 0.780969227 & 0.96\end{array}$ $\begin{array}{llll}163.4046755 & 0.808490272 & 0.96\end{array}$ $\begin{array}{llll}159.0805963 & 0.853039881 & 0.96\end{array}$ $\begin{array}{lllll}155.6128994 & 0.891481976 & 0.96\end{array}$ $\begin{array}{llll}155.6128994 & 0.891481976 & 0.96\end{array}$ $\begin{array}{llll}154.3511732 & 0.906116183 & 0.96\end{array}$ $\begin{array}{llll}153.7279518 & 0.913477963 & 0.96\end{array}$ $\begin{array}{llll}153.7279518 & 0.913477963 & 0.96\end{array}$ $\begin{array}{llll}151.6864119 & 0.938232342 & 0.96\end{array}$ $\begin{array}{lll}151.2845936 & 0.943222931 & 0.96\end{array}$ $\begin{array}{lll}150.091811 & 0.958274123 & 0.96\end{array}$ $\begin{array}{llll}149.5024453 & 0.965844396 & 0.96\end{array}$ $\begin{array}{llll}147.7617958 & 0.988733923 & 0.96 \\ 147.5708891 & 0.991293751 & 0.96\end{array}$ $\begin{array}{llll}142.9535271 & 1.056365006 & 0.96\end{array}$ $\begin{array}{llll}141.1864872 & 1.082972654 & 0.96\end{array}$ $\begin{array}{lll}39.8040002 & 1.10449705 \quad 0.96\end{array}$ $\begin{array}{llll}192.0425 & 0.688992363 & 0.96\end{array}$ $\begin{array}{llll}189.980694 & 0.704028387 & 0.96\end{array}$ $\begin{array}{llll}89.6897588 & 0.706189639 & 0.96\end{array}$ $\begin{array}{llll}179.7785377 & 0.786200694 & 0.96\end{array}$ $\begin{array}{llll}178.999151 & 0.793062049 & 0.96\end{array}$ $\begin{array}{llll}174.9539724 & 0.830159409 & 0.96\end{array}$ $\begin{array}{llll}174.7072108 & 0.832506148 & 0.96\end{array}$ $\begin{array}{llll}172.7578975 & 0.851399303 & 0.96\end{array}$ $\begin{array}{lll}21.324222 & 0.8659136106 & 0.96\end{array}$ $\begin{array}{lllll}207.9328223 & 0.701741415 & 0.96\end{array}$ $\begin{array}{llll}207.2040857 & 0.706686143 & 0.96\end{array}$ $\begin{array}{lll}202.704797 & 0.73840589 & 0.96\end{array}$ $\begin{array}{lll}199.7372718 & 0.760510085 & 0.96\end{array}$ $\begin{array}{llll}199.2407088 & 0.789637115 & 0.96\end{array}$ $\begin{array}{llll}199.2407088 & 0.789637115 & 0.96\end{array}$ $\begin{array}{llll}193.6282945 & 0.836076601 & 0.96\end{array}$ $\begin{array}{llll}190.9390126 & 0.85979391 & 0.96\end{array}$ $\begin{array}{llll}185.778498 & 0.959223659 & 0.96\end{array}$ $\begin{array}{llll}185.7784988 & 0.908223659 & 0.96\end{array}$ $\begin{array}{llll}83.3014521 & 0.9329361 & 0.96\end{array}$ $\begin{array}{llll}178.5403754 & 0.983356113 & 0.96\end{array}$ $\begin{array}{llll}176.2513963 & 1.009063686 & 0.96\end{array}$ $\begin{array}{llll}174.0203659 & 1.035102969 & 0.96\end{array}$ $\begin{array}{llll}174.0203659 & 1.035102969 & 0.96\end{array}$ $\begin{array}{llll}156.9816919 & 0.835417824 & 0.96\end{array}$ $\begin{array}{llll}153.8018922 & 0.870318852 & 0.96\end{array}$ $\begin{array}{llll}150.2772655 & 0.911622804 & 0.96\end{array}$ $\begin{array}{llll}142.9793606 & 1.007059305 & 0.96\end{array}$ $\begin{array}{llll}146.6636056 & 0.846047858 & 0.96\end{array}$ $\begin{array}{llll}145.6923235 & 0.857366099 & 0.96\end{array}$ $145.0519177 \quad 0.864953372 \quad 0.96$ $\begin{array}{llll}144.5911327 & 0.870475046 & 0.96\end{array}$ $\begin{array}{llll}138.9444685 & 0.942664435 & 0.96\end{array}$ $\begin{array}{llll}181.0625238 & 0.739252307 & 0.96\end{array}$ $\begin{array}{llll}170.9018883 & 0.829766897 & 0.96\end{array}$ $\begin{array}{llll}170.328392 & 0.83536396 & 0.96\end{array}$ $\begin{array}{llll}174.6200892 & 0.894301918 & 0.96\end{array}$ 
$\begin{array}{llll}239.2893152 & 0.692739291 & 0.96\end{array}$ $\begin{array}{llll}236.665592 & 0.708161586 & 0.96\end{array}$ $\begin{array}{llll}234.9543639 & 0.718537441 & 0.96\end{array}$ $\begin{array}{llll}224.6445881 & 0.786003561 & 0.96\end{array}$ $\begin{array}{llll}179.4288791 & 0.674925401 & 0.97\end{array}$ $\begin{array}{llll}177.3611967 & 0.690753732 & 0.97\end{array}$ $\begin{array}{llll}173.1488683 & 0.724771565 & 0.97\end{array}$ $\begin{array}{llll}172.5019858 & 0.730217544 & 0.97\end{array}$ $\begin{array}{llll}175.297249 & 0.795260129 & 0.97\end{array}$ $\begin{array}{llll}213.1304385 & 0.67539934 & 0.97\end{array}$ $\begin{array}{llll}198.476114 & 0.778816407 & 0.97\end{array}$ $\begin{array}{llll}19.476114 & 0.778816407 & 0.97 \\ 191.3217425 & 0.83815286 & 0.97\end{array}$ $\begin{array}{llll}242.6333404 & 0.604831128 & 0.97\end{array}$ $\begin{array}{llll}238.7752131 & 0.624534747 & 0.97\end{array}$ $\begin{array}{llll}237.5162928 & 0.631172802 & 0.97\end{array}$ $\begin{array}{llll}233.4141116 & 0.653553088 & 0.97\end{array}$ $\begin{array}{llll}233.0116734 & 0.655812561 & 0.97\end{array}$ $\begin{array}{llll}227.9034917 & 0.685540522 & 0.97\end{array}$ $\begin{array}{llll}227.5198158 & 0.687854581 & 0.97\end{array}$ $\begin{array}{llll}227.1374296 & 0.690172539 & 0.97\end{array}$ $\begin{array}{llll}226.753265 & 0.692494396 & 0.97\end{array}$ $\begin{array}{llll}221.9158795 & 0.723033349 & 0.97\end{array}$ $\begin{array}{llll}221.9158759 & 0.723033449 & 0.97 \\ 220.1087469 & 0.734954555 & 0.97\end{array}$ $\begin{array}{llll}215.5450887 & 0.766405875 & 0.97\end{array}$ $\begin{array}{lllll}213.5020072 & 0.781144113 & 0.97\end{array}$ $\begin{array}{lll}213.1652533 & 0.783614132 & 0.97\end{array}$ $\begin{array}{lll}212.82956 & 0.78608805 & 0.97\end{array}$ $\begin{array}{llll}194.455785 & 0.941658331 & 0.97\end{array}$ $\begin{array}{llll}252.6929292 & 0.656379276 & 0.97\end{array}$ $\begin{array}{llll}248.4099982 & 0.679208162 & 0.97\end{array}$ $\begin{array}{llll}244.2698316 & 0.702427287 & 0.97\end{array}$ $\begin{array}{llll}244.2698316 & 0.702427287 & 0.97\end{array}$ $\begin{array}{llll}244.2638316 & 0.702427287 & 0.97\end{array}$ $\begin{array}{lll}282.9008513 & 0.634120629 & 0.97\end{array}$ $\begin{array}{llll}276.9684612 & 0.661576018 & 0.97\end{array}$ $\begin{array}{llll}272.6799173 & 0.68254939 & 0.97\end{array}$ $\begin{array}{lll}270.5850652 & 0.693158807 & 0.97\end{array}$ $\begin{array}{llll}270.5850652 & 0.693158807 & 0.97\end{array}$ $\begin{array}{llll}280.4550039 & 0.657340307 & 0.97\end{array}$ $\begin{array}{lllll}280.450039 & 0.657340307 & 0.97\end{array}$ $\begin{array}{llll}280.455039 & 0.657340307 & 0.97\end{array}$ $\begin{array}{lllll}266.6621349 & 0.727099668 & 0.97\end{array}$ $\begin{array}{llll}266.6621349 & 0.727099688 & 0.97 \\ 175.5618501 & 0.827918803 & 0.97\end{array}$ $\begin{array}{llll}172.2917807 & 0.85964457 & 0.97\end{array}$ $\begin{array}{llll}170.8991219 & 0.873712161 & 0.97\end{array}$ $\begin{array}{lll}167.4192756 & 0.910410225 & 0.97\end{array}$ $\begin{array}{llll}164.4429329 & 0.943664496 & 0.97\end{array}$ $\begin{array}{llll}214.6606821 & 0.729399604 & 0.97\end{array}$ $\begin{array}{llll}213.312311 & 0.738649982 & 0.97\end{array}$ $\begin{array}{llll}209.8845483 & 0.762973755 & 0.97\end{array}$ $\begin{array}{lllll}202.138809 & 0.822566708 & 0.97\end{array}$ $\begin{array}{lllll}196.296469 & 0.872257618 & 0.97\end{array}$ $\begin{array}{llll}269.1030807 & 0.77082053 & 0.97\end{array}$ $\begin{array}{llll}267.783948 & 0.778433526 & 0.97\end{array}$ $\begin{array}{lllll}265.8293206 & 0.789923164 & 0.97\end{array}$ $\begin{array}{llll}265.5063202 & 0.791846287 & 0.97\end{array}$ $\begin{array}{lll}247.452303 & 0.60485883 & 0.98\end{array}$ $\begin{array}{llll}243.4435028 & 0.624943347 & 0.98\end{array}$ $\begin{array}{llll}242.728544 & 0.62863032 & 0.98\end{array}$ $\begin{array}{llll}240.2589221 & 0.641620124 & 0.98\end{array}$ $\begin{array}{llll}237.1565831 & 0.658516493 & 0.98\end{array}$ $\begin{array}{llll}2877.7265859 & 0.52954645 & 0.98\end{array}$ $\begin{array}{llll}275.0857625 & 0.539762545 & 0.98\end{array}$ $\begin{array}{llll}270.794253 & 0.557006272 & 0.98\end{array}$ $\begin{array}{llll}265.4114926 & 0.579828453 & 0.98\end{array}$ $\begin{array}{llll}303.0254084 & 0.573097753 & 0.98\end{array}$ $\begin{array}{llll}293.1049338 & 0.612548582 & 0.98\end{array}$ $\begin{array}{llll}281.3807364 & 0.664657749 & 0.98\end{array}$ $\begin{array}{llll}278.9893987 & 0.676100714 & 0.98\end{array}$ $\begin{array}{lllll}278.5947885 & 0.67801737 & 0.98\end{array}$ $\begin{array}{lllll}371.1997649 & 0.445626101 & 0.98\end{array}$ $\begin{array}{llll}355.733108 & 0.48521859 & 0.98 \\ 327.705651 & 0.57176576 & 0.98\end{array}$ $\begin{array}{llll}319.3194828 & 0.502192262 \quad 0.98\end{array}$ $\begin{array}{llll}318.6921361 & 0.604565431 & 0.98\end{array}$ $\begin{array}{llll}312.5516325 & 0.628553808 & 0.98\end{array}$ $\begin{array}{llll}310.1611802 & 0.638279835 & 0.98\end{array}$ $\begin{array}{llll}306.6432841 & 0.653008885 & 0.98\end{array}$ $\begin{array}{llll}306.0647118 & 0.655480061 & 0.98\end{array}$ $\begin{array}{llll}302.0750415 & 0.67290897 & 0.98\end{array}$ $\begin{array}{llll}297.6409124 & 0.693107743 & 0.98\end{array}$ $\begin{array}{llll}296.5526458 & 0.698204107 & 0.98\end{array}$ $\begin{array}{llll}294.3998136 & 0.708452837 & 0.98\end{array}$ $\begin{array}{llll}288.6375396 & 0.737021874 & 0.98 \\ 283.0965048 & 0.766155617 & 0.98\end{array}$ $\begin{array}{lllll}279.1984462 & 0.787698487 & 0.98\end{array}$ $\begin{array}{llll}270.3571621 & 0.840059886 & 0.98\end{array}$ $\begin{array}{lllll}263.763085 & 0.882587918 & 0.98\end{array}$ $\begin{array}{llll}400.0411994 & 0.551983597 & 0.98\end{array}$ $\begin{array}{llll}398.1660063 & 0.557195057 & 0.98\end{array}$ $\begin{array}{llll}392.0403754 & 0.574743437 & 0.98\end{array}$ $\begin{array}{llll}380.9061943 & 0.608834905 & 0.98\end{array}$ $\begin{array}{llll}372.5529883 & 0.636442995 & 0.98\end{array}$ $\begin{array}{lllll}398.454342 & 0.561579802 & 0.98\end{array}$ $\begin{array}{lllll}382.82286815 & 0.608358627 & 0.98\end{array}$ $\begin{array}{llll}375.4665915 & 0.632449723 & 0.98\end{array}$ $\begin{array}{llll}368.3823162 & 0.657008606 & 0.98\end{array}$ $\begin{array}{lllll}368.3823162 & 0.657008606 & 0.98\end{array}$ $\begin{array}{llll}471.4600025 & 0.401124232 & 0.98\end{array}$ $\begin{array}{llll}470.5933481 & 0.402603029 & 0.98\end{array}$ $\begin{array}{llll}426.6713023 & 0.489758227 & 0.98\end{array}$ $\begin{array}{llll}426.6713023 & 0.489758227 & 0.98\end{array}$ $\begin{array}{llll}390.2481423 & 0.585446101 & 0.98\end{array}$ $\begin{array}{lllll}338.0727952 & 0.546031803 & 0.98\end{array}$ $\begin{array}{llll}332.3524433 & 0.564989836 & 0.98\end{array}$ $\begin{array}{lllll}327.3671567 & 0.582328672 & 0.98\end{array}$ $\begin{array}{llll}324.1258977 & 0.594033479 & 0.98\end{array}$ $\begin{array}{llll}402.045375 & 0.552061955 & 0.98\end{array}$ $\begin{array}{lllll}395.2769343 & 0.571130054 & 0.98\end{array}$ $\begin{array}{llll}387.4496683 & 0.594439107 & 0.98\end{array}$ $\begin{array}{llll}370.3383265 & 0.650639822 & 0.98\end{array}$ $\begin{array}{llll}368.59419 & 0.656811865 & 0.98\end{array}$ $\begin{array}{llll}464.9476473 & 0.4842257 & 0.98\end{array}$ $\begin{array}{lll}444.6861673 & 0.529357053 & 0.98 \\ 441.6473097 & 0.536666846 & 0.98\end{array}$ 


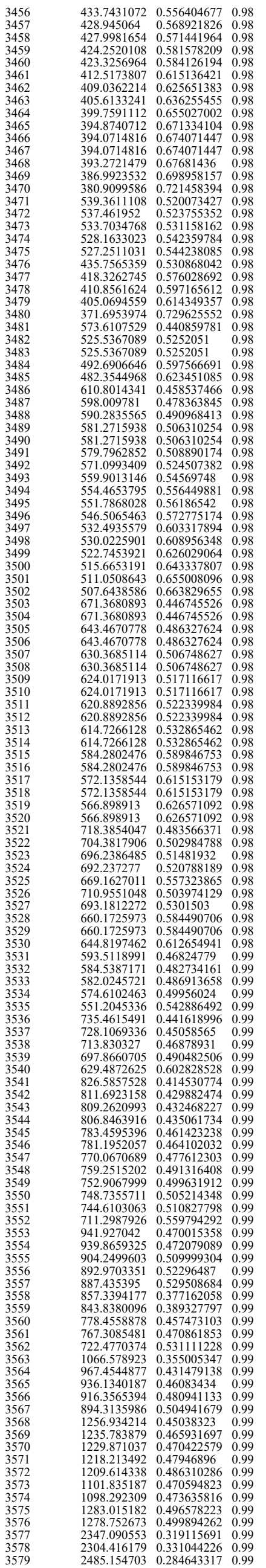




$\begin{array}{llll}3580 & 2369.025978 & 0.3132335 & 0.99 \\ 3581 & 2283.655673 & 0.337090571 & 0.99 \\ 3582 & 2166.545125 & 0.37451772 & 0.99 \\ 3583 & 2586.589589 & 0.262756095 & 0.99 \\ 3584 & 2204.224171 & 0.361823132 & 0.99 \\ 3585 & 2185.222239 & 0.368143067 & 0.99 \\ 3586 & 2094.923799 & 0.400563514 & 0.99 \\ 3587 & 2185.222239 & 0.368143067 & 0.99 \\ 3588 & 2204.224171 & 0.361823132 & 0.99 \\ 3589 & 2204.224171 & 0.361823132 & 0.99 \\ 3590 & 2185.222239 & 0.368143067 & 0.99 \\ 3591 & 2077.752292 & 0.407211757 & 0.99 \\ 3592 & 1949.890613 & 0.462367556 & 0.99 \\ 3593 & 1891.684923 & 0.491258688 & 0.99 \\ 3594 & 2027.886237 & 0.427484796 & 0.99 \\ 3595 & 2027.886237 & 0.427484796 & 0.99 \\ 3596 & 2112.381497 & 0.393969988 & 0.99 \\ 3597 & 1965.006044 & 0.455281568 & 0.99 \\ 3598 & 2011.791902 & 0.434351912 & 0.99 \\ 3599 & 1905.908118 & 0.483953828 & 0.99 \\ 3600 & 1656.769802 & 0.640447462 & 0.99 \\ 3601 & 1635.392127 & 0.657300623 & 0.99 \\ 3602 & 1760.317914 & 0.567316783 & 0.99 \\ 3603 & 1594.250187 & 0.691663561 & 0.99 \\ 3604 & 1736.20397 & 0.583185019 & 0.99 \\ 3605 & 1584.286123 & 0.70039109 & 0.99 \\ 3606 & 1810.612712 & 0.536236929 & 0.99 \\ 3607 & 1785.111125 & 0.55166742 & 0.99 \\ 3608 & 1810.612712 & 0.536236929 & 0.99 \\ 3609 & 1408.254332 & 0.886432474 & 0.99 \\ 3610 & 1408.254332 & 0.886432474 & 0.99 \\ 3611 & 1392.779009 & 0.906240409 & 0.99 \\ 3612 & 1.199722183 & 18.24376337 & 0.95 \\ 3613 & 4.093720973 & 6.522797222 & 0.95 \\ 3614 & 4.079919975 & 6.567000719 & 0.95 \\ 3615 & 4.076484253 & 6.578074917 & 0.95 \\ 3616 & 4.066211718 & 6.611353489 & 0.95 \\ 3617 & 4.063651669 & 6.619686252 & 0.95 \\ 3618 & 185.5476764 & 0.761340625 & 0.95 \\ 3619 & 399.2433031 & 0.684557743 & 0.95 \\ 3620 & 436.1103649 & 0.573710172 & 0.95 \\ 3621 & 430.2955601 & 0.589320621 & 0.95 \\ 3622 & 405.0899624 & 0.664939915 & 0.95 \\ 3623 & 400.068186 & 0.681737735 & 0.95 \\ 3624 & 634.5730534 & 0.520051712 & 0.95 \\ 3625 & 1489.484631 & 0.392946675 & 0.95 \\ 3626 & 1483.777793 & 0.395975155 & 0.95 \\ 3627 & 1483.777793 & 0.395975155 & 0.95 \\ 3628 & 1472.494312 & 0.402066991 & 0.95 \\ 3629 & 1455.887233 & 0.411291937 & 0.95 \\ & & & \end{array}$




\section{S17.3 SDC data (literature)}

Source: (Brown and Lawler, 2003)

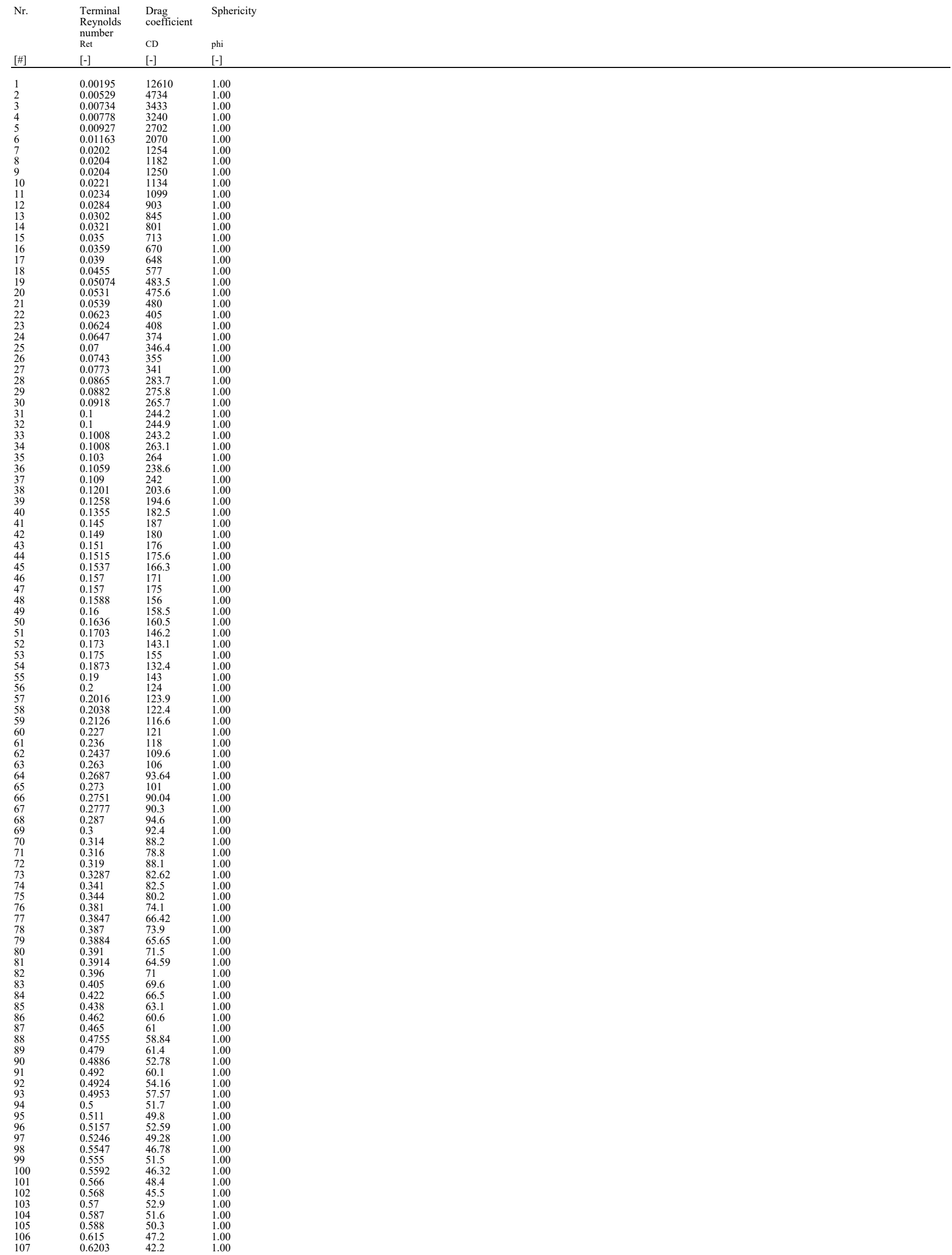




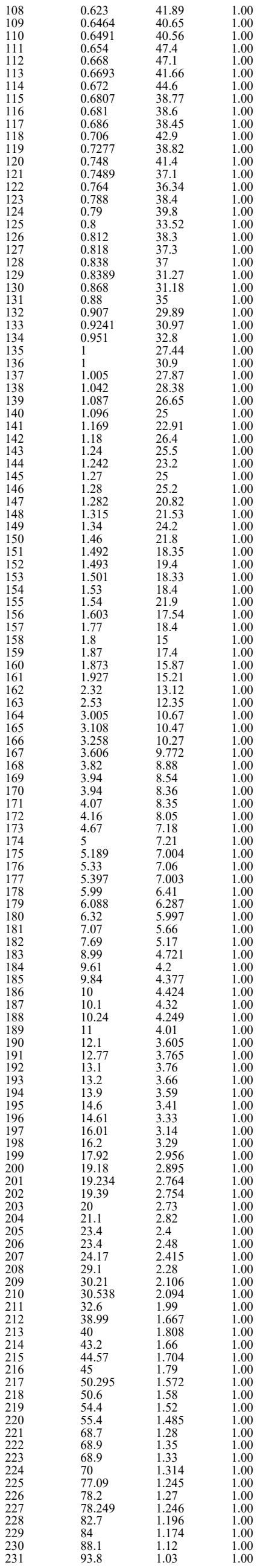




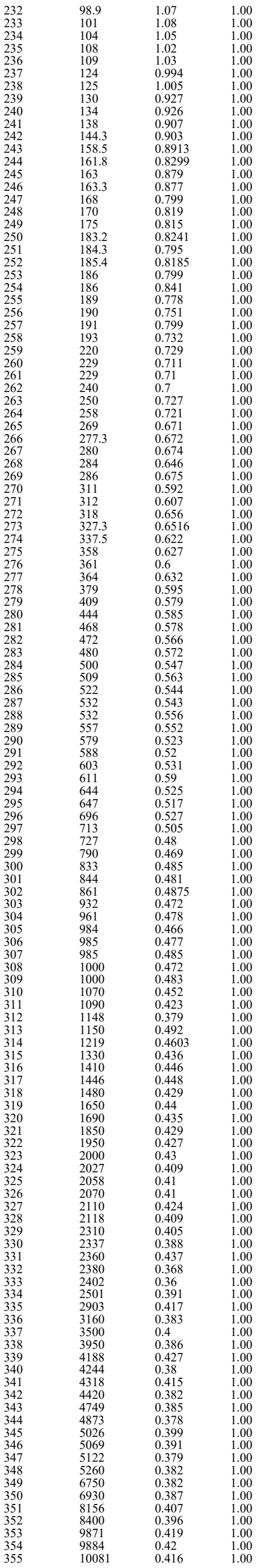




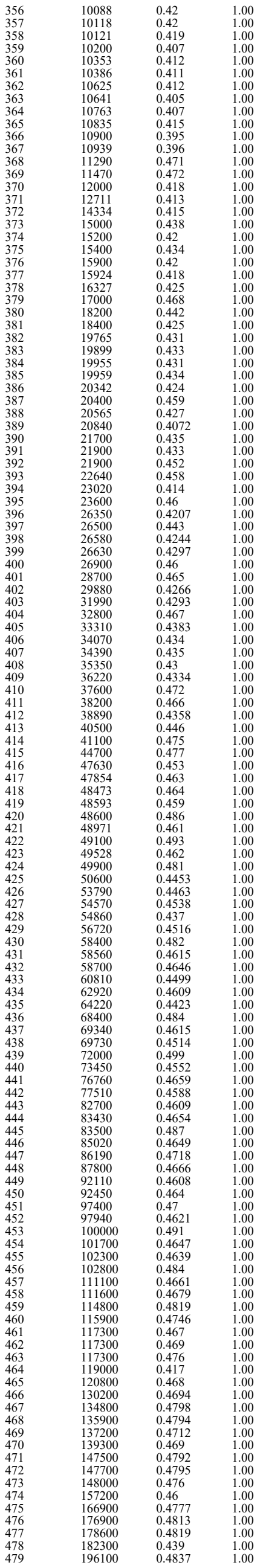


Source: (Almedeij, 2008)

\begin{tabular}{llll} 
Nr. & $\begin{array}{l}\text { Terminal } \\
\text { Reynolds }\end{array}$ & $\begin{array}{l}\text { Drag } \\
\text { coefficient }\end{array}$ & Spherici \\
& $\begin{array}{l}\text { Ret } \\
\text { Rer }\end{array}$ & CD & phi \\
[\#] & {$[-]$} & {$[-]$} & {$[-]$} \\
\hline & & & \\
1 & 0.1 & 240 & 1.00 \\
2 & 0.2 & 120 & 1.00 \\
3 & 0.3 & 80 & 1.00 \\
4 & 0.4 & 62 & 1.00 \\
5 & 0.8 & 33 & 1.00 \\
6 & 1 & 25 & 1.00 \\
7 & 4 & 8 & 1.00 \\
8 & 5.33 & 7.06 & 1.00 \\
9 & 5.99 & 6.41 & 1.00 \\
10 & 6 & 6.3 & 1.00 \\
11 & 10 & 4.2 & 1.00 \\
12 & 13.1 & 3.76 & 1.00 \\
13 & 14.6 & 3.41 & 1.00 \\
14 & 16.2 & 3.29 & 1.00 \\
15 & 21.1 & 2.82 & 1.00 \\
16 & 29.1 & 2.28 & 1.00 \\
17 & 45 & 1.79 & 1.00 \\
18 & 54.4 & 1.52 & 1.00 \\
19 & 78.2 & 1.27 & 1.00 \\
20 & 200 & 0.74 & 1.00 \\
21 & 400 & 0.58 & 1.00 \\
22 & 1000 & 0.46 & 1.00 \\
23 & 0.1 & 238 & 1.00 \\
24 & 0.5 & 49.5 & 1.00 \\
25 & 1 & 26.5 & 1.00 \\
26 & 10 & 4.1 & 1.00 \\
27 & 50 & 1.5 & 1.00 \\
28 & 100 & 1.07 & 1.00 \\
29 & 500 & 0.55 & 1.00 \\
30 & 1000 & 0.46 & 1.00 \\
31 & 5000 & 0.39 & 1.00 \\
32 & 10000 & 0.41 & 1.00 \\
33 & 50000 & 0.49 & 1.00 \\
34 & 100000 & 0.48 & 1.00 \\
35 & 200000 & 0.4 & 1.00 \\
36 & 300000 & 0.18 & 1.00 \\
37 & 500000 & 0.09 & 1.00 \\
38 & 600000 & 0.1 & 1.00 \\
39 & 700000 & 0.15 & 1.00 \\
40 & 1000000 & 0.2 & 1.00
\end{tabular}

Source: (Cheng, 2009)

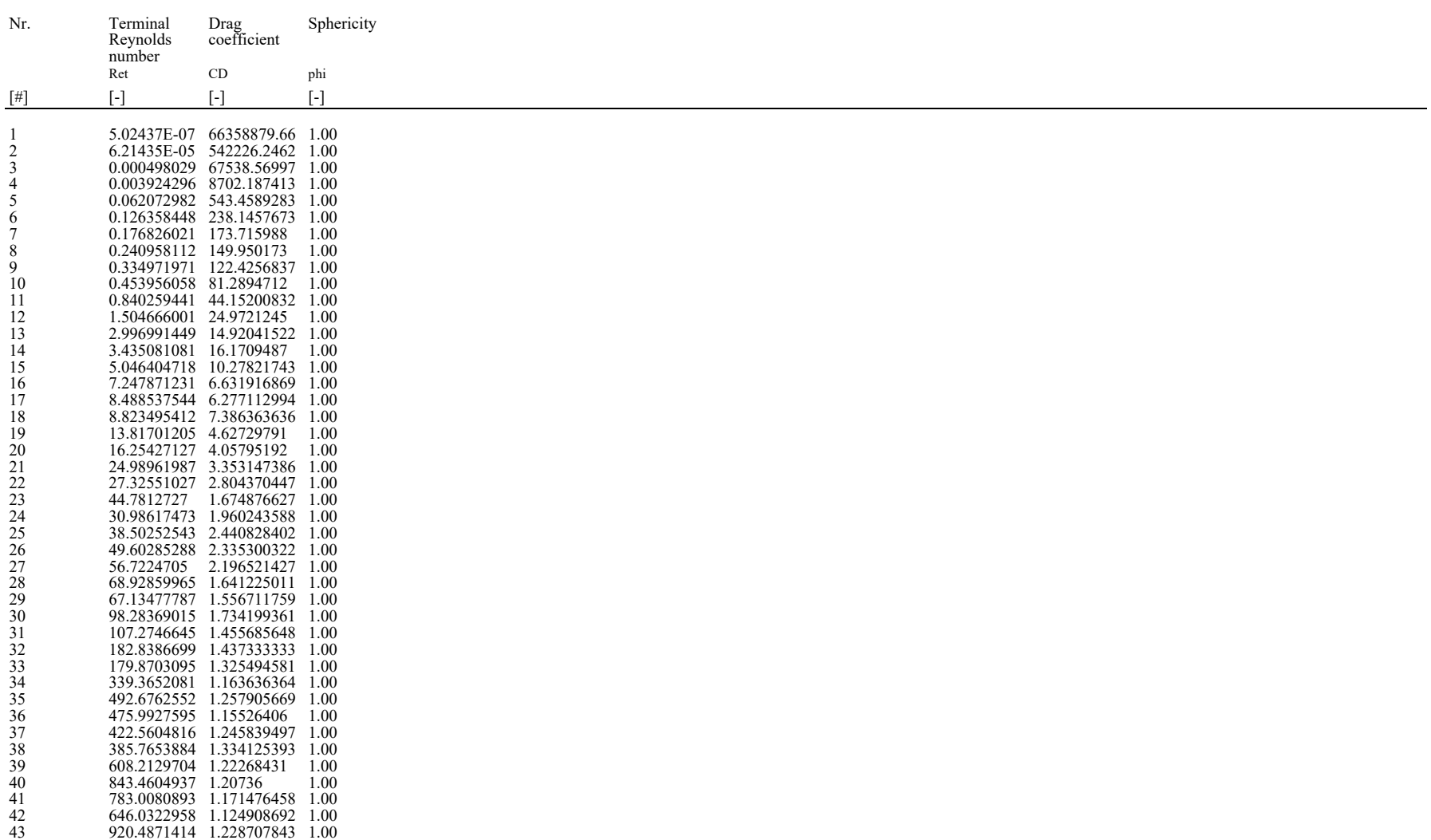

\section{Source: (Wu et al., 2006)}

\begin{tabular}{llll} 
Nr. & $\begin{array}{l}\text { Terminal } \\
\text { Reynolds } \\
\text { number } \\
\text { Ret }\end{array}$ & $\begin{array}{l}\text { Drag } \\
\text { coefficient }\end{array}$ & Sphericity \\
{$[\#]$} & {$[-]$} & CD & phi \\
{$[-]$} & {$[-]$} \\
\hline 1 & 1700 & 2.482819 & 0.63 \\
2 & 1430 & 1.489691 & 0.80
\end{tabular}




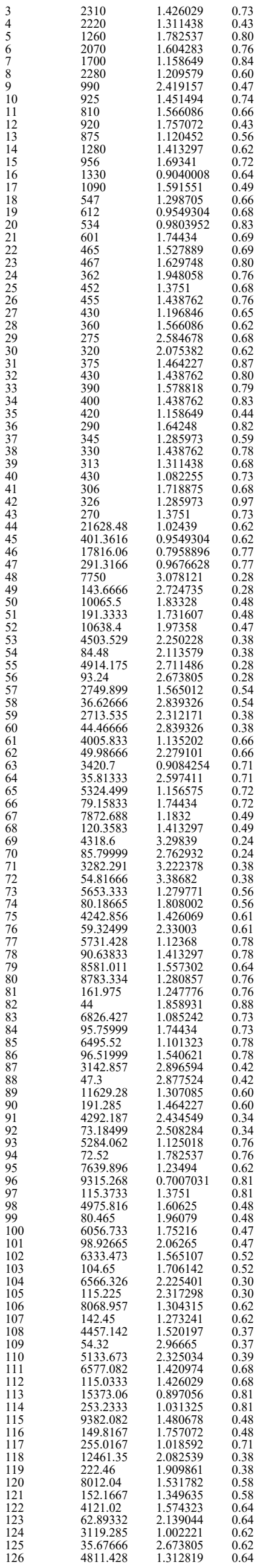




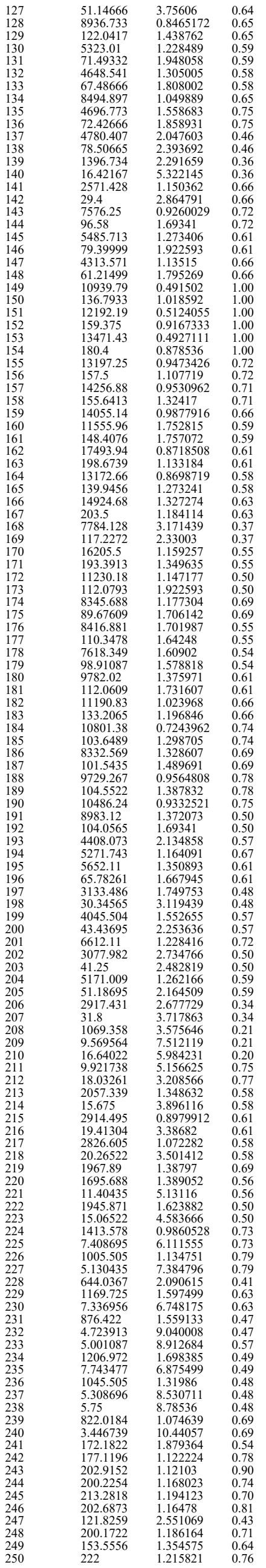




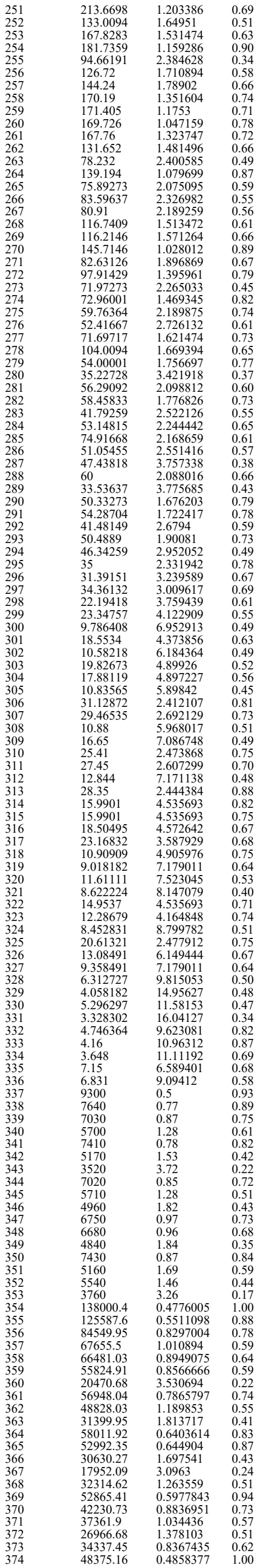




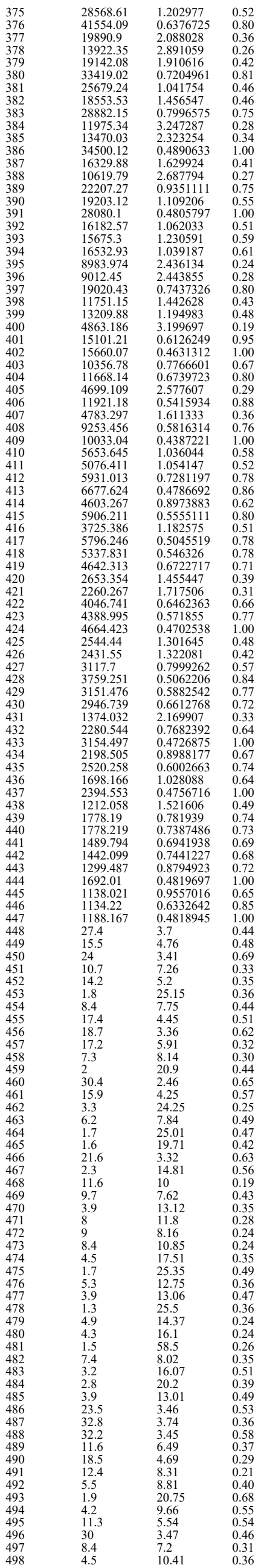




\begin{tabular}{|c|c|c|c|}
\hline 499 & 1.5 & 20.71 & 0.56 \\
\hline 500 & 3.6 & 13 & 0.27 \\
\hline 501 & 1.4 & 25.1 & 0.31 \\
\hline 502 & 7.7 & 6.5 & 0.61 \\
\hline 503 & 8.2 & 7.6 & 0.46 \\
\hline 504 & 8.6 & 7.25 & 0.42 \\
\hline 505 & 21.8 & 4.25 & 0.58 \\
\hline 506 & 27.2 & 2.96 & 0.61 \\
\hline 507 & 28.9 & 3.24 & 0.59 \\
\hline 508 & 39.2 & 2.28 & 0.76 \\
\hline 509 & 8.6 & 6.1 & 0.45 \\
\hline 510 & 20.5 & 4.14 & 0.52 \\
\hline 511 & 17.1 & 4.39 & 0.36 \\
\hline 512 & 24.8 & 3.72 & 0.58 \\
\hline 513 & 9.1 & 6.52 & 0.51 \\
\hline 514 & 38.5 & 2.32 & 0.63 \\
\hline 515 & 31.8 & 2.99 & 0.65 \\
\hline 516 & 31.9 & 2.99 & 0.52 \\
\hline 517 & 17.1 & 4.16 & 0.51 \\
\hline 518 & 19.1 & 3.9 & 0.50 \\
\hline 519 & 13.5 & 4.56 & 0.50 \\
\hline 520 & 15.6 & 6.46 & 0.29 \\
\hline 521 & 11.6 & 7.02 & 0.33 \\
\hline 522 & 4.5 & 8.85 & 0.62 \\
\hline 523 & 7 & 7.78 & 0.42 \\
\hline 524 & 6.7 & 8 & 0.38 \\
\hline 525 & 37.6 & 2.72 & 0.55 \\
\hline 526 & 28.8 & 2.89 & 0.55 \\
\hline 527 & 36.6 & 3.47 & 0.39 \\
\hline 528 & 17 & 3.34 & 0.88 \\
\hline 529 & 11 & 6.8 & 0.28 \\
\hline 530 & 7.6 & $\begin{array}{l}0.0 \\
7.6\end{array}$ & $\begin{array}{l}0.47 \\
0.20\end{array}$ \\
\hline 531 & 21.8 & 4.91 & 0.32 \\
\hline 532 & 13.5 & 4.8 & 0.50 \\
\hline 533 & 4.2 & 12.31 & 0.55 \\
\hline 534 & 21.5 & 3.68 & 0.59 \\
\hline 535 & 6.2 & 8.33 & 0.65 \\
\hline 536 & 11.7 & 5.65 & 0.51 \\
\hline 537 & 4.1 & 14.35 & 0.51 \\
\hline 538 & 5.3 & 8.95 & 0.62 \\
\hline 539 & 10.4 & 5.75 & 0.58 \\
\hline 540 & 5.3 & 9.77 & 0.58 \\
\hline 541 & 43.8 & 2.87 & 0.50 \\
\hline 542 & 41.8 & 2.55 & 0.64 \\
\hline 543 & 18.1 & 5.44 & 0.69 \\
\hline 544 & 27 & 3.66 & 0.45 \\
\hline 545 & 3.9 & 11.68 & 0.53 \\
\hline 546 & 2.4 & 13.45 & 0.57 \\
\hline 547 & 1.8 & 25.7 & 0.40 \\
\hline 548 & 1.8 & 25.6 & 0.40 \\
\hline 549 & 2.4 & 19.29 & 0.37 \\
\hline 550 & 3.8 & 12.37 & 0.42 \\
\hline 551 & 1.8 & 28.39 & 0.32 \\
\hline 552 & 2.6 & 13.35 & 0.85 \\
\hline 553 & 4 & 16.62 & 0.35 \\
\hline 554 & 0.8 & 37.19 & 0.58 \\
\hline 555 & 22.6 & 3.45 & 0.62 \\
\hline 556 & 18.6 & 4.2 & 0.52 \\
\hline 557 & 15.9 & 4.88 & 0.48 \\
\hline 558 & 24.6 & 3.78 & 0.49 \\
\hline 559 & 21.6 & 4.13 & 0.56 \\
\hline 560 & 30.4 & 2.69 & 0.60 \\
\hline 561 & 19.8 & 4.37 & 0.40 \\
\hline 562 & 24.7 & 3.69 & 0.53 \\
\hline 563 & 10.9 & 5.98 & 0.47 \\
\hline 564 & 10.5 & 5.56 & 0.61 \\
\hline 565 & 10.4 & 6.89 & 0.47 \\
\hline 566 & 5 & 10.07 & 0.58 \\
\hline 567 & 4.4 & 15.35 & 0.33 \\
\hline 568 & 3.5 & 10.9 & 0.52 \\
\hline 569 & 4.8 & 9 & 0.80 \\
\hline 570 & 3.6 & 15.55 & 0.24 \\
\hline 571 & 2.2 & 16.77 & 0.45 \\
\hline
\end{tabular}

Source: (Dioguardi et al., 2018)

$\begin{array}{llll}0.032076364 & 1860.372569 & 0.58\end{array}$ $\begin{array}{lll}0.048192011 & 1467.40357 & 0.5 \\ 0.076095136 & 618.7473442 & 0.69\end{array}$ $\begin{array}{llll}0.142769061 & 286.8478685 & 0.75\end{array}$ $\begin{array}{llll}0.137125397 & 301.3033679 & 0.67\end{array}$ $\begin{array}{llll}0.161338523 & 280.3993143 & 0.64\end{array}$ $\begin{array}{llll}0.206780302 & 244.462989 & 0.65\end{array}$ $\begin{array}{lll}0.201949638 & 209.1531874 & 0.54\end{array}$ $\begin{array}{llll}0.209362575 & 240.8064663 & 0.66\end{array}$ $\begin{array}{llll}0.248184032 & 158.1944031 & 0.60 \\ 0.218300627 & 181.8579297 & 0.61\end{array}$ $\begin{array}{llll}0.377094218 & 133.381916 & 0.71\end{array}$ $0.547637189 \quad 95.34828835 \quad 0.76$ $\begin{array}{lllll}0.674406484 & 62.65470259 & 0.85\end{array}$ $\begin{array}{llll}0.347950744 & 118.6276563 & 0.57\end{array}$ $\begin{array}{llll}0.612150494 & 90.11383489 & 0.71\end{array}$ $\begin{array}{llll}0.802209723 & 59.42103546 & 0.83\end{array}$ $\begin{array}{llll}0.771808955 & 67.67445937 & 0.72\end{array}$ $\begin{array}{llll}1.131706077 & 52.57866321 & 0.85\end{array}$ $\begin{array}{llll}0.566782588 & 76.85856294 & 0.60 \\ 0.745707769 & 55.18965316 & 0.67\end{array}$ $\begin{array}{llll}0.74570769 & 55.18965316 & 0.67 \\ 1.348184921 & 37.825806 & 0.93\end{array}$ $\begin{array}{llll}0.581725095 & 90.62815846 & 0.49\end{array}$ $\begin{array}{llll}0.58725095 & 90.62815846 & 0.49 \\ 1.277208947 & 49.93482608 & 0.75\end{array}$ $\begin{array}{lllll}0.666933462 & 52.06184147 & 0.64\end{array}$ $\begin{array}{llll}0.600917611 & 115.0541753 & 0.44\end{array}$ $\begin{array}{llll}0.992571085 & 54.28447021 & 0.67\end{array}$ $\begin{array}{llll}1.380108657 & 39.63274182 & 0.8\end{array}$ $\begin{array}{llll}0.993136536 & 61.86665431 & 0.56\end{array}$ $\begin{array}{llll}0.786311038 & 55.5231404 & 0.64\end{array}$ $\begin{array}{llll}1.421567227 & 41.85678858 & 0.72 \\ 1.027925166 & 66.65136977 & 0.57\end{array}$ $\begin{array}{llll}1.027925166 & 66.65136977 & 0.57\end{array}$ $\begin{array}{llll}0.754238805 & 64.6532319 & 0.43\end{array}$ $\begin{array}{llll}057325609 & 37.57426252 & 0.66\end{array}$ $\begin{array}{llll}1.005140243 & 53.41980972 & 0.54\end{array}$ $\begin{array}{llll}1.058259266 & 42.04969592 & 0.60\end{array}$ $\begin{array}{llll}1.665886499 & 37.23255335 & 0.65\end{array}$ $\begin{array}{lll}1.97404242 & 27.34220547 & 0.67\end{array}$ $\begin{array}{lll}0.88306405 & 102.1248142 & 0.36\end{array}$ $\begin{array}{lll}1.7974689 & 31.5963232 & 0.64\end{array}$ $\begin{array}{llll}1.063942151 & 34.37217736 & 0.51 \\ 1.900012127 & 25.50669919 & 0.67\end{array}$ $\begin{array}{lll}1.900012127 & 25.50669919 & 0.67 \\ .497209605 & 26.50192529 & 0.70\end{array}$ $\begin{array}{lll}2.497209605 & 26.50192529 & 0.70 \\ 1.316595384 & 37.66859784 & 0.52\end{array}$ 


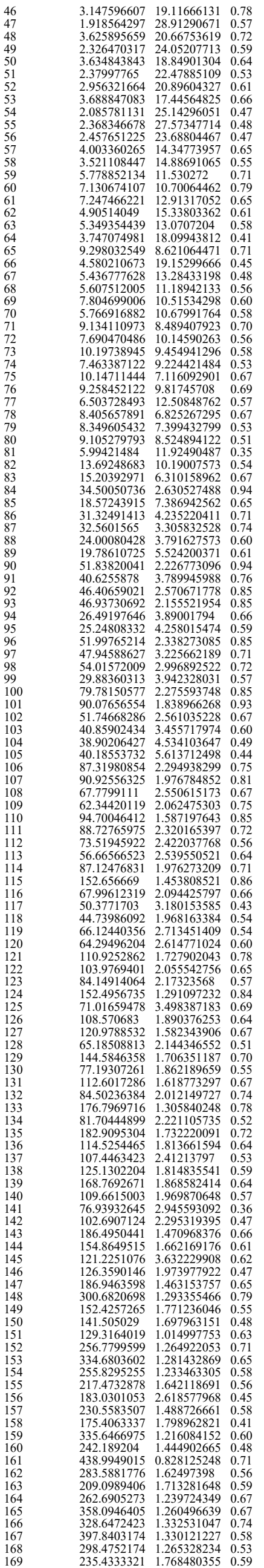




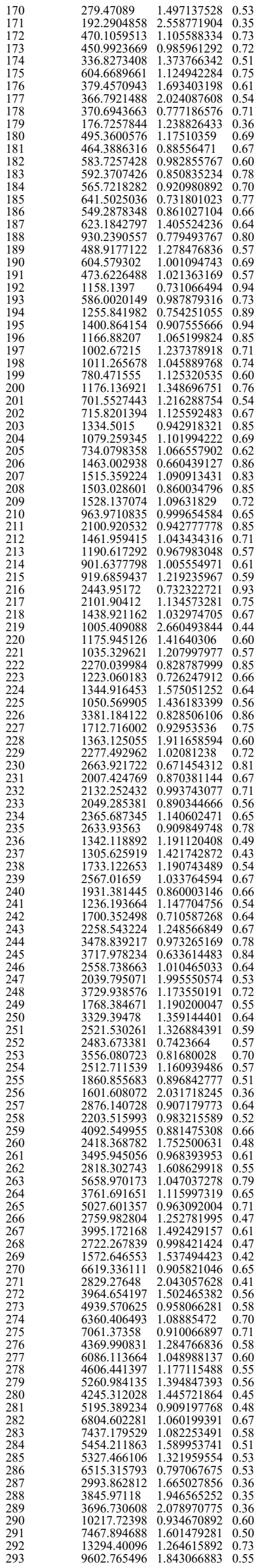




$\begin{array}{llll}294 & 13756.18392 & 1.487925923 & 0.75 \\ 295 & 11913.25285 & 1.463552095 & 0.64 \\ 296 & 20606.1022 & 2.09507434 & 0.55 \\ 297 & 10483.01456 & 1.566321536 & 0.37 \\ 298 & 14242.83193 & 1.772478256 & 0.44 \\ 299 & 17185.62015 & 1.655987669 & 0.48 \\ 300 & 21391.59039 & 1.724811783 & 0.46 \\ 301 & 19146.78111 & 1.575007493 & 0.38 \\ 302 & 17857.80707 & 1.62054788 & 0.35 \\ 303 & 20704.96363 & 1.194655778 & 0.33\end{array}$

Source: (Breakey et al., 2018)

$\begin{array}{lll}2.097303544 & 13.92404322 & 1.00 \\ 1.156013908 & 27.14865788 & 0.90\end{array}$ $\begin{array}{lll}1.156013908 & 27.14865788 & 0.90 \\ 0.886331418 & 29.95849533 & 1.00\end{array}$ $\begin{array}{lll}0.886331418 & 29.95849533 & 1.00 \\ 1.316579366 & 21.01233001 & 0.92\end{array}$ $\begin{array}{llll}1.04352583 & 26.05222855 & 0.95\end{array}$ $\begin{array}{llll}0.863198876 & 33.46942907 & 0.78\end{array}$ $\begin{array}{llll}0.86319876 & 33.46942907 & 0.98 \\ 1.703538147 & 16.17068677 & 0.87\end{array}$ $\begin{array}{llll}0.854493637 & 32.73739373 & 1.00\end{array}$ $\begin{array}{llll}0.634834252 & 43.27017058 & 1.00\end{array}$ $\begin{array}{llll}0.547401406 & 51.85646815 & 0.85\end{array}$ $\begin{array}{llll}2.215022715 & 14.97425544 & 0.89\end{array}$ $\begin{array}{lll}1.627316473 & 20.09807323 & 0.83\end{array}$ $\begin{array}{llll}0.787322907 & 42.83869179 & 0.74\end{array}$ $\begin{array}{llll}1.381100694 & 20.72269714 & 0.79\end{array}$ $\begin{array}{llll}1.223821628 & 25.18911004 & 0.85\end{array}$ $\begin{array}{llll}0.630746406 & 42.08666625 & 0.86 \\ 0.595170079 & 56.23391778 & 0.82 \\ 0.73053526 & 38.28979126 & 0.95\end{array}$ $\begin{array}{llll}0.730453526 & 38.28979126 & 0.95\end{array}$ $\begin{array}{llll}0.470309622 & 54.09771434 & 0.95\end{array}$ $\begin{array}{llll}0.581194437 & 52.80097495 & 0.75\end{array}$ $\begin{array}{lll}0.083537611 & 339.27868 & 0.58\end{array}$ $\begin{array}{llll}0.081066598 & 345.1757562 & 0.80\end{array}$ $\begin{array}{llll}0.654286038 & 41.26548312 & 0.89\end{array}$ $\begin{array}{llll}0.106338487 & 264.5487431 & 0.74\end{array}$ $\begin{array}{llll}0.366675057 & 67.69810132 & 1.00\end{array}$ $\begin{array}{llll}0.391090837 & 66.18310329 & 1.00 \\ 0.439525677 & 59.88607556 & 0.95\end{array}$ $\begin{array}{llll}0.4588997 & 55.886075963 & 0.95\end{array}$ $\begin{array}{llll}0.092795112 & 289.7788349 & 0.97\end{array}$ $\begin{array}{llll}1.0848208 & 26.74252532 & 0.91\end{array}$ $\begin{array}{llll}0.704806251 & 40.01406894 & 0.87\end{array}$ $\begin{array}{llll}0.615245112 & 46.48144647 & 0.85\end{array}$ $\begin{array}{llll}0.430210184 & 63.65665982 & 1.00\end{array}$ $\begin{array}{llll}0.645900529 & 46.18408823 & 0.88\end{array}$ $\begin{array}{llll}0.417413032 & 65.82952334 & 0.93\end{array}$ $\begin{array}{llll}0.407272878 & 60.34453129 & 1.00\end{array}$ $\begin{array}{llll}0.452389519 & 59.64613115 & 0.90\end{array}$ $\begin{array}{llll}0.456978276 & 60.15154802 & 0.89\end{array}$ $\begin{array}{llll}0.307958748 & 85.06117387 & 0.90\end{array}$ $\begin{array}{llll}0.206811564 & 118.6690833 & 0.90\end{array}$ $\begin{array}{llll}0.262135076 & 94.49730025 & 0.8\end{array}$ $\begin{array}{llll}0.72159074 & 37.13955231 & 0.95\end{array}$ $\begin{array}{llll}0.595367516 & 41.03753705 & 0.80\end{array}$ $\begin{array}{llll}0.320110837 & 77.75704716 & 0.95\end{array}$ $\begin{array}{llll}0.242052412 & 99.45513037 & 0.85\end{array}$ $\begin{array}{llll}0.486896396 & 57.591364 & 1.00\end{array}$ $\begin{array}{llll}0.148869529 & 173.3651743 & 0.83 \\ 0.406866406 & 66.88851676 & 0.85\end{array}$ $\begin{array}{llll}0.406866406 & 66.88851676 & 0.85 \\ 0.321321709 & 78.68259661 & 0.98\end{array}$ $\begin{array}{llll}0.321321709 & 78.68259661 & 0.98\end{array}$ $0.098103511 \quad 3373421257 \quad 0.76$ $\begin{array}{llll}0.156437234 & 163.9496063 & 0.78\end{array}$ $\begin{array}{llll}0.073743047 & 419.7473094 & 0.87\end{array}$ $\begin{array}{llll}0.074747601 & 367.9405763 & 0.90\end{array}$ $\begin{array}{llll}0.164946364 & 176.6519369 & 0.92\end{array}$ $\begin{array}{llll}0.122479897 & 225.8772415 & 1.00\end{array}$ $\begin{array}{lll}0.115557859 & 214.4650829 & 0.96\end{array}$ $\begin{array}{llll}0.1362276 & 191.7554459 & 0.94\end{array}$ $\begin{array}{llll}0.106039871 & 249.6496745 & 0.96\end{array}$ $\begin{array}{llll}0.141352596 & 284.5678382 & 0.74\end{array}$ $\begin{array}{llll}0.057211042 & 524.118945 & 1.00\end{array}$ $\begin{array}{llll}0.119873439 & 245.673104 & 0.77\end{array}$ $\begin{array}{llll}0.110213552 & 273.1178318 & 0.83\end{array}$ $\begin{array}{llll}0.101297445 & 268.0457631 & 0.89\end{array}$ $\begin{array}{lll}0.132652966 & 178.8641246 & 0.99\end{array}$ $\begin{array}{llll}0.065299679 & 415.6158689 & 0.84\end{array}$ $\begin{array}{llll}0.059205269 & 444.9140893 & 0.9\end{array}$ $\begin{array}{llll}0.038662824 & 749.2800107 & 0.61\end{array}$ $\begin{array}{llll}0.066457504 & 381.9997785 & 0.84\end{array}$ $\begin{array}{llll}2221.340379 & 1.281584386 & 0.77 \\ 1701.10559 & 2.442737869 & 0.86\end{array}$ $\begin{array}{llll}2311.557919 & 1.39706265 & 0.96\end{array}$ $\begin{array}{llll}2071.342152 & 1.640150944 & 0.99\end{array}$ $\begin{array}{llll}1561.084632 & 3.206258668 & 0.85\end{array}$ $\begin{array}{llll}2281.351173 & 1.181549648 & 1.00\end{array}$ $\begin{array}{llll}990.3071117 & 2.375647899 & 0.80\end{array}$ $\begin{array}{lll}1700.666848 & 1.145281719 & 0.93\end{array}$ $\begin{array}{lll}1760.439063 & 1.74581763 & 1.00\end{array}$ $\begin{array}{llll}1430.526392 & 1.471520734 & 0.95\end{array}$ $\begin{array}{llll}920.2091031 & 1.7256885 & 0.80\end{array}$ $\begin{array}{llll}1330.2920463 & 1.573261285 & 0.80\end{array}$ $\begin{array}{llll}1280.389836 & 1.382471338 & 0.95\end{array}$ $\begin{array}{llll}810.1444744 & 1.547881164 & 0.79\end{array}$ $\begin{array}{llll}925.1874098 & 1.436834764 & 0.81\end{array}$ $\begin{array}{llll}465.0389101 & 1.509708744 & 0.56\end{array}$ $\begin{array}{llll}452.0340546 & 1.365132775 & 0.73\end{array}$ $\begin{array}{lll}547.0509604 & 1.281634916 & 0.65\end{array}$ $\begin{array}{llll}534.0399272 & 0.96695514 & 0.67\end{array}$ $\begin{array}{llll}375.0229675 & 1.453712459 & 0.58\end{array}$ $\begin{array}{llll}612.0541523 & 0.947704309 & 0.70\end{array}$ $\begin{array}{llll}390.0260596 & 1.531562126 & 0.68\end{array}$ $\begin{array}{lllll}4 & 0.04523 & 1.605783095 & 0.67\end{array}$ $\begin{array}{llll}320.0200021 & 2.058268027 & 0.54\end{array}$ $\begin{array}{llll}601.0773603 & 1.723996357 & 0.83\end{array}$ $\begin{array}{llll}275.0161391 & 2.535557963 & 0.67\end{array}$ $\begin{array}{llll}430.0276802 & 1.19131473 & 0.91\end{array}$ $\begin{array}{lll}400.0259955 & 1.396520186 & 0.70\end{array}$ $\begin{array}{llll}455.0355672 & 1.423727227 & 0.66\end{array}$ $\begin{array}{llll}345.0173521 & 1.278185515 & 0.75\end{array}$ $\begin{array}{llll}326.0152371 & 1.282410865 & 0.74\end{array}$ $\begin{array}{llll}3 & 326.0158152 & 1.692453676 & 0.97\end{array}$ $\begin{array}{lll}430.0257888 & 1.071335621 & 1.00 \\ 360.0218203 & 1.552925453 & 0.69\end{array}$ 
$\begin{array}{llll}430.0311579 & 1.422698178 & 0.83\end{array}$ $\begin{array}{lll}290.013345 & 1.58287952 & 0.76\end{array}$ $\begin{array}{lll}0.050728174 & 1.526 .253434 & 0.32\end{array}$ $\begin{array}{llll}0.063602786 & 652.8310609 & 0.32\end{array}$ $\begin{array}{llll}0.074716767 & 473.0607852 & 0.32\end{array}$ $\begin{array}{llll}0.063050915 & 704.8870055 & 0.44\end{array}$ $\begin{array}{llll}0.073209117 & 522.8437878 & 0.44\end{array}$ $\begin{array}{llll}0.091423824 & 335.2613793 & 0.44\end{array}$ $\begin{array}{llll}0.037042298 & 1277.908392 & 0.29 \\ 0.045882767 & 832.9067095 & 0.29\end{array}$ $\begin{array}{llll}0.045882767 & 832.9067095 & 0.29\end{array}$ 0.048405173748 .95518660 .33 $\begin{array}{llll}0.037278837 & 1264.810504 & 0.36\end{array}$ $\begin{array}{llll}0.047459108 & 780.3887948 & 0.36\end{array}$ $\begin{array}{llll}0.052585863 & 635.6413663 & 0.36\end{array}$ $\begin{array}{llll}0.07877835 & 525.254103 & 0.50\end{array}$ $\begin{array}{lll}0.095213142 & 359.574944 & 0.50\end{array}$ $\begin{array}{llll}0.111512108 & 262.1435399 & 0.50\end{array}$ $\begin{array}{llll}0.05212982 & 813.3968675 & 0.46\end{array}$ $\begin{array}{llll}0.060603218 & 601.8435054 & 0.46\end{array}$ $\begin{array}{llll}0.074050132 & 403.1097292 & 0.46\end{array}$ $\begin{array}{llll}0.068837856 & 448.7008435 & 0.55\end{array}$ $0.076545569 \quad 362.8870293 \quad 0.55$ $\begin{array}{llll}0.03510189 & 1088.719338 & 0.56\end{array}$ $\begin{array}{llll}0.037952521 & 931.3130279 & 0.56\end{array}$ $\begin{array}{llll}0.047845888 & 585.986627 & 0.56\end{array}$ $\begin{array}{llll}0.059840103 & 604.0109206 & 0.59\end{array}$ $\begin{array}{llll}0.065517703 & 503.862611 & 0.59\end{array}$ $\begin{array}{llll}0.079576521 & 341.5541448 & 0.59\end{array}$ $\begin{array}{llll}0.03998791 & 1054.786834 & 0.43 \\ 0.052708141 & 607.1090637 & 0.43\end{array}$ $\begin{array}{llll}0.052708141 & 607.1090637 & 0.43 \\ 0.065586383 & 501.2664829 & 0.63\end{array}$ $\begin{array}{llll}0.06586383 & 501.2664829 & 0.63\end{array}$ $\begin{array}{llll}0.059455226 & 546.2242188 & 0.65\end{array}$ $\begin{array}{llll}0.060822938 & 521.9347817 & 0.65\end{array}$ $\begin{array}{llll}0.074741413 & 345.6435159 & 0.65\end{array}$ $\begin{array}{lll}0.069686801 & 499.1204392 & 0.61\end{array}$ $\begin{array}{llll}0.071706708 & 471.3970286 & 0.6\end{array}$ $\begin{array}{llll}0.09079483 & 294.0249415 & 0.6\end{array}$ $\begin{array}{llll}0.070960399 & 471.7042747 & 0.64\end{array}$ $\begin{array}{llll}0.091248935 & 285.2635613 & 0.64\end{array}$ $\begin{array}{llll}0.09177738 & 335.2543297 & 0.69\end{array}$ $\begin{array}{llll}0.095189616 & 311.6495907 & 0.69\end{array}$ $\begin{array}{llll}0.100379318 & 258.4751891 & 0.64\end{array}$ $\begin{array}{llll}0.079977902 & 407.1620875 & 0.64\end{array}$ $\begin{array}{llll}0.082011891 & 303.8800652 & 0.68\end{array}$ $\begin{array}{llll}0.066852892 & 457.3150125 & 0.68\end{array}$ $\begin{array}{lll}0.098955219 & 253.4278248 & 0.72\end{array}$ $\begin{array}{llll}0.084272209 & 349.4322145 & 0.72\end{array}$ $\begin{array}{llll}0.084005436 & 368.1592525 & 0.72\end{array}$ $\begin{array}{llll}0.092557535 & 303.2682468 & 0.72\end{array}$ $\begin{array}{llll}0.10273345 & 246.1652552 & 0.72\end{array}$ $\begin{array}{llll}0.063463464 & 450.196888 & 0.74\end{array}$ $\begin{array}{llll}0.06633443 & 412.0709807 & 0.74\end{array}$ $\begin{array}{llll}0.070864617 & 403.6583391 & 0.76\end{array}$ $\begin{array}{llll}0.078550767 & 328.527675 & 0.76\end{array}$ $\begin{array}{llll}0.095699613 & 257.7401138 & 0.77\end{array}$ $\begin{array}{llll}0.086257515 & 317.2550188 & 0.77\end{array}$ $\begin{array}{llll}0.092523287 & 288.8534455 & 0.80\end{array}$ $\begin{array}{llll}0.096335493 & 266.4446518 & 0.80\end{array}$ $\begin{array}{llll}0.06638105 & 387.5073107 & 0.81\end{array}$ $\begin{array}{llll}0.289836828 & 99.01171342 & 0.83\end{array}$ $\begin{array}{llll}0.033885861 & 28.72968053 & 0.83 \\ 0.312770288 & 92.89832211 & 0.82\end{array}$ $\begin{array}{llll}1.03 & 0.146117621 & 200.1469513 & 0.82\end{array}$ $\begin{array}{llll}0.600299767 & 51.158517 & 0.78\end{array}$ $\begin{array}{llll}0.604103922 & 51.26294995 & 0.78\end{array}$ $\begin{array}{llll}0.407801864 & 75.55968208 & 0.77\end{array}$ $\begin{array}{lll}0.392531298 & 24.17138353 & 0.77\end{array}$ $\begin{array}{llll}0.426138364 & 73.43879755 & 0.76\end{array}$ $\begin{array}{lll}0.678742848 & 48.6453019 & 0.75\end{array}$ $\begin{array}{llll}0.456111655 & 71.80834157 & 0.75\end{array}$ $\begin{array}{llll}0.710160713 & 46.45523265 & 0.74\end{array}$ $\begin{array}{llll}0.791990174 & 168.1043597 & 0.74 \\ 1.570082477 & 22.95782654 & 0.74\end{array}$ $\begin{array}{lllll}1.570082477 & 22.95782654 & 0.74 \\ 0.091732108 & 349.4543336 & 0.74\end{array}$ $\begin{array}{llll}0.480639462 & 70.39202266 & 0.73\end{array}$ $\begin{array}{llll}0.754479125 & 44.73530363 & 0.73\end{array}$ $\begin{array}{lllll}0.505488172 & 66.59517954 & 0.73\end{array}$ $\begin{array}{llll}0.207732805 & 159.458341 & 0.72\end{array}$ $\begin{array}{llll}0.51169768 & 67.54427546 & 0.72\end{array}$ $\begin{array}{llll}0.235017277 & 143.3846972 & 0.71\end{array}$ $\begin{array}{llll}0.802681975 & 44.53601845 & 0.7\end{array}$ $\begin{array}{llll}0.110675682 & 313.9628455 & 0.69\end{array}$ $\begin{array}{llll}0.239054139 & 149.5517938 & 0.69\end{array}$ $\begin{array}{llll}0.114093142 & 305.3422623 & 0.69\end{array}$ $\begin{array}{llll}0.26426388 & 143.2602329 & 0.66 \\ 0.138963406 & 271.0334562 & 0.64\end{array}$ $\begin{array}{llll}0.160097489 & 265.3607587 & 0.60\end{array}$ $\begin{array}{llll}0.184897589 & 251.4605599 & 0.56\end{array}$ $\begin{array}{llll}7.083483494 & 5.346309561 & 0.59\end{array}$ $\begin{array}{llll}2.649816928 & 6.777654832 & 0.55\end{array}$ $\begin{array}{llll}9.822597838 & 4.337302832 & 0.52\end{array}$ $\begin{array}{llll}3.965126845 & 6.21119694 & 0.44\end{array}$ $\begin{array}{llll}0.333359505 & 109.3362821 & 0.43\end{array}$ $\begin{array}{llll}0.382684989 & 88.15279191 & 0.42\end{array}$ $\begin{array}{llll}0.193763479 & 163.9240198 & 0.40\end{array}$ $\begin{array}{llll}0.241690143 & 138.4586384 & 0.39\end{array}$ $\begin{array}{llll}0.217273725 & 147.7507466 & 0.38\end{array}$ $\begin{array}{llll}0.937124323 & 47.48600588 & 0.65\end{array}$ $\begin{array}{llll}1.537326067 & 35.29052143 & 0.69\end{array}$ $\begin{array}{llll}1.807762843 & 31.90194482 & 0.68\end{array}$ $\begin{array}{llll}2.168030094 & 26.61648518 & 0.68\end{array}$ $\begin{array}{llll}2.556806665 & 22.32710048 & 0.68\end{array}$ $\begin{array}{lll}2.782682648 & 21.54233095 & 0.65\end{array}$ $\begin{array}{llll}0.000120472 & 267491.176 & 0.69\end{array}$ $\begin{array}{llll}0.002784372 & 11210.9961 & 0.69\end{array}$ $\begin{array}{llll}0.171713069 & 185.0145403 & 0.69\end{array}$

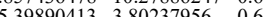
$\begin{array}{llll}189.883489 & 2.8237956 & 0.69\end{array}$ $\begin{array}{llll}0.272876866 & 106.7370653 & 0.69\end{array}$ $\begin{array}{llll}0.000193768 & 170038.3827 & 0.69\end{array}$ $\begin{array}{llll}0.004509785 & 7027.551149 & 0.69\end{array}$ $\begin{array}{llll}0.839363673 & 8.525683664 & 0.69\end{array}$ $\begin{array}{llll}35.10609393 & 3.272942176 & 0.69\end{array}$ $\begin{array}{llll}236.1845746 & 2.499846719 & 0.69\end{array}$ $\begin{array}{llll}0.000448152 & 75064.28336 & 0.69\end{array}$ 


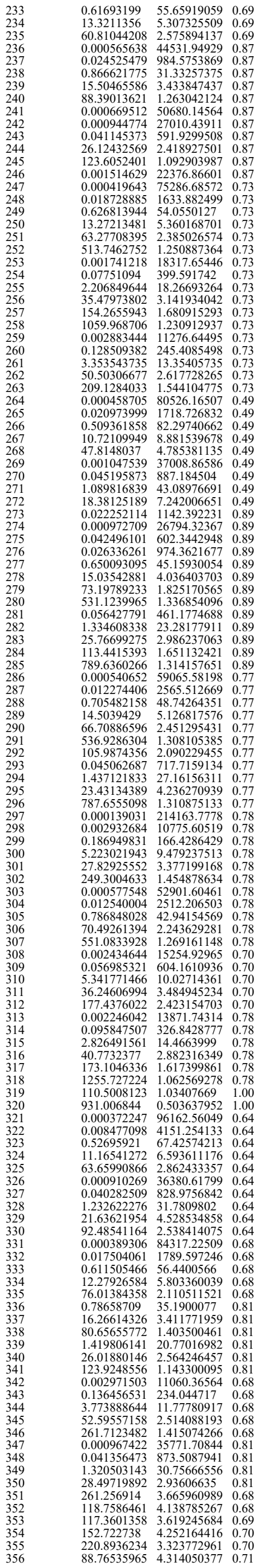




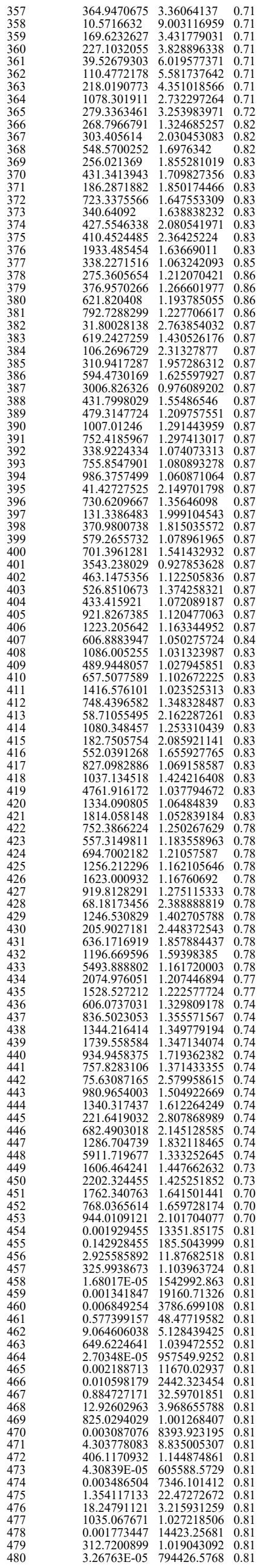




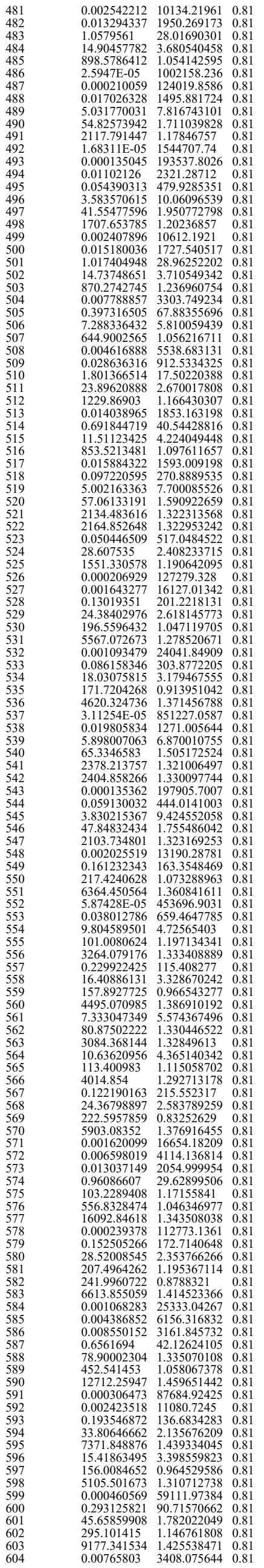




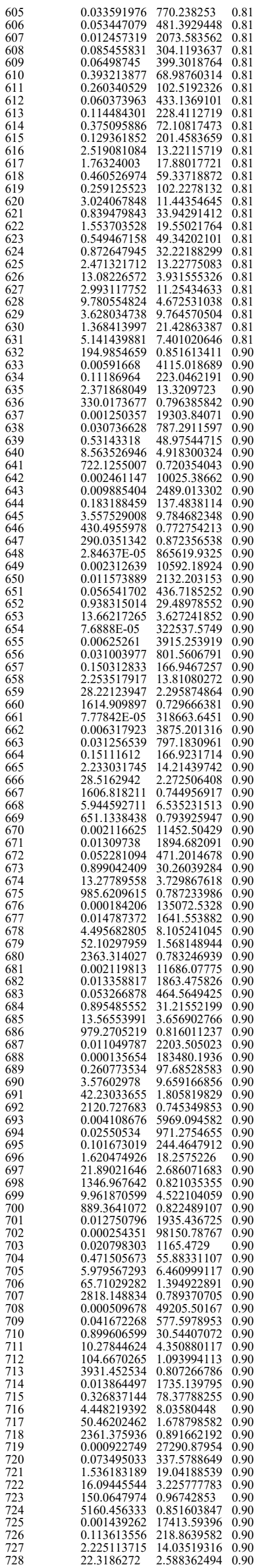




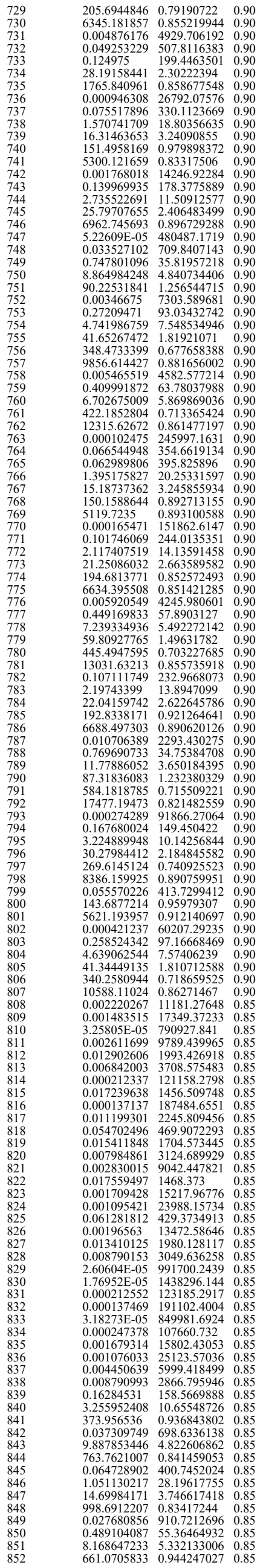




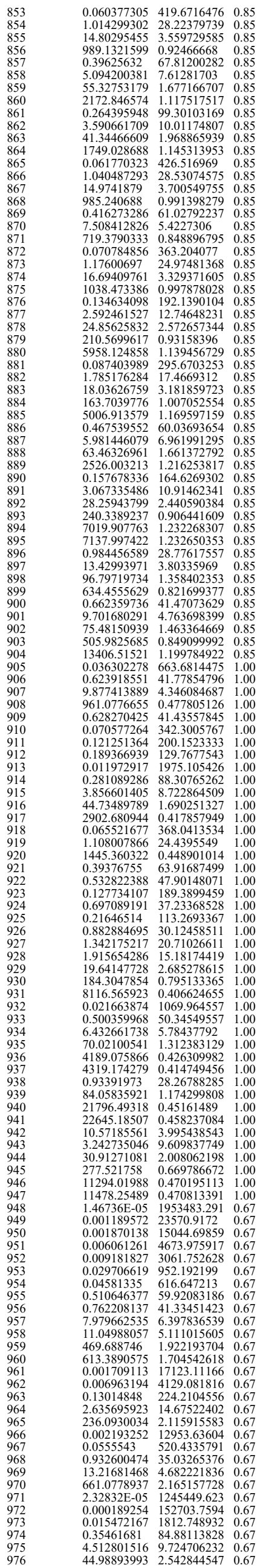




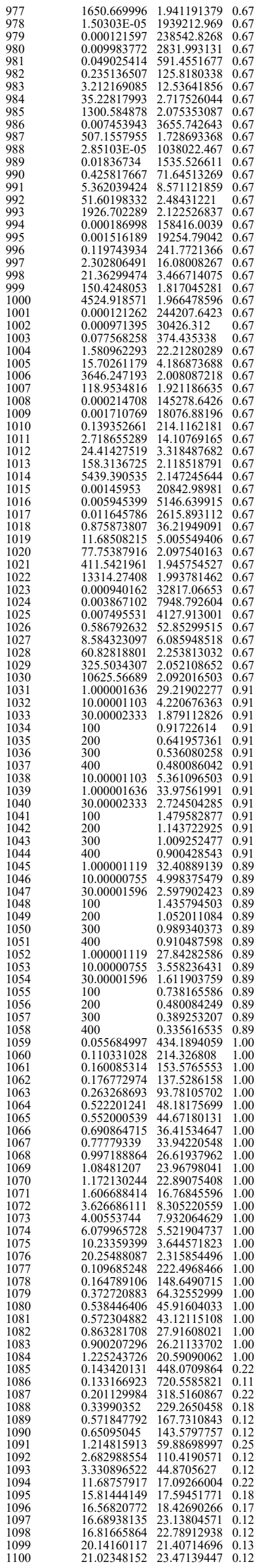


$\begin{array}{lll}21.40347591 & 25.35406942 & 0.11 \\ 23.00715728 & 27.58168759 & 0.12\end{array}$ $\begin{array}{llll}24.8075728 & 27.58168759 & 0.12\end{array}$ $\begin{array}{llll}41.86601113 & 14.10711086 & 0.22\end{array}$ $\begin{array}{lll}42.6075222 & 13.88430039 & 0.22\end{array}$ $\begin{array}{llll}54.52611249 & 20.64359248 & 0.16\end{array}$ $\begin{array}{llll}63.63120092 & 24.60945299 & 0.14\end{array}$ $\begin{array}{llll}69.49967678 & 28.4745755 & 0.13\end{array}$ $\begin{array}{llll}72.94921068 & 31.39255606 & 0.12\end{array}$ $\begin{array}{llll}26.25464532 & 24.80004509 & 0.13\end{array}$ $\begin{array}{llll}58.1886016 & 14.6732521 & 0.22 \\ 58.98960964 & 16.87059012 & 0.19\end{array}$ $\begin{array}{lllll}25.78731309 & 16.54222201 & 0.18\end{array}$ $\begin{array}{lll}25.78731309 & 16.54222201 & 0.18 \\ 30.28424599 & 20.97092676 & 0.15\end{array}$ $\begin{array}{llll}37.89171673 & 22.39696809 & 0.13\end{array}$ $\begin{array}{llll}146.3696514 & 1.033520215 & 0.89\end{array}$ $\begin{array}{llll}3.370920854 & 16.14188422 & 0.60\end{array}$ $\begin{array}{llll}4.024068768 & 14.72566607 & 0.80\end{array}$ $\begin{array}{llll}5.460648306 & 11.85105849 & 0.70\end{array}$ $\begin{array}{llll}6.4228202 & 9.909173043 & 0.78\end{array}$ $\begin{array}{llll}7.000481503 & 9.251878616 & 0.86 \\ 11.43754977 & 6.17752277 & 0.76\end{array}$ $\begin{array}{llll}11.43754977 & 6.177522277 & 0.76 \\ 7.399947706 & 6.774516018 & 0.85\end{array}$ $\begin{array}{llll}4.91056217 & 9.888793502 & 0.87\end{array}$ $\begin{array}{llll}11.48432773 & 6.250458939 & 0.80\end{array}$ $\begin{array}{lll}11.48432773 & 6.250458939 & 0.80 \\ 11.81917509 & 7.589138468 & 0.88\end{array}$ $\begin{array}{llll}13.07280063 & 7.235641568 & 0.74\end{array}$ $\begin{array}{llll}20.20613359 & 4.948275629 & 0.89\end{array}$ $\begin{array}{llll}13.31774517 & 6.204991476 & 0.87\end{array}$ $\begin{array}{llll}18.80368649 & 4.395341306 & 0.85\end{array}$ $\begin{array}{lllll}18.75463632 & 4.595079354 & 0.87\end{array}$ $\begin{array}{llll}18.75463632 & 4.595079354 & 0.87 \\ 15.21774801 & 2.502503771 & 0.83\end{array}$ $\begin{array}{llll}15.21774901 & 4.577896565 & 0.75\end{array}$ $\begin{array}{llll}15.27136632 & 4.578503262 & 0.79\end{array}$ $\begin{array}{llll}25.74482005 & 2.48755869 & 0.96\end{array}$ $\begin{array}{llll}23.70564592 & 4.148262975 & 0.79\end{array}$ $\begin{array}{llll}27.96592048 & 2.635146917 & 1.00\end{array}$ $\begin{array}{llll}34.12967038 & 3.801778936 & 0.84\end{array}$ $\begin{array}{llll}35.84841991 & 3.445966188 & 0.68\end{array}$ $\begin{array}{llll}23.60775023 & 3.625025945 & 0.90\end{array}$ $\begin{array}{llll}47.74460698 & 3.735932827 & 0.77\end{array}$ $\begin{array}{llll}28.88194966 & 2.470643347 & 0.91\end{array}$ $\begin{array}{llll}29.9099407 & 2.709997653 & 0.82\end{array}$ $\begin{array}{llll}42.52147686 & 2.540810217 & 0.84 \\ 31.84637935 & 3.255395185 & 0.85\end{array}$ $\begin{array}{llll}4.56185956 & 2.933981877 & 0.83\end{array}$ $\begin{array}{lllll}51.20113876 & 1.689241754 & 0.86\end{array}$ $\begin{array}{llll}31.59677399 & 2.428376835 & 0.81\end{array}$ $\begin{array}{llll}35.41975498 & 2.339269278 & 0.91\end{array}$ $\begin{array}{llll}41.91310263 & 2.679721602 & 0.83\end{array}$ $\begin{array}{llll}34.83932226 & 3.0223304 & 0.81\end{array}$ $\begin{array}{llll}95.09831643 & 2.397710827 & 0.77\end{array}$ $\begin{array}{llll}51.65524141 & 2.553341478 & 0.86\end{array}$ $\begin{array}{llll}56.94746884 & 2.100819984 & 0.85 \\ 60.15710578 & 2.070887511 & 0.94\end{array}$ $\begin{array}{llll}60.15710578 & 2.070887511 & 0.94\end{array}$ $\begin{array}{lllll}53.36318895 & 2.278573132 & 0.82\end{array}$ $\begin{array}{llll}51.00400982 & 1.901800465 & 0.85\end{array}$ $\begin{array}{llll}57.27151286 & 1.72380536 & 0.89\end{array}$ $\begin{array}{lllll}54.12991462 & 1.743910813 & 0.88\end{array}$ $\begin{array}{llll}55.25377642 & 1.736252492 & 0.86\end{array}$ $\begin{array}{lll}78.46715899 & 2.410014799 & 0.68\end{array}$ $\begin{array}{llll}75.29512694 & 2.181623784 & 0.91\end{array}$ $\begin{array}{lll}75.95617113 & 2.078734382 & 0.83\end{array}$ $\begin{array}{llll}71.79530684 & 1.625235026 & 0.84\end{array}$ $\begin{array}{llll}81.47599916 & 2.206565182 & 0.83 \\ 60.0181017 & 2.175058897 & 0.85\end{array}$ $\begin{array}{llll}72.97842354 & 1.177111484 & 0.85\end{array}$ $83.72437513 \quad 2.332625952 \quad 0.81$ $\begin{array}{llll}83.01239038 & 1.90735342 & 0.86\end{array}$ $\begin{array}{llll}133.2230587 & 1.653531686 & 0.89\end{array}$ $\begin{array}{llll}104.5184878 & 1.679055705 & 0.88\end{array}$ $\begin{array}{llll}132.1078669 & 1.487908603 & 1.00\end{array}$ $\begin{array}{lll}116.9957418 & 1.518089651 & 0.86\end{array}$ $\begin{array}{llll}98.10093987 & 1.399892029 & 0.93\end{array}$ $\begin{array}{llll}17.5491954 & 1.723535704 & 0.86\end{array}$ $\begin{array}{llll}116.6169576 & 1.578064794 & 0.88\end{array}$ $\begin{array}{llll}160.3493432 & 1.015625604 & 0.91\end{array}$ $\begin{array}{llll}171.5128032 & 1.177007586 & 0.92\end{array}$ $\begin{array}{llll}167.9816637 & 1.534050251 & 0.92\end{array}$ $\begin{array}{llll}172.2903995 & 1.881844725 & 0.89\end{array}$ $\begin{array}{llll}44.3718643 & 1.792067425 & 0.85\end{array}$ $\begin{array}{llll}68.7087503 & 1.332311193 & 0.88\end{array}$ $\begin{array}{lll}170.1573212 & 1.352431855 & 0.92\end{array}$ $\begin{array}{llll}170.8169097 & 1.054770823 & 0.87\end{array}$ $\begin{array}{llll}177.5453326 & 1.125802624 & 0.91\end{array}$ $\begin{array}{llll}213.7303432 & 1.204592704 & 0.90\end{array}$ $\begin{array}{llll}200.4371824 & 1.170119464 & 0.92\end{array}$ $\begin{array}{llll}216.7835004 & 1.188092332 & 0.98\end{array}$ $202.8914529 \quad 1.166807747 \quad 0.91$ $\begin{array}{llll}0.478755739 & 66.86297563 & 0.64\end{array}$ $\begin{array}{llll}75.66948797 & 3.823750308 & 0.64\end{array}$ $\begin{array}{llll}8.63132164 & 3.611625199 & 0.64\end{array}$ $\begin{array}{llll}174.0060101 & 1.946668496 & 0.64\end{array}$ $\begin{array}{llll}1.331234146 & 20.80612688 & 0.79\end{array}$ $\begin{array}{llll}121.1859302 & 3.586849607 & 0.79\end{array}$ $\begin{array}{llll}188.6371062 & 1.749029453 & 0.79 \\ 291.5492185 & 1.668334721 & 0.79\end{array}$ $\begin{array}{llll}0.171094142 & 371.0874043 \quad 0.76\end{array}$ $\begin{array}{llll}0.1708209793 & 250.5787239 & 0.26\end{array}$ $\begin{array}{llll}0.262978001 & 157.0752123 & 0.26\end{array}$ $\begin{array}{llll}0.193603492 & 295.1485413 & 0.35\end{array}$ $\begin{array}{lll}0.219878244 & 228.8244856 & 0.35\end{array}$ $\begin{array}{llll}0.294553845 & 127.5080557 & 0.35\end{array}$ $\begin{array}{llll}0.126881882 & 564.6237959 & 0.23\end{array}$ $\begin{array}{llll}0.150364495 & 402.0386483 & 0.23\end{array}$ $\begin{array}{llll}0.199223474 & 229.0220952 & 0.23\end{array}$ $\begin{array}{llll}0.093916857 & 765.0930985 & 0.24\end{array}$ $\begin{array}{llll}0.11331884 & 525.5295554 & 0.24\end{array}$ $\begin{array}{llll}0.135952303 & 402.1175318 & 0.30\end{array}$ $\begin{array}{llll}0.168159676 & 262.8345317 & 0.30\end{array}$ $\begin{array}{llll}0.203463909 & 179.5359316 & 0.30\end{array}$ $\begin{array}{llll}0.275412195 & 175.6862639 & 0.41\end{array}$ $\begin{array}{llll}0.317612431 & 132.1019275 & 0.41\end{array}$ $\begin{array}{llll}0.406455019 & 80.6638892 & 0.4\end{array}$ $\begin{array}{llll}0.208839843 & 203.7563895 & 0.46\end{array}$ $\begin{array}{llll}0.242535623 & 151.0730096 & 0.46 \\ 0.29437528 & 102.5499091 & 0.46\end{array}$ $\begin{array}{llll}0.29437528 & 102.5499091 & 0.46\end{array}$ 
$\begin{array}{llll}0.129592508 & 464.1423404 & 0.23\end{array}$ $\begin{array}{llll}0.171490829 & 265.050836 & 0.23 \\ 0.003019666 & 34197.41251 & 0.03\end{array}$ $\begin{array}{llll}0.003019666 & 34197.41251 & 0.03 \\ 0.00333577 & 33150.64545 & 0.03\end{array}$ $\begin{array}{llll}0.005580998 & 18182.27941 & 0.05\end{array}$ $\begin{array}{lllll}0.006764948 & 14160.03623 & 0.05\end{array}$ $\begin{array}{llll}0.011726545 & 7174.810976 & 0.06\end{array}$ $\begin{array}{llll}0.021096381 & 3567.331726 & 0.09\end{array}$ $\begin{array}{llll}0.004196008 & 34101.85505 & 0.03\end{array}$ $\begin{array}{llll}0.005137638 & 26908.94032 & 0.03 \\ 0.009221885 & 12822.48615 & 0.04\end{array}$ $\begin{array}{llll}0.009221885 & 12822.48615 & 0.04 \\ 0.010170024 & 11033.84554 & 0.04\end{array}$ $0.017791797 \quad 5488.940796 \quad 0.05$ $\begin{array}{llll}0.017791797 & 5488.940796 & 0.05 \\ 0.033042276 & 2560.926096 & 0.07\end{array}$ $\begin{array}{llll}0.007540234 & 23610.67276 \quad 0.02\end{array}$ $\begin{array}{llll}0.009430164 & 17857.16305 & 0.02\end{array}$ $\begin{array}{llll}0.017059573 & 8377.273726 & 0.03\end{array}$ $\begin{array}{llll}0.020601254 & 6516.394342 & 0.03\end{array}$ $\begin{array}{llll}0.036221598 & 3209.347446 & 0.04\end{array}$ $\begin{array}{lllll}0.065180528 & 1594.868887 & 0.06\end{array}$ $\begin{array}{llll}0.002531322 & 48664.93466 & 0.03 \\ 0.002620285 & 53726.31379 & 0.03\end{array}$ $\begin{array}{llll}0.002620285 & 53726.31379 & 0.03 \\ 0.003941136 & 36461.05931 & 0.05\end{array}$ $\begin{array}{llll}0.003941136 & 36461.05931 & 0.05 \\ 0.004479338 & 32297.26448 & 0.05\end{array}$ $0.007366252 \quad 18182.66109 \quad 0.06$ $\begin{array}{llll}0.007367252 & 18182.66109 & 0.06\end{array}$ $\begin{array}{lllll}0.002759791 & 78831.24572 & 0.03\end{array}$ $\begin{array}{lllll}0.003335742 & 63832.04363 & 0.03\end{array}$ $\begin{array}{llll}0.005600474 & 34766.58654 & 0.04\end{array}$ $\begin{array}{llll}0.007000953 & 23283.94065 & 0.04\end{array}$ $\begin{array}{lll}0.011199301 & 13853.0805 & 0.05 \\ 0.020569977 & 6607.988965 & 0.07\end{array}$ $\begin{array}{llll}0.020569977 & 6607.988965 & 0.07\end{array}$ $\begin{array}{llll}0.00440335 & 69232.74448 & 0.02 \\ 0.005477702 & 52924.18177 & 0.02\end{array}$ $\begin{array}{llll}0.005477702 & 52924.18177 & 0.02 \\ 0.009958861 & 24582.14103 & 0.03\end{array}$ $\begin{array}{llll}0.012211265 & 18546.98712 & 0.03\end{array}$ $\begin{array}{llll}0.012128153 & 9297.073737 & 0.04\end{array}$ $\begin{array}{llll}0.039675104 & 4304.518522 & 0.06\end{array}$ $\begin{array}{llll}0.105506498 & 1760.423019 & 0.03\end{array}$ $\begin{array}{lll}0.13067123 & 1357.652039 & 0.03\end{array}$ $\begin{array}{lll}0.221883 & 722.9169453 & 0.05\end{array}$ $\begin{array}{llll}0.25285435 & 620.2419654 & 0.05\end{array}$ $\begin{array}{llll}0.404513473 & 368.9719282 & 0.06\end{array}$ $\begin{array}{llll}0.71015219 & 192.6483217 & 0.09\end{array}$ $\begin{array}{llll}0.169870798 & 1309.913171 & 0.03 \\ 0.199515774 & 1123.307017 & 0.03\end{array}$ $\begin{array}{llll}0.327133212 & 641.4926695 & 0.04\end{array}$ 0.376147852540 .61655920 .04 $\begin{array}{llll}0.621445249 & 301.5491266 & 0.05\end{array}$ $\begin{array}{llll}0.62144189 & 170.007035 & 0.07\end{array}$ $\begin{array}{llll}0.270463003 & 1197.684046 & 0.02\end{array}$ $\begin{array}{llll}0.328827902 & 958.5033911 & 0.02\end{array}$ $\begin{array}{llll}0.554206651 & 518.0546059 & 0.03\end{array}$ $\begin{array}{llll}0.630103173 & 446.5408709 & 0.03\end{array}$ $\begin{array}{llll}1.004904686 & 267.295783 & 0.04\end{array}$ $\begin{array}{llll}1.672278292 & 155.3221984 & 0.06 \\ 0.899892097 & 285.0259198 & 0.03\end{array}$ $\begin{array}{llll}0.89982097 & 285.0259198 & 0.03 \\ 1.057480286 & 244.1707592 & 0.03\end{array}$ $\begin{array}{llll}1.617116906 & 160.3036481 & 0.05\end{array}$ $\begin{array}{llll}1.845591097 & 137.1263167 & 0.05\end{array}$ $2.81174071 \quad 89.94945724 \quad 0.06$ $\begin{array}{lllll}2.579582354 & 54.56399011 & 0.09\end{array}$ $\begin{array}{llll}1.205471306 & 287.2663092 & 0.03\end{array}$ $\begin{array}{llll}1.450197084 & 234.8104259 & 0.03\end{array}$ $\begin{array}{llll}2.229482777 & 152.528576 & 0.04\end{array}$ $\begin{array}{llll}2.522738651 & 132.7334796 & 0.04\end{array}$ $\begin{array}{llll}3.716360413 & 93.12079019 & 0.05\end{array}$ $\begin{array}{llll}1.943765217 & 2981188164 \quad 0.02\end{array}$ $\begin{array}{llll}1.9437652176 & 298.1188164 & 0.02\end{array}$ $\begin{array}{llll}2.214856283 & 166.5889877 & 0.03\end{array}$ $\begin{array}{llll}3.888716656 & 150.7269645 & 0.03\end{array}$ $\begin{array}{llll}5.856906228 & 101.1636087 & 0.04\end{array}$ $\begin{array}{llll}9.243141879 & 65.3627625 & 0.06\end{array}$ $\begin{array}{llll}93169.26169 & 0.504875547 & 1.00\end{array}$ $\begin{array}{llll}119347.6574 & 0.498547328 & 1.00\end{array}$ $\begin{array}{llll}64424.48408 & 0.485076506 & 1.00\end{array}$ $\begin{array}{llll}42721.07832 & 0.507113254 & 1.00\end{array}$ $\begin{array}{llll}46231.02607 & 0.41762268 & 1.00\end{array}$ $\begin{array}{llll}45820.1375 & 0.58645525 & 1.00\end{array}$ $\begin{array}{llll}46936.30023 & 0.504851762 & 1.00\end{array}$ $\begin{array}{lll}6919.683622 & 0.417613948 & 1.00\end{array}$ $\begin{array}{llll}18452.39324 & 0.457391485 & 1.00\end{array}$ $\begin{array}{llll}18105.00504 & 0.381217974 & 1.00\end{array}$ $\begin{array}{llll}10553.27699 & 0.42566935 & 1.00\end{array}$ $\begin{array}{llll}19.4373331 & 3.267357316 & 1.00\end{array}$ $\begin{array}{llll}52.35498105 & 1.504594577 & 1.00\end{array}$ $\begin{array}{llll}46.96278596 & 1.652684019 & 1.00\end{array}$ $\begin{array}{llll}80.40497233 & 1.277038147 & 1.00\end{array}$ $\begin{array}{llll}187.1128526 & 0.8015859 & 1.00\end{array}$ $\begin{array}{llll}146.7390745 & 1.0389248696 & 1.00\end{array}$ $\begin{array}{llll}15.93339155 & 3.589393596 & 1.00\end{array}$ $\begin{array}{llll}30.97339243 & 2.12145494 \quad 1.00\end{array}$ $\begin{array}{llll}64.73485209 & 1.436815362 & 1.00\end{array}$ $\begin{array}{llll}62.17621728 & 1.531283161 & 1.00\end{array}$ $\begin{array}{llll}111.5618991 & 1.12854534 & 1.00\end{array}$ $\begin{array}{llll}253.8666271 & 0.762484149 & 1.00\end{array}$ $\begin{array}{llll}260.9465729 & 0.723382398 & 1.00\end{array}$ $\begin{array}{llll}184.9314364 & 0.871157806 & 1.00\end{array}$ $\begin{array}{llll}22.16761169 & 2.60254817 & 1.00\end{array}$ $\begin{array}{llll}41.41671966 & 1.755670483 & 1.00\end{array}$ $84.23874599 \quad 1.25286804 \quad 1.00$ $\begin{array}{llll}148.7491988 & 0.930554929 & 1.00\end{array}$ $\begin{array}{llll}336.8031348 & 0.645178895 & 1.00\end{array}$ $\begin{array}{llll}256.2908493 & 0.861258285 & 1.00\end{array}$ $\begin{array}{lll}328.7117682 & 0.674505209 & 1.00\end{array}$ $\begin{array}{llll}61.39261557 & 1.237589308 & 1.00\end{array}$ $\begin{array}{llll}103.5238404 & 1.019602577 & 1.00\end{array}$ $\begin{array}{lll}224.5490182 & 0.742843316 & 1.00\end{array}$ $\begin{array}{llll}783.8511395 & 0.410568388 & 1.00\end{array}$ $\begin{array}{llll}205.5826539 & 0.755698183 & 1.00\end{array}$ $140.3986442 \quad 0.904839676 \quad 1.00$ $\begin{array}{lll}228.1544833 & 0.821621494 & 1.00\end{array}$ $\begin{array}{llll}500.6836217 & 0.576680995 & 1.00\end{array}$ $\begin{array}{llll}446.2647853 & 0.616490623 & 1.00\end{array}$ $\begin{array}{llll}809.0750341 & 0.435578903 & 1.00\end{array}$ $\begin{array}{llll}1426.103363 & 0.498547328 & 1.00\end{array}$ $\begin{array}{llll}1085.467127 & 0.524674588 & 1.00\end{array}$ $\begin{array}{llll}486.3784739 & 0.527000049 & 1.00\end{array}$ 
$\begin{array}{lll}1175.950421 & 0.395962166 & 1.00\end{array}$ $\begin{array}{llll}1175.950421 & 0.395962166 & 1.00 \\ 1855.258096 & 0.400744421 & 1.00\end{array}$ $\begin{array}{llll}1855.258164 & 0.497169857 & 1.00\end{array}$ $\begin{array}{llll}5916.811827 & 0.40079295 & 1.00\end{array}$ $\begin{array}{llll}4623.285909 & 0.395980821 & 1.00\end{array}$ $\begin{array}{llll}12303.87885 & 1.912165295 & 0.4\end{array}$ $\begin{array}{llll}6091.327026 & 2.349669906 & 0.48\end{array}$ $\begin{array}{llll}3450.962641 & 3.004873214 & 0.47\end{array}$ $\begin{array}{llll}8370.532171 & 1.245124224 & 0.48\end{array}$ $\begin{array}{llll}6847.700415 & 1.867686336 & 0.48\end{array}$ $\begin{array}{lllll}8168.032601 & 6.577067473 & 0.48\end{array}$ $\begin{array}{llll}8168.832601 & 6.577067473 & 0.48 \\ 16430.2754 & 5.358286641 & 0.47\end{array}$ $\begin{array}{llll}7.301845122 & 12.60045453 & 0.47\end{array}$ $\begin{array}{llll}14.72556826 & 8.824920248 & 0.48\end{array}$ $\begin{array}{llll}31.53633438 & 5.813182707 & 0.47\end{array}$ $\begin{array}{llll}10.72431798 & 9.309500574 & 0.47\end{array}$ $\begin{array}{llll}11.42008166 & 10.19506282 & 0.47\end{array}$ $\begin{array}{llll}20.93682366 & 6.663239063 & 0.48\end{array}$ $\begin{array}{llll}45.99629337 & 4.602409239 & 0.47\end{array}$ $\begin{array}{llll}6.386514648 & 13.25052124 & 0.48 \\ 14.90692917 & 7.520039805 & 0.47\end{array}$ $\begin{array}{llll}15.94884824 & 7.059301641 & 0.47\end{array}$ $\begin{array}{lllll}8.48160595 & 10.72128277 & 0.48\end{array}$ $\begin{array}{llll}8.4816049 & 10.72128277 & 0.48 \\ 19.06824172 & 7.04083273 & 0.47\end{array}$ $\begin{array}{llll}15.5227627 & 7.813381513 & 0.47\end{array}$ $\begin{array}{llll}28.16338181 & 5.594292044 & 0.48\end{array}$ $\begin{array}{llll}62.49437129 & 3.773085863 & 0.47\end{array}$ $\begin{array}{llll}60.43305415 & 3.999701332 & 0.47\end{array}$ $\begin{array}{llll}12.3717711 & 7.66383423 & 0.47\end{array}$ $\begin{array}{llll}21.97020184 & 5.971609802 & 0.48\end{array}$ $\begin{array}{lll}43.8662181 & 4.1169765 & 0.47\end{array}$ $\begin{array}{lllll}64.54386252 & 3.956281808 & 0.48\end{array}$ $\begin{array}{llll}125.2307576 & 3.413427046 & 0.47\end{array}$ $\begin{array}{llll}125.2307576 & 3.413427046 & 0.47\end{array}$ $\begin{array}{llll}126.2326037 & 3.353890527 & 0.47\end{array}$ $\begin{array}{llll}142.2621406 & 2.659297815 & 0.47\end{array}$ $\begin{array}{llll}122.2252194 & 3.542422835 & 0.47\end{array}$ $\begin{array}{llll}130.2399879 & 3.175280973 & 0.47\end{array}$ $\begin{array}{llll}114.2104509 & 4.108019758 & 0.47\end{array}$ $\begin{array}{llll}114.2104509 & 4.127865264 & 0.47\end{array}$ $\begin{array}{llll}123.2270655 & 3.49280907 & 0.47\end{array}$ $\begin{array}{lllll}128.2362958 & 3.234817491 & 0.47\end{array}$ $\begin{array}{llll}2603.182802 & 3.482468956 & 0.47\end{array}$ $\begin{array}{lllll}2603.182802 & 3.942468956 & 0.47\end{array}$ $\begin{array}{lllll}5536.823164 & 1.867686336 & 0.48\end{array}$ $\begin{array}{lllll}7902.491296 & 3.021815621 & 0.47\end{array}$ $\begin{array}{llll}10803.40582 & 1.634752713 & 0.47\end{array}$ $\begin{array}{llll}2870.030434 & 14.66604941 & 0.47\end{array}$ $\begin{array}{llll}4485.676937 & 6.046616959 & 0.4\end{array}$ $\begin{array}{llll}6353.536467 & 7.27996018 & 0.48\end{array}$ $\begin{array}{llll}17732.67527 & 3.085976195 & 0.47\end{array}$ $\begin{array}{lllll}12422.89115 & 6.310870933 & 0.47\end{array}$ $\begin{array}{llll}27.62021233 & 5.164326275 & 0.47\end{array}$ $\begin{array}{llll}94.02964327 & 4.022482434 & 0.47\end{array}$ $\begin{array}{llll}94.026303585 & 4.464886033 & 0.47\end{array}$ $\begin{array}{lllll}116.9857508 & 4.568802596 & 0.48\end{array}$ $\begin{array}{lllll}97.03058933 & 3.82433059 & 0.47\end{array}$ $\begin{array}{llll}72.25251442 & 5.145630989 & 0.47\end{array}$ $\begin{array}{llll}129.087725 & 3.735372672 & 0.48\end{array}$ $\begin{array}{llll}173.3193685 & 6.797085832 & 0.47\end{array}$ $\begin{array}{llll}212.3913649 & 4.524775386 & 0.47\end{array}$ $\begin{array}{llll}99.03122003 & 3.675716707 & 0.47\end{array}$ $\begin{array}{llll}76.266543 & 4.444864122 & 0.47 \\ 129.087725 & 3.735372672 & 0.48\end{array}$ $\begin{array}{lllll}110.7100732 & 5.472451217 & 0.47\end{array}$ $\begin{array}{lllll}120.6839536 & 4.666508401 & 0.47\end{array}$ $\begin{array}{lllll}252.8838005 & 5.886441676 & 0.47\end{array}$ $\begin{array}{llll}245.8592505 & 6.186770333 & 0.47\end{array}$ $\begin{array}{llll}182.5381112 & 5.392191195 & 0.48\end{array}$ $\begin{array}{llll}397.3481537 & 5.834009468 & 0.48\end{array}$ $\begin{array}{llll}310.0977597 & 5.696865516 & 0.47\end{array}$ $\begin{array}{llll}38.3605468 & 5.655969233 & 0.47\end{array}$ $\begin{array}{llll}11897.05115 & 1.27138508 & 0.74\end{array}$ $\begin{array}{llll}6368.516044 & 1.349430714 & 0.74\end{array}$ $\begin{array}{llll}32638.40334 & 0.845063011 & 0.73\end{array}$ $\begin{array}{llll}32638.40334 & 0.845063011 & 0.73 \\ 18156.13743 & 0.805555713 & 0.73\end{array}$ $\begin{array}{llll}14.47356681 & 5.417207945 \quad 0.73\end{array}$ $\begin{array}{lllll}33.35825814 & 3.282985994 & 0.73\end{array}$ $\begin{array}{llll}9.651898107 & 6.945719665 & 0.74\end{array}$ $\begin{array}{llll}10.47062466 & 6.983352704 & 0.73\end{array}$ $\begin{array}{llll}19.57499626 & 4.680823371 & 0.73\end{array}$ $\begin{array}{llll}41.58193949 & 3.448854537 & 0.73\end{array}$ \begin{tabular}{llll}
13.8481309 & 5.524247515 & 0.74 \\
\hline
\end{tabular} $\begin{array}{llll}60.06104978 & 2.444098333 & 0.73\end{array}$ $\begin{array}{llll}26.70061597 & 3.716327944 & 0.73 \\ 19.06531223 & 4.617033022 & 0.74\end{array}$ $\begin{array}{lllll}15.649186 & 5.128826476 & 0.73\end{array}$ $\begin{array}{lllll}10.42698167 & 6.533951124 & 0.73\end{array}$ $\begin{array}{llll}19.07602241 & 4.652384338 & 0.74\end{array}$ $\begin{array}{llll}48.08858884 & 2.607219893 & 0.74\end{array}$ $\begin{array}{lll}37.31166785 & 3.09077024 & 0.73\end{array}$ $\begin{array}{llll}63.84575798 & 2.426612272 & 0.73\end{array}$ $\begin{array}{llll}146.1719904 & 1.570823008 & 0.73\end{array}$ $\begin{array}{llll}13966.25956 & 0.895061904 & 0.73\end{array}$ $\begin{array}{llll}24428.7436 & 1.004133695 & 0.73 \\ 10082.24673 & 1.181286924 & 0.74\end{array}$ $\begin{array}{llll}10082.24673 & 1.181286924 & 0.74\end{array}$ $\begin{array}{llll}25.86905372 & 4.081317385 & 0.73 \\ 47.29044187 & 3.0860827 & 0.74\end{array}$ $\begin{array}{llll}110.9047141 & 1.89206126 & 0.74\end{array}$ $\begin{array}{llll}84.33981284 & 2.292403915 & 0.73\end{array}$ $\begin{array}{llll}151.6336752 & 1.611111426 & 0.73\end{array}$ $\begin{array}{llll}308.3628291 & 1.322275064 & 0.73\end{array}$ $\begin{array}{lllll}151.8440378 & 1.768455211 & 0.73\end{array}$ $\begin{array}{llll}458.2801879 & 1.156345338 & 0.73\end{array}$ $\begin{array}{llll}262.5346388 & 1.523221033 & 0.74\end{array}$ $\begin{array}{llll}719.2623673 & 1.064129152 & 0.73\end{array}$ $\begin{array}{llll}530.3261782 & 1.241352361 & 0.74\end{array}$ $\begin{array}{llll}32391.3002 & 0.938458626 & 0.92\end{array}$ $\begin{array}{llll}32213.40404 & 0.852190422 & 0.92\end{array}$ $\begin{array}{llll}28966.23824 & 1.168285229 & 0.92\end{array}$ $\begin{array}{lllll}21798.66729 & 0.595974791 & 0.92\end{array}$ $\begin{array}{lllll}40645.41366 & 0.834262122 & 0.93\end{array}$ $\begin{array}{llll}27986.13089 & 0.849453268 & 0.93\end{array}$ $\begin{array}{llll}53.04443497 & 2.021000626 & 0.93\end{array}$ $\begin{array}{llll}115.8265533 & 1.51124006 & 0.91\end{array}$ $\begin{array}{llll}115.72765523 & 3.985922737 & 0.92\end{array}$ 

$\begin{array}{llll}43.66311601 & 2.438007076 & 0.93 \\ 68.55852906 & 1.998348805 & 0.93\end{array}$ $\begin{array}{llll}150.4755223 & 1.462802879 & 0.91\end{array}$ $\begin{array}{lll}13.446613 & 4.494267152 & 0.92\end{array}$ $\begin{array}{llll}23.91543407 & 3.0243425 & 0.92\end{array}$ $\begin{array}{llll}57.09447584 & 1.838149283 & 0.92\end{array}$ $\begin{array}{llll}17.5107639 & 3.699007268 & 0.92\end{array}$ $\begin{array}{llll}32.06989651 & 2.486907864 & 0.92\end{array}$ $\begin{array}{llll}67.52264996 & 1.896069469 & 0.92\end{array}$ $\begin{array}{lllll}60.88210931 & 1.796544842 & 0.93\end{array}$ $\begin{array}{llll}194.0342261 & 1.507803898 & 0.91\end{array}$ $\begin{array}{llll}47.015261 & 1.302803898 & 0.91\end{array}$ $\begin{array}{llll}47.80153333 & 1.828153304 & 0.92\end{array}$ $\begin{array}{llll}155.5956716 & 1.311926855 & 0.92\end{array}$ $\begin{array}{llll}145.3314867 & 1.152132018 & 0.93\end{array}$ $\begin{array}{lll}213.5108476 & 1.125283793 & 0.93\end{array}$ $\begin{array}{llll}420.73748 & 1.026868246 & 0.91\end{array}$ $\begin{array}{llll}105.3898575 & 1.439907954 & 0.92\end{array}$ $\begin{array}{llll}172.6138812 & 1.201719661 & 0.92\end{array}$ $\begin{array}{llll}346.420552 & 1.024643602 & 0.92\end{array}$ $\begin{array}{lll}308.3384246 & 0.966619236 & 0.93 \\ 445.6304389 & 0.979772958 & 0.93\end{array}$ $\begin{array}{llll}847.4147833 & 0.949368756 & 0.91\end{array}$ $\begin{array}{llll}500.355585 & 0.969733928 & 0.92\end{array}$ $\begin{array}{llll}1306.019442 & 0.781106453 & 0.93\end{array}$ $\begin{array}{llll}796.9829488 & 0.908617305 & 0.92\end{array}$ $\begin{array}{llll}1851.080285 & 0.853663567 & 0.93\end{array}$ $\begin{array}{llll}3306.501608 & 0.939681319 & 0.91\end{array}$ $\begin{array}{lll}1516.813151 & 0.823545325 & 0.92\end{array}$ $\begin{array}{llll}24475.128 & 0.768976996 & 0.93\end{array}$ $\begin{array}{llll}9355.640473 & 0.654736941 & 0.94 \\ 14486.0615 & 0.665390548 & 0.93\end{array}$ $\begin{array}{llll}14486.0615 & 0.665390548 & 0.93\end{array}$ $\begin{array}{llll}2577.80826 & 0.6995624 & 0.93\end{array}$ $\begin{array}{lllll}33813.76244 & 0.635077706 & 0.93\end{array}$ $\begin{array}{llll}538185.38544 & 0.63507706 & 0.93\end{array}$ $\begin{array}{llll}34600.12901 & 0.654618558 & 0.93\end{array}$ $\begin{array}{llll}20.28549092 & 3.190529419 & 0.93\end{array}$ $\begin{array}{lll}37.70512115 & 2.054446453 & 0.93\end{array}$ $\begin{array}{lll}77.98007106 & 1.696058636 & 0.93\end{array}$ $\begin{array}{llll}23.39679786 & 2.940260003 & 0.93\end{array}$ $\begin{array}{llll}27.29752239 & 2.751063625 & 0.93\end{array}$ $\begin{array}{llll}50.79233864 & 1.902788617 & 0.93 \\ 110.9716396 & 1.360791231 & 0.93\end{array}$ $\begin{array}{llll}11.9716396 & 1.360791231 & 0.93 \\ 14.69340448 & 3.950410194 & 0.93\end{array}$ $\begin{array}{llll}14.69173243 & 1.897928856 & 0.93\end{array}$ $\begin{array}{llll}148.9619306 & 1.124131886 & 0.93\end{array}$ $\begin{array}{llll}66.84115832 & 1.582809052 & 0.93\end{array}$ $\begin{array}{llll}36.29849058 & 2.395940417 & 0.93\end{array}$ $\begin{array}{llll}19.84509349 & 3.180060756 & 0.93\end{array}$ $\begin{array}{llll}46.19182713 & 1.963967385 & 0.93\end{array}$ $\begin{array}{llll}27.67729131 & 2.450168208 & 0.94\end{array}$ $\begin{array}{llll}50.58002644 & 1.786083482 & 0.93\end{array}$ $\begin{array}{llll}110.8873146 & 1.242193609 & 0.93\end{array}$ $\begin{array}{llll}94.16341602 & 1.290328728 & 0.93\end{array}$ $\begin{array}{llll}162.1881048 & 1.006353903 & 0.93\end{array}$ $\begin{array}{llll}67.67910129 & 1.58724107 & 0.94\end{array}$ $\begin{array}{llll}121.8827244 & 1.181813062 & 0.93\end{array}$ $\begin{array}{llll}248.7472192 & 0.946433226 & 0.93\end{array}$ $\begin{array}{llll}209.9059482 & 0.977521764 & 0.93\end{array}$ $\begin{array}{llll}342.0694573 & 0.85979751 & 0.93\end{array}$ $\begin{array}{llll}581.8512994 & 0.749421258 & 0.93\end{array}$ $\begin{array}{lll}338.3955065 & 0.476172321 & 0.94\end{array}$ $\begin{array}{llll}1000.486295 & 0.625613929 & 0.93\end{array}$ $\begin{array}{llll}59.4232346 & 0.794496176 & 0.93 \\ 1612.051465 & 0.57645148 & 0.93\end{array}$ $\begin{array}{llll}1198.781779 & 0.630955484 & 0.93\end{array}$ $\begin{array}{llll}3119202842 & 0.532483525 & 0.93\end{array}$ $\begin{array}{llll}3.545781019 & 9.002097905 & 0.94\end{array}$ $\begin{array}{llll}4.100538721 & 7.995932518 & 0.94\end{array}$ $\begin{array}{llll}4.919210024 & 6.893589932 & 0.94\end{array}$ $\begin{array}{llll}6.56732503 & 5.598891648 & 0.94\end{array}$ $\begin{array}{llll}3.59425502 & 9.823385891 & 0.80\end{array}$ $\begin{array}{llll}4.154398422 & 8.805115905 & 0.80\end{array}$ $\begin{array}{llll}4.989228026 & 7.67965892 & 0.80\end{array}$ $\begin{array}{llll}6.658887032 & 6.24604124 & 0.80\end{array}$ $\begin{array}{llll}3.599640825 & 10.96224085 & 0.90 \\ 4.149012628 & 9.850182865 & 0.90\end{array}$ $4.989228033 \quad 8.630937886 \quad 0.90$ $\begin{array}{llll}6.648114741 & 7.049938913 & 0.90 \\ 3.56193903 & 12.82460379 & 0.70\end{array}$ $\begin{array}{llll}3.56193903 & 12.82460379 & 0.70\end{array}$ $\begin{array}{lllll}4.105924633 & 11.56516481 & 0.70\end{array}$ $\begin{array}{llll}4.940754039 & 10.15834284 & 0.70\end{array}$ $\begin{array}{llll}6.588869048 & 8.336174878 & 0.70\end{array}$ $\begin{array}{lll}0.354590051 & 86.67942443 & 0.43\end{array}$ $\begin{array}{lll}2.491362916 & 14.874886 & 0.43\end{array}$ $\begin{array}{llll}11.68884936 & 4.742683799 & 0.43\end{array}$ $\begin{array}{llll}11.68884936 & 4.742683799 & 0.43\end{array}$ $\begin{array}{llll}34.73172541 & 2.316362461 & 0.43 \\ 53.73695123 & 1.86827359 & 0.43\end{array}$ $\begin{array}{llll}91.74401695 & 1.380442309 & 0.43\end{array}$ $\begin{array}{lllll}105.8782009 & 1.320080486 & 0.43\end{array}$ $\begin{array}{llll}315.0167327 & 0.950174873 & 0.43\end{array}$ $\begin{array}{llll}552.1156649 & 0.800371886 & 0.43\end{array}$ $\begin{array}{llll}912.9605837 & 0.676259935 & 0.43\end{array}$ $\begin{array}{llll}1346.230168 & 0.679884647 & 0.43\end{array}$ $\begin{array}{llll}1909.122891 & 0.846007557 & 0.43\end{array}$ $\begin{array}{llll}0.338009599 & 108.6017415 & 0.4\end{array}$ $\begin{array}{llll}2.256479258 & 20.28698763 & 0.41 \\ 8.440026625 & 7.078798468 & 0.41\end{array}$ $\begin{array}{llll}8.440026625 & 7.078798468 & 0.41\end{array}$ $\begin{array}{llll}34.46810831 & 4.314187844 & 0.41\end{array}$ $\begin{array}{llll}84.88219961 & 2.160551889 & 0.41\end{array}$ $\begin{array}{llll}273.5179199 & 1.49211656 & 0.41\end{array}$ $\begin{array}{lll}290.1952676 & 1.326519315 & 0.4\end{array}$ $\begin{array}{llll}463.7951832 & 1.343528857 & 0.41\end{array}$ $\begin{array}{llll}767.9264971 & 1.3484343465 & 0.41\end{array}$ $\begin{array}{llll}1154.222094 & 1.147330198 & 0.41\end{array}$ $\begin{array}{llll}1762.670934 & 1.174417579 & 0.41 \\ 0.17054012 & 180.2219857 & 0.52\end{array}$ $\begin{array}{llll}17.170545012 & 1.180 .2219857 & 0.52 \\ 1.359741441 & 27.91920783 & 0.52\end{array}$ $\begin{array}{llll}1.359741441 & 27.91920783 & 0.52\end{array}$ $\begin{array}{lllll}21.1481132 & 4.574646766 & 0.52\end{array}$ $\begin{array}{lllll}21.148132 & 4.574646766 & 0.52\end{array}$ $\begin{array}{llll}52.0338409 & 2.934544356 & 0.52\end{array}$ $\begin{array}{llll}57.90641031 & 2.771448325 & 0.52\end{array}$ $\begin{array}{llll}159.2888306 & 1.815914095 & 0.52\end{array}$ $\begin{array}{lll}220.5731035 & 1.529487855 & 0.52\end{array}$ $\begin{array}{llll}349.3511369 & 1.393269451 & 0.52\end{array}$ $\begin{array}{llll}562.1908135 & 1.062313196 & 0.52\end{array}$ $\begin{array}{lllll}876.3164754 & 0.944402072 & 0.52\end{array}$

$\begin{array}{lllll}1324.661894 & 0.853063426 & 0.52\end{array}$ 
$\begin{array}{llll}0.13790864 & 240.2285165 & 0.52\end{array}$ $\begin{array}{llll}1.246247647 & 34.44369855 & 0.52 \\ 4.505383213 & 13.37018397 & 0.52\end{array}$ $\begin{array}{llll}8.847355329 & 8.169205271 & 0.52\end{array}$ $\begin{array}{llll}15.36607792 & 6.539119375 & 0.52\end{array}$ $\begin{array}{llll}16.39616755 & 6.241411807 & 0.52\end{array}$ $\begin{array}{llll}30.52350507 & 4.541681192 & 0.52\end{array}$ $\begin{array}{llll}36.49899844 & 4.102249417 & 0.52\end{array}$ $\begin{array}{lll}106.6386829 & 2.870621868 & 0.52 \\ 113.9106943 & 2.71964201 & 0.52\end{array}$ $\begin{array}{llll}113.9106943 & 2.719648201 & 0.52 \\ 151.5835303 & 2.440729247 & 0.52\end{array}$ $\begin{array}{llll}151.5835303 & 2.440729247 & 0.52\end{array}$ $407.6000901 \quad 1.933258177 \quad 0.52$ $612.638099 \quad 1.755645187 \quad 0.52$ $\begin{array}{llll}962.5806899 & 1.595958323 & 0.52\end{array}$ $\begin{array}{llll}0.163603409 & 224.0603755 & 0.52\end{array}$ $\begin{array}{llll}1.414234649 & 31.7538236 & 0.52\end{array}$ $\begin{array}{llll}1.493664433 & 30.63070809 & 0.5\end{array}$ $\begin{array}{lll}4.929588561 & 12.36635946 & 0.52\end{array}$ $\begin{array}{llll}9.404071085 & 8.294049866 & 0.52\end{array}$ $\begin{array}{llll}15.81122826 & 6.7135328 & 0.52\end{array}$ $\begin{array}{llll}16.81366399 & 6.233483324 & 0.52 \\ 17.90914389 & 6.138578395 & 0.52\end{array}$ $\begin{array}{llll}17.90914389 & 6.138578395 & 0.52 \\ 38.50450989 & 4.220814385 & 0.52\end{array}$ $45.46720069-4.165125396 \quad 0.52$ $\begin{array}{llll}45.46720069 & 4.165125396 & 0.52 \\ 104.8388771 & 3.011768745 & 0.52\end{array}$ $\begin{array}{llll}114.0502487 & 2.79647432 & 0.52\end{array}$ $\begin{array}{llll}162.8609751 & 2.512648353 & 0.52\end{array}$ $\begin{array}{llll}172.6002591 & 2.238610189 & 0.52\end{array}$ $\begin{array}{llll}247.3059699 & 2.134352399 & 0.52\end{array}$ $\begin{array}{llll}261.4973177 & 1.985953383 & 0.52\end{array}$ $\begin{array}{llll}443.694555 & 1.826491956 & 0.52 \\ 705.52353 & 1.743298387 & 0.52\end{array}$ $\begin{array}{llll}705.52353 & 1.743298387 & 0.52 \\ 846.427288 & 1.788227794 & 0.52\end{array}$ $\begin{array}{lll}846.427288 & 1.788227794 & 0.52 \\ 0.147313018 & 242.5245113 & 0.52\end{array}$ $\begin{array}{lllll}0.1440004579 & 31.54434236 & 0.52\end{array}$ $\begin{array}{llll}1.768519043 & 12.54846978 & 0.52\end{array}$ $\begin{array}{llll}8.968363291 & 9.375498385 & 0.52\end{array}$ $\begin{array}{llll}15.32994182 & 6.574182675 & 0.52\end{array}$ $\begin{array}{llll}17.15917715 & 6.248666903 & 0.52\end{array}$ $\begin{array}{llll}38.01987783 & 4.120090487 & 0.52\end{array}$ $\begin{array}{llll}43.88334887 & 4.248659005 & 0.52\end{array}$ $\begin{array}{lll}108.3443514 & 2.895073526 & 0.52\end{array}$ $\begin{array}{lllll}160.26688 & 2.425477904 & 0.52 \\ 248.6897375 & 2.191039815 & 0.52\end{array}$ $\begin{array}{llll}248.6897375 & 2.191039815 & 0.52 \\ 467.7217718 & 1.616446534 & 0.52\end{array}$ $\begin{array}{lllll}724.1017841 & 1.546137886 & 0.52\end{array}$ $\begin{array}{lllll}878.7128871 & 1.515340357 & 0.52\end{array}$ $\begin{array}{lllll}0.001000004 & 43823.31451 & 0.74\end{array}$ $\begin{array}{llll}0.010000036 & 4382.338451 & 0.74\end{array}$ $\begin{array}{llll}1.000003526 & 48.56865753 & 0.74\end{array}$ $\begin{array}{llll}10.00002377 & 6.938967008 & 0.74\end{array}$ $\begin{array}{lll}50.00007208 & 2.096993954 & 0.74\end{array}$ $\begin{array}{lll}100 & 1.264 & 0.74 \\ 0.001000003 & 29506.65349 & 0.93\end{array}$ $\begin{array}{llll}0.01000027 & 2950.674349 & 0.93 \\ 1.000002598 & 32.68683018 & 0.93\end{array}$ $\begin{array}{llll}1.00002598 & 32.68683018 & 0.93 \\ 10.00001752 & 4.767983297 & 0.93\end{array}$ $\begin{array}{llll}1 & 0.000005311 & 1.60499659 & 0.93\end{array}$ $\begin{array}{llll}100 & 1.119 & 0.93\end{array}$ $\begin{array}{lll}0.001000001 & 22313.02082 & 0.93\end{array}$ $\begin{array}{lll}0.010000013 & 2231.304082 & 0.93\end{array}$ $\begin{array}{llll}1.000001299 & 25.1759346 & 0.93\end{array}$ $\begin{array}{llll}10.00000876 & 4.331792412 & 0.93\end{array}$ $\begin{array}{llll}50.00002656 & 1.732798159 & 0.93\end{array}$ $\begin{array}{lll}100 & 1.1744 & 0.93 \\ 0.001000001 & 21260.33939 & 0.74\end{array}$ $\begin{array}{lll}0.001000001 & 21260.33939 & 0.74 \\ 0.010000007 & 2126.036939 & 0.74\end{array}$ $\begin{array}{llll}0.01000007 & 2126.036939 & 0.74 \\ 1.000000705 & 24.6799652 & 0.74\end{array}$ $\begin{array}{llll}10.00000475 & 4.665995563 & 0.74\end{array}$ $\begin{array}{llll}10.00001442 & 2.698998732 & 0.74\end{array}$ $\begin{array}{llll}100 & 1.607 & 0.74 \\ 1314.901047 & 0.87162991 & 0.84\end{array}$ $\begin{array}{llll}1314.901047 & 0.871628991 & 0.84 \\ 2389.549242 & 0.890757766 & 0.84\end{array}$ $\begin{array}{llll}3863.37981 & 0.807742593 & 0.84\end{array}$ $\begin{array}{llll}5556.118665 & 0.762772118 & 0.84\end{array}$ $\begin{array}{llll}7206.470479 & 0.783495217 & 0.84\end{array}$ $\begin{array}{llll}8837.942709 & 0.827217242 & 0.84\end{array}$ $\begin{array}{llll}162.6698023 & 2.06151781 & 0.84 \\ 332.4240373 & 1.666057195 & 0.84\end{array}$ $\begin{array}{lllll}534.3561587 & 1.528372861 & 0.84\end{array}$ $\begin{array}{lllll}534.3561587 & 1.528372861 & 0.84\end{array}$ $\begin{array}{llll}1187.68969 & 1.044140064 & 0.84\end{array}$ $\begin{array}{llll}1504.510494 & 1.033271996 & 0.84\end{array}$ $\begin{array}{llll}27.71570323 & 5.532473989 & 0.84\end{array}$ $\begin{array}{llll}61.04777561 & 3.848622143 & 0.84\end{array}$ $\begin{array}{llll}111.374559 & 2.74087467 & 0.84\end{array}$ $\begin{array}{llll}187.1239224 & 1.89641153 & 0.84\end{array}$ $\begin{array}{llll}262.0333441 & 1.671174618 & 0.84\end{array}$ $\begin{array}{llll}347.5043273 & 1.508880634 & 0.84\end{array}$ $\begin{array}{llll}929.0410985 & 1.746014942 & 0.84 \\ 1444.890134 & 2.436250464 & 0.84\end{array}$ $\begin{array}{lllll}2055.095365 & 2.854590634 & 0.84\end{array}$ $\begin{array}{lllll}3900.490158 & 1.547745187 & 0.84\end{array}$ $\begin{array}{llll}5301.367346 & 1.447790677 & 0.84\end{array}$ $\begin{array}{lllll}7021.330985 & 1.310638888 & 0.84\end{array}$ $\begin{array}{llll}151.9505628 & 2.362633418 & 0.84\end{array}$ $\begin{array}{llll}290.9083295 & 2.175516757 & 0.84\end{array}$ $\begin{array}{llll}431.9897043 & 2.338536555 & 0.84\end{array}$ $\begin{array}{llll}636.1263705 & 2.106367745 & 0.84\end{array}$ $\begin{array}{llll}816.471446 & 2.209442638 & 0.84\end{array}$ $\begin{array}{llll}29.2313129 & 2.429179729 & 0.84\end{array}$ $\begin{array}{llll}29.91093386 & 3.624037632 & 0.84\end{array}$ $\begin{array}{llll}111.1310477 & 2.752899488 & 0.84\end{array}$ $\begin{array}{llll}176.0367229 & 2.142814918 & 0.84\end{array}$ $\begin{array}{lllll}228.1729015 & 2.203975789 & 0.84\end{array}$ $\begin{array}{llll}281.4327952 & 2.300519455 & 0.84\end{array}$ $\begin{array}{llll}9038.244029 & 0.620983247 & 0.88\end{array}$ $\begin{array}{llll}9795.438634 & 0.632006414 & 0.8\end{array}$ $\begin{array}{llll}11283.44094 & 0.539314671 & 0.89\end{array}$ $\begin{array}{llll}12337.12532 & 0.494913669 & 0.88 \\ 13404.73318 & 0.4446082 & 0.84\end{array}$ $\begin{array}{llll}13404.73318 & 0.44466082 & 0.84 \\ 1465.769275 & 0.854672915 & 0.88\end{array}$ $\begin{array}{llll}1465.769275 & 0.854672915 & 0.88\end{array}$ $\begin{array}{lllll}168.859838 & 0.77694371 & 0.89\end{array}$ $\begin{array}{llll}2090.475881 & 0.623950645 & 0.88\end{array}$ $\begin{array}{llll}2191.586415 & 0.602158988 & 0.84\end{array}$ $\begin{array}{llll}322.418967 & 1.376132405 & 0.88\end{array}$ $\begin{array}{llll}368.5337151 & 1.259123091 & 0.89\end{array}$ $\begin{array}{llll}418.8762845 & 1.10358855 & 0.89\end{array}$ $\begin{array}{llll}465.3076996 & 0.981138459 & 0.88\end{array}$ $\begin{array}{lll}482.5719411 & 0.967552315 & 0.84 \\ 5476.942251 & 1.691109332 & 0.88\end{array}$ 


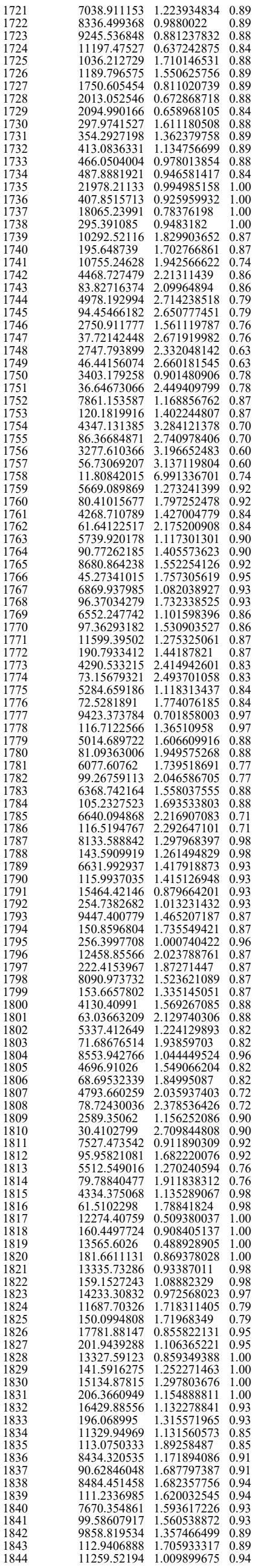




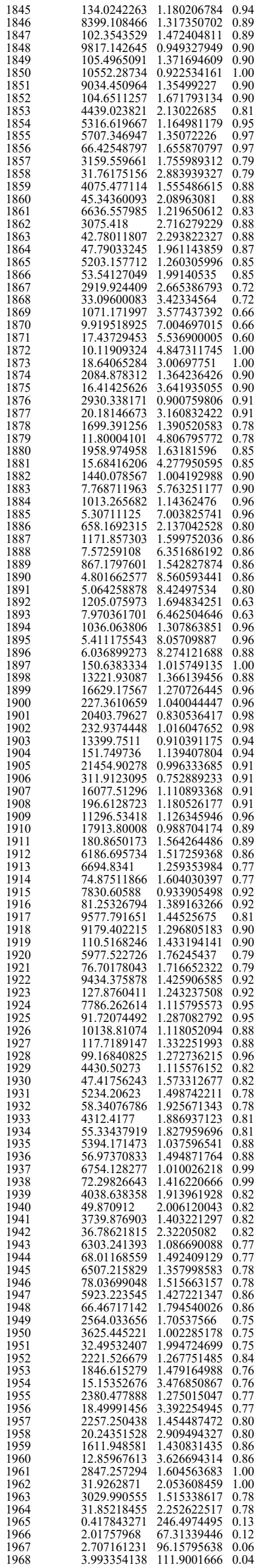


$\begin{array}{llll}40.56023951 & 9.314309278 & 0.29\end{array}$

$\begin{array}{llll}39.04698381 & 8.719612259 & 0.29\end{array}$ $\begin{array}{llll}37.29647351 & 10.42750501 & 0.28\end{array}$ $\begin{array}{llll}37.98747823 & 8.900563433 & 0.25 \\ 39.22179433 & 11.42193729 & 0.24\end{array}$ $\begin{array}{lll}39.22179433 & 11.42193729 & 0.24 \\ 37.62640919 & 12.00193973 & 0.24\end{array}$ $\begin{array}{llll}37.62640919 & 11.00193973 & 0.24\end{array}$ $\begin{array}{llll}30.32814682 & 11.29366504 & 0.24 \\ 30.7386023 & 14.29149705 & 0.19\end{array}$ $\begin{array}{lll}30.33866023 & 14.29149705 & 0.19 \\ 30.70081604 & 17.11193986 & 0.16\end{array}$ $\begin{array}{llll}28.68199666 & 18.57918552 & 0.13\end{array}$ $\begin{array}{llll}27.49493201 & 23.83314739 & 0.13\end{array}$ $\begin{array}{lll}26.04798428 & 22.54234251 & 0.12\end{array}$ $\begin{array}{llll}26.43652058 & 32.97233425 & 0.11\end{array}$ $\begin{array}{llll}27.67351218 & 36.20897967 & 0.10\end{array}$ $\begin{array}{llll}27.25161044 & 34.9535749 & 0.10\end{array}$ $\begin{array}{llll}26.0695885 & 34.94598466 & 0.10 \\ 18.0603589 & 93.2960645 & 0.03\end{array}$ $\begin{array}{llll}18.0603589 & 93.29640645 & 0.03 \\ 18.64288779 & 92.17672908 & 0.03\end{array}$ $\begin{array}{llll}18.64288779 & 92.17672908 & 0.03 \\ 18.98484405 & 107.6081565 & 0.03\end{array}$ $23.24175487 \quad 68.88500561 \quad 0.04$ $\begin{array}{llll}23.24175487 & 68.88500561 & 0.04 \\ 29.67126983 & 38.59800034 & 0.06\end{array}$ $\begin{array}{llll}25.67221465 & 25.78766029 & 0.08\end{array}$ $\begin{array}{llll}26.72732045 & 34.38698761 & 0.08\end{array}$ $\begin{array}{llll}27.06632685 & 33.31169736 & 0.09\end{array}$ $\begin{array}{llll}28.37631053 & 56.33535342 & 0.05\end{array}$ $\begin{array}{llll}26.97773512 & 51.94456653 & 0.05 \\ 1119.793877 & 11.34179887 & 0.39\end{array}$ $\begin{array}{lll}1119.793877 & 11.34179887 & 0.39\end{array}$ $\begin{array}{lll}280.747724 & 15.56169098 & 0.23 \\ 569.707294 & 22.43074953 & 0.25\end{array}$ $\begin{array}{llll}569.707294 & 22.43074953 & 0.25 \\ 3494.610293 & 5.652787376 & 0.26\end{array}$ $\begin{array}{llll}3494.610293 & 5.652787376 & 0.26 \\ 2674.677166 & 4.342401013 & 0.53\end{array}$ $\begin{array}{llll}214.0236368 & 5.245174894 & 0.36\end{array}$ $\begin{array}{llll}419.5529297 & 10.26877434 & 0.38\end{array}$ $\begin{array}{llll}710.9056613 & 7.600357347 & 0.37\end{array}$ $\begin{array}{llll}328.9226376 & 34.07894611 & 0.17\end{array}$ $\begin{array}{llll}2570.559324 & 11.21649104 & 0.33\end{array}$ $\begin{array}{llll}3084.740472 & 8.049506449 & 0.37\end{array}$ $\begin{array}{llll}598.5958352 & 10.8116416 & 0.37\end{array}$ $\begin{array}{llll}1401.336836 & 7.781951617 & 0.37\end{array}$ $\begin{array}{llll}143.0138005 & 11.98567252 & 0.37 \\ 1852.81228 & 13.11236075 & 0.37\end{array}$ $\begin{array}{llll}1252.841966 & 56.33579966 & 0.37\end{array}$ $\begin{array}{llll}1252.041966 & 56.33579966 & 0.11 \\ 1456.864866 & 64.08105699 & 0.13\end{array}$ $\begin{array}{llll}552.775273 & 25.5547744 \quad 0.06\end{array}$ $\begin{array}{lll}1990.557668 & 90.52906827 & 0.10\end{array}$ $\begin{array}{llll}2259.573764 & 119.4861298 & 0.08\end{array}$ $\begin{array}{llll}2978.686846 & 62.92350623 & 0.14\end{array}$ $\begin{array}{llll}9762.328213 & 10.43913043 & 0.21\end{array}$ $\begin{array}{llll}0.029838032 & 1640.079712 \quad 0.64\end{array}$ $\begin{array}{llll}0.037564057 & 1325.615014 & 0.60 \\ 0.059702748 & 557.4793131 & 0.76\end{array}$ $\begin{array}{llll}0.059702748 & 557.4793131 & 0.76 \\ 0.066442466 & 296.4389789 & 0.79\end{array}$ $\begin{array}{llll}0.066442466 & 296.4389789 & 0.79 \\ 0.097976937 & 260.3315449 & 0.81\end{array}$ $\begin{array}{llll}0.010527403 & 184.8914536 & 0.87\end{array}$ $\begin{array}{llll}0.105102724 & 120.1802032 & 0.87 \\ 0.108126 & 0.87\end{array}$ $\begin{array}{llll}0.119866763 & 249 & 2785087 & 0.71\end{array}$ $\begin{array}{llll}0.124027187 & 257.1526752 & 0.75\end{array}$ $\begin{array}{llll}0.139009756 & 187.4889038 & 0.68\end{array}$ $\begin{array}{llll}0.171839864 & 141.0460562 & 0.87\end{array}$ $\begin{array}{llll}0.177846417 & 206.4155562 & 0.73\end{array}$ $\begin{array}{llll}0.193765491 & 197.7144369 & 0.73\end{array}$ $\begin{array}{llll}0.192947737 & 153.5425296 & 0.67\end{array}$ $\begin{array}{llll}0.233924744 & 118.2955269 & 0.81\end{array}$ $\begin{array}{llll}0.28631775 & 47.7700269 & 0.86 \\ 0.304570651 & 98.080482 & 0.67\end{array}$ $\begin{array}{llll}0.296193118 & 67.55632983 & 1.06\end{array}$ $\begin{array}{llll}0.304648674 & 73.03904648 & 0.78\end{array}$ $\begin{array}{llll}0.338650364 & 83.31511406 & 0.88\end{array}$ $\begin{array}{llll}0.357200413 & 56.43121815 & 0.75\end{array}$ $\begin{array}{lll}0.359125657 & 46.4778513 & 0.87\end{array}$ $\begin{array}{lll}0.368156472 & 99.1018225 & 0.50\end{array}$ $\begin{array}{llll}0.385438746 & 61.99231791 & 0.97\end{array}$ $\begin{array}{llll}0.390159103 & 77.75524584 & 0.78\end{array}$ $\begin{array}{lll}0.40086734 & 56.22333651 & 1.02 \\ 0.409969951 & 76.85126868 & 0.60\end{array}$ $\begin{array}{llll}0.410973201 & 48.25150765 \quad 0.96\end{array}$ $\begin{array}{llll}0.410973201 & 48.25150765 & 0.96 \\ 0.459726911 & 63.43242197 & 0.68\end{array}$ $\begin{array}{llll}0.449931022 & 58.96065523 & 0.81\end{array}$ $\begin{array}{llll}0.454686458 & 53.87983245 & 0.92\end{array}$ $\begin{array}{llll}0.481198281 & 46.48328833 & 0.69\end{array}$ $\begin{array}{llll}0.465284752 & 36.54938538 & 1.13\end{array}$ $\begin{array}{llll}0.4792539 & 55.64803887 & 0.63\end{array}$ $\begin{array}{llll}0.503754916 & 51.18579643 & 0.90\end{array}$ $\begin{array}{llll}0.522116477 & 46.92066691 & 0.75\end{array}$ $\begin{array}{llll}0.522945733 & 53.9331699 & 0.86\end{array}$ $\begin{array}{llll}0.566382706 & 33.81515753 & 0.69 \\ 0.564960467 & 84.40674052 & 0.56\end{array}$ $\begin{array}{llll}0.56675426 & 41.440394 & 0.71\end{array}$ $\begin{array}{llll}0.614773152 & 45.42218898 & 0.93\end{array}$ $\begin{array}{llll}0.630115813 & 45.95630337 & 0.76\end{array}$ $\begin{array}{llll}0.645078606 & 55.77316633 & 0.67\end{array}$ $\begin{array}{llll}0.669534161 & 44.26911979 & 0.72\end{array}$ $\begin{array}{llll}0.652689453 & 44.97979368 & 0.8\end{array}$ $\begin{array}{lll}0.687042244 & 42.86391898 & 0.83\end{array}$ $\begin{array}{llll}0.799333158 & 32.14227561 & 1.05\end{array}$ $\begin{array}{llll}0.802518865 & 33.6579648 & 0.87\end{array}$ $\begin{array}{llll}0.80881984 & 35.50450576 & 0.79\end{array}$ $\begin{array}{llll}0.832623191 & 33.88206453 & 0.67 \\ 0.83967169 & 30.39715049 & 0.79\end{array}$ $\begin{array}{llll}0.858278567 & 25.37281469 & 0.84\end{array}$ $\begin{array}{llll}0.879564905 & 27.55976753 & 0.66\end{array}$ $\begin{array}{llll}0.908990916 & 29.18477209 & 0.80\end{array}$ $\begin{array}{llll}0.909743899 & 27.11835298 & 0.91\end{array}$ $\begin{array}{llll}0.913201909 & 31.5348743 & 0.73\end{array}$ $\begin{array}{llll}1.008959403 & 30.04948942 & 0.69\end{array}$ $\begin{array}{llll}1.129200888 & 26.01157245 & 0.82\end{array}$ $\begin{array}{lll}1.161938465 & 18.66692457 & 0.68 \\ 1.199174628 & 22.65731599 & 0.84\end{array}$ $\begin{array}{lll}1.199174628 & 22.65731599 & 0.84 \\ 1.22484847 & 20.40234083 & 0.98\end{array}$ $\begin{array}{llll}1.22484847 & 20.40234083 & 0.98 \\ 1.252262087 & 20.18019717 & 0.86\end{array}$ $\begin{array}{lll}1.252262087 & 20.18019717 & 0.86 \\ 1.355871654 & 20.43855936 & 0.83\end{array}$ $\begin{array}{llll}1.366768202 & 22.96151803 & 0.68\end{array}$ $\begin{array}{llll}1.438515593 & 20.14901895 & 0.59\end{array}$ $\begin{array}{llll}1.439055379 & 21.73162147 & 0.78\end{array}$ $\begin{array}{llll}1.470993685 & 19.54680203 & 0.85\end{array}$ $\begin{array}{llll}1.525925054 & 18.23480715 & 0.62\end{array}$ $\begin{array}{lll}1.586513249 & 21.81572588 & 0.6\end{array}$ $\begin{array}{lll}1.629557977 & 15.05414325 & 0.94 \\ 1.663062147 & 18.7994035 & 0.60\end{array}$ 


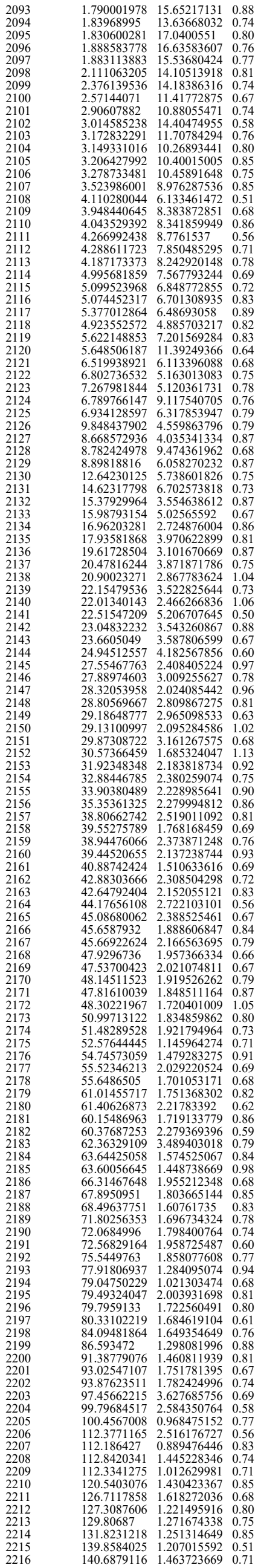




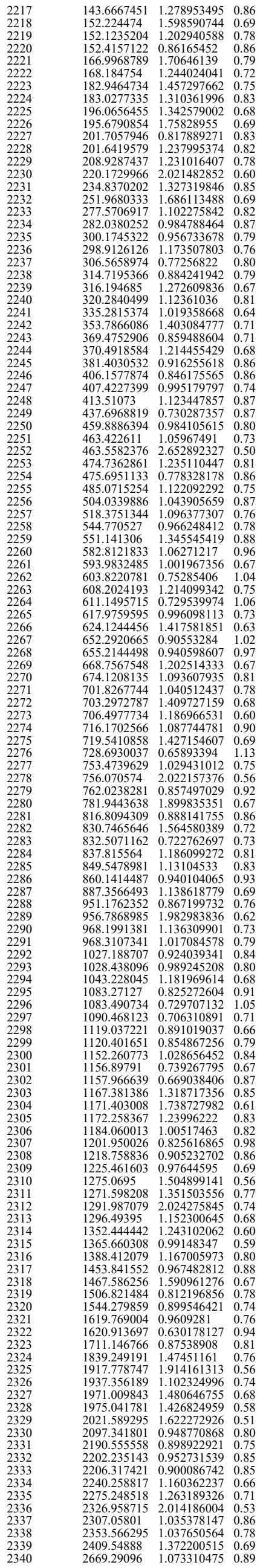



$\begin{array}{llll}2975.641094 & 1.286781295 & 0.75\end{array}$ $\begin{array}{llll}3132.464848 & 0.783647534 & 0.72\end{array}$ $\begin{array}{lll}3387.470381 & 1.03540367 & 0.78\end{array}$ $\begin{array}{llll}4021.486595 & 1.534651548 & 0.5\end{array}$ $\begin{array}{llll}4483.235481 & 0.909829449 & 0.79\end{array}$ $\begin{array}{llll}4902.96334 & 1.743000524 & 0.66\end{array}$ $\begin{array}{llll}5362.275799 & 1.480235654 & 0.55\end{array}$ $\begin{array}{llll}6815.917226 & 1.345386148 & 0.84\end{array}$ $7487.901483 \quad 1.360825015 \quad 0.99$ $\begin{array}{llll}7642.683738 & 1.604031771 & 0.96\end{array}$ $\begin{array}{llll}8002.832699 & 1.929577895 & 0.66\end{array}$ $\begin{array}{llll}8652.448257 & 1.470016726 & 0.57\end{array}$ $\begin{array}{llll}8918.355437 & 1.109554344 & 0.42\end{array}$ $\begin{array}{llll}9285.263272 & 1.466190331 & 0.8\end{array}$ $\begin{array}{llll}9244.509737 & 1.420348998 & 0.68\end{array}$ $\begin{array}{lll}9503.832887 & 1.704895721 & 0.63 \\ 10472.52164 & 1.517083814 & 0.74\end{array}$

Source: (Song et al., 2017)

$\begin{array}{llll}0.002368487 & 9326.293629 & 1.00\end{array}$ $\begin{array}{llll}0.016704064 & 1500.018712 & 1.00\end{array}$ $\begin{array}{llll}0.053477936 & 493.9300699 & 1.00\end{array}$ $\begin{array}{llll}0.131388681 & 193.9612788 & 1.00\end{array}$ $\begin{array}{llll}0.414360509 & 65.81850409 & 1.00\end{array}$ $\begin{array}{llll}0.927449514 & 31.14156087 & 1.00 \\ 1.725255547 & 17.57697384 & 1.00\end{array}$ $\begin{array}{llll}0.035028671 & 763.3216398 & 1.00\end{array}$ $\begin{array}{llll}0.121727749 & 213.3288029 & 1.00\end{array}$ $\begin{array}{llll}0.27399651 & 99.80558056 & 1.00\end{array}$ $\begin{array}{llll}0.883694839 & 32.38277228 & 1.00\end{array}$ $\begin{array}{llll}1.94315632 & 15.8752069 & 1.00\end{array}$ $\begin{array}{llll}3.427449514 & 9.966043521 & 1.00\end{array}$ $\begin{array}{llll}0.009411618 & 2771.461603 & 1.00 \\ 0.073921715 & 359.4043833 & 1.00\end{array}$ $\begin{array}{llll}0.073921715 & 359.4043833 & 1.00\end{array}$ $\begin{array}{lll}0.252430815 & 104.0199111 & 1.00 \\ 0.565445026 & 49.14016965 & 1.00\end{array}$ $\begin{array}{llll}1.749813014 & 17.31840615 \quad 1.00\end{array}$ $3.612066816 \quad 9.633760921 \quad 1.00$ $6.252804787 \quad 6.27895996-1.00$ $\begin{array}{lll}0.013126182 & 1622.449658 & 1.00\end{array}$ $\begin{array}{llll}0.08617624 & 301.13622 & 1.00\end{array}$ $\begin{array}{llll}0.286350519 & 92.04832797 & 1.00\end{array}$ $\begin{array}{llll}0.681420069 & 38.52993023 & 1.00\end{array}$ $\begin{array}{llll}1.926980623 & 16.26099609 & 1.00\end{array}$ $\begin{array}{llll}1.0269870358 & 8.876570397 & 1.00\end{array}$ $\begin{array}{llll}6.799933103 & 6.045591643 & 1.00 \\ 0.186334717 & 143.4151107 & 1.00\end{array}$ $\begin{array}{llll}0.186334717 & 143.4151107 & 1.00 \\ 0.60565917 & 45.81415221 & 1.00\end{array}$ $\begin{array}{llll}0.60565917 & 45.81415221 & 1.00\end{array}$ $\begin{array}{llll}3.771779931 & 9.450472966 & 1.00\end{array}$ $\begin{array}{llll}7.501327797 & 5.663516327 & 1.00\end{array}$ $\begin{array}{llll}12.44561822 & 4.018458933 & 1.00\end{array}$ $\begin{array}{lll}0.04865248 & 550.2181433 & 1.00\end{array}$ $\begin{array}{llll}0.370101269 & 76.06654812 & 1.00\end{array}$ $\begin{array}{llll}1.173223875 & 25.54740828 & 1.00\end{array}$ $\begin{array}{llll}2.471146482 & 13.64984952 & 1.00\end{array}$ $\begin{array}{llll}6.79793564 & 6.087577783 & 1.00 \\ 13.2003737 & 3.826858267 & 1.00\end{array}$ $\begin{array}{lll}13.2003737 & 3.826858267 & 1.00\end{array}$ $\begin{array}{lll}13.75634706 & 2.751445635 & 1.00\end{array}$ $\begin{array}{llll}0.399537778 & 64.09989045 \quad 1.00\end{array}$ $\begin{array}{llll}0.182293333 & 24.70564988 & 1.00\end{array}$ $\begin{array}{llll}2.636586667 & 11.77548682 \quad 1.00\end{array}$ $\begin{array}{llll}6.803626667 & 5.968370864 & 1.00\end{array}$ $\begin{array}{llll}13.24216889 & 3.734519365 & 1.00\end{array}$ $\begin{array}{llll}21.88995556 & 2.669274516 & 1.00\end{array}$ $\begin{array}{lll}0.81056 & 34.50687563 & 1.00\end{array}$ $\begin{array}{lll}0.310564 & 34.50687563 & 1.00 \\ .811377778 & 7.8466084 & 1.00\end{array}$ $\begin{array}{lll}4.811377778 & 7.834784327 & 1.00\end{array}$ $\begin{array}{llll}12.48117333 & 3.92942415 & 1.00\end{array}$ $37.90471111 \quad 1.972420946 \quad 1.00$ $\begin{array}{llll}0.924248889 & 1.97256282 & 1.00\end{array}$ $\begin{array}{llll}0.52864 & 20.2583118 & 1.00\end{array}$ $\begin{array}{lll}4.369226667 \quad 8.369091503 & 1.00\end{array}$ $\begin{array}{llll}8.458595556 & 5.293064151 & 1.00\end{array}$ $\begin{array}{lll}21.42816 & 2.783605548 & 1.00\end{array}$ $\begin{array}{llll}39.41219556 & 1.950439153 & 1.00\end{array}$ $\begin{array}{llll}6.9287111 & 1.494259514 & 1.00\end{array}$ $\begin{array}{llll}0.026292591 & 1.456 .313595 & 0.81\end{array}$ $\begin{array}{llll}0.220857766 & 131.101292 & 0.81 \\ 0.722891597 & 41.30092205 & 0.81\end{array}$ $\begin{array}{llll}1.581267369 & 20.44028561 & 0.81\end{array}$ $\begin{array}{llll}2.804800836 & 12.70130347 & 0.81\end{array}$ $\begin{array}{llll}0.064648842 & 427.9911382 & 0.81\end{array}$ $\begin{array}{lllll}0.492135443 & 59.08496419 & 0.81\end{array}$ $\begin{array}{llll}1.500533648 & 21.4500626 & 0.81\end{array}$ $\begin{array}{llll}3.122322541 & 11.74304492 & 0.81\end{array}$ $\begin{array}{llll}5.290997331 & 7.987138309 & 0.81\end{array}$ $\begin{array}{llll}0.130689645 & 219.6071441 & 0.81\end{array}$ $\begin{array}{llll}0.949007881 & 33.31801867 & 0.81 \\ 2.797377045 & 12.94167738 & 0.81 \\ 5.64084357 & 7.544330364 & 0.81\end{array}$ $\begin{array}{llll}2.797377045 & 12.94167738 & 0.81\end{array}$ $\begin{array}{llll}9.640371074 & 5.416595853 & 0.81\end{array}$ $\begin{array}{llll}0.143384679 & 207.7466168 & 0.81\end{array}$ $\begin{array}{llll}0.14338467565 & 207.7458588 & 0.81\end{array}$ $\begin{array}{llll}3.167208232 & 11.49608544 & 0.81\end{array}$ $\begin{array}{llll}6.389646118 & 6.695234856 & 0.8\end{array}$ $\begin{array}{llll}0.69012436 & 4.671796872 & 0.81\end{array}$ $\begin{array}{llll}0.328545634 & 88.10335534 & 0.81\end{array}$ $\begin{array}{llll}2.179447113 & 16.01699963 & 0.8\end{array}$ $\begin{array}{llll}5.945684669 & 7.263465321 & 0.81\end{array}$ $\begin{array}{llll}11.48635188 & 4.613171385 & 0.81 \\ 18.54264824 & 3.457405687 & 0.81\end{array}$ $\begin{array}{llll}11.48635188 & 4.613171385 & 0.81 \\ 0.6408056 & 48.46002534 & 0.8\end{array}$ $\begin{array}{lllll}4.001317621 & 9.943062463 & 0.81\end{array}$ $\begin{array}{lllll}10.42141086 & 4.947057546 & 0.81\end{array}$ $\begin{array}{lllll}19.75947081 & 3.261862046 & 0.81\end{array}$ $\begin{array}{llll}31.67031214 & 2.479942204 & 0.81\end{array}$ $\begin{array}{llll}0.597698048 & 54.70294269 & 0.81\end{array}$ $\begin{array}{lllll}3.970154914 & 9.918578736 & 0.81\end{array}$ $\begin{array}{llll}10.51108638 & 4.775764673 & 0.8\end{array}$ $\begin{array}{llll}10.51108638 & 4.775764673 & 0.81 \\ 22.6896586 & 3.040643131 & 0.81 \\ 1.34388319 & 2.285933579 & 0.81\end{array}$ $\begin{array}{llll}32.68965861 & 2.285933579 & 0.81 \\ 1.34388319 & 23.97476228 & 0.81\end{array}$ $\begin{array}{lll}1.34388319 & 23.97476228 & 0.8 \\ 7.520346331 & 6.124796402 & 0.81\end{array}$ 


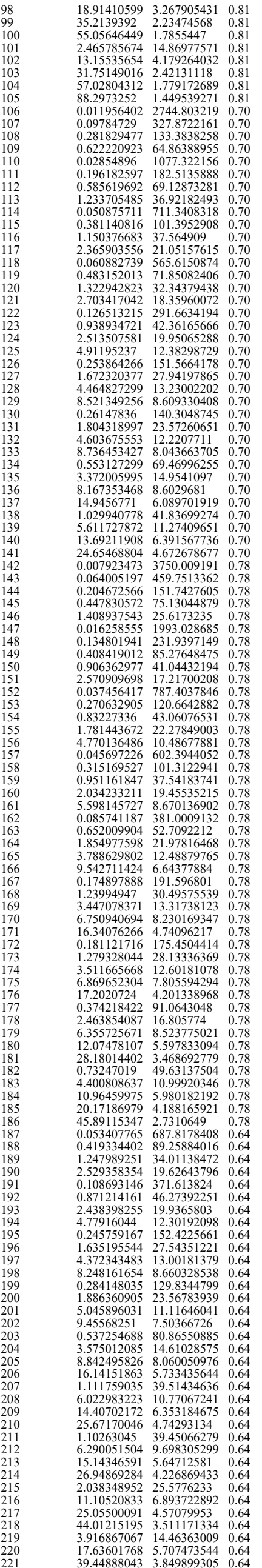




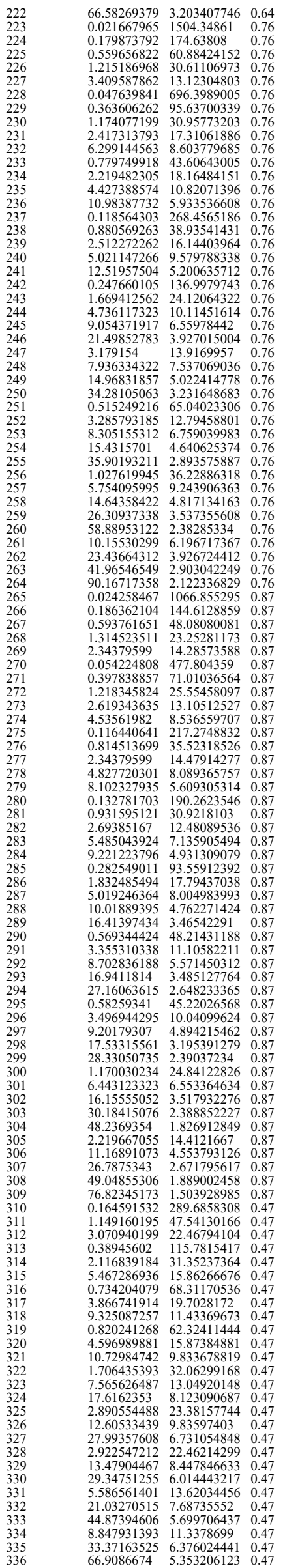

